INEL-94/0174

\title{
DESIGN REPORT ON SCDAP/RELAP5 MODEL IMPROVEMENTS - DEBRIS BED AND MOLTEN POOL BEHAVIOR
}

\author{
C. M. Allison \\ J. L. Rempe \\ S. A. Chavez
}

November 1994

\section{Idaho National Engineering Laboratory Idaho Falls, Idaho 83415}

\author{
Prepared for the \\ Division of Systems Research \\ Office of Nuclear Regulatory Research \\ U. S. Nuclear Regulatory Commission \\ Washington, D. C. 20555 \\ Under DOE Contract No. DE-AC07-94ID13223 \\ DISCLAIMER - FIN No. W6095
}

DISTAIBUTION OF THIS DOCUAENT IS UNLLASTEO

This report was prepared as an account of work sponsored by an agency of the United States Government. Neither the United States Government nor any agency thereof, nor any of their employees, makes any warranty, express or implied, or assumes any legal liability or responsibility for the accuracy, completeness, or usefulness of any information, apparatus, product, or process disclosed, or represents that its use would not infringe privately owned rights. Reference herein to any specific commercial product, process, or service by trade name, trademark, manufacturer, or otherwise does not necessarily constitute or imply its endorsement, recommendation, or favoring by the United States Government or any agency thereof. The views and opinions of authors expressed herein do not necessarily state or reflect those of the United States Government or any agency thereof. 


\section{DISCLAIMER}

Portions of this document may be illegible in electronic image products. Images are produced from the best available original document. 


\section{ABSTRACT}

Improvements for existing debris bed and molten pool models in the SCDAP/RELAP5/MOD3.1 code are described in this report. Model improvements to address (a) debris bed formation, heating, and melting, (b) molten pool formation and growth, and (c) molten pool crust failure are discussed. Relevant data, existing models, proposed modeling changes, and the anticipated impact of the changes are discussed. Recommendations for the assessment of improved models are provided.

FIN NO. W6095-SCDAP/RELAP5 CODE IMPROVEMENT AND ASSESSMENT 


\section{CONTENTS}

ABSTRACT iii

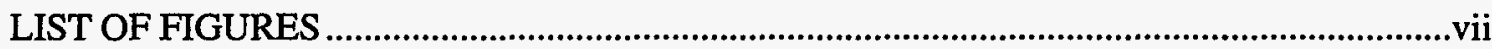

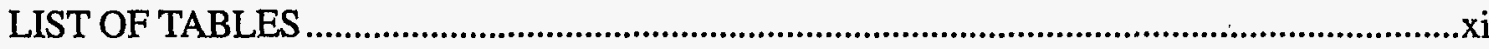

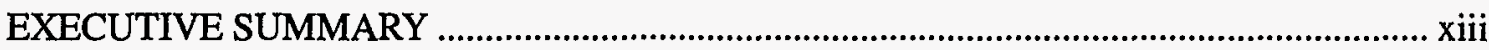

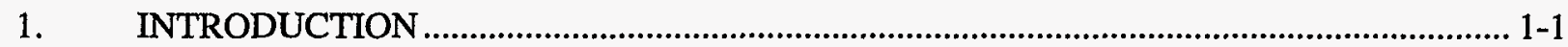

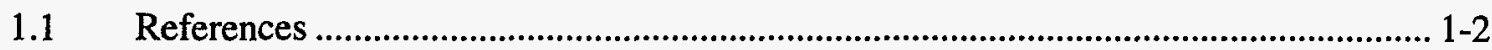

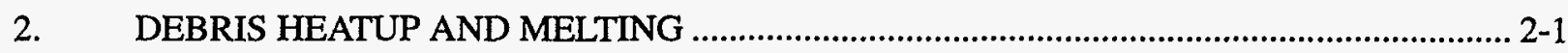

$2.1 \quad$ Review of Pertinent Data...................................................................................... 2-1

2.2 Review of Existing In-Core and Lower Plenum Debris Models................................ 2-23

2.2.1 Transition Criteria for Debris Bed Formation ................................................. 2-23

2.2.2 Debris Bed Characteristics ....................................................................... 2-27

2.2.3 Effective Thermal Conductivity for Lower Plenum Loose Debris ................ 2-27

2.3 Proposed Model Improvements....................................................................... 2-29

2.3.1 Transition Criteria for Debris Bed Formation .............................................. 2-31

2.3.2 Debris Bed Characteristics ......................................................................... 2-32

2.3.3 Effectivethermal conductivity models for lower plenum loose debris.......... 2-34

$2.4 \quad$ Expected Impact of Improved Models ..................................................................... 2-37

2.4.1 Comparison of Effective Thermal Conductivity Models ............................. 2-38

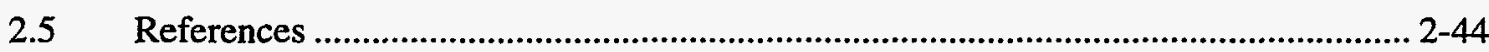

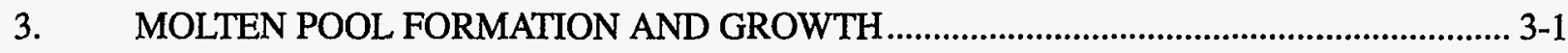

3.1 Molten Pool Formation................................................................................................... 3-1

3.2 Review of Existing In-Core and Lower Plenum Debris Models............................... 3-2

3.2.1 Characteristic Dimensionless Groups .......................................................... 3-2

3.2.2 Typical Ranges for Molten Pool Heat Transfer Parameters............................ 3-3

3.2.3 Data for Modeling Molten Pool Natural Convection...................................... 3-5

3.2.4 Current SCDAP/RELAP5/MOD3.1 Molten Pool Heat Transfer Model ........ 3-5

3.3 Proposed Modifications for Molten Pool Natural

Convection Heat Transfer Model ............................................................................... 3-6

3.3.1 Steady-State Natural Convection ............................................................. 3-6

3.3.2 Transient Natural Convection.......................................................................3-11

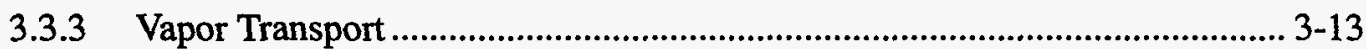

3.3.4 Phenomena Requiring Additional Data and/or Analyses........................... 3-16

3.4 Impact of Proposed Modifications........................................................................... 3-18

3.4.1 Steady-State Heat Transfer Model Modifications ...................................... 3-18

3.4.2 Transient Model Modifications ..................................................................... 3-19

3.4.3 Vapor Transport Model Modifications ...................................................... 3-20 
3.5 Summary and Recommendations....................................................................... 3-21

3.6 References .................................................................................................... 3-23

4. MOLTEN POOL CRUST FAILURE.................................................................................. 4-1

4.1 Review of Crust Data from the TMI-2 Accident.......................................................... 4-1

4.2 Review of Existing Crust Failure Models ................................................................... 4-2

4.3 Proposed Model Improvements................................................................................ 4-3

4.3.1 Bounding Cases for Analyzing Stresses............................................................ 4-3

4.3.2 Assumptions and Input............................................................................. 4-5

4.3.3 Governing Equations for Pressure Stresses....................................................... 4-6

4.4 Impact of Proposed Modifications........................................................................... 4-12

4.4.1 Temperature-Dependent Ultimate Strength...................................................... 4-15

4.4.2 Crust Side-Wall .......................................................................................... 4-15

4.4.3 Crust Top Dome ……………………….......................................................... 4-17

4.5 Summary and Recommendations ....................................................................... 4-19

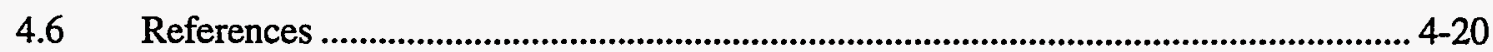

5. RECOMMENDATIONS FOR ASSESSMENT OF IMPROVED MODELS ........................... 5-1

5.1 Debris Transition and Characteristics............................................................................. 5-1

5.2 Debris Heating and Melting ......................................................................................... 5-1

5.2.1 Effective Thermal Conductivity Models for Lower Plenum Loose Debris .... 5-2

5.3 Cohesive Blockage and Molten Pool Formation and Growth....................................... 5-3

5.3.1 Molten Pool Natural Convection...................................................................... 5-3

5.4 Molten Pool Crust Failure ........................................................................................ 5-4

$5.5 \quad$ Remaining Uncertainties ................................................................................. 5-4

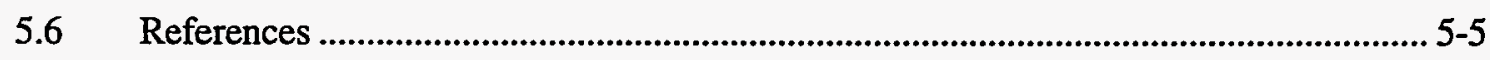

APPENDIX A-REVIEW OF MOLTEN POOL NATURAL CIRCULATION HEAT TRANSFER EXPERIMENTS AND CORRELATIONS..........................................................................A-1

APPENDIX B-COMPARISON OF STRESSES IN SIDE-WALL AND BOTTOM CRUSTS.............. B-1 


\section{LIST OF FIGURES}

Figure 2-1. Hypothesized core damage configuration at 150-160 minutes...........................2-2

Figure 2-2. Hypothesized core damage configuration at 173 minutes..................................2-3

Figure 2-3. SCDAP/RELAP5//MOD3.1 predicted temperatures radially across the core..............................................................................................................

Figure 2-4. Hypothesized core damage configuration (175-180 minutes)...........................2-4

Figure 2-5. Hypothesized core damage configuration (226 minutes). ...................................2-4

Figure 2-6. Artist's rendition of the damage occurring in the CORA-2 experiment............2-5

Figure 2-7. Metallographic cross-sections and external view for the CORA-2

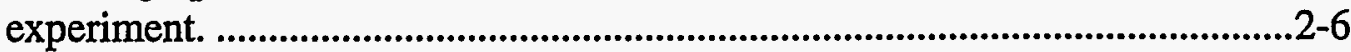

Figure 2-8. Artist's rendition of the damage occurring in the CORA-3 experiment............2-7

Figure 2-9. Metallographic cross-sections and external view for the CORA-3

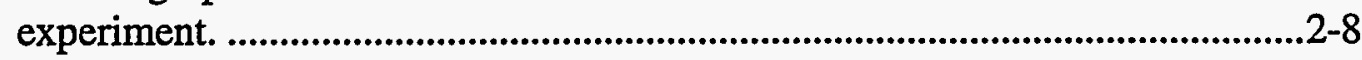

Figure 2-10. Enlargement of the refrozen melt and powdery rubble from ESBU-2A. ..........2-9

Figure 2-11. Enlarged view of the ESBU-2A blocked region from above: two orientations............................................................................................................. $2-9$

Figure 2-12. Sample of lower crust from center of TMI-2 core............................................2-10

Figure 2-13. Ceramic melt temperatures were based on weighted values. ..........................2-11

Figure 2-14. LOFT LP-FP-2 metallographic cross-section from the region where a ceramic blockage occurred (NE corner)............................................................................2-11

Figure 2-15. LOFT LP-FP-2 metallographic cross-section from the region where a ceramic blockage occurred (NW corner). .....................................................................2-12

Figure 2-16. LOFT LP-FP-2 metallographic cross-section from the region where ceramic blockage occurred (SW corner).

Figure 2-17. LOFT LP-FP-2 metallographic cross-section from the region where a ceramic blockage occurred (SE corner).

Figure 2-18. Metallographic cross-sections and external view for the CORA-5 experiment.

Figure 2-19. Close-up metallographic cross-sectional view of the porosity in the Zr-In-ss blockage in the CORA-5 experiment.

Figure 2-20. Close-up metallographic cross-sectional view of the porosity in the upper portion of the $\mathrm{Zr}-\mathrm{B}_{4} \mathrm{C}$-ss blockage in the $\mathrm{DF}-4$ experiment.

Figure 2-21. Close-up metallographic cross-sectional view of the porosity in a middle portion of the $\mathrm{Zr}-\mathrm{B}_{4} \mathrm{C}$-ss blockage in the $\mathrm{DF}-4$ experiment. ................2-16

Figure 2-22. Enlargement of the rubble found above the refrozen melt in the ESBU-2A experiment.

Figure 2-23. Metallographic cross-sections from the PBF SFD 1-4 experiment.................2-17

Figure 2-24. Metallographic cross-sections and external view from the CORA-12 experiment.

Figure 2-25. Closeup metallographic cross-sectional view from the $\mathrm{Zr}$-Ag-ss blockage in the CORA-12 experiment. 
Figure 2-26. Closeup metallographic cross-sectional view of the blockage region in the CORA-12 experiment.

Figure 2-27. Metallographic cross-sections and external view for the CORA-17 experiment.

Figure 2-28. Metallographic cross-sections and external view from the CORA-16 experiment.

Figure 2-29. Transition criteria for intact to rubble debris geometry.

Figure 2-30. Transition criteria for intact to metallic blockage geometry. $2-25$

Figure 2-31. Transition criteria for rubble debris or intact geometries to ceramic melt geometry.

Figure 2-32. Important damage progression stages and melting temperatures....................2-34

Figure 2-33. Effective conductivity predicted by the INEL Imura-Takegoshi and Vortmeyer model.

Figure 2-34. INEL Imura-Takegoshi and Vortmeyer model sensitivity to particle diameter.

Figure 2-35. INEL Imura-Takegoshi and Vortmeyer model sensitivity to debris bed porosity. $2-40$

Figure 2-36. Comparison of various effective conductivity model predictions....................2-41

Figure 2-37. Sensitivity of Nasr, et al. model predictions to particle diameter. ....................2-42

Figure 2-38. Sensitivity of Nasr, et al. model predictions to debris bed porosity.................2-42

Figure 2-39. Sensitivity of Nasr, et al. model predictions to particle composition. .............2-43

Figure 3-1. Molten pool formation in the reactor core......................................................3-1

Figure 3-2. Heat transfer mechanisms associated with molten pools formation...................3-2

Figure 3-3. Comparison of high Rayleigh number correlations for upward heat transfer (to side walls).

Figure 3-4. Comparison of high Rayleigh number correlations for horizontal heat transfer (to side walls).

Figure 3-5. Comparison of high Rayleigh number correlations for predicting downward heat transfer.

Figure 3-6. Comparison of local heat transfer results. $3-10$

Figure 3-7. Comparison of transient and steady-state correlations as a function of transient Rayleigh number.

Figure 3-8. Ratios of heat transfer coefficients as a function of pool void fraction. ..........3-16

Figure 3-9. Comparison of heat transfer coefficients predicted when geometry effects are considered.

Figure 3-10. Comparison of the transient and steady-state Nusselt numbers for the Section 3.2.2 SCDAP/RELAP5 molten pool.

Figure 3-11. Impact of boiling on heat transfer from a molten pool....................................3-21

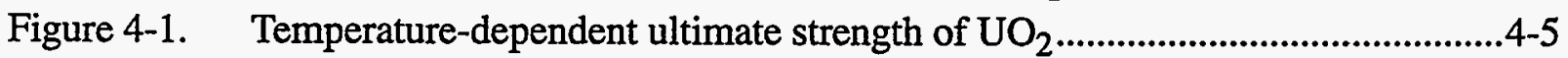

Figure 4-2. Schematic of top crust dome geometry.......................................................4-8

Figure 4-3. Comparison of approximate solution and solution with end effects for side-wall hoop stresses in a closed cylinder. 
Figure 4-4. Schematic of TMI-2 endstate.

Figure 4-5. Side-wall failure map for a open crucible with molten interior and linear through-wall temperature distribution.

Figure 4-6. Side-wall failure map for a closed crucible with molten interior and linear through-wall temperature distribution. ................................................................4-17

Figure 4-7. TMI-2 accident pressure history. .......................................................................4-18

Figure 4-8. Dome top failure map for a closed crucible with molten interior and linear through-wall temperature distribution, assuming $1710 \mathrm{~K}$ outer wall temperature.

Figure 4-9. Comparison of maximum stress solutions for top crust, modeled with dome geometry and modeled with Engelstad and Feng plate geometry. 

. 


\section{LIST OF TABLES}

Table 2-1. Comparison of estimates for radiation exchange factor. $2-28$

Table 2-2. Typical parameters and equations for estimating form and radiation effects factors. $2-36$

Table 2-3. Typical particle characteristics.......................................................................... 2-36

Table 3-1. Ranges for variables used to estimate pool heat transfer parameters. ................ 3-4

Table 4-1. Bounding cases for structural analysis in the top crust..................................... 4-4

Table 4-2. Bounding cases for structural analysis in the side crust. ..................................... 4-4

Table 4-3. Input for analysis of pressure stresses for the top crust. ..................................... 4-6

Table 4-4. Input for analysis of pressure stresses for the side crust.................................... 4-6

Table 5-1. Data sources for assessing porous debris bed effective conductivity model.... 5-2 


\section{EXECUTIVE SUMMARY}

The SCDAP/RELAP5 computer code is designed to describe the overall reactor coolant system (RCS) thermal-hydraulic response, core damage progression, and, in combination with VICTORIA, fission product release and transport during severe accidents. The code is being developed at the Idaho National Engineering Laboratory (INEL) under the primary sponsorship of the Office of Nuclear Regulatory Research of the U.S. Nuclear Regulatory Commission (NRC).

The objective of this design report is to describe proposed improvements in existing debris bed and molten pool models. Model improvements to address (a) debris bed formation, heating, and melting, (b) molten pool formation and growth, and (c) molten pool crust failure are discussed. Relevant data, existing models, proposed modeling changes, and the anticipated impact of the changes are discussed. Recommendations for the assessment of improved models are provided.

The SCDAP/RELAP5 peer review committee noted that the physical understanding of important phenomena associated with debris and molten behavior was limited, severely restricting the development of improved models. However, additional data have become available, and in some cases, new correlations have been developed. This new information, in addition to earlier data, has been used in a number of cases to develop a complete model. In other cases, the assessment of the data by the experimentalists is still incomplete so it is only possible to develop the basic framework for a new model. Specific parameters for that new model can then be refined as the assessment of the data is completed. In most cases, these parameters would be incorporated during the developmental assessment of the new model.

The model improvements recommended in this report address specific modeling deficiencies identified by the peer review committee, including treatment of (a) the effects of conduction and radiation heat transfer in porous debris beds, (b) the melting of porous and non-porous debris, (c) the transition between intact, debris bed, and molten pool geometries, (d) the transient start-up of natural circulation heat transfer in molten pools, and (e) extension of existing cohesive debris bed models to account for the inclusion of closed and open porosity. Important modeling deficiencies that have been identified through analysis of the later stages of TMI-2, full plant calculations, and review of recent experiments will also be addressed. These deficiencies include (a) the abrupt transitions from intact geometries to different types of debris beds or molten pools, (b) the inability to accurately predict the failure and subsequent drainage of the molten pool when the molten pool is surrounded by water, and (c) an inaccurate portrayal of rubble bed particle sizes and porosities.

Specific model recommendations in debris bed formation, heating, and melting; molten pool formation and growth; and molten pool crust are summarized below.

\section{Debris bed formation, heating, and melting}

Fundamental changes in the debris formation and characteristics models are proposed so that they can better represent the observed behavior from existing bundle meltdown experiments. Good examples include the (a) gradual transition from an intact geometry to ceramic cohesive debris bed geometry, (b) the systematic variation in rubble debris porosity and particle sizes depending on peak temperatures and cooling rates, and (c) large increases in closed porosity of metallic blockages in the presence of control and 
structural materials. Possible changes in effective thermal conductivity correlations for rubble debris beds are proposed based on initial assessments of more recently-developed correlations. The incorporation of a thermal equilibrium model to treat the relocation of molten material within a loose rubble is proposed for the detailed two-dimensional COUPLE lower plenum debris model.

The most noticeable impact of the new models will be a much more accurate representation of the formation and behavior of debris beds relative to the currently available data base. Cohesive debris beds will form more gradually over a temperature range more consistent with (a) the range of melting temperatures of different mixture of materials and (b) the rod-to-rod variations in power and cooling conditions in a representative assembly. Rubble debris beds will form over a range of peak temperatures and cooling rates as shown in both the quenched and slow-cooled experiments. More importantly, rubble debris beds will not always be assumed to be spatially uniform. The proposed changes in debris bed characteristics models will also have a significant impact on the subsequent heating and melting of debris beds. The incorporation of more detailed thermal-hydraulic models will be much more influenced by the local rubble bed porosities and particle sizes than the previous models during the dry out and quenching of debris beds. The convective heat transfer and flow patterns in single phase steam will also be impacted since debris-bed-specific friction factors and heat transfer coefficients will be used rather than existing RELAP5 bundle correlations. The effective heat transfer within debris beds will also depend strongly on the porosity and effective particle size.

\section{Molten pool formation and growth}

The following modifications to the SCDAP/RELAP5 molten pool heat transfer models are proposed.

- A transient form of heat transfer correlations obtained from the Kymäläinen, et al. COPO tests should be incorporated. It is recommended that these correlations be used because they were obtained from higher Rayleigh number tests and they are consistent with data obtained from several other two- and three-dimensional investigations. Switching to these correlations would include the use of a separate, horizontal, heat transfer coefficient and allow non-hemispherical geometries to be modeled. Cylindrical and hemispherical pool shapes and aspect ratios will be included.

- The effects of vapor transport on convection heat transfer should be included. Although additional data are needed to validate the proposed modifications, it is suggested that horizontal heat transfer be modeled using correlations proposed by Greene, et al. and that upward and downward heat transfer be modeled using the Ginsberg and Greene correlation. Use of this method requires selecting a boiling regime based on the average void fraction of the melt and calculating a bubble terminal vapor velocity. It is recommended that the pool void fraction be based on the quantity of materials that vaporize as debris is subsumed into the growing pool.

The implementation of these changes will result in a number of positive changes in the code. The recommended correlations better represent the higher Rayleigh numbers expected in plant calculations. The Kymäläinen, et al. COPO correlations are consistent with many of the correlations recommended from previous experiments. In addition, these correlations would allow the code to model semicircular segment and torispherical geometries, rather than mapping these geometries onto a hemispherical geometry. Results 
indicate that heat transfer coefficients vary by as much as $20 \%$, when the proper geometry is considered. In addition, the incorporation of a transient form of these correlations can reduce the heat transfer by more than a factor of two during the initial phases molten pool heat up, while the inclusion of vapor transport mechanisms could increase the heat transfer by several orders of magnitude.

\section{Molten pool crust failure}

A new model that better represents both the thermal and mechanical aspects of crust failure, and the implications of the implementation of the new model is proposed. Although this new model clearly represents more of the critical processes associated with crust failure, it must be noted that the precise timing and location of the failure will still have relatively large uncertainties. To account for these uncertainties a bounding approach, which allows the user to bound the mass or energy of the melt relocating after crust failure, is recommended. The upper and lower bounds consider uncertainties in permeability, high temperature mechanical properties, crust thickness and stress concentrations from flaws. Effects of the uncertainties in crust failure will be established during the developmental assessment process and grouped in a consistent manner so that a reasonable upper and lower bound on the crust failure timing and location can be established easily by the code user. This bounding approach is consistent with the bounding approach used in the current slumping models. As a result, the user should be able to quickly establish an upper and lower bound on the melt mass and energy at lower head failure caused by uncertainties in crust failure and melt slumping processes by selecting a single option in the code.

The proposed model changes will have the most significant impact on plant calculations where, like TMI-2, the core is partially or totally reflooded during molten pool formation and crust failure is likely. For these conditions, the current thermally-based models cannot predict the location nor the timing of crust failure without user intervention. For the TMI-2 calculations, this either resulted in no failure at all because the outer assemblies remained relatively cool following the reflooding of the core or bottom failure of the molten pool for an extreme core uncovery scenario. The new models will be able to account for the likely changes in crust failure thicknesses due to (a) rapid pressure fluctuations, which increases the loading on the crust and (b) the height of the molten pool. In general, the combination of structural and thermally based models should be better able to predict both location and timing of failure because the strength of the crust and loading applied to the crust are considered.

\section{Assessment of the new models}

The assessment of the proposed new models can utilize a combination of detailed code-to-data comparisons, full plant calculations, TMI-2 analyses, and comparisons with more detailed models. The code-to-data comparisons can be used to assess the debris bed transition and characteristics models, effective thermal conductivity models, and, to a more limited extent, molten pool natural circulation models. Full plant studies can be used to evaluate the overall integration of the models to ensure that "physically unrealistic" behavior and nodalization sensitivities have been significantly reduced over the current version of the code. These calculations can also be used to evaluate the coupled effects of debris formation and flow diversion. The TMI-2 analyses can be used to assess the formation of debris beds, molten pool formation and growth, and molten pool crust failure when the debris beds and molten pool are covered by water. Comparisons with more mechanistic models can be used to assess the transition criteria, metallic and ceramic melt formation, debris heating and initial melting, and molten pool formation. 


\section{INTRODUCTION}

The SCDAP/RELAP5 computer code is designed to describe the overall reactor coolant system (RCS) thermal-hydraulic response, core damage progression, and, in combination with VICTORIA, fission product release and transport during severe accidents. The code is being developed at the Idaho National Engineering Laboratory (INEL) under the primary sponsorship of the Office of Nuclear Regulatory Research of the U. S. Nuclear Regulatory Commission (NRC). The development of the current version of the code, SCDAP/RELAP5/MOD3.1, was started in the spring of $1992 .^{1-1}$ This version of the code was developed in parallel with a formal peer review of SCDAP/RELAP5/MOD3 ${ }^{1-2}$ and contains a number of model and code improvements recommended by the code users and the peer review committee.

Although many of the peer review comments were addressed in MOD3.1, there were a number that could not be incorporated prior to the release of MOD3.1 for general applications. However, in parallel with the release of MOD3.1, a general plan was developed ${ }^{1-3}$ that described a phased approach to address the remainder of the peer review committee concerns. After some iterations on the plan, the following general approach was started. First, the code and modeling improvements undertaken by the code developers would focus on the higher priority models associated with the later stages of an accident. Although most of these models were generally applicable to PWR and BWR designs, design-specific models developed at the INEL would emphasize PWR behavior. Oak Ridge National Laboratory (ORNL) code developers would focus on the higher priority BWR-specific models. Second, the model improvements for the early stages of the accident would be undertaken by Cooperative Severe Accident Research Program (CSARP) member countries with modeling development and assessment guidelines developed by the INEL. In all cases, prior to the incorporation of new or improved models, a design report describing these models would be prepared for NRC review and approval. This design report is one of a series of design reports being prepared to discuss different model and code improvements.

The objective of this design report is to describe proposed improvements in existing debris bed and molten pool models. Model improvements to address (a) debris bed formation, heating, and melting, (b) molten pool formation and growth, and (c) molten pool crust failure are discussed. In each section, relevant data, existing models, proposed modeling changes, and the anticipated impact of the changes are discussed. Recommendations for the assessment of improved models are provided in the final section.

At the time of the peer review, the review committee noted that the physical understanding of important phenomena associated with debris and molten behavior was limited, severely restricting the development of improved models. However, since that time, additional data have become available, and in some cases, new correlations have been developed. This new information, in addition to earlier data, has been used in a number of cases to develop a complete model. Models for effective thermal conductivity in porous debris beds and transient natural circulation in molten pools are good examples where sufficient data or correlations exist to develop final models. In other cases, the assessment of the data by the experimentalists is still incomplete so it is only possible to develop the basic framework for a new model. Specific parameters for that new model can then be refined as the assessment of the data is completed. In most cases, these parameters would be incorporated during the developmental assessment of the new model. Examples in this category include the formation of (a) rubble debris beds as a result of reflood, and (b) porous cohesive debris beds as the result of the melting of mixtures of fuel rod, control rod/blade, and structural materials. In these examples, development of a model that can take into account a range of particle sizes, porosities, and compositions observed in the experiments is possible. However, final particle 
size distributions or debris bed porosities will be established as the assessment of existing experiments or ongoing experiments are completed.

Specific modeling deficiencies identified by the peer review committee that will be resolved by the proposed modeling improvements include the following. More recent effective thermal conductivity correlations that better address the effects of conduction and radiation heat transfer in porous debris beds will be incorporated. The melting of porous and non-porous debris will be better represented through improvements in models to treat the relocation of melt within porous debris and convective and radiative boundary conditions on the exterior of debris beds. Incorporation of improved models for fuel rod fragmentation and the transition between intact, debris bed, and molten pool geometries will reduce the TMI-2 bias in existing models. Replacement of existing steady state molten pool correlations with transient correlations will better represent the transient, startup of natural circulation heat transfer in molten pools. The addition of thermal and structural models for molten pool crust failure will greatly reduce the parametric nature of molten pool crust failure and subsequent drainage of the molten pool. Extension of existing cohesive debris bed models to account for the inclusion of closed and open porosity will allow for the incorporation of experimentally derived porosity correlations. Extension of the existing rubble debris models to allow for the incorporation of porosity and particle size correlations will eliminate the reliance on user input or TMI-2 biases in particle bed sizes and porosities.

Important modeling deficiencies that have been identified through analysis of the later stages of TMI-2, full plant calculations, and review of recent experiments will also be addressed. For example, analysis of the later stages of the TMI-2 accident and the LOFT LP-FP-2 experiment indicated that the transitions from intact geometries to different types of debris beds or molten pools were too abrupt given the typical size of representative flow channels in the core. As a consequence, under some conditions, it was predicted that diversion of flow from one flow channel to another following a transition, resulted in the formation and accelerated heatup of isolated regions of debris beds surrounded by intact assemblies even though such behavior was not observed. Analysis of the TMI-2 accident also indicated that existing parametric models for molten pool crust failure cannot accurately predict the failure and subsequent drainage of the molten pool when the molten pool is surrounded by water rather than superheated steam. Review of data for cohesive debris beds indicated that the refrozen melts can contain a high degree of closed porosity under some conditions. However existing models do not account for closed porosity in determining the properties or overall volume of the blockages formed by refreezing material.

It should be noted that this report does not address all of the deficiencies associated with debris or molten pool behavior that have been noted by the peer review or observed during the assessment or application of the code during the later stages of an accident. Because of the unavailability of data sufficient to develop best estimate, first order models, the following modeling deficiencies are not addressed: (a) interactions of the material slumping from a molten pool in the core with lower structures in the vessel or water in the vessel, (b) the cracking and cooling of cohesive masses of frozen material associated with quench or reflood, and (c) debris bed material properties including density, specific heat, and other thermal properties of mixtures of previously molten material. In these cases, existing bounding or parametric models will be maintained until additional data or information becomes available.

\subsection{References}

1-1. C. M. Allison, et al., SCDAP/RELAP5/MOD3.1 Code Manual, Volumes I-V, NUREG/CR-6150, EGG-2720, October 1993. 
1-2. M. L. Corradini, et al., SCDAP/RELAP5 Independent Peer Review, LA-12481, January 1993.

1-3. Letter C. M. Allison to Y-S. Chen, CMA-72-93, Model Development and Assessment Priorities for FY-1994 and FY-1995, December 6, 1993. 


\section{DEBRIS HEATUP AND MELTING}

As discussed in this section, existing models do not account for the observed wide diversity in the nature of the debris beds. To address this, fundamental changes in the debris formation and characteristics models are proposed so that they can better represent the observed behavior. However, as described in this section, the development of correlations using the existing data base is not yet complete, so the models will be developed in such a fashion that scoping correlations can be incorporated based on the limited review contained in this document and then refined as the experimentalists and analysts develop more general correlations. Debris heating and melting has been observed in a wide variety of experiments, as well as the initial stages of the TMI- 2 accident. As summarized in this section, the experiments clearly show the transition from an intact geometry to a geometry composed of zones of loose debris, highly damaged structures, and cohesive debris beds. In some cases, there is strong evidence of fuel desintering, resulting in the formation of a loose debris bed of a fine powder and very little porosity. In other cases, the fuel remained relatively intact with larger fragments of fuel contained within remnants of oxidized Zircaloy cladding even though the bundle was reflooded with water. In some cases, the cohesive debris beds, formed as a consequence of the refreezing of molten or liquefied assembly materials, contained no apparent open or closed porosity. Cooling and oxidation of the debris occurred only on the exterior or within isolated tortuous flow holes. In other cases, the cohesive debris beds were highly porous with an appearance of a foamy-like structure. That porosity appeared to be closed with very little evidence of oxidation within the porosity. This behavior was also observed in the TMI- 2 accident although the scale was much larger.

The improved models for debris bed formation, with experimentally derived correlations for debris bed particle sizes, porosities, and other bed characteristics are used as the basis for other modeling improvements associated with the heating and melting of these debris beds described in this section. This section ends with a discussion of the anticipated impact of the model improvements.

\subsection{Review of Pertinent Data}

The TMI-2 accident provides the most information on the type of debris that can be formed during the transition from the early to later stages of a severe accident. ${ }^{2-1}$ As shown in Figure 2-1, the first type of debris that was formed occurred between 150-160 minutes into the accident when the water level was near the bottom of the core. Two types of debris regions were forming at this time, a solidified crust (composed primarily of metallic materials from the liquefaction of fuel rod cladding, spacer grids, and control rods) and fuel rod remnants. By 173 minutes (Figure 2-2), the solidified crust at the bottom had grown radially as additional assemblies reached temperatures above $1700 \mathrm{~K}$ and axially as more fuel rod material had relocated. As indicated on Figure 2-3, SCDAP/RELAP5/MOD3.1 calculations ${ }^{2-2}$ indicate that nearly all of the upper portions of the core had reached temperatures above $2000 \mathrm{~K}$ with many of the centermost regions exceeding $2870 \mathrm{~K}$ where a $\mathrm{UO}_{2}-\mathrm{ZrO}_{2}$ eutectic is formed. During and following the B-pump transient at 174 minutes, the debris beds formed earlier may have continued to grow. In addition, two debris types were hypothesized as shown in Figure 2-4, an upper debris bed of fragmented fuel rods and an upper crust of frozen $\mathrm{UO}_{2}-\mathrm{ZrO}_{2}$. It is also hypothesized that a small voided region formed at the top of the core due to the consolidation of the molten material in the molten pool in the centermost region of the core. Later in the accident, between 224-226 minutes, this voided region increased in size, as molten material relocated from the molten pool into the lower plenum (Figure 2-5). In addition, a debris bed was formed in the lower plenum. Even though it was likely composed of primarily $(\mathrm{U}, \mathrm{Zr}) \mathrm{O}_{2}$, the form of the debris bed is 
somewhat different than that in the core. In the core, the molten and frozen $(\mathrm{U}, \mathrm{Zr}) \mathrm{O}_{2}$ was forming around remnants of fuel rods, while in the lower plenum, this material was surrounding structural materials. With the possible exception of the debris in the lower plenum, the type of debris beds or debris regions formed in TMI-2 are consistent with those formed in nearly all of the early phase experiments conducted to date. However, the different types of debris beds that form depend upon the type of materials present, peak temperatures, initial heating rates, and cooling rates. Examples of the different type of debris beds formed during representative experiments are discussed in the remainder of this section.

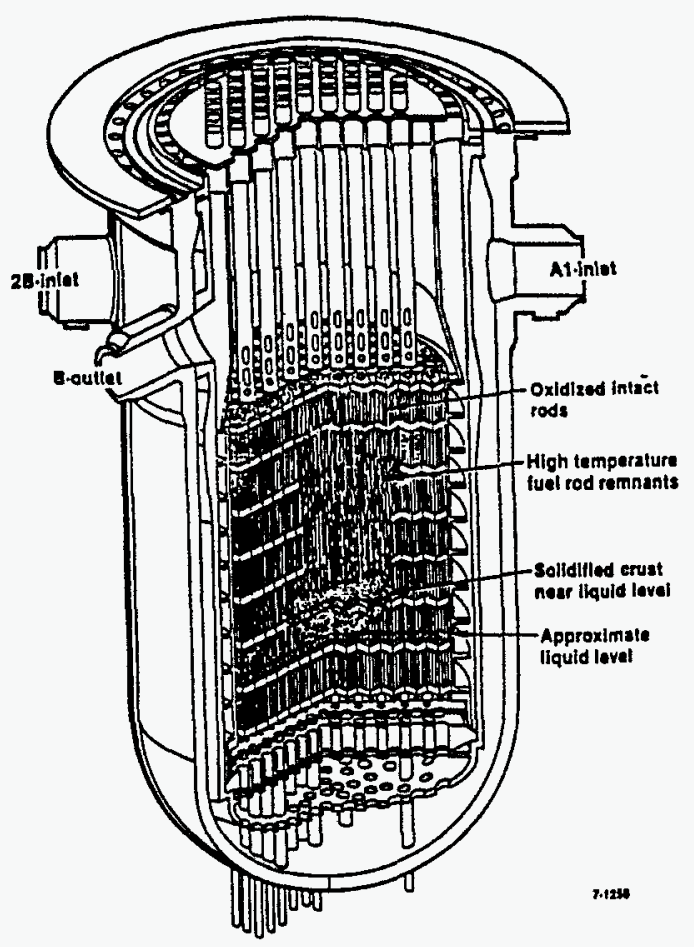

Figure 2-1. Hypothesized core damage configuration at 150-160 minutes. 


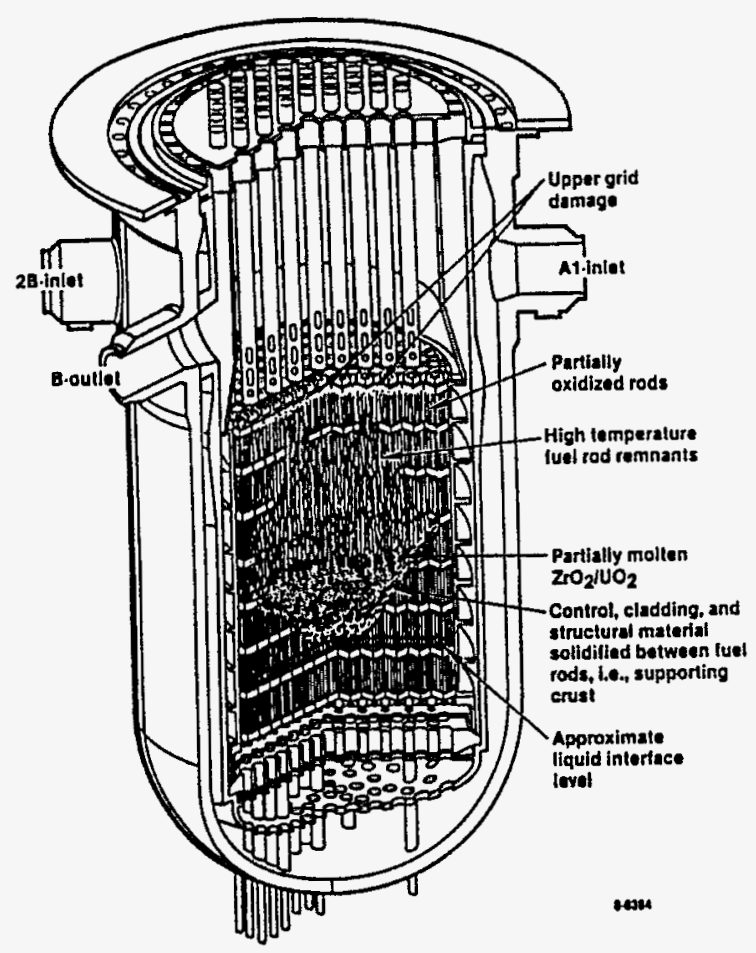

Figure 2-2. Hypothesized core damage configuration at 173 minutes.

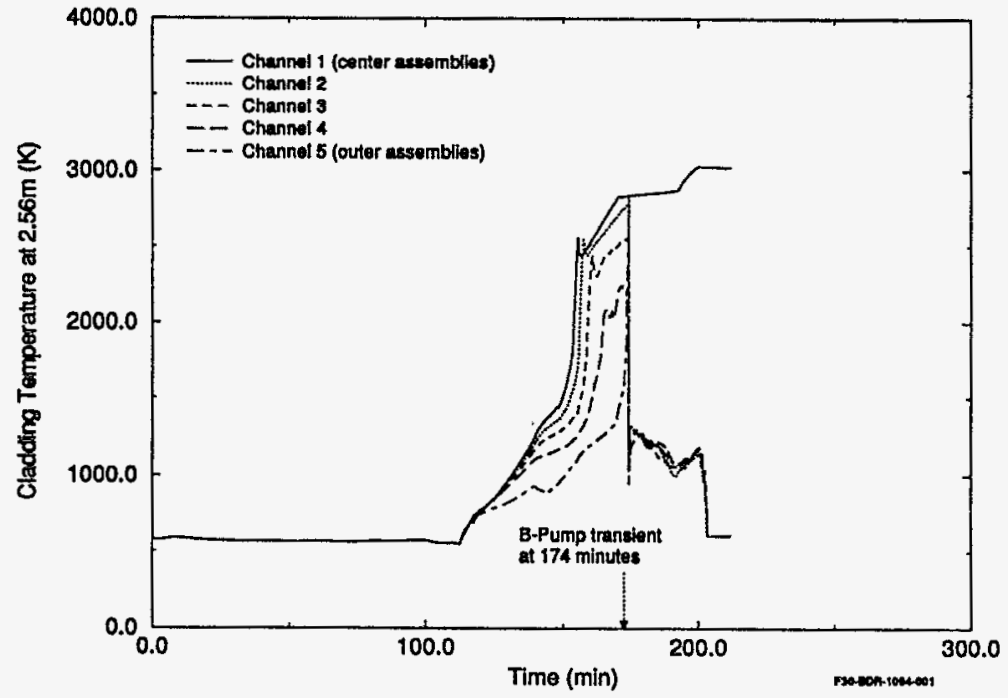

Figure 2-3. SCDAP/RELAP5//MOD3.1 predicted temperatures radially across the core. 


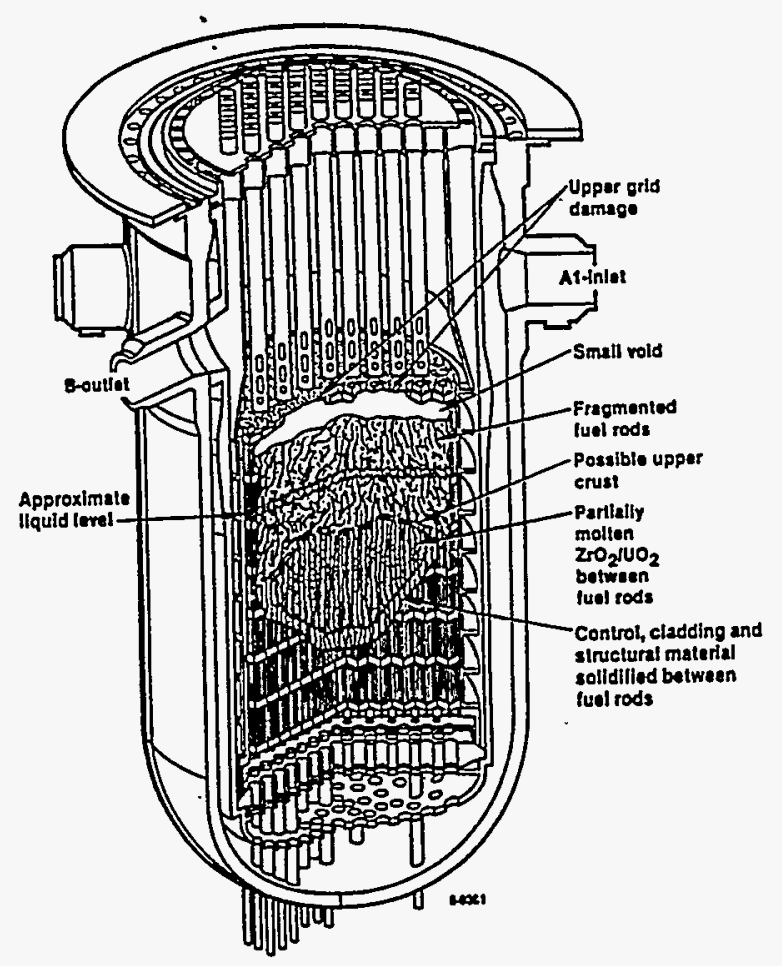

Figure 2-4. Hypothesized core damage configuration (175-180 minutes).

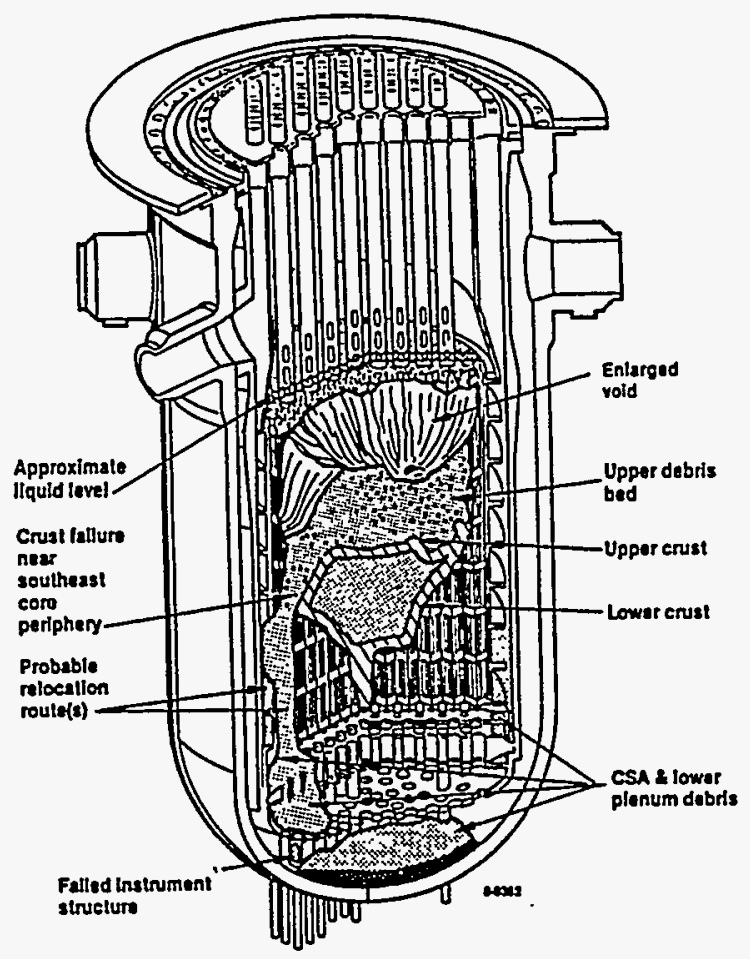

Figure 2-5. Hypothesized core damage configuration (226 minutes). 
For experiments where peak bundle temperatures were limited to $2200 \mathrm{~K}$, the formation of cohesive blockages is still incomplete as shown in the example of the CORA-2 experiment. ${ }^{2-3,2-4}$ As shown in Figure 2-6, an artist's rendition of the damaged bundle, four types of debris beds were observed in the CORA-2 experiment - (1) a lower region composed of loose, fine rubble collecting upon the lower grid spacer, (2) a zone of relocated solidified melt, (3) another zone of loose rubble with an axially varying concentration of rubble surrounding relatively intact fuel rod stubs, and (4) a voided region formed by the collapse of the fuel rods. The metallographic cross sections are shown in Figure 2-7. This experiment was limited to a peak temperature slightly above the melting point of Zircaloy (2150-2250 K), had an initial heating rate of $1 \mathrm{~K} / \mathrm{s}$, and was slowly cooled. The bundle had three grid spacers, a lower and upper grid spacer of Zircaloy and a middle Inconel grid spacer. The bundle was composed of $\mathrm{UO}_{2}$ fuel rods and fuel rod simulators.

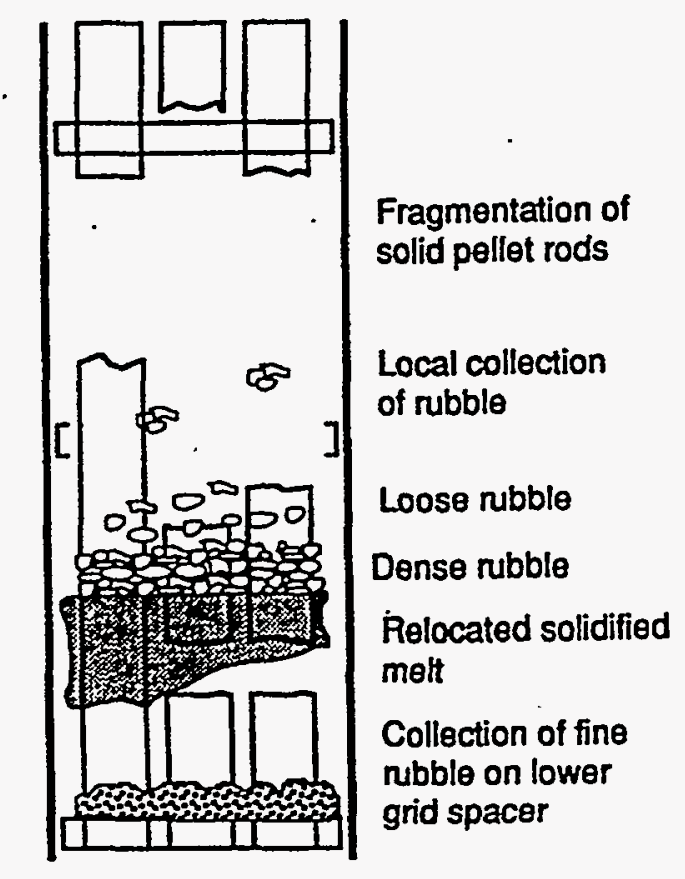

Figure 2-6. Artist's rendition of the damage occurring in the CORA-2 experiment. 


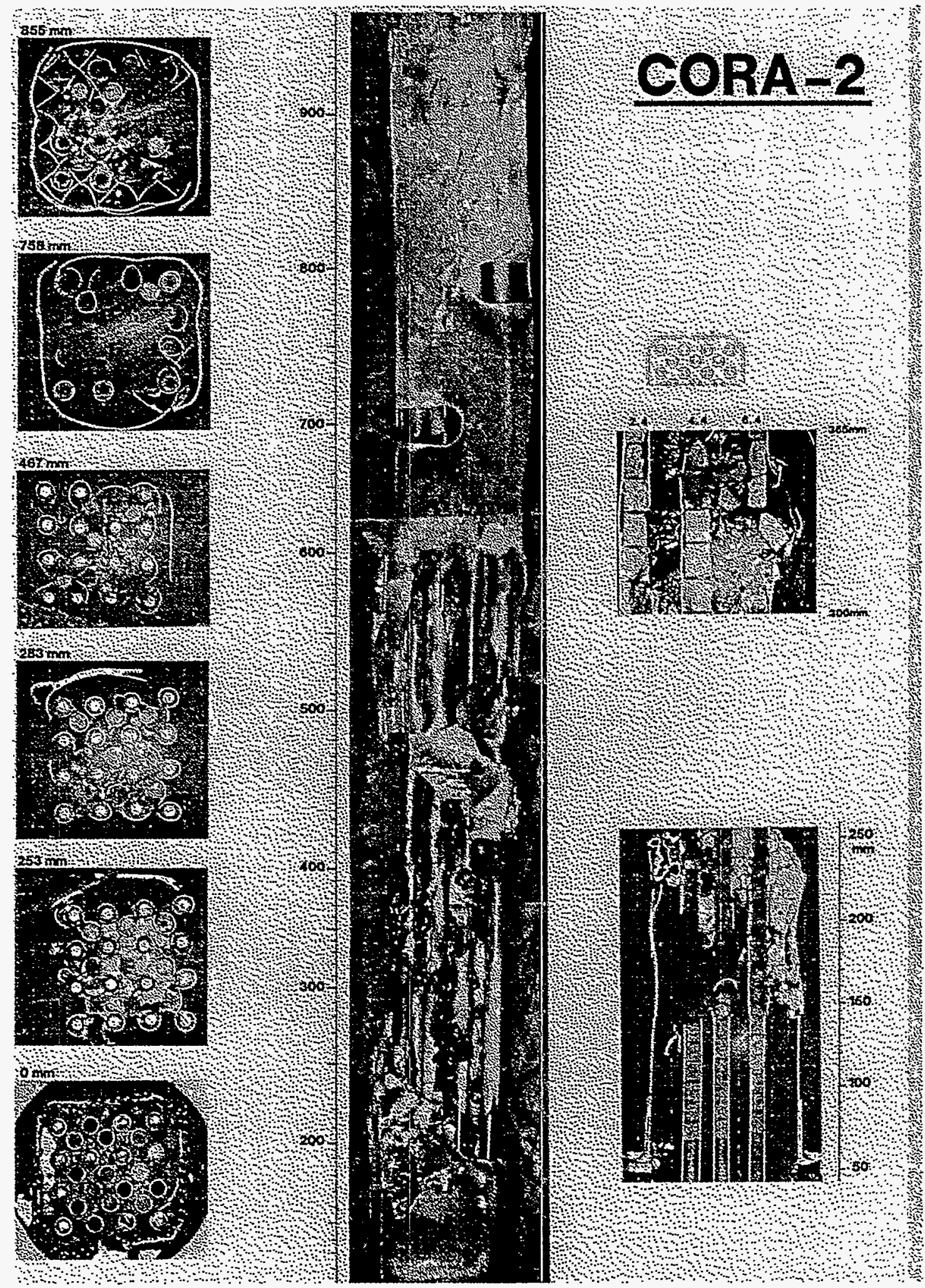

Figure 2-7. Metallographic cross-sections and external view for the CORA-2 experiment. 
The blockage formed at the $253 \mathrm{~mm}$ elevation was composed of Zircaloy and Inconel with small amounts of $\mathrm{UO}_{2}$. Instead of being porous, this blockage was heavily cracked. There is some evidence of oxidation on the outer surface of the blockage. The loose debris was apparently formed during cooldown from the collapse of two regions of fuel. The fine rubble on the lower spacer grid apparently came from the collapse of fuel pellets immediately below the cohesive blockage at the $130-200 \mathrm{~mm}$ elevation. The loose debris sitting on top of the blockage came from the collapse of the fuel columns in the upper half of the bundle. In both cases the fragmented fuel and cladding shards came from regions where the cladding had deformed and opened up. This flowering of the cladding occurred over much of the bundle from $150 \mathrm{~mm}$ and above even though the fuel rods were not prepressurized. They were sealed, however, so the internal pressure increased until the cladding ruptured as the rods heated up.

As the peak bundle temperatures are increased to $2400 \mathrm{~K}$ and above, the cohesive blockage becomes more complete. As shown in Figures 2-8 and 2-9, in the CORA-3 experiment, which was nearly identical to CORA-2, but with a peak temperature between $2600-2700 \mathrm{~K}$, a cohesive blockage is formed on top of the lower grid spacer. The blockage still has isolated flow passages with evidence of oxidation in the flow channels. As was the case for CORA-2, there is little evidence of closed porosity in the frozen melt although it is heavily cracked. In a similar experiment, ESBU-2A, ${ }^{2-5}$ with peak temperatures in excess of $2400 \mathrm{~K}$, the blockages are very similar. The blockage formed in the lower portion of the ESBU-2A bundle was nearly identical to that of the CORA-3 experiment as shown in Figures 2-10 and 2-11. These photographs also show very clearly the difference in the upper and lower surface of the cohesive melt. The bottom of the blockage (Figure 2-10) indicates that the initial blockage formed as an accumulation of rivulets that did not wet the surface of the cladding very well. The upper surface (Figure 2-11) shows that the later accumulation of melt wet the rods well. The blockages occurring in CORA-3 and ESBU-2A also look very much like the lower crust samples taken from TMI-2 (Figure 2-12). The TMI-2 lower crust consisted of $\mathrm{UO}_{2}$ pellets and small, solid $\mathrm{UO}_{2}$ particles surrounded by previously molten structural and control materials. The composition of the metallic material was principally $\mathrm{Zr}, \mathrm{U}, \mathrm{Fe}$ and significant amounts of $\mathrm{Ag}$ and In.

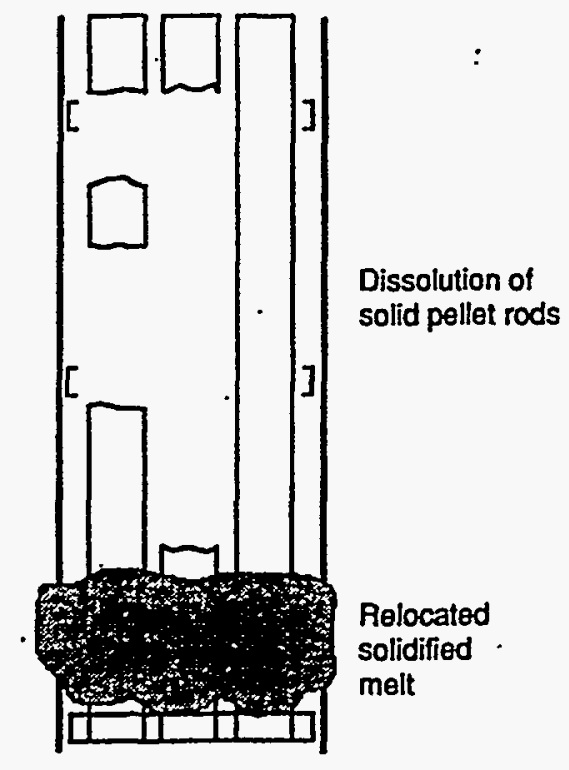

Figure 2-8. Artist's rendition of the damage occurring in the CORA-3 experiment. 


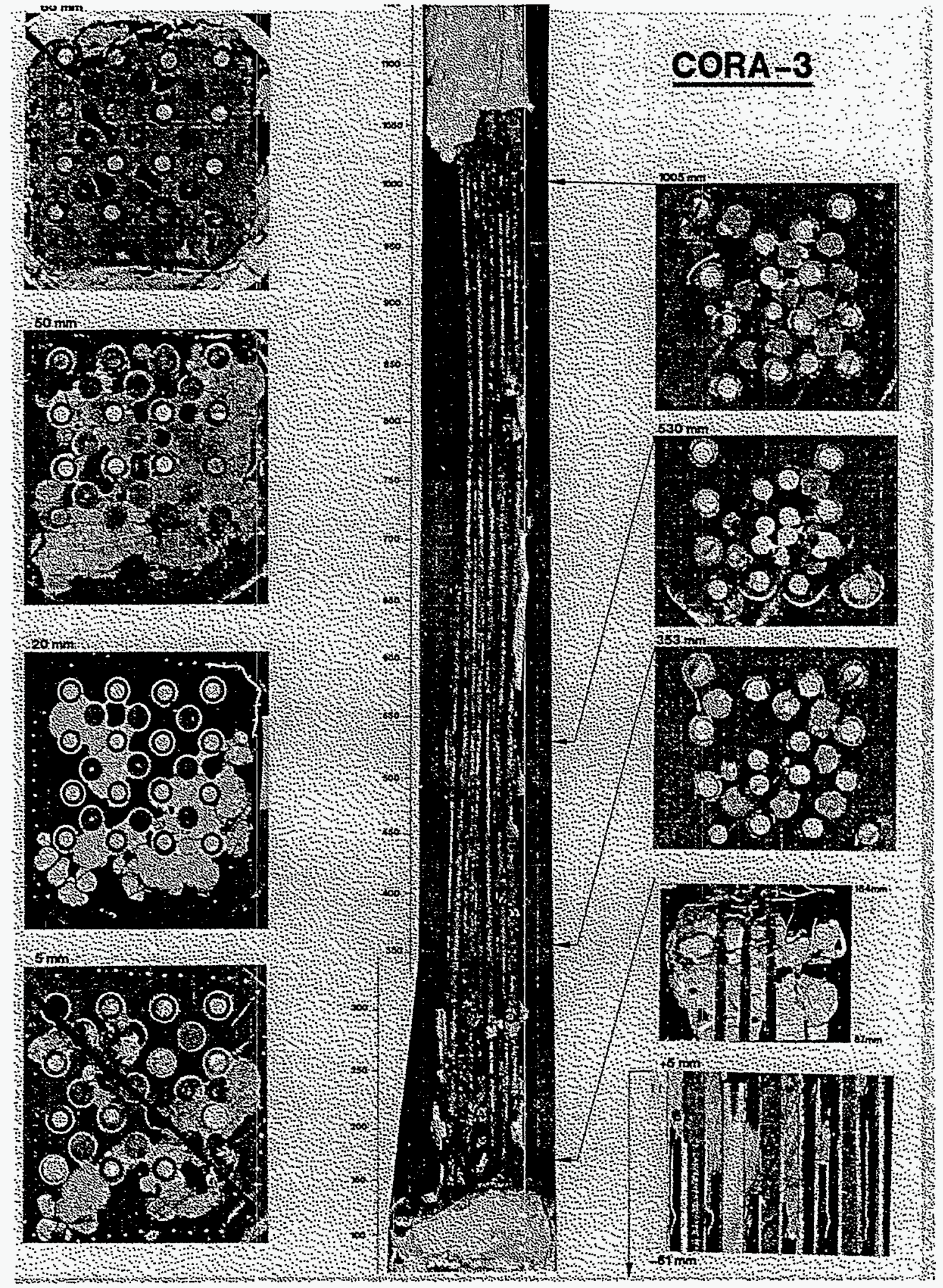

Figure 2-9. Metallographic cross-sections and external view for the CORA-3 experiment. 


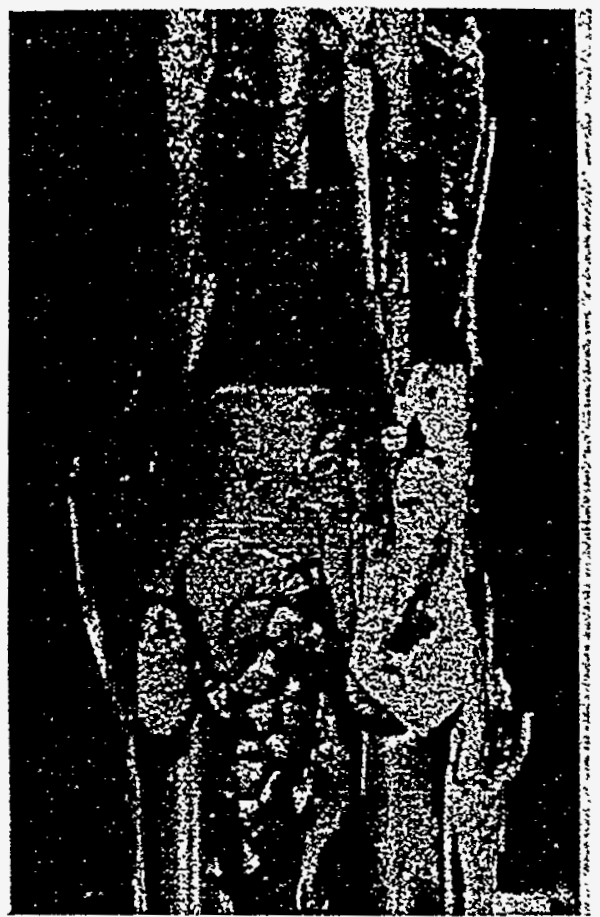

Figure 2-10. Enlargement of the refrozen melt and powdery rubble from ESBU-2A.

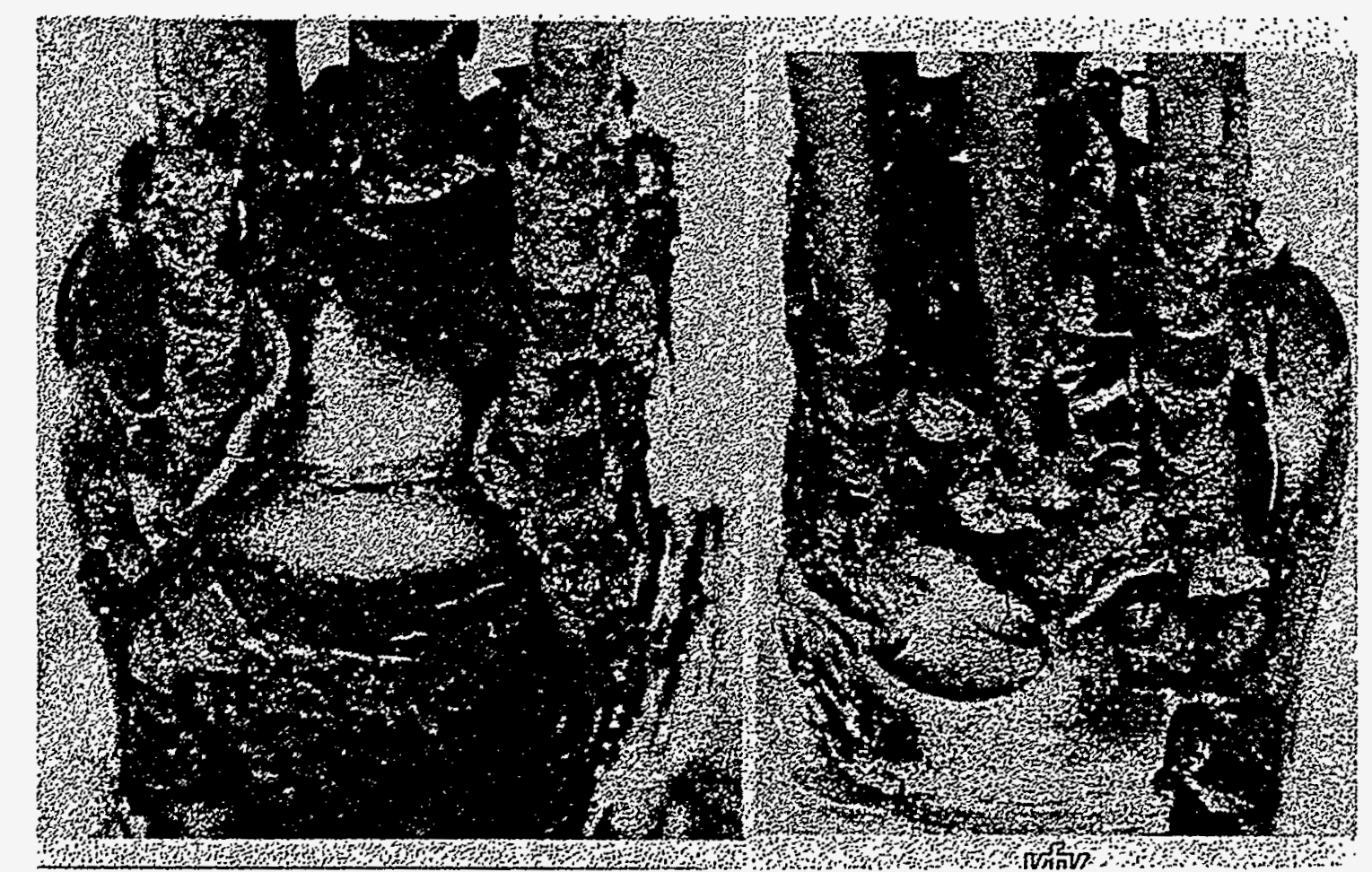

Figure 2-11. Enlarged view of the ESBU-2A blocked region from above: two orientations. 


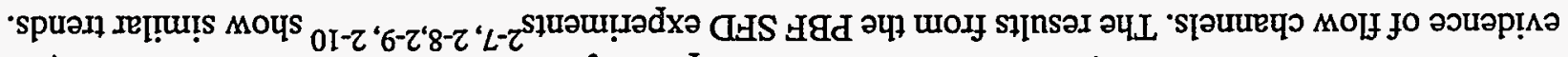

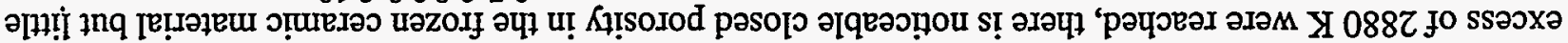

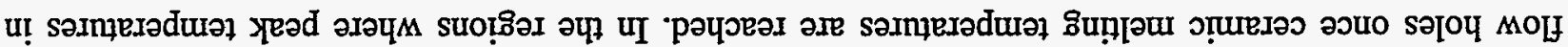

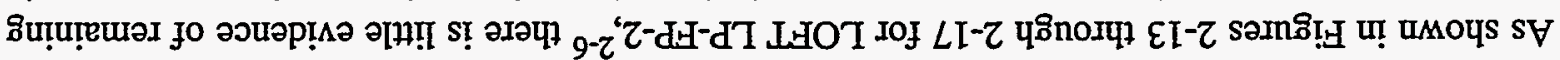

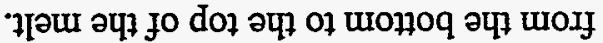

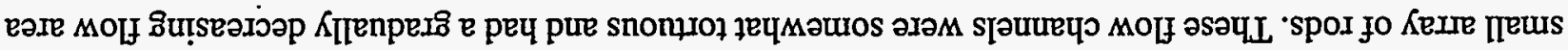

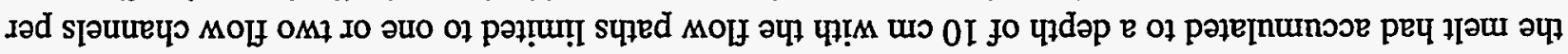

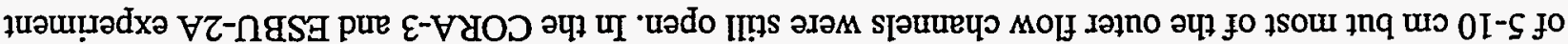

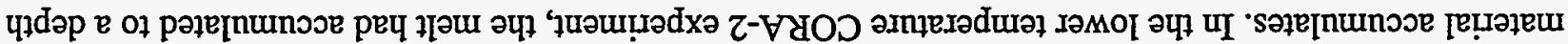

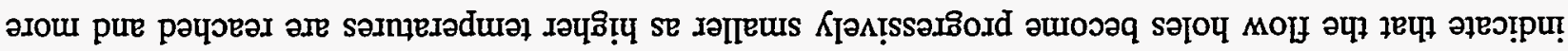

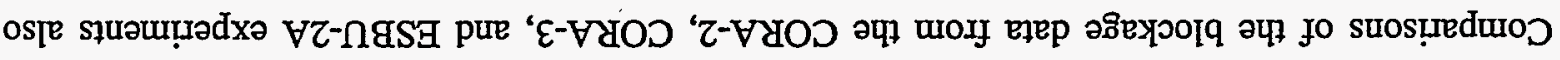

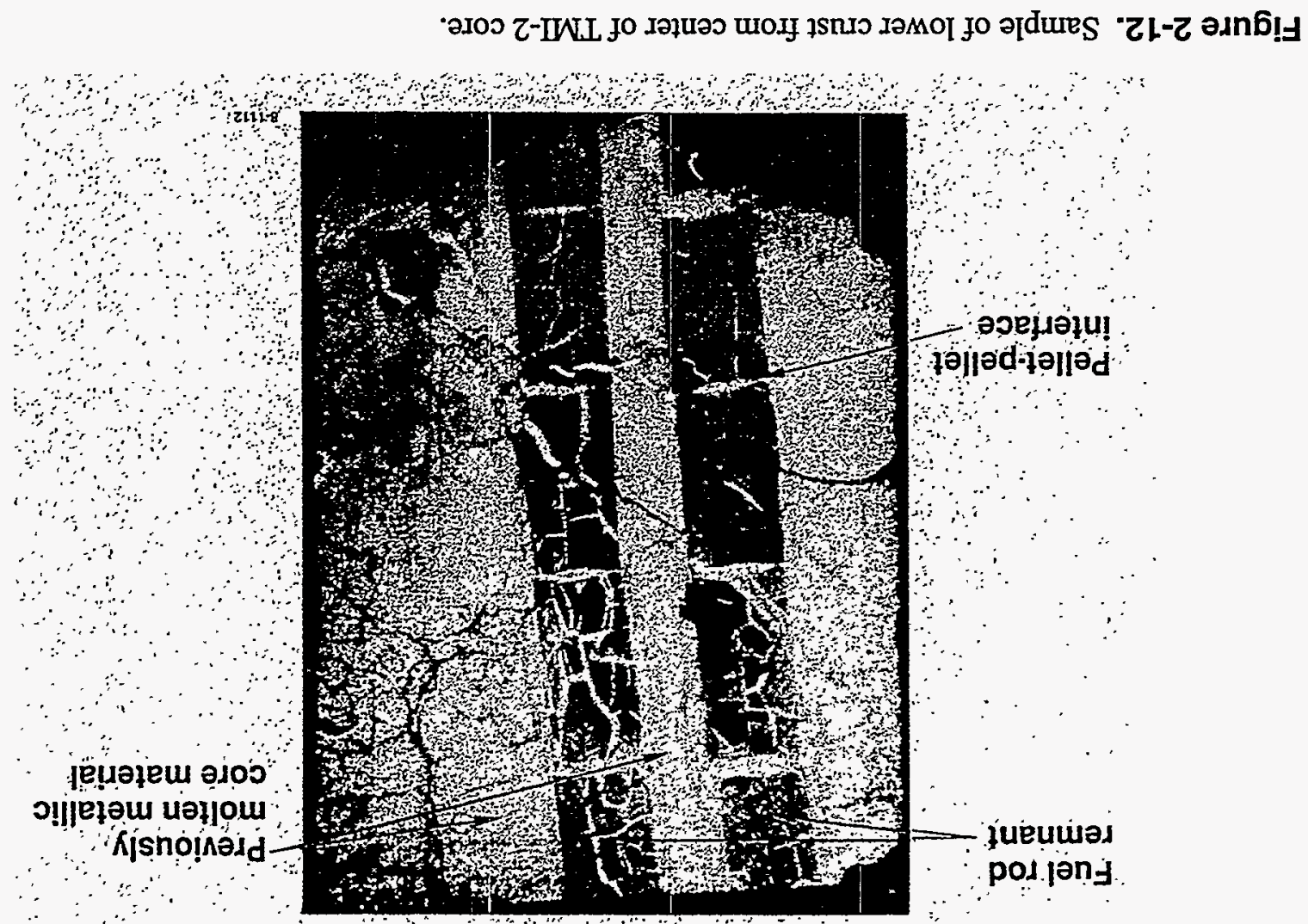




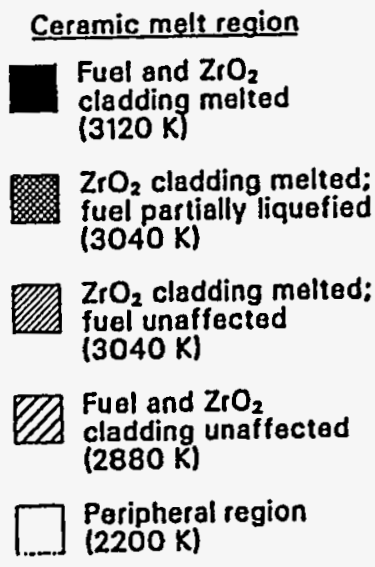

\$220.WHT-480-15

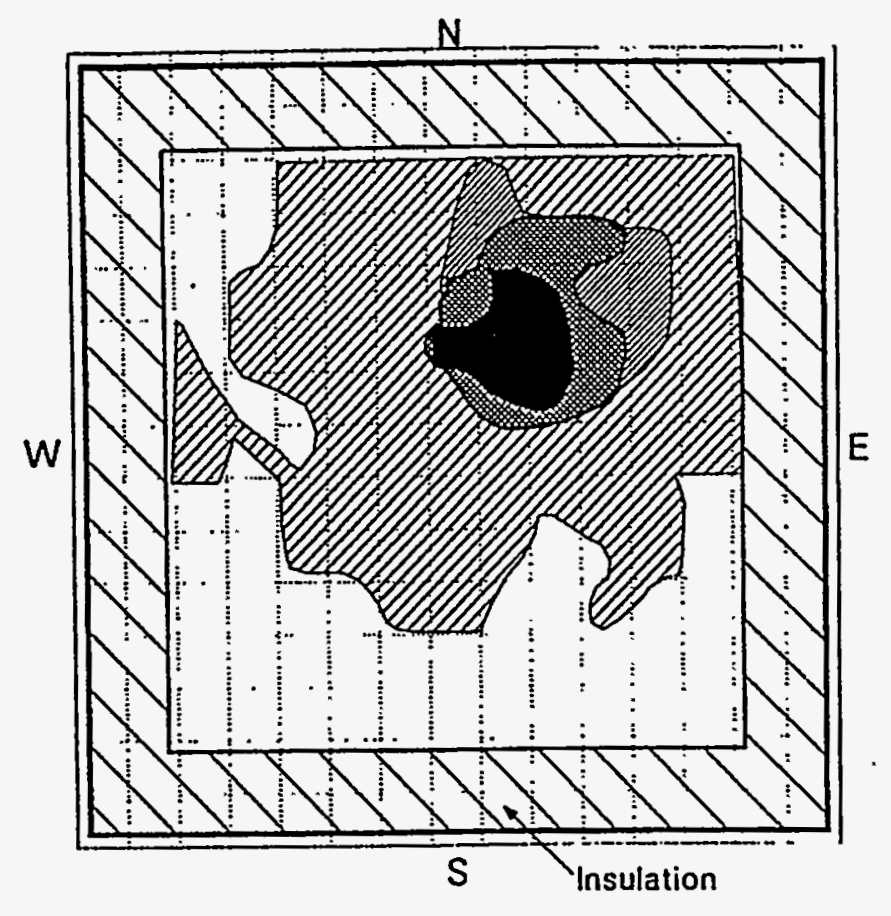

Figure 2-13. Ceramic melt temperatures were based on weighted values.

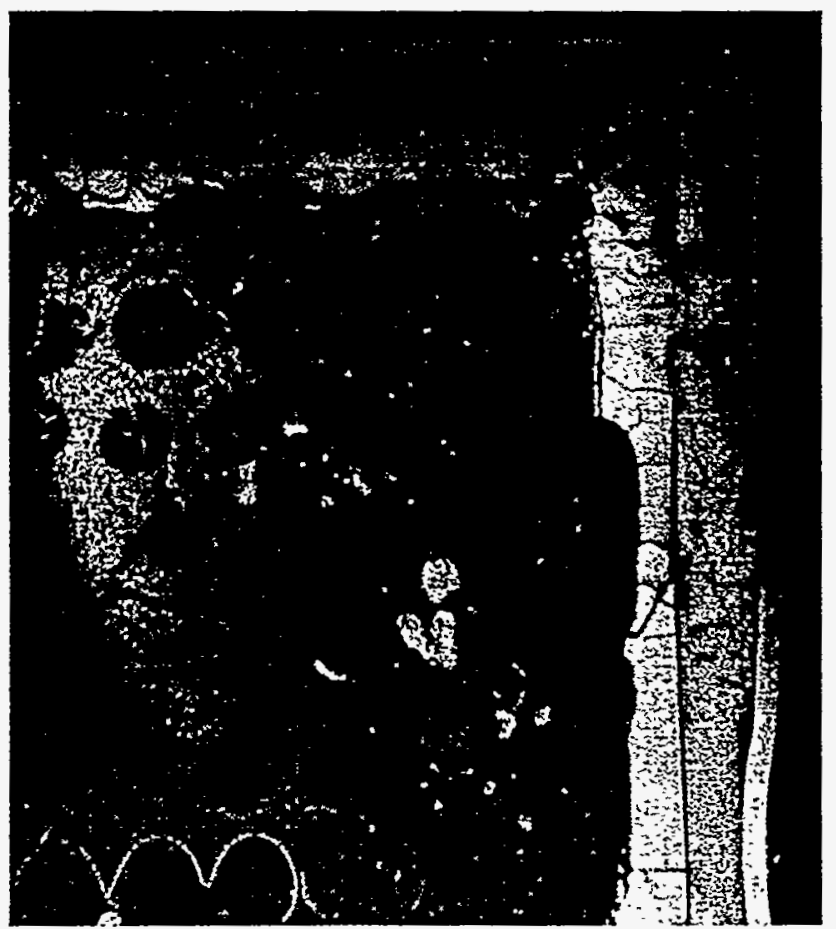

Figure 2-14. LOFT LP-FP-2 metallographic cross-section from the region where a ceramic blockage occurred (NE corner). 


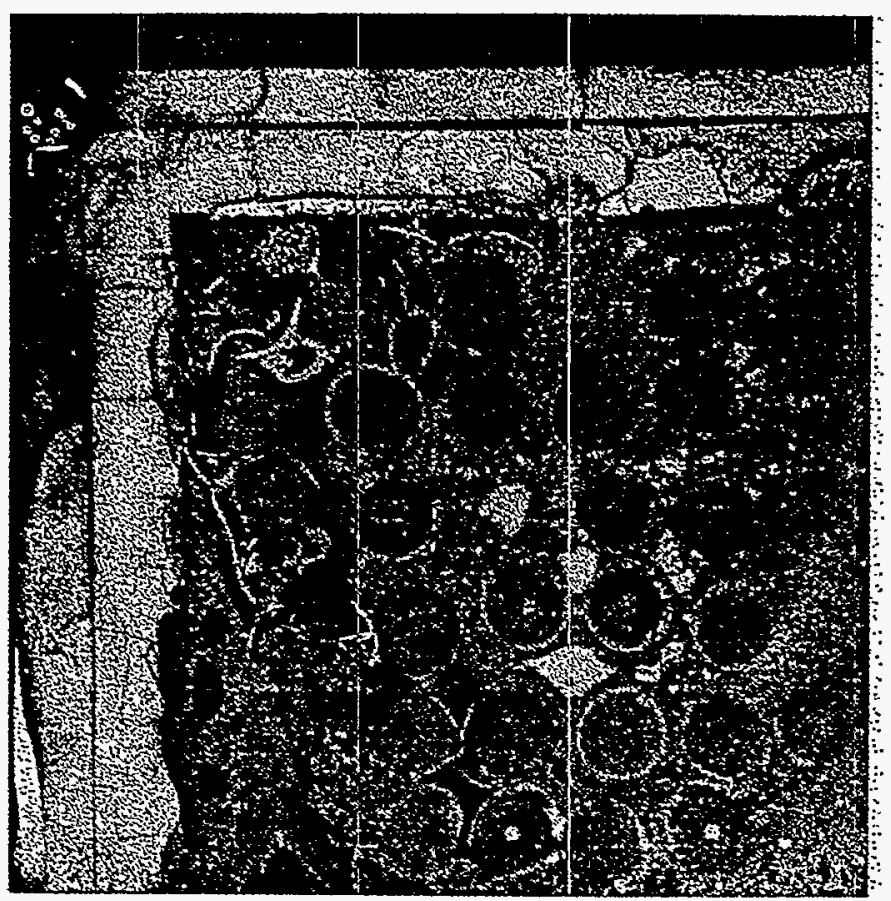

Figure 2-15. LOFT LP-FP-2 metallographic cross-section from the region where a ceramic blockage occurred (NW corner).

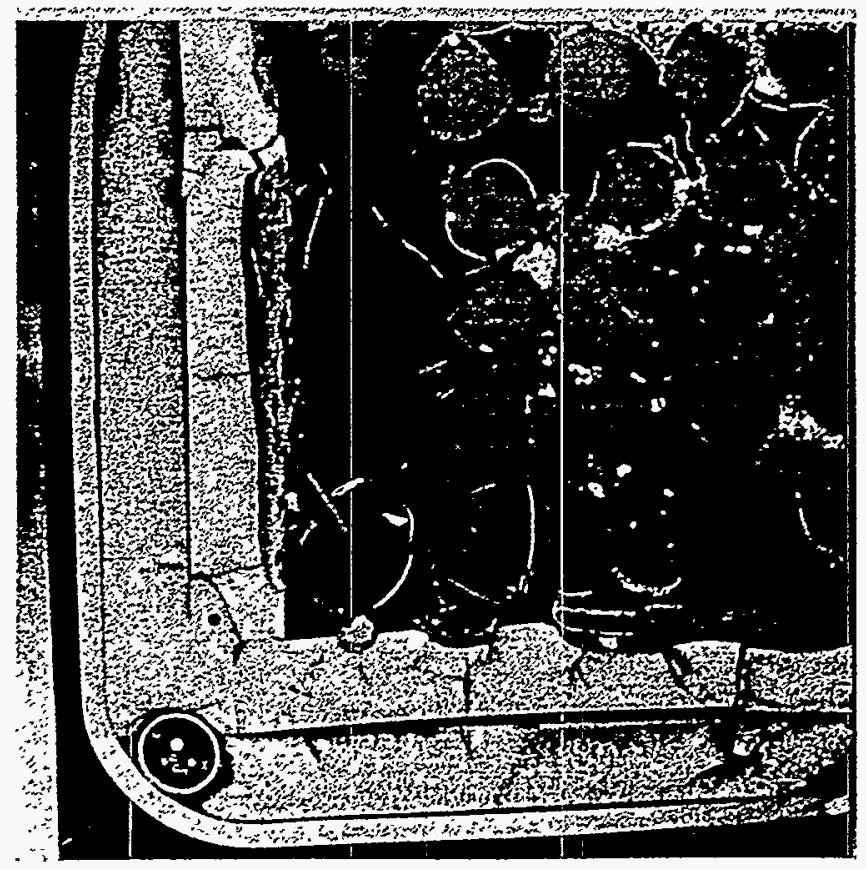

Figure 2-16. LOFT LP-FP-2 metallographic cross-section from the region where ceramic blockage occurred (SW corner). 


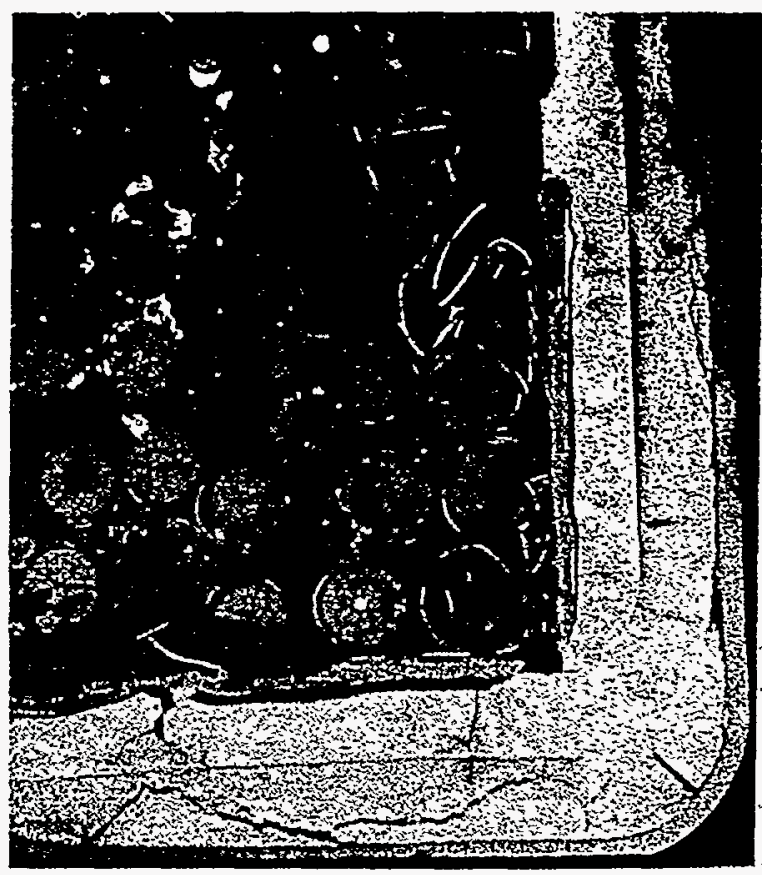

Figure 2-17. LOFT LP-FP-2 metallographic cross-section from the region where a ceramic blockage occurred (SE corner).

The isolated flow holes are also less likely in regions where significant mixing of fuel and control rod materials occur due to the formation of highly porous, foamy melts at temperatures of $2200-2300 \mathrm{~K}$ or below. As shown in the horizontal cross-sections for CORA-5 ${ }^{2-3}$ (Figure 2-18), an experiment like CORA2 but with stainless steel clad, Ag-In-Cd control rods included in the fuel bundle, the frozen material over much of the bundle cross-section is highly porous. As shown in the enlarged vertical cross-section in Figure 2-19, much of the porosity is closed in the vicinity of the original control rod position. The porosity also appears to be insensitive to the type of absorber material present (stainless steel cladding is present in all cases). As shown in Figure 2-20 and Figure 2-21, the porosity in the frozen material for DF-4, $2-11,2-12$ an experiment with stainless steel and $\mathrm{B}_{4} \mathrm{C}$ control blades, appears very much like the melt from CORA-5 with stainless steel and Ag-In-Cd. However, it can not be always assumed that frozen crusts with stainless steel and control material mixed with Zircaloy and fuel are always highly porous. In cross-sections from the TMI-2 lower crust (Figure 2-12), very little porosity is visible. Very little porosity also is visible in the cross-sections from the metallic melt regions from the LOFT LP-FP-2 and SFD 1-4 experiments, although the density of the melt is less than would be expected for Zircaloy, silver, or stainless steel. 


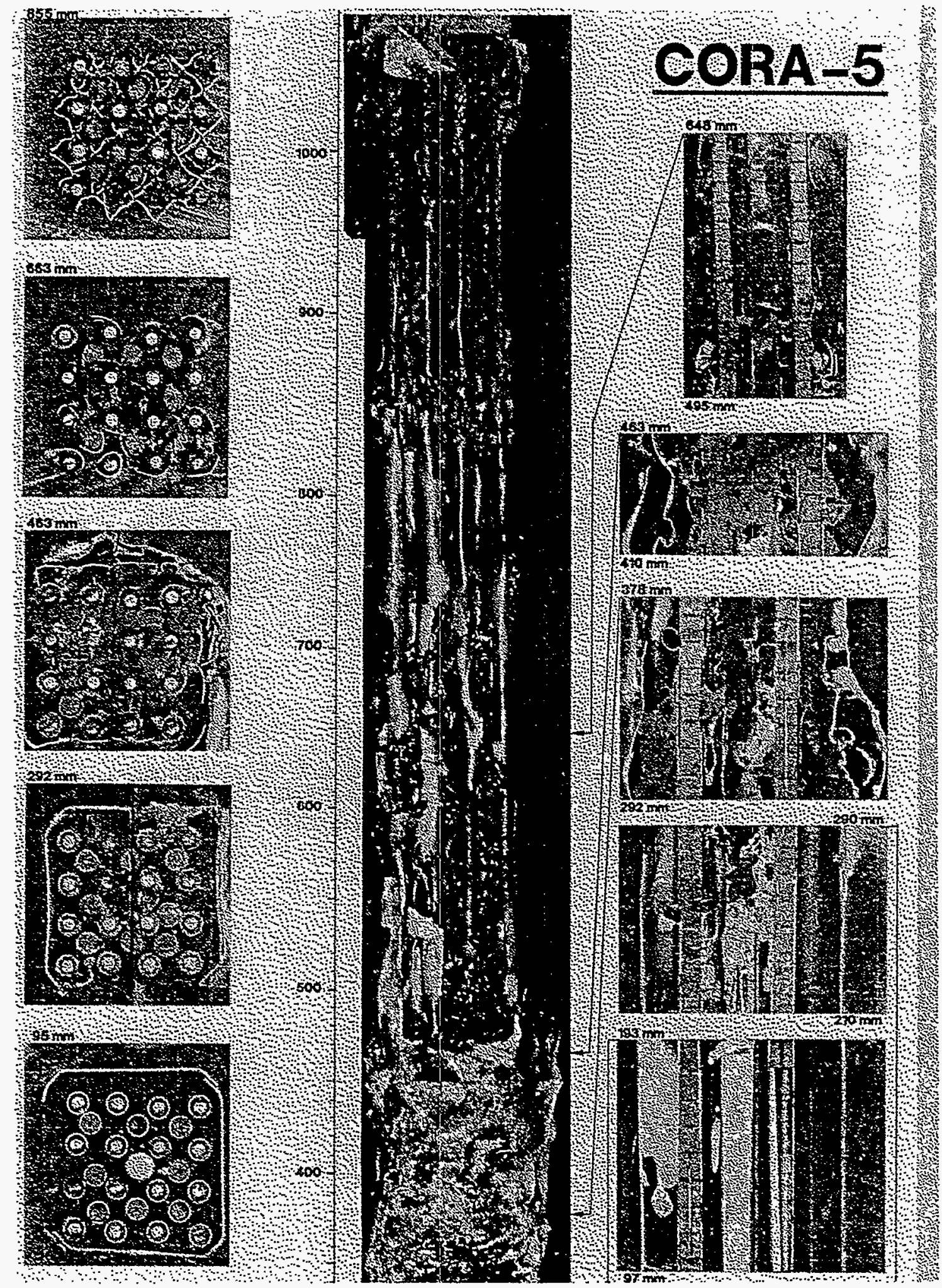

Figure 2-18. Metallographic cross-sections and external view for the CORA-5 experiment. 


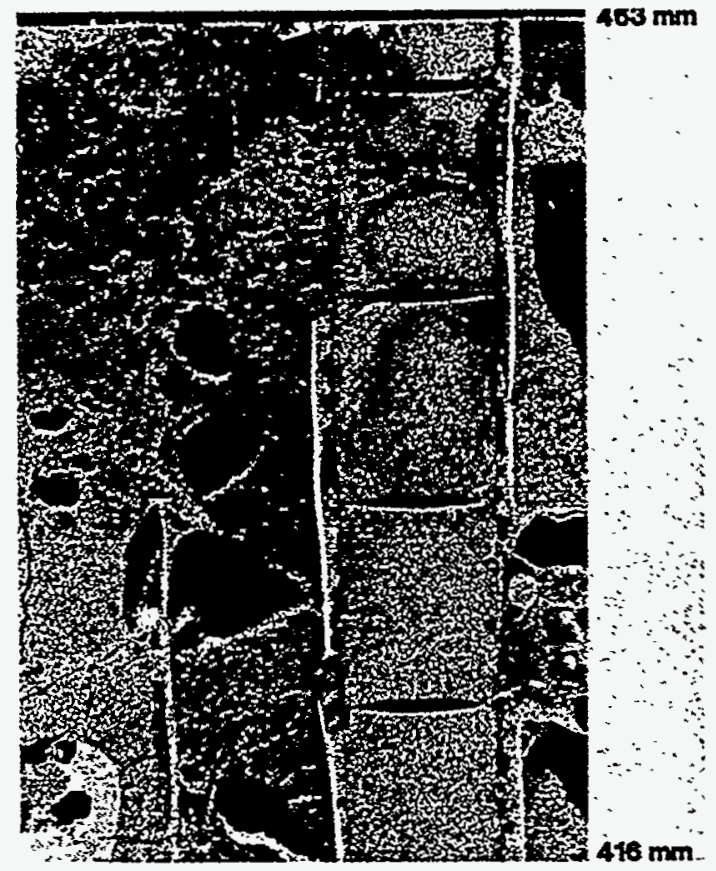

Figure 2-19. Close-up metallographic cross-sectional view of the porosity in the $\mathrm{Zr}$-In-ss blockage in the CORA-5 experiment.

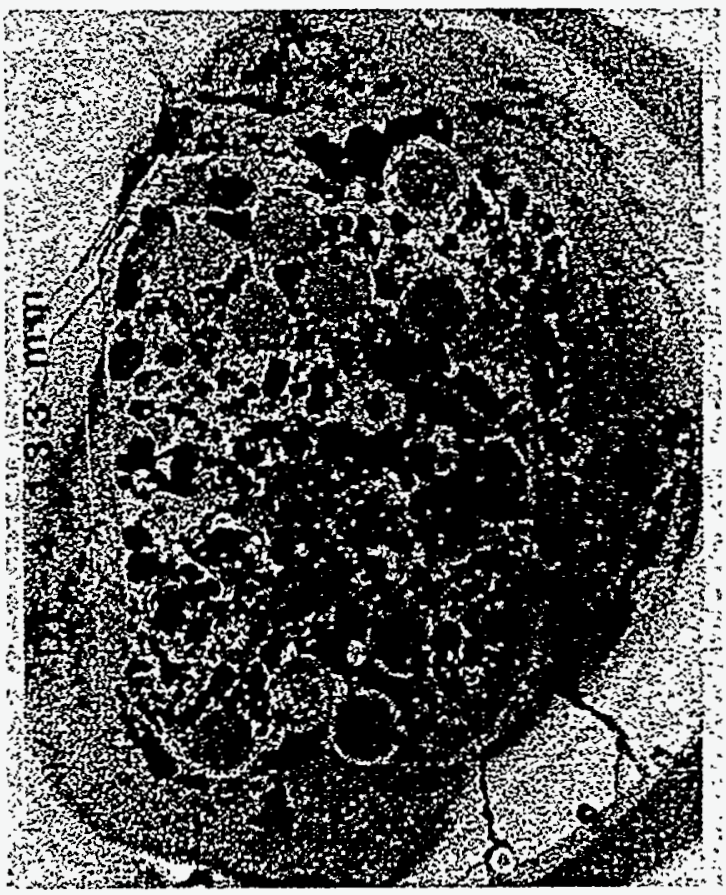

Figure 2-20. Close-up metallographic cross-sectional view of the porosity in the upper portion of the $\mathrm{Zr}$ $\mathrm{B}_{4} \mathrm{C}$-ss blockage in the DF-4 experiment. 


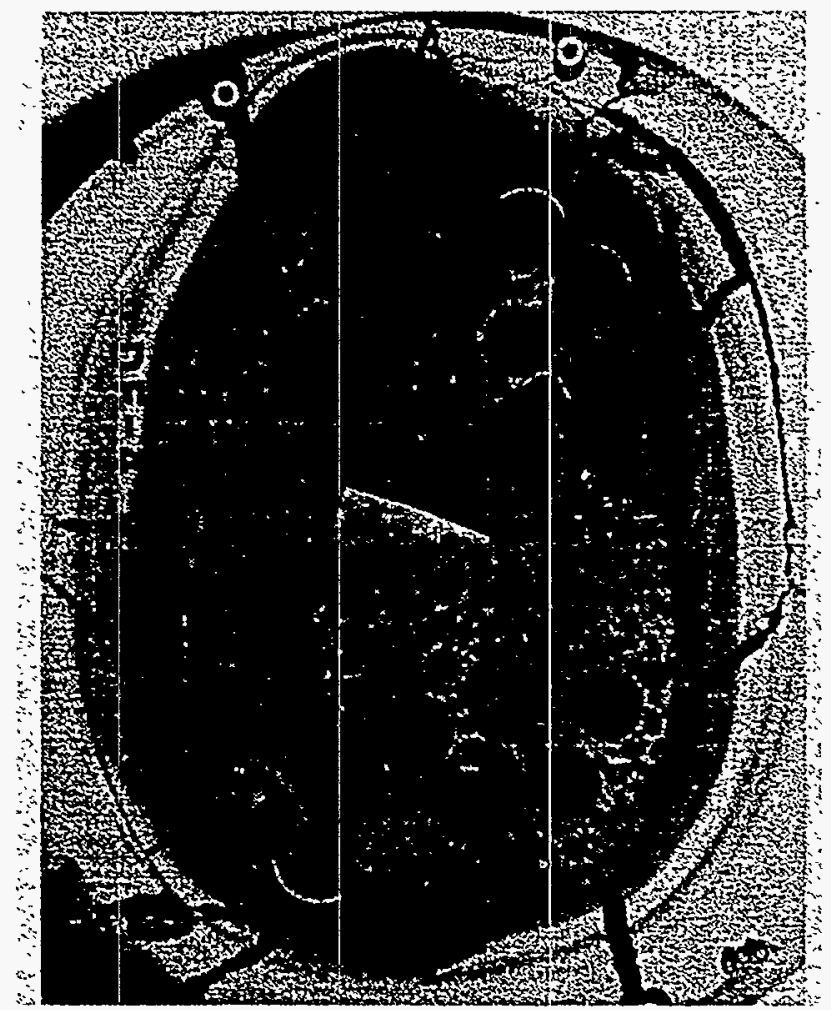

Figure 2-21. Close-up metallographic cross-sectional view of the porosity in a middle portion of the $\mathrm{Zr}$ $\mathrm{B}_{4} \mathrm{C}$-ss blockage in the $\mathrm{DF}-4$ experiment.

The formation of loose debris varied substantially between the different experiments. Loose debris was formed during the slow cooldown phases in the ESBU-2A experiment (Figure 2-22), CORA-2, (Figures 2-6 and 2-7), and PBF SFD 1-4 (Figure 2-23). The loose debris was very fine, desintered fuel grains, in the ESBU-2A, and relatively large in the other two experiments. However, loose debris was not formed in CORA-3, Figures 2-8 and 2-9, and other PBF experiments that were slowly cooled. Loose debris was also formed in the experiments that were rapidly quenched. Representative cross-sections are shown in Figures 2-24, 2-25, and 2-26 for CORA-12, an experiment with stainless steel, Ag-In-Cd control rods, and Figure 2-27 for CORA-17, an experiment with stainless steel, $\mathrm{B}_{4} \mathrm{C}$ control blades. Interesting contrasts can be seen in comparing the cross-sections from CORA-5 and CORA-12 where the only significant difference is the cooling rate. In the bundle views in Figures $2-18$ and 2-24, there is not a dramatic difference in the overall appearance of the bundles although there is a small amount of fragmented material in CORA-12, but not in CORA-5. If the closeup view is taken, there is more of a contrast. The blockage in CORA-5 (Figure 2-19) retains a porous appearance with a few fine cracks, while similar views for CORA12 (Figures 2-25 and 2-26), show that the blockage is much more extensively cracked although the overall integrity of the blockage is maintained. This heavy cracking is also apparent in the cross-section taken through the fuel rods in CORA-12 (Figures 2-25 and 2-26). A similar contrast can be made for the experiments with stainless steel and $\mathrm{B}_{4} \mathrm{C}$ control blades. The cross-section from CORA-17 (Figure 2-27) can be contrasted to CORA-16 (Figure 2-28), an experiment identical to CORA-17 except it was slowly cooled. Like the experiment with $\mathrm{Ag}$-In-Cd, the quenched bundle shows some fragmentation relative to the entrenched bundle but there is still significant amounts of fuel, cladding, and control blade material that remain intact. 


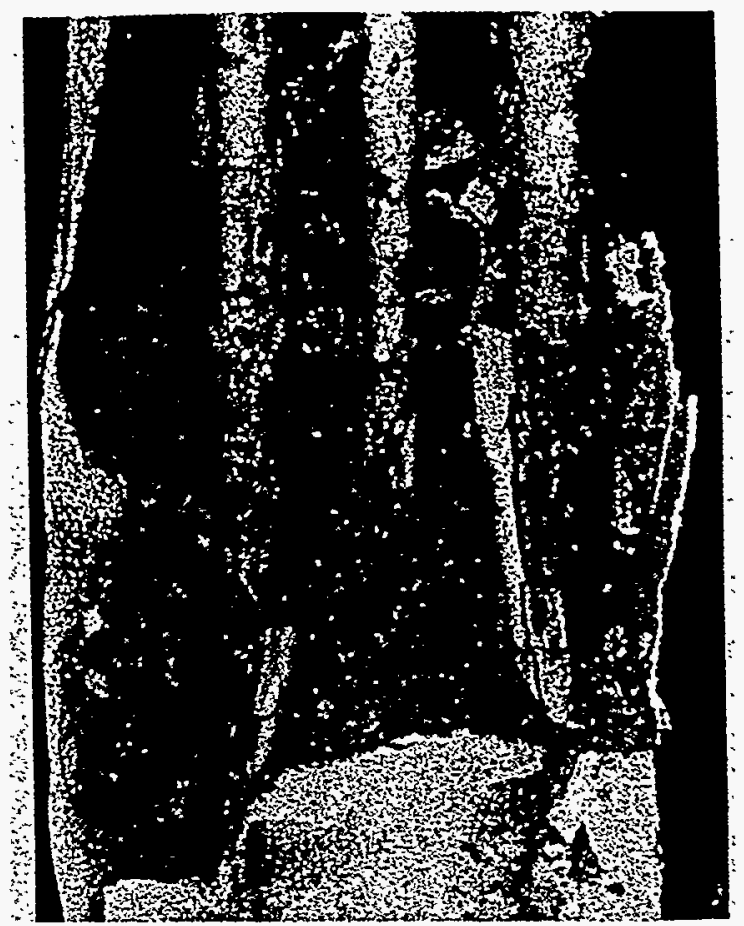

Figure 2-22. Enlargement of the rubble found above the refrozen melt in the ESBU-2A experiment.

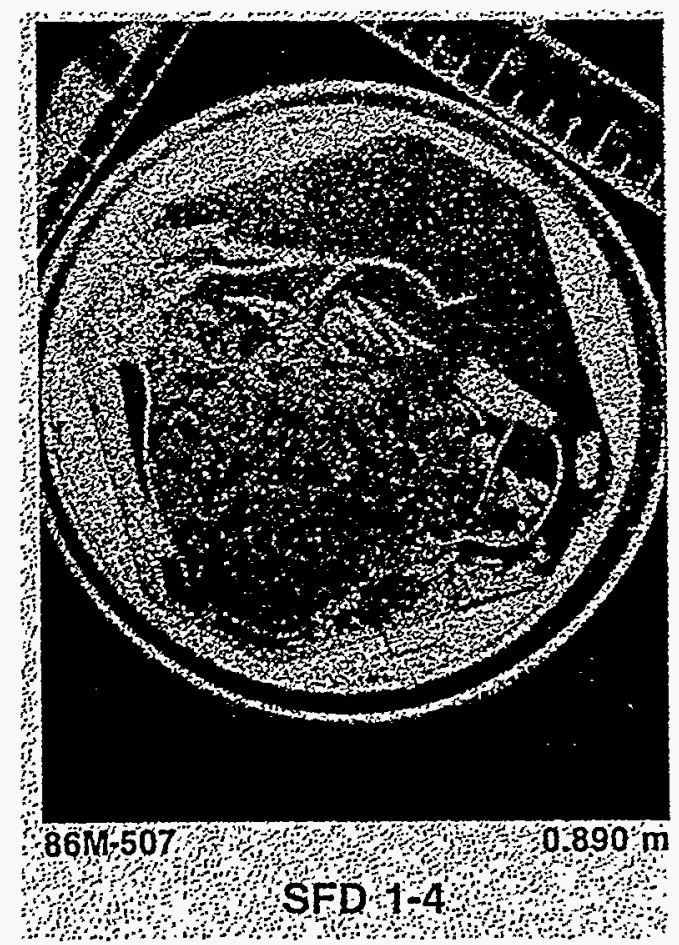

Figure 2-23. Metallographic cross-sections from the PBF SFD 1-4 experiment. 


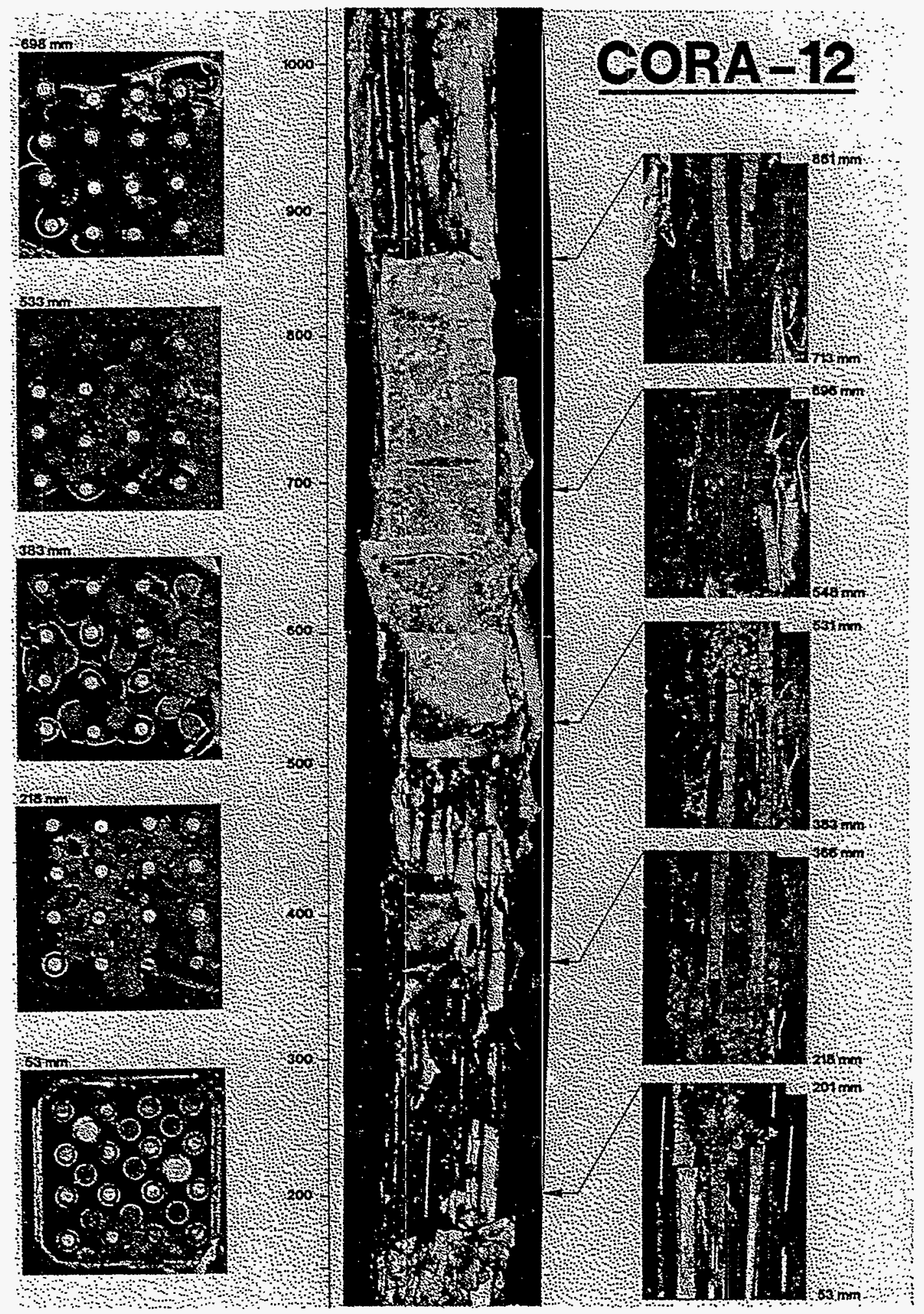

Figure 2-24. Metallographic cross-sections and external view from the CORA-12 experiment. 


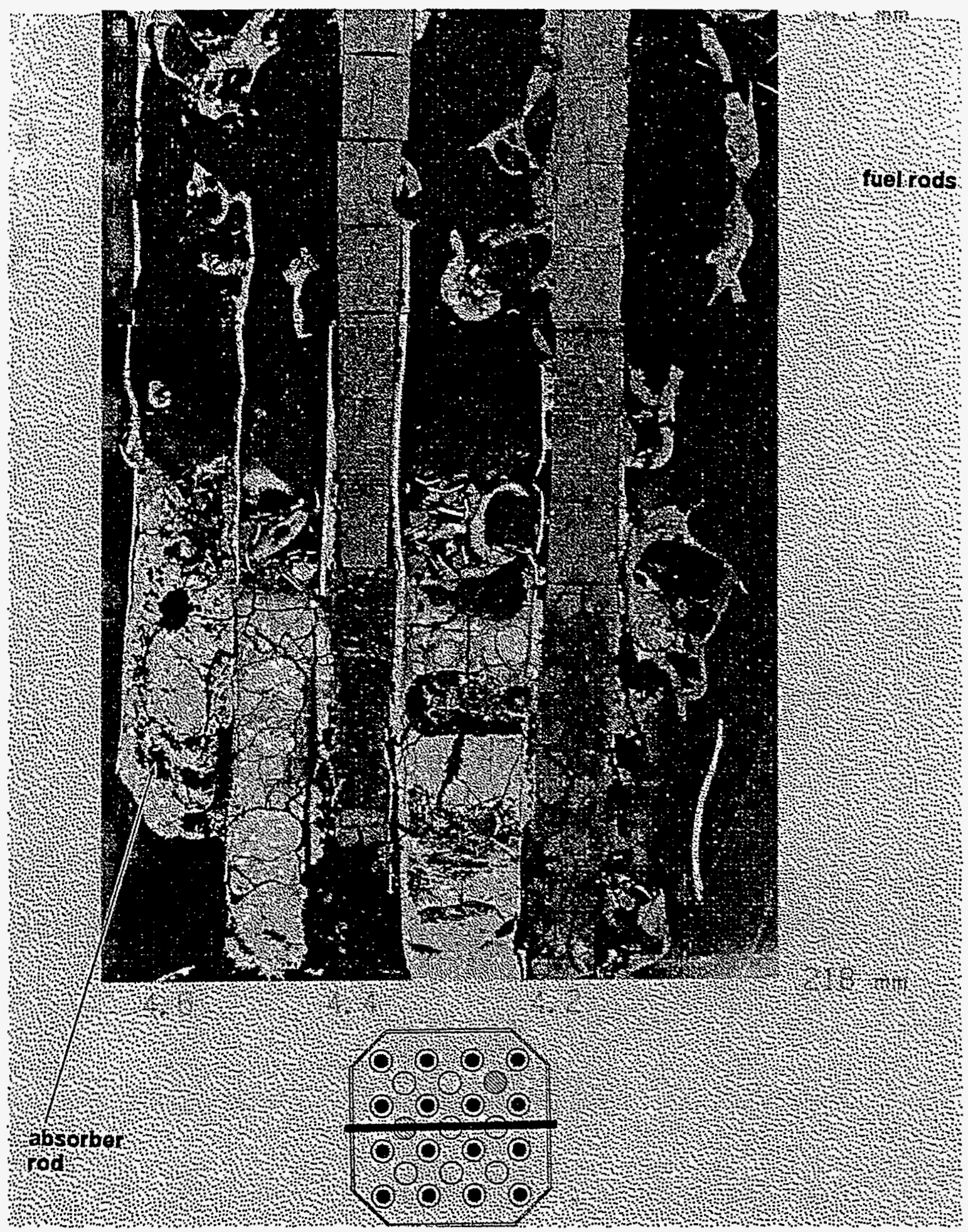

Figure 2-25. Closeup metallographic cross-sectional view from the $\mathrm{Zr}$-Ag-ss blockage in the CORA-12 experiment. 


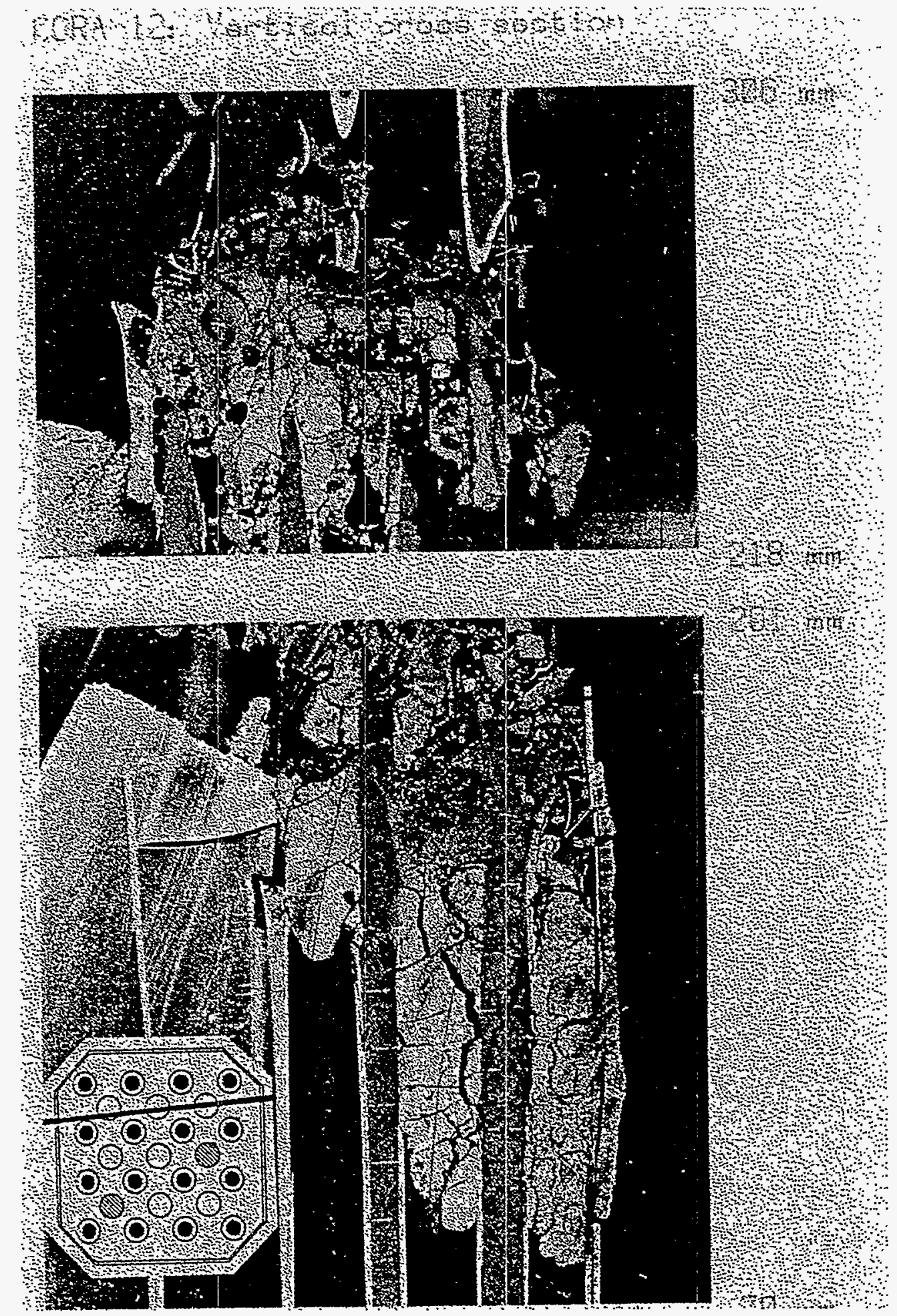

Figure 2-26. Closeup metallographic cross-sectional view of the blockage region in the CORA-12 experiment. 


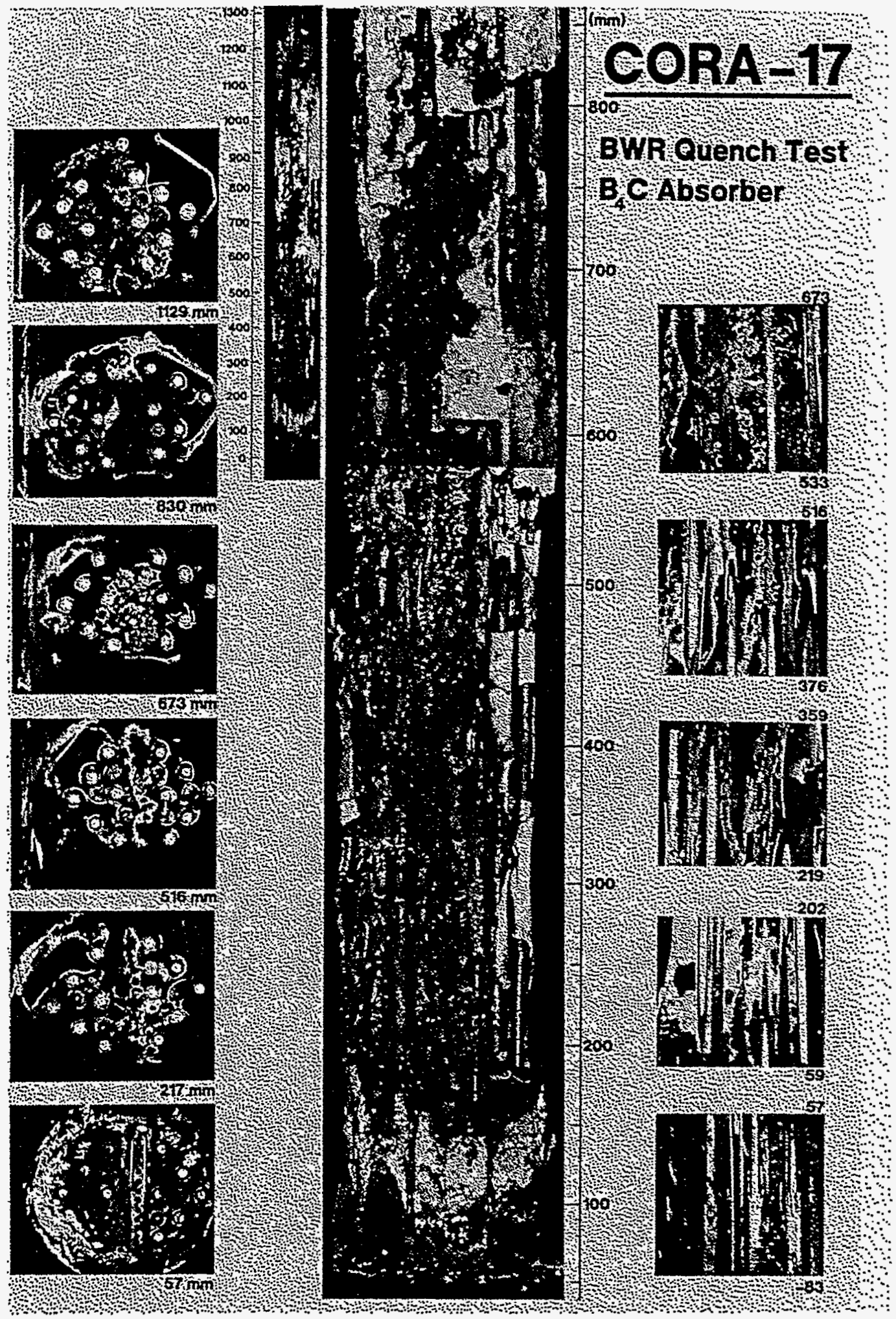

Figure 2-27. Metallographic cross-sections and external view for the CORA-17 experiment. 


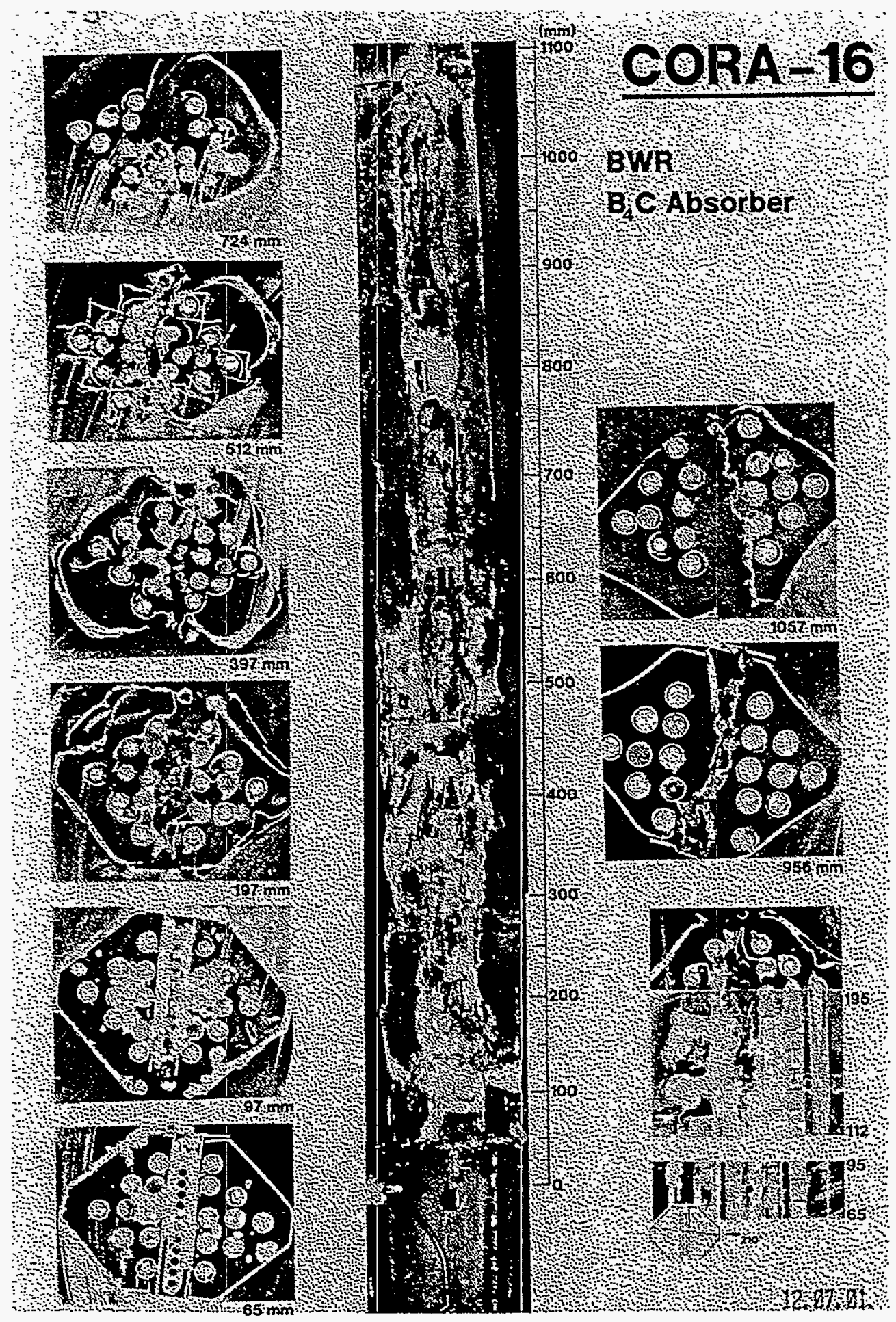

Figure 2-28. Metallographic cross-sections and external view from the CORA-16 experiment. 


\subsection{Review of Existing In-Core and Lower Plenum Debris Models}

The in-core and lower plenum debris models are described in detail in Volume II of the SCDAP/ RELAP5 code manuals. ${ }^{2-13}$ However, the highlights of the models are summarized in this section. Lumped parameter models are used in the core region so that each thermal-hydraulic volume in the core has a corresponding state - either intact components, intact components with a metallic blockage, rubble debris, or a metallic ceramic cohesive debris. In the lower plenum, a detailed 2D model is used. Each element in the mesh can have a different state and composition. The mesh is typically much finer than the thermal-hydraulic mesh.

In the core region, the temperature in the two intact configurations with and without a metallic blockage are computed using individual component $1 \mathrm{D}$ or $2 \mathrm{D}$ heat conduction models. These models will not be discussed in this report. The temperatures in the rubble debris beds are computed using a lumped parameter model so the rubble in each thermal hydraulic volume has a single temperature using a mass average specific heat and heat generation rate. Convective heat transfer from the debris to the coolant is the only heat transfer mechanism considered. An integral method is used to compute the temperature profile in a metallic or cohesive debris region. The temperature profile is assumed to be parabolic in the axial elevation. Convective and radiative heat transfer boundary conditions at the top and the bottom of the cohesive debris, or convective heat fluxes from an internal molten pool are used to compute the melt front position and axial temperature distribution within the blockage. Radial heat transfer is neglected. Oxidation and decay heat generation are considered for all of the configurations.

In the lower plenum, a 2D finite element model is used with an arbitrary, user defined mesh. Convective and radiative boundary conditions can be applied to all exposed surfaces. Heat removed by coolant within the porous debris bed is also considered. Decay and oxidation heat generation are considered.

There are three fundamental aspects to these models which are described in more detail in the next three subsections. First the transition criteria used to form debris regions within the core and lower plenum are discussed. Second, the characteristics of the debris beds are discussed. Third the effective thermal conductivity models used to represent heat transfer within porous debris in the lower plenum are discussed. Effective thermal conductivity models are not used in the core region since debris bed temperatures are computed using lumped parameter models.

\subsubsection{Transition Criteria for Debris Bed Formation}

The transition criteria for debris bed formation are shown schematically in Figures 2-29, 2-30, and 231. The criteria for the transition between an intact configuration to a rubble bed are shown in Figure 2-29. Rubble debris is formed if either (a) embrittled fuel rods (thickness of the cladding $\beta$-layer, $\Delta \mathrm{r}_{\beta}<0.1 \mathrm{~mm}$ ) cools below a fragmentation temperature (default is $\mathrm{T}_{\text {sat }}+100 \mathrm{~K}$ ) or (b) the cladding temperature exceeds the melting point of Zircaloy $\left(\mathrm{T}_{\mathrm{zr}_{\text {mell }}}\right)$ while the cladding oxide thickness is less than $1 \times 10^{-5} \mathrm{~m}$. A cohesive debris region is formed if either the thickness of the frozen crust as predicted by the fuel rod melt relocation models exceed $5 \mathrm{~cm}$ (Figure 2-30), or the $(\mathrm{U}, \mathrm{Zr}) \mathrm{O}_{2}$ melting temperature $(2870 \mathrm{~K})$ is reached (Figure 2-31). In the latter case, molten pool models are also invoked to determine the natural circulation in the molten pool. Adjacent regions of debris are also combined on a thermal-hydraulic volume by volume basis. 

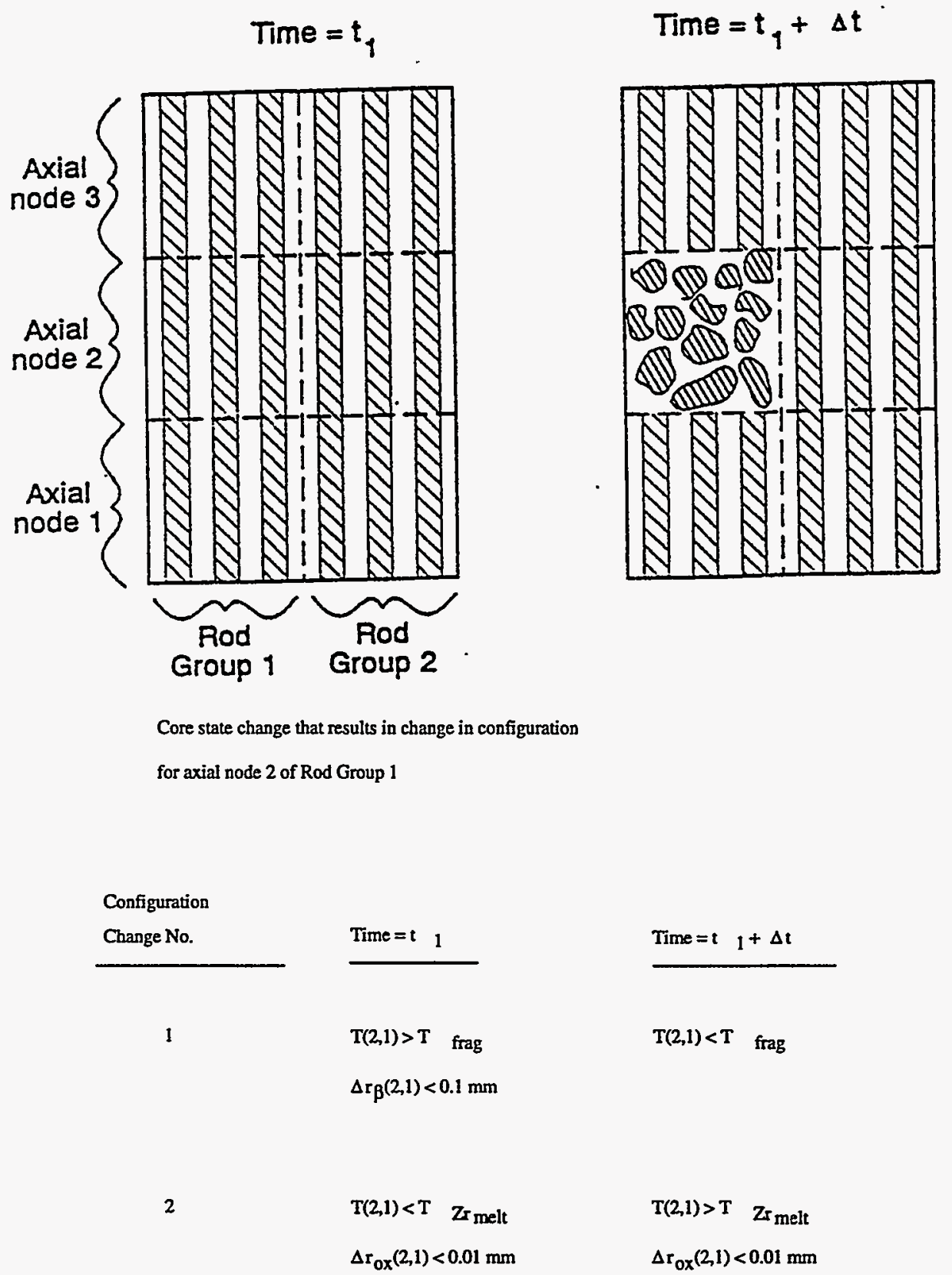

Figure 2-29. Transition criteria for intact to rubble debris geometry. 

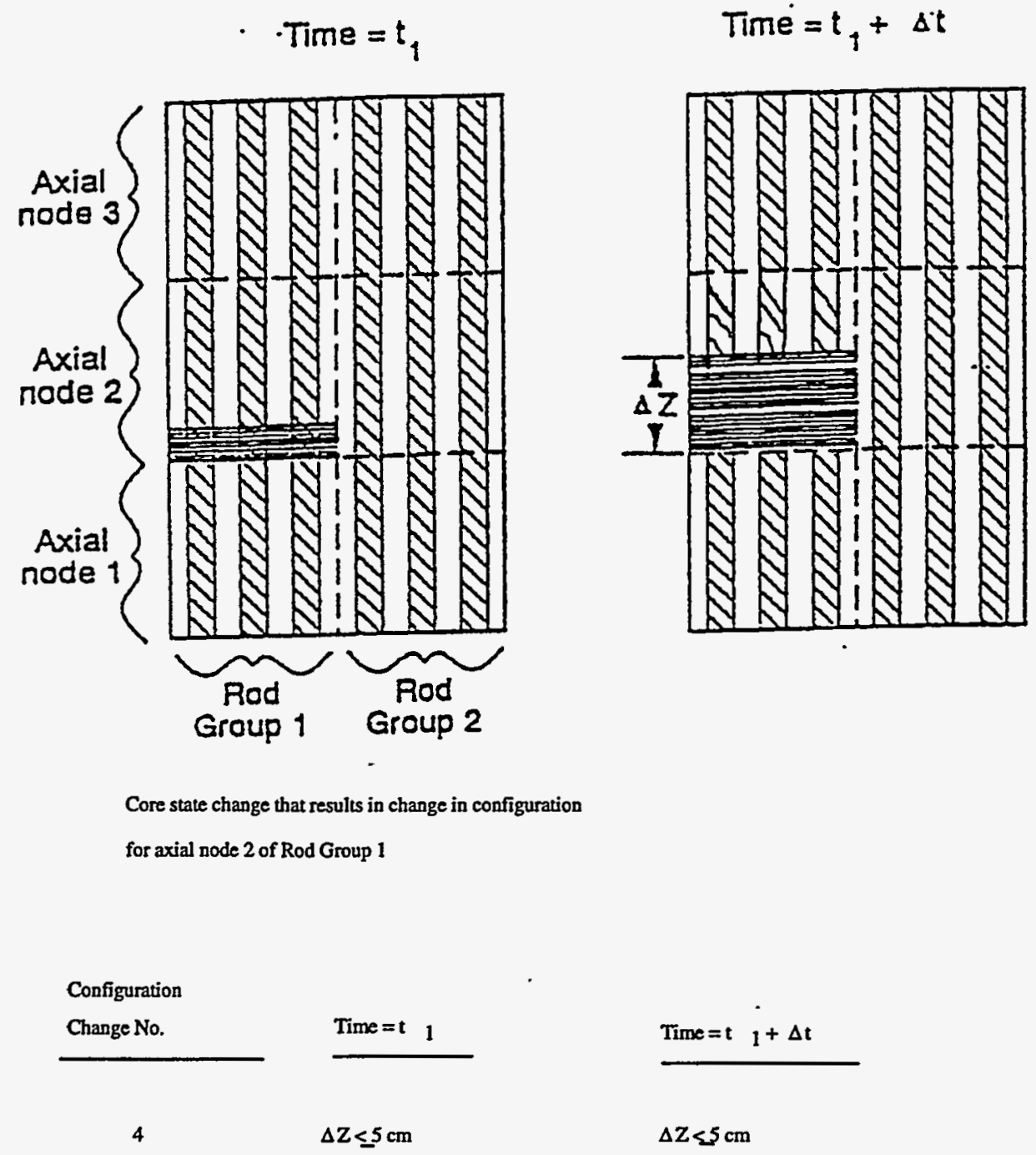

Figure 2-30. Transition criteria for intact to metallic blockage geometry. 
Time $=\mathrm{t}_{1}$

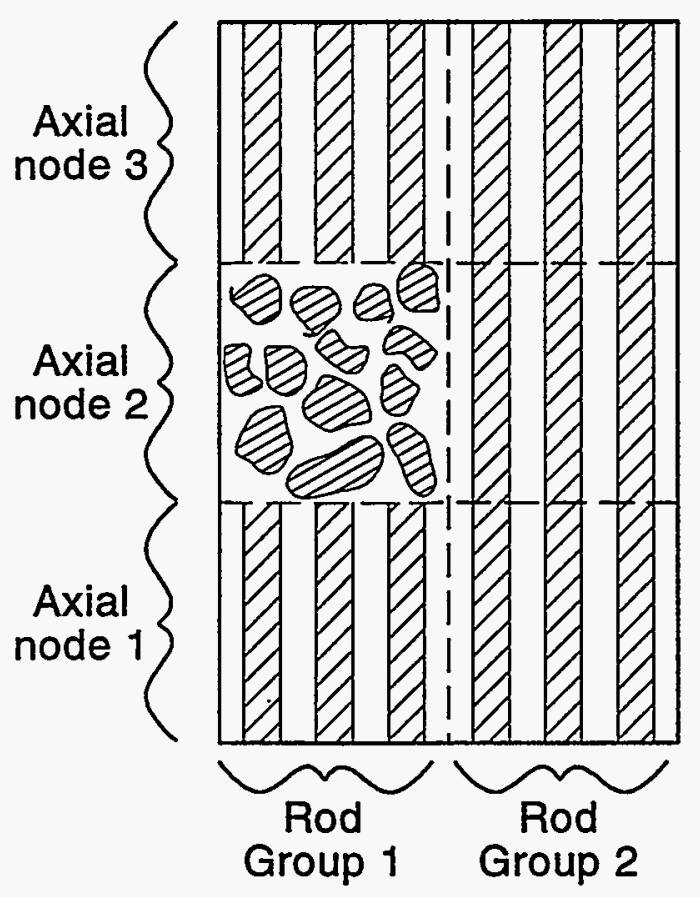

Core state change that results in change in configuration for axial node 2 of Rod Group 1

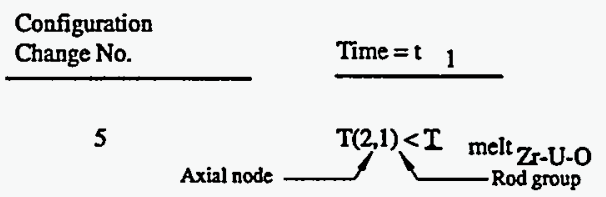

$$
\text { Time }=\mathrm{t}_{1}+\Delta \mathrm{t}
$$

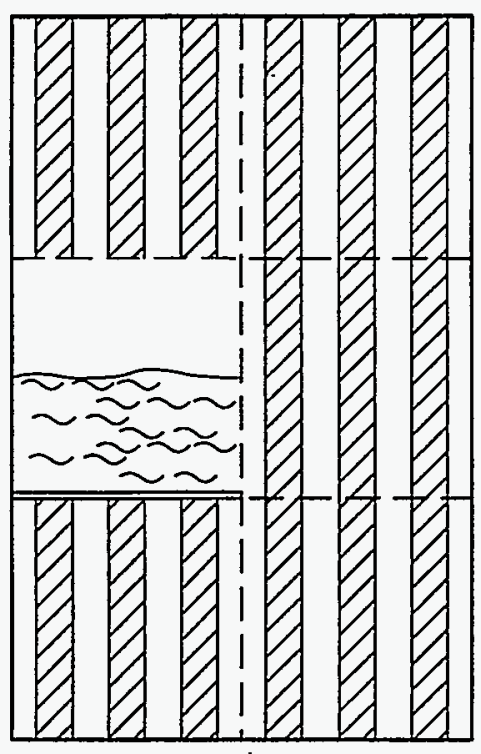

M369-0294-007R
Time $=t \quad 1+\Delta t$

$\mathrm{T}(2,1)>\mathrm{T}_{\text {melt }} \mathrm{Zr- \textrm {U } - \mathrm { O }}$

Figure 2-31. Transition criteria for rubble debris or intact geometries to ceramic melt geometry.

In the lower plenum, debris beds are formed as material relocates into the lower plenum from either the relocation of material from intact components or the slumping of a molten pool. A rubble debris bed is formed if the slumping material is assumed to quench. A cohesive molten pool is formed if the slumped material is assumed to relocate without quenching. 


\subsubsection{Debris Bed Characteristics}

The characteristics of the debris beds in the core and lower plenum are based strictly upon the results from the TMI-2 accident. In the core, rubble debris is assumed to form with an average porosity of 0.54 and an average particle diameter of $0.87 \mathrm{~mm}$. In the lower plenum, an average porosity of $50 \%$ and particle size of $10 \mathrm{~mm}$ is used for rubble debris. The porosities and particle sizes are not changed during the heatup and melting of the debris beds. Cohesive debris beds have zero porosity. Melt relocation within the rubble debris regions is not considered although the rubble debris bed will be mixed with underlying molten pools if either (a) the rubble reaches the melting point of $(\mathrm{U}, \mathrm{Zr}) \mathrm{O}_{2}$ or (b) the upper crust on the molten pool thins below $5 \mathrm{~mm}$. A molten pool can also spread radially or axially into a rubble debris.

\subsubsection{Effective Thermal Conductivity for Lower Plenum Loose Debris}

Heat transfer in a dry, porous debris bed involves both conduction and radiation. The overall effective conductivity, $\mathrm{k}_{\text {eff }}$, of the debris bed is estimated using the following relationship based on ImuraTakegoshi ${ }^{2-14}$ model combined with the Vortmeyer ${ }^{2-15}$ radiation model.

$\mathrm{k}_{\mathrm{eff}}=\mathrm{k}_{\mathrm{cond}}+\mathrm{k}_{\mathrm{rad}}$

where

$$
\begin{array}{lll}
k_{\text {cond }} & = & \text { effective conductivity due to conduction, } \mathrm{W} / \mathrm{mK} \\
\mathrm{k}_{\mathrm{rad}} & = & \text { effective conductivity due to radiation, } \mathrm{W} / \mathrm{mK} .
\end{array}
$$

The effective conductivity due to conduction is estimated by applying the following relationships from Imura-Takegoshi ${ }^{2-14}$

$$
\begin{aligned}
& k_{\text {cond }}=k_{f}\left(\Psi+\frac{1-\Psi}{\left.\Phi+\frac{1-\Phi}{v}\right)}\right. \\
& \Phi=0.3 \phi^{1.6} v^{-0.044} \\
& \Psi=\frac{\phi-\Phi}{1-\Phi} \\
& v=\frac{k_{s}}{k_{f}}
\end{aligned}
$$

where 


$$
\begin{array}{lll}
\mathrm{k}_{\mathrm{f}} & = & \text { gas or vapor thermal conductivity, W/mK } \\
\mathrm{k}_{\mathrm{s}} & = & \text { solid particle thermal conductivity, W/mK } \\
\phi & = & \text { debris bed porosity. }
\end{array}
$$

The effective conductivity due to radiation is estimated by applying the following relationship from Vortmeyer ${ }^{2-15}$

$k_{\mathrm{rad}}=4 \eta \sigma \mathrm{d}_{\mathrm{p}} \mathrm{T}^{3}$

where

$$
\begin{array}{lll}
\eta & = & \text { radiation exchange factor } \\
\sigma & = & \text { Stefan Boltzmann constant }\left(5.672 \times 10^{-8} \mathrm{~W} / \mathrm{m}^{2} \mathrm{~K}^{4}\right) \\
\mathrm{d}_{\mathrm{p}} & = & \text { particle effective diameter, } \mathrm{m} \\
\mathrm{T} & = & \text { particle average temperature, } \mathrm{K} .
\end{array}
$$

In Reference 2-15, Vortmeyer compares various relationships for the radiation exchange factor, $\eta$, from several references for a range of particle emissivities and concludes that all of the relationships yield approximately the same numerical values for the radiation exchange factor. Typical values for the radiation exchange factor for a case with a porosity of 0.4 are shown below in Table 2-1 for various emissivities.

Table 2-1. Comparison of estimates for radiation exchange factor $(\phi=0.4)$.

\begin{tabular}{|c|c|c|c|c|c|c|}
\hline$\varepsilon$ & $\begin{array}{c}\text { Argo } \\
\text { and } \\
\text { Smith }^{2-16}\end{array}$ & Schotte $^{2-17}$ & Laubitz $^{2-18}$ & Vortmeyer $^{2-15}$ & $\begin{array}{c}\text { Wakeo } \\
\text { and } \\
\text { Kato }^{2-19}\end{array}$ & Kasparek $^{2-20}$ \\
\hline 0.20 & 0.11 & 0.20 & 0.27 & 0.24 & 0.21 & 0.35 \\
\hline 0.35 & 0.21 & 0.35 & 0.46 & 0.35 & 0.37 & 0.48 \\
\hline 0.60 & 0.43 & 0.60 & 0.80 & 0.59 & 0.65 & 0.72 \\
\hline 0.85 & 0.74 & 0.85 & 1.13 & 0.93 & 0.96 & 0.97 \\
\hline 1.00 & 1.00 & 1.00 & 1.32 & 1.22 & 1.15 & 1.12 \\
\hline
\end{tabular}


Experiments considering a wide range of particle materials (glass, bronze, copper, iron, alumina), sizes $(0.04$ to $3.25 \mathrm{~mm}$ ), and particle bed porosities $(0.29$ to 0.39$)$ and gas or vapor types (helium, Freon12 , air) and pressures ( $1 \times 10^{-4}$ to $\left.0.1 \mathrm{MPa}\right)$ were used to develop the Imura-Takegoshi and Vortmeyer model. The Imura-Takegoshi and Vortmeyer references are also cited as the basis for the debris bed effective conductivity model used in the DEBRIS late-phase melt progression model ${ }^{2-21}$ developed by Sandia National Laboratory (SNL). However, SNL has modified the equation for predicting the effective conductivity associated with radiation to the equation shown below

$\mathrm{k}_{\mathrm{rad}}=4 \varepsilon \sigma \pi \mathrm{d}_{\mathrm{p}} \mathrm{T}^{3} \eta_{\mathrm{sNL}}$

where

$$
\begin{array}{lll}
\varepsilon & =\quad \text { debris particle emissivity } \\
\eta_{\mathrm{SNL}}= & \begin{array}{l}
\text { empirical parameter }\left(\eta_{\mathrm{SNL}}=0.25\right. \text { is the value estimated for the MP } \\
\text { experiments). }
\end{array}
\end{array}
$$

A comparison of the equations used in the SNL DEBRIS and INEL COUPLE models indicates that different equations for the radiation exchange factor were selected in these models. Calculations discussed in Section 2.4 illustrate that results from the INEL and SNL interpretations of the Imura-Takegoshi and Vortmeyer model are significantly different.

\subsection{Proposed Model Improvements}

As described in Section 2.1, the debris beds forming in the core region are expected to be very heterogeneous during the transition from an intact geometry. For example in the LOFT LP-FP-2 center fuel assembly, the debris regions consisted of small masses of both metallic and ceramic material, relatively intact rods with intact or "flowered cladding," grid spacer and other control remnants, and relatively loose debris. The loose debris formed, and ceramic melting occurred during reflood. The crosssectional average open porosity varied from $0 \%$ in the ceramic melting region, to $15 \%$ in the region where the metallic melts had occurred, to nearly $75 \%$ where the loose debris had accumulated. The heterogeneous nature of the debris beds cannot be adequately described by the existing models. Although the existing models describe ceramic melting regions and relatively intact portions of the bundle, the open porosity in regions where the metallic material freezes can not be described well (it is assumed to be completely blocked in the center of the bundle) and porosity and character particle sizes in the loose debris regions cannot be predicted.

The transition between the different states of the core also cannot be described very well by the existing models because a very abrupt transition is assumed. For the LOFT LP-FP-2 experiment, the weakness of the abrupt transition models was the most evident. As described in the SCDAP/RELAP5/ MOD3.1 assessment documents, ${ }^{2-22}$ the abrupt transition from an intact to ballooned geometry, and to metallic blockage formation resulted in an over prediction of the flow diversion from the central fuel assembly to outer peripheral assemblies. This was resolved by subdividing the central fuel assembly into several parallel flow paths to smooth the transition. However, use of such a fine radial nodalization is 
impractical during the analysis of a full plant. As a result, physically unrealistic blockage and flow diversions have been observed in some full plant calculations.

The diversity of debris bed porosities (and particle sizes for loose rubble) observed in the experiments cannot be described by existing models. In the case of metallic and ceramic blockages, the extensive closed porosity observed in the experiments with control and structural materials is not represented. As a result the volume of the blockages can be underpredicted by as much as $50 \%$ in the most extreme cases. In the case of rubble debris, the possible desintering of the fuel, as was most apparent in the ESBU-2A experiment, and the variation in particle size and porosities observed in the other experiments cannot be described using the single porosity and particle size correlations used in the code. As a result, the volume and thermal-hydraulic characteristics of the rubble debris are not accurately described.

The nature of the debris bed formed in the lower plenum of TMI-2 also cannot be described by the simple porosity and particle size model. Even though this model was originally developed as a result of the very early "grab" samples taken from the TMI-2 lower plenum, subsequent examination and analysis of this debris has indicated that a much more diverse model is required.

However, even though the geometries of the debris beds (and the transition from one geometry to another) are not well characterized by existing models, it is clear that the basic mechanisms leading to the formation of debris beds in the core are described quite well by existing models. The temperatures and time for the formation of (a) metallic blockages due to the interaction between grid spacers, control rod spacers, and fuel rod cladding, and (b) ceramic blockages due to $(\mathrm{U}, \mathrm{Zr}) \mathrm{O}_{2}$ melting are very consistent with the experimentally observed behavior. The ability of existing models to predict the conditions leading to the formation of rubble debris is less clear due to difficulty in interpreting existing experiments, but the basic trends predicted by the models are also consistent.

The ability of the existing models to predict the subsequent behavior of the debris bed is less clear because the data base for debris heating, melting, or quenching is much more limited. As a result, the motivation for developing improved models in this area comes primarily from the desire to develop more mechanistic models, as suggested by the peer reviewers, and to eliminate physically unrealistic behavior observed in full plant calculations.

Consequently, it is proposed that the general framework of the existing incore and lower plenum debris models remain the same as before. That is, in the core region prior to the formation of complete ceramic blockage and molten pools, each thermal hydraulic volume within the core has a corresponding single intact or debris state. For rubble debris in the core a lumped parameter thermal model will be used so that the rubble in each thermal-hydraulic volume has a single temperature, since the heat removed by the coolant within the rubble should be the dominant mode of heat transfer. For metallic or ceramic cohesive debris regions, the heat conduction within the debris would be represented using an integral method. In this way, the temperature profiles and melting fronts can be established. This approach would also apply to rubble debris volumes which have melted and formed a cohesive debris bed. This approach would be modified somewhat once molten pools are formed but an integral approach will still be essential to estimate the temperature gradients in the vicinity of the molten pool crust. However, the transition criteria used to form these debris beds and debris bed characteristics would be modified to better reflect the current data base as discussed in Sections 2.3.1 and 2.3.2. 
In the lower plenum, the existing 2D finite element model would be used. However, the relocation of material within the debris bed once melting has occurred will be included as described in Section 2.3.3 to reflect the changes in initial porosity and volume during melting. In addition, the existing effective thermal conductivity model will be modified as described in Section 2.3.3.

\subsubsection{Transition Criteria for Debris Bed Formation}

The transition criteria for rubble formation will be modified to reflect the following general trends noted in the data discussed in Section 2.1.

(1) The quenching of hot bundles with temperatures in excess of Zircaloy melting also resulted in the formation of a rubble debris bed. However, only portions of the experimental bundles were fragmented. In the CORA-12, CORA-13, CORA-17, and LOFT LP-FP-2 experiments, only the upper portions of the bundles were shattered indicating that the peak temperatures and time at peak temperatures are important factors. In comparing the CORA experiments with LOFT LP-FP-2, the shattering was much more extensive in the LOFT experiment than in the CORA experiments. The primary difference between these two sets of experiments is the peak temperatures reached. The temperatures in the LOFT experiment were much higher than in the CORA experiments. In the LOFT experiments the upper portion of the bundle was above $2400 \mathrm{~K}$ for nearly four minutes while the peak temperatures in the CORA experiments reached only $2150-2250 \mathrm{~K}$ for a similar period of time.

In addition, there was no noticeable shattering of the fuel in regions where (a) the average fuel temperature reached or exceeded the melting temperature of $(\mathrm{U}, \mathrm{Zr}) \mathrm{O}_{2}$, (b) "flowered" cladding remained on the fuel, or (c) noticeable quantities of metallic or ceramic frozen materials were observed. It should be noted that the fuel was extensively cracked in these regions where "flowered" cladding or metallic frozen material were present but the fragmented fuel was held in place by the cladding or frozen material. In the case of the flowered cladding, the cladding was intact lower in the bundle although the peak temperatures were the same as the upper portion of the bundle where there were no remnants of cladding. In all cases the remnants of zircaloy examined were most highly oxidized towards the top of bundle, so it is likely that the "flowered" cladding survived in lower regions where the cladding was less heavily oxidized.

(2) Shattering of the fuel was observed in a limited number of tests that were slowly cooled. Limited fragmentation of the CORA-2 bundle was observed, extensive fragmentation was observed in the PBF SFD 1-4 experiment, and extensive fragmentation was observed in the SBU experiments. However, shattering did not occur in the majority of the experiments that were slowly cooled. The only mechanism that appears to be consistent for the experiments where extensive fragmentation occurred was that the peak temperatures were greater than $2300 \mathrm{~K}$ for an extended period of time and the upper portion of the bundle where fragmentation occurred was steam starved.

Therefore, the following general criteria are proposed for rubble formation. First, the cladding will shatter into coarse fragments if the cladding is embrittled and the cladding is quenched, unless the cladding is surrounded by previously molten material. Second, the fuel will shatter if the cladding has been previously removed by shattering or melting in a steam starved environment and the fuel is quenched, unless the fuel is surrounded by previously molten material or reaches a peak temperature near the melting point of $(\mathrm{U}, \mathrm{Zr}) \mathrm{O}_{2}$. The amount of fuel fragmentation will increase from a minimum to complete 
fragmentation as the peak temperature of the fuel increases from the melting point of Zircaloy to a temperature near the melting point of $(\mathrm{U}, \mathrm{Zr}) \mathrm{O}_{2}$. Fuel desintering will only occur if the fuel has limited burnup and is cooled from a peak temperature of between $2200-2400 \mathrm{~K}$ under steam starved conditions. The breakup of the cladding and fuel will only occur on cooldown when the temperatures are relatively low. The specific parameters to be used will be determined through code-to-data comparisons.

The transition criteria for the formation of a metallic cohesive debris bed will remain the same as before. That is, a cohesive bed will be formed with zero open porosity for vertical flow when the frozen crust thickness predicted by the fuel rod relocation models exceeds $5 \mathrm{~cm}$. The vertical flow area region will be linearly decreased from the original flow area to zero as a function of the frozen crust thickness. The corresponding loss coefficient for the reduced flow area will be increased accordingly using a smooth area change formulation. The horizontal flow area computed using the height of the debris within a volume will go to zero as the height of the bed approaches the height of the thermal hydraulic volume. The corresponding loss coefficient will also be based upon a small area change formation. The transition criteria for a ceramic cohesive debris bed will be slightly altered to reflect the experimentally observed trend that the flow area does not instantly reduce to zero across an assembly as ( $\mathrm{U}, \mathrm{Zr}) \mathrm{O}_{2}$ melt temperatures are reached. Instead a linear reduction in flow area, and associated increase in loss coefficient using a smooth area change will be used to reflect a smooth transition to a blocked volume around the melting temperature of $(\mathrm{U}, \mathrm{Zr}) \mathrm{O}_{2}$. The temperature range for the transition will be established during the developmental assessment of the model.

\subsubsection{Debris Bed Characteristics}

The porosity and effective particle size of a rubble debris bed will be modified to reflect the heterogeneous nature of the debris beds observed in the experiments where rubble beds were formed. Because the porosity and particle size are not truly independent, particle sizes and porosities will be assumed to be related. For example, very fine particles form rubble beds of relatively low porosity while particles on the order of the original fuel rod dimensions form a rubble debris bed with approximately the same volume as the original intact bundle. The particle size and porosity will also reflect the variation in fragmentation as noted in the previous section. Therefore, the following general criteria will be used for the initial porosity and effective particle size in rubble beds. The specific values will be refined as additional results became available from the metallographic examination of the CORA and other reflood tests.

1. The average particle sizes for rubble formed during quench (non desintered fuel) will be based on the axially varying bundle average data from CORA-12, CORA-13, CORA-17, LOFT FP-2, and PBF SFD ST. An initial particle size will be based on the visual examination of the cross-sections of the bundles and reflect an average of $1 / 10$ the original fuel pellet diameter for the debris in the coolant channels, (the particles varied from $\sim 1 / 20$ to full pellet diameters) and fuel pellet diameter in the fuel rod stubs. The surface-weighted and volume-weighted average particle sizes will be based upon the fraction of pellet stubs and loose debris at a given axial elevation. In general terms, the loose debris sitting on top of obstructions including grid spacers, ballooned and ruptured fuel rod stubs, or cohesive blockages will be an average of the fuel rod diameter with the number of particles consistent with the total mass of fuel shattered.

2. The average particle size for desintered fuel in the flow channel will be the estimated fuel grain size. The surface- and volume-weighted average particle size will be determined using the same approach as for quench-induced fragmentation including the effect of fuel 
rod stubs.

3. The average open porosity of the rubble in the flow channels will be determined from the same experiments noted in item 1 above but will be correlated to the volume average particle size to reflect the variation in porosity for debris within an array of fuel rod stubs and for debris where the fuel rods have been completely shattered. Because porosity data are not available for the ESBU experiments where fuel desintering occurred, the porosity will be assumed to be zero. In all cases, a fluidization limit will have to be determined for the loose debris above open obstructions, such as grid spacers, to insure that porosity and bed height will increase to the appropriate levels when high velocity vapor or liquid is injected from below.

The porosity of cohesive debris beds will be modified to reflect the foaming of metallic debris beds in the presence of control and structural materials and the porosity observed in ceramic blockage. It is likely that the porosity of the metallic and ceramic material will be a strong function of pressure (less foaming at high pressure) and fission product retention, however, it is not clear whether there will be adequate data to quantify the functional dependence of such effects. For the time being, the porosity of metallic blockages will be linear functions of (a) control and structural mass fractions and (b) pressure with zero closed porosity for a zero control and structure mass fractions (CORA-2 and ESBU) and 50\% porosity at atmospheric pressure for a well mixed composition (CORA-5 and DF-4) and zero porosity for high pressures (SFD 1-4). The porosity of ceramic blockages will be determined from the porosity of ceramic blockages in the PBF SFD, LOFT LP-FP-2 experiments and would include the influence of pressure and irradiation history.

The surface- and volume-averages particle sizes in rubble beds and porosities in all debris beds would also be modified to account for the melting of the debris. The porosity would be reduced as the composition mass-weighted melting temperature are reached using the temperatures shown on Figure 232. That is, for a bed composed primarily $\mathrm{UO}_{2}$ and $\mathrm{ZrO}_{2}$, the porosity would be reduced linearly to zero as $(\mathrm{U}, \mathrm{Zr}) \mathrm{O}_{2}$ melting temperature are reached. If the debris bed is composed of $\mathrm{UO}_{2}, \mathrm{ZrO}_{2}, \mathrm{Zircaloy}$, control material, and structural material, then the porosity would be reduced linearly from the original porosity starting at formation temperatures where Zircaloy and control/structural material eutectics are noticeable $(\sim 1500 \mathrm{~K})$ and decreasing to zero as $(\mathrm{U}, \mathrm{Zr}) \mathrm{O}_{2}$ melting temperatures are reached. For cohesive debris beds, the height of the bed would only be reduced as the upper melting point is reached, that is, when the porosity approaches zero. For rubble debris beds, the height of the bed would be reduced as the porosity is decreased assuming that rubble settles to eliminate any voided regions. The average particle size in the rubble debris would also be decreased to be consistent with the particle size/porosity relationship noted in the preceeding section. The effects of fission gas release or vapor pressure in the more volatile constituents, leading to additional foaming as above the melting point is reached would be neglected. The radial spreading of material from one debris bed region or thermal hydraulic flow channel to another, would also be neglected until a molten pool has been formed. 


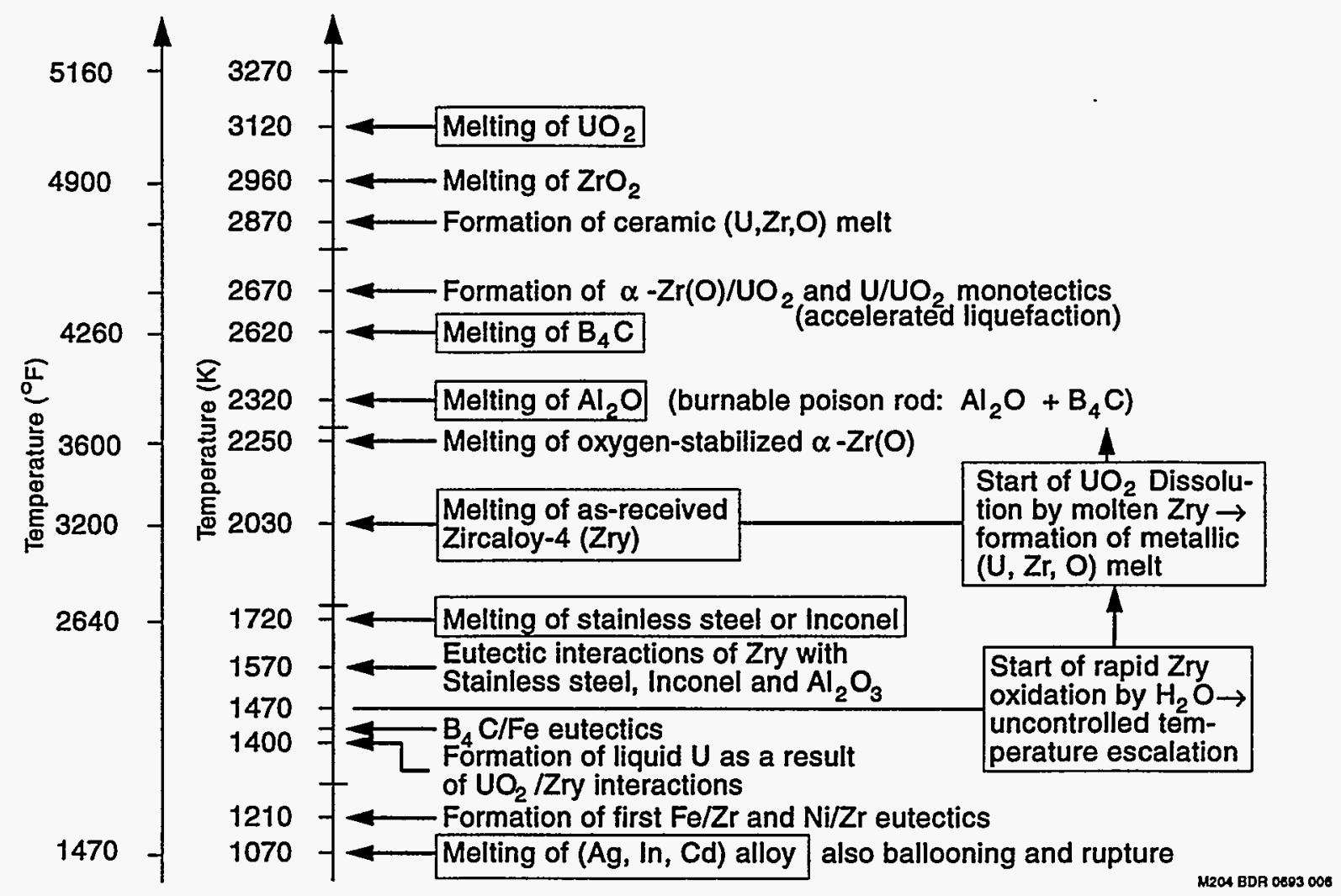

Figure 2-32. Important damage progression stages and melting temperatures.

\subsubsection{Effective thermal conductivity models for lower plenum loose debris}

Reference 2-23 compares recently measured effective thermal conductivities with various correlations for predicting the effective conductivity of a porous debris. In the Reference 2-23 tests, three different packing materials (alumina, aluminum, and glass) of various particle diameters (2.5 to $13.5 \mathrm{~mm}$ ) were tested. Internal bed temperature profiles and corresponding effective thermal conductivities were measured under steady state conditions for temperatures ranging from 350 to $1300 \mathrm{~K}$. This recent study concluded that the overall effective conductivity could best be modeled by applying the following correlation

$\mathrm{k}_{\text {eff }}=0.8 \mathrm{k}_{\text {cond }-\mathrm{ZBS}}+4 \varepsilon \mathrm{d}_{\mathrm{p}} \sigma \mathrm{T}^{3}$

where $\mathrm{k}_{\text {cond-zBS }}$ is the effective conductivity due to conduction proposed by Zehner-Bauer-Schlünder, which is given by $2-24,2-25$ 
$k_{\text {cond -zBS }}=k_{f}\left[(1-\sqrt{1-\phi})\left(1+\phi \frac{k_{r}}{k_{f}}\right)+\sqrt{1-\phi}\left[\alpha \cdot \frac{k_{s}^{\prime}}{k_{f}}+(1-\alpha) \frac{k_{s o}}{k_{f}}\right]\right]$

$\frac{k_{s o}}{k_{f}}=\frac{2}{N}\left[\frac{B\left(\frac{k_{s}^{\prime}}{k_{f}}+\frac{k_{r}}{k_{f}}-1\right) \frac{k_{f}^{\prime}}{k_{s}^{\prime}}}{N^{2}} \ln \left(\frac{\left(\frac{k_{s}^{\prime}}{k_{f}}+\frac{k_{r}}{k_{f}}\right)}{B}\right)-\frac{B-1}{N}+\left(\frac{k_{f}}{k_{f}}-B\right) \frac{B+1}{2 B}\right]$

$N=\left[1+\left(\frac{k_{r}}{k_{f}}-B\right) /\left(\frac{k_{s}^{\prime}}{k_{f}}\right)\right]$

$k_{s}^{\prime}=k_{s}\left(\frac{1}{1+B_{\text {iox }}}\right)$

$B_{i o x}=\left(\frac{S_{o x}}{k_{o x}}\right) \frac{k_{s}}{d_{p}}$

$k_{r}=4 \sigma\left(\frac{\varepsilon}{2-\varepsilon}\right) x_{R} T^{3}$

$\mathrm{B}=\mathrm{C}_{\mathrm{form}}\left(\frac{1-\phi}{\phi}\right)^{10 / 9}$

$\alpha=\frac{23 \rho_{k}^{2}}{1+22 \rho^{4 / 3} k}$

where

$\begin{array}{lll}\mathrm{C}_{\mathrm{form}} & = & \text { particle shape factor } \\ \mathrm{x}_{\mathrm{R}} & = & \text { radiation effects factor } \\ \rho_{\mathrm{k}}^{2} & = & \text { contact conduction factor } \\ \frac{\mathrm{S}_{\mathrm{ox}}}{\mathrm{k}_{\mathrm{ox}}} & = & \text { oxidation effects factor. }\end{array}$

Reference 2-25 provides typical values for the above particle characteristics for many of the particle materials, shapes, and sizes of interest in severe accident debris beds. Values for the form factor for spherical and broken particles are summarized below in Table 2-2. Expressions for estimating the radiation 
effects factor, $x_{R}$, as a function of the radiation influence coefficient, $R_{\text {form }}$, and the mass fraction, $M F$, are also included in Table $2-2$.

Table 2-2. Typical parameters and equations for estimating form and radiation effects factors.

\begin{tabular}{|c|c|c|c|}
\hline $\begin{array}{c}\text { Particle } \\
\text { geometry }\end{array}$ & $\begin{array}{c}\text { Form } \\
\text { factor, } \\
\mathrm{C}_{\mathrm{form}}\end{array}$ & Radiation effects factor, $\mathrm{x}_{\mathrm{R}}$ & $\begin{array}{c}\text { Radiation } \\
\text { influence } \\
\text { coefficient, } \\
\mathrm{R}_{\mathrm{form}}\end{array}$ \\
\hline $\begin{array}{c}\text { spherical - } \\
\text { monodisperse }\end{array}$ & 1.25 & $\mathrm{x}_{\mathrm{R}}=\mathrm{R}_{\mathrm{form}} \mathrm{d}_{\mathrm{p}}$ & 1.00 \\
\hline $\begin{array}{c}\text { spherical - } \\
\text { binary mixture }\end{array}$ & 1.25 & $\mathrm{x}_{\mathrm{R}}=\frac{1}{\left(\mathrm{M} \mathrm{F}_{\mathrm{p}}\right) /\left(\mathrm{R}_{\mathrm{form}} \mathrm{d}_{\mathrm{p} 1}\right)+\left(1-\mathrm{MF}_{\mathrm{f}}\right) /\left(\mathrm{R}_{\mathrm{form}} \mathrm{d}_{\mathrm{p} 2}\right)}$ & 1.00 \\
\hline broken & 1.4 & $\mathrm{x}_{\mathrm{R}}=\frac{1}{\sum_{i=1.0}\left(\mathrm{MF}_{\mathrm{i}}\right) /\left(\mathrm{R}_{\mathrm{form}} \mathrm{d}_{\mathrm{pi}}\right)}$ & 1.00 \\
\hline
\end{tabular}

Values for the oxidation effects factor and the contact conduction factor and must be determined experimentally. Typical values for these factors and emissivities for particles considered in severe accident debris beds are listed below in Table 2-3. $2-25,2-26,2-27$

Table 2-3. Typical particle characteristics.

\begin{tabular}{|c|c|c|c|}
\hline Material & Emissivity & $\begin{array}{c}\text { Oxidation Effects Factor, } \\
\mathrm{S}_{\mathrm{ox}} / \mathrm{k}_{\mathrm{ox}}\end{array}$ & $\begin{array}{c}\text { Contact Conduction } \\
\text { Factor, } \mathrm{o}_{\mathrm{k}}{ }_{\mathrm{k}}\end{array}$ \\
\hline \hline Ceramic & $0.8-0.85$ & 0.00 & $3.5 \times 10^{-4}$ \\
\hline Metallic & & & \\
Oxidized & 0.85 & $0.15 \times 10^{-3}$ & $1.5 \times 10^{-4}$ \\
Unoxidized & 0.30 & 0.00 & $1.5 \times 10^{-4}$ \\
\hline
\end{tabular}

Results in Reference 2-23 indicate that there is good agreement between data and predictions obtained with Equation (2-12) for a range of particle sizes, particle compositions, and particle temperatures. However, it should be noted that a limited number of comparisons between results from Equation (2-12) and experimental data have been performed. For example, the Reference 2-23 study was limited because all of the particle beds consisted of a single particle size (although a range of particle sizes were tested), all the particle beds had approximately the same bulk porosity $(0.38)$, all of the tests were conducted at the same pressure, and none of the particles were oxidized. Hence, it is recommended that a revised model based on Equation (2-12) be incorporated into the code and then assessed for a wider range of conditions (see Sections 2.4 and 5). If the assessment indicates that this model more accurately predicts the heat transfer in debris beds than the existing model, then the existing model could be replaced. 


\subsection{Expected Impact of Improved Models}

The most noticeable impact of the new models will be a much more accurate representation of the formation and behavior of debris beds relative to the currently available data base. Cohesive debris beds will form more gradually over a temperature range more consistent with (a) the range of melting temperatures of different mixture of materials and (b) the rod-to-rod variations in power and cooling conditions in a representative assembly. Rubble debris beds will form over a range of peak temperatures and cooling rates as shown in both the quenched and slow-cooled experiments. More importantly, rubble debris beds will not always be assumed to be spatially uniform. In most cases, as discussed in Section 2.1, the relative fraction of the fuel that is shattered during quench will still be small relative to the overall height of the bundle. Thus the previous assumption that all of the fuel would shatter during the quenching process where the cladding was embrittled clearly overpredicts the volume of rubble debris beds. The desintering of the fuel, although it occurs over a very limited range of conditions, will also be very important because a very dense layer of debris can be formed. This layer will not be coolable from water from above as indicated by the particle size dependencies of most dryout correlations. If the debris forms on top of a cohesive layer, the debris will act much like an additional layer of cohesive debris. Fuel desintering during cooldown will also have an important impact on fission gas release because all of the fission gases on the grain boundaries will be released.

These modeling changes will also have a noticeable impact on plant calculations, and code-to-data comparisons for experiments such as LOFT LP-FP-2, because the flow diversion associated with the formation of debris beds will occur more gradually over a range of temperatures rather than at a fixed temperature as in the current models. For example, as a metallic layer is formed, the reduction in vertical flow area and increase in local vertical loss coefficients will occur at lower temperatures due to the foaming of the melt in the presence of control and structural materials. The reduction in flow areas, both vertical and horizontal, in a region of ceramic melting will also occur more gradually. Although the specific effect of flow diversions can not be quantified until the developmental assessment of these proposed models is completed, the effect should be very beneficial in several instances. For example, as noted earlier, in the LOFT LP-FP-2 experiment, a radial nodalization significantly finer than typically used in plant calculations was necessary to properly account for the observed flow diversion. With these modeling changes, a radial nodalization more typical of plant calculations should be able to better predict the observed behavior. A number of plant calculations have been performed where the formation of blockages or rubble debris has been very sensitive to nodalization because of the abrupt transition from one geometry to another. These proposed modeling changes should significantly reduce the sensitivity to nodalization during the later stages of an accident. Recent calculations for TMI-2 have indicated that a number of problems associated with the current transition models could be eliminated by the proposed changes. For example, outer assemblies in the core may have heated up too slowly due to the abrupt diversion of steam from the center of the core to the outer assemblies. In addition, the flow moving back toward the center of the core above the blocked region may have been too high resulting in an over prediction of the temperature of the centermost portion of the upper grid. (A horse shoe damage pattern was formed so that the peak temperature of the upper grid had a maximum at a mid-radius position rather than in the radial center).

The proposed changes in debris bed characteristics models will have a significant impact on the subsequent heating and melting of debris beds. The incorporation of more detailed thermal-hydraulic models will be much more influenced by the local rubble bed porosities and particle sizes than the previous 
thermal hydraulic models during the dryout and quenching of debris beds. The convective heat transfer and flow patterns in single phase steam will also be impacted since debris-bed-specific friction factors and heat transfer coefficients will be used rather than existing RELAP5 bundle correlations. However, the quantification of these effects will require assessment using the new thermal-hydraulic models. As discussed in the next section, the effective heat transfer within debris beds will also depend strongly on the porosity and effective particle size, independent of the specific correlations ultimately selected.

\subsubsection{Comparison of Effective Thermal Conductivity Models}

As discussed in Section 2.3.3, the overall effective conductivity of a porous debris bed is estimated in SCDAP/RELAP5 using the Imura-Takegoshi and Vortmeyer model. Figure 2-33 illustrates the manner in which this model predicts the total effective conductivity as a function of particle temperature for a debris bed containing ceramic $0.01 \mathrm{~m}$ diameter particles with $50 \%$ porosity. Contributions associated with conduction and radiation to the overall Imura-Takegoshi and Vortmeyer model effective conductivity are also shown in Figure 2-33. As indicated in this figure, radiation contributions exceed the conduction contributions once the particle temperatures exceed approximately $1420 \mathrm{~K}$.

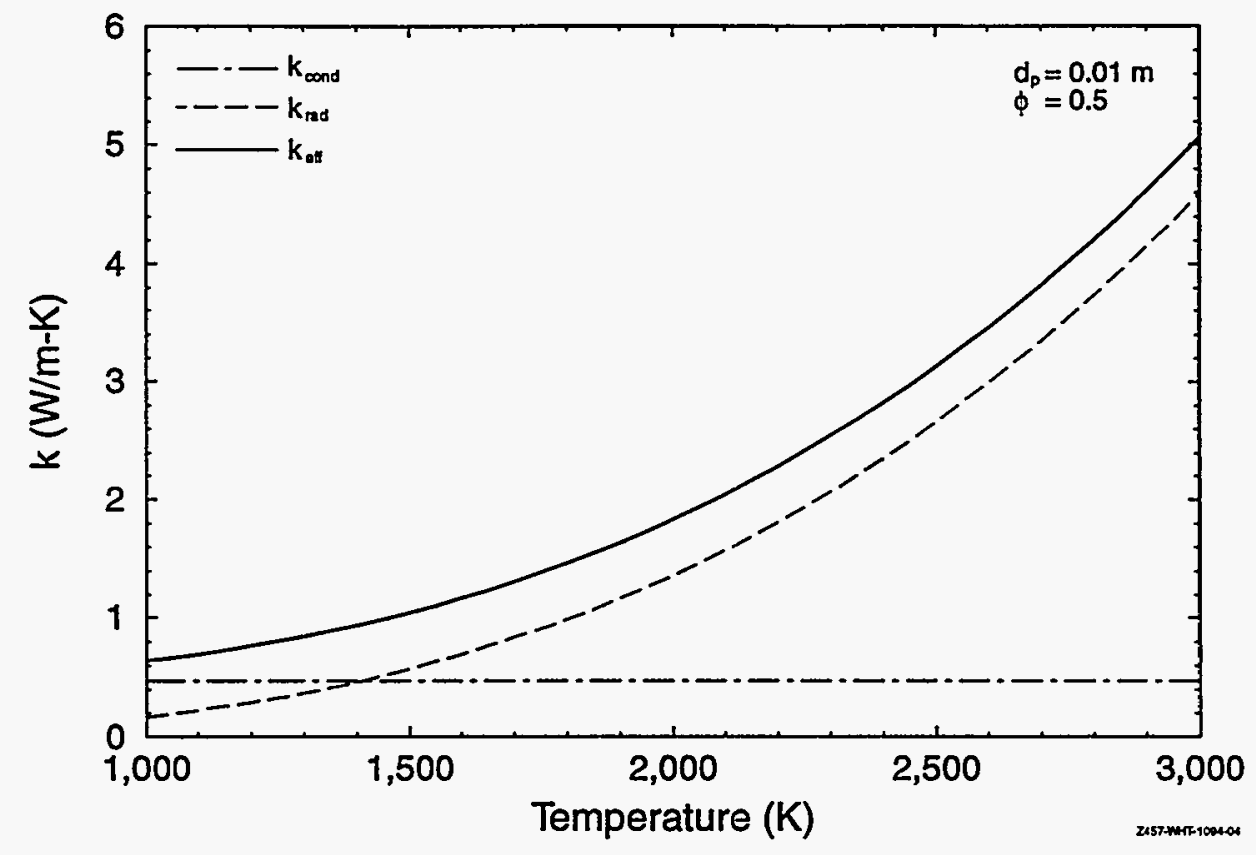

Figure 2-33. Effective conductivity predicted by the INEL Imura-Takegoshi and Vortmeyer model.

Figures 2-34 and 2-35 illustrate the sensitivity of Imura-Takegoshi and Vortmeyer model effective conductivity predictions to particle size and porosity. Upper and lower bounds for these sensitivity studies correspond to upper and lower ranges expected for debris bed particle size and porosity during a severe accident. Results in Figure 2-34 were obtained from calculations assuming a ceramic debris bed with 50\% porosity. As illustrated in this figure, higher effective conductivities are predicted for debris beds containing larger particles. This increase in effective conductivity comes from the radiation heat transfer 
contribution to the effective conductivity and is associated with the increased surface area for radiation associated with larger particles. Results in Figure 2-35 were obtained from calculations assuming a ceramic debris bed containing $0.01 \mathrm{~m}$ diameter particles. Although not as sensitive to porosity assumptions as to debris bed particle assumptions, results in this figure indicate that higher effective conductivities are predicted for debris beds with lower porosities. This increase in effective conductivity comes from the conduction heat transfer contribution to the effective conductivity and is associated with the reduced volume of solid in the debris bed.

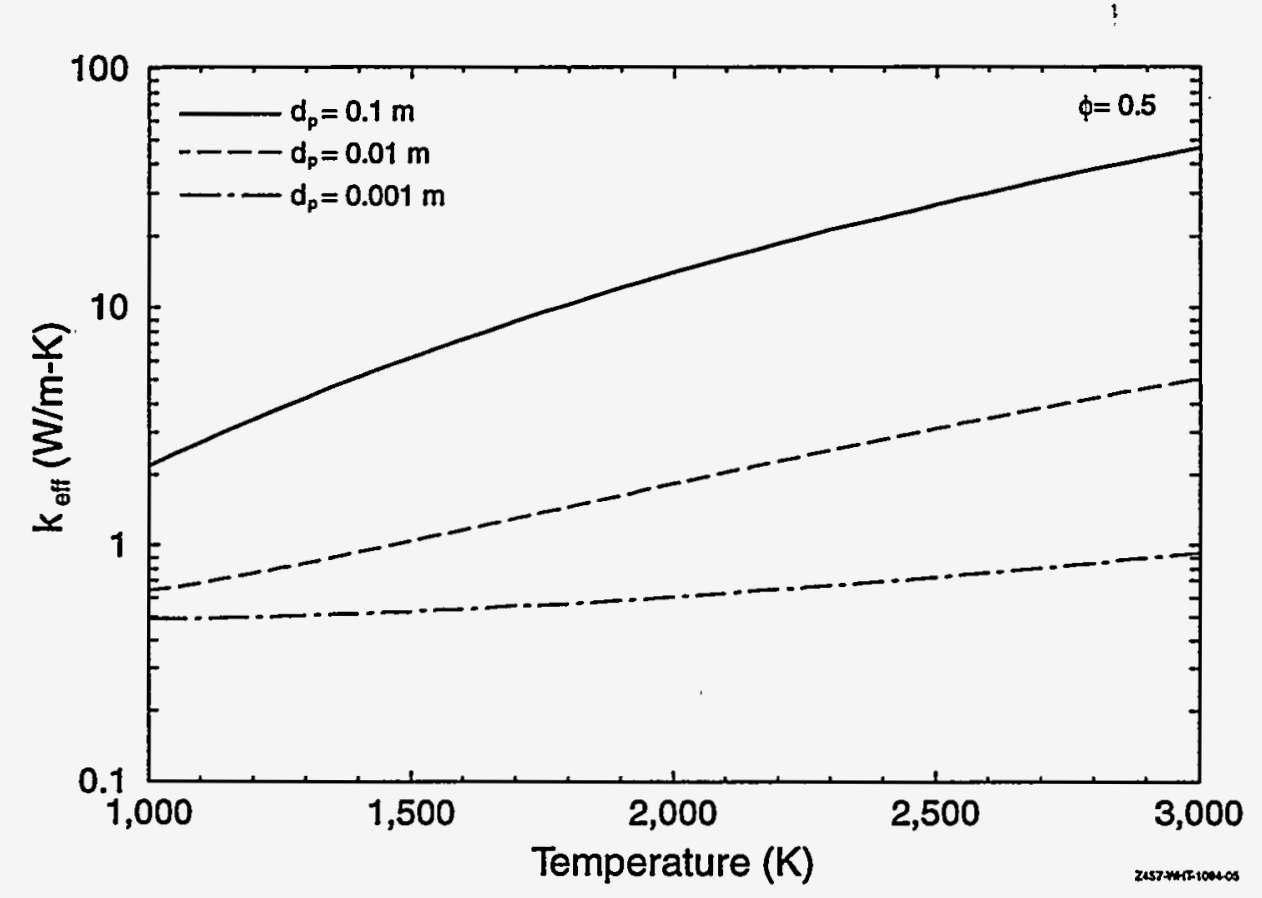

Figure 2-34. INEL Imura-Takegoshi and Vortmeyer model sensitivity to particle diameter. 


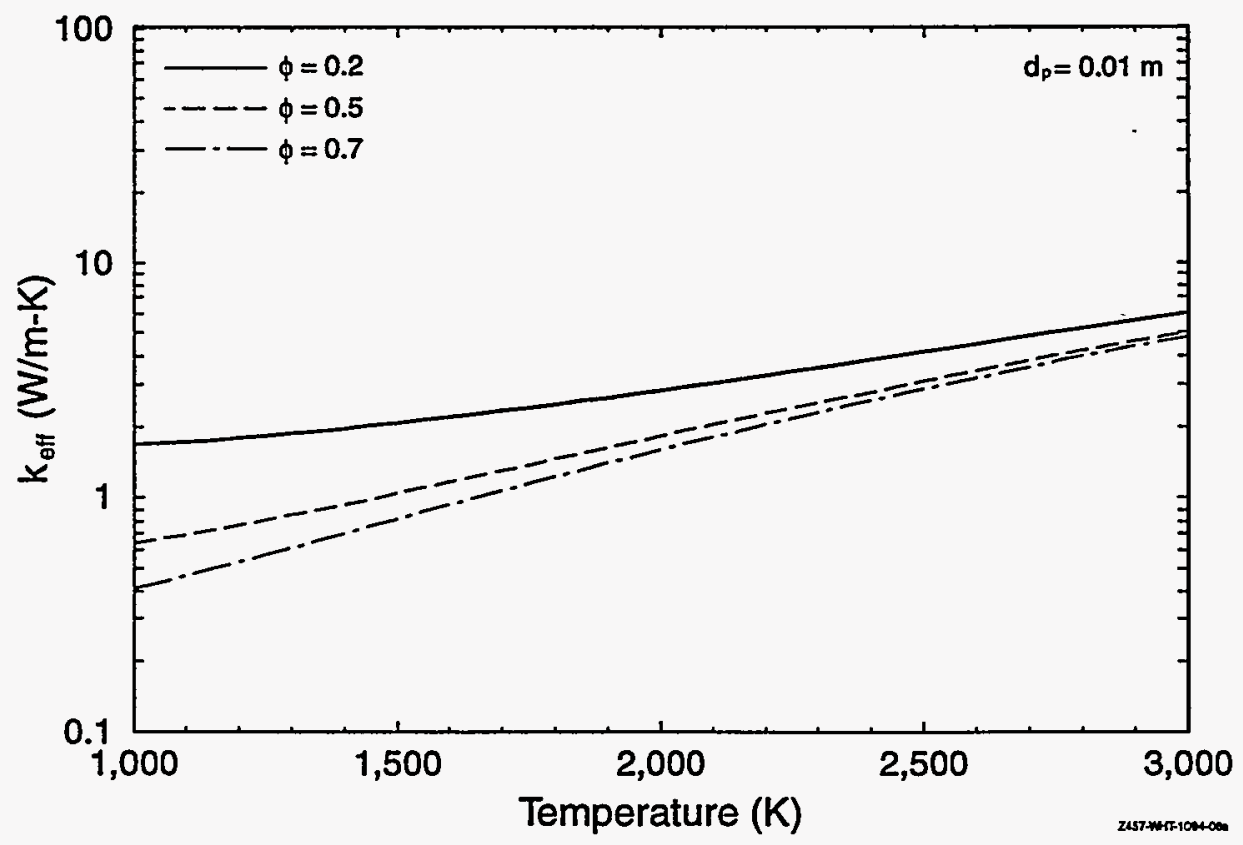

Figure 2-35. INEL Imura-Takegoshi and Vortmeyer model sensitivity to debris bed porosity.

As discussed in Section 2.3.3, the INEL interpretation of the Imura-Takegoshi and Vortmeyer model differs from the SNL interpretation in the DEBRIS model. This difference is attributed to the manner in which the conductivity associated with radiation heat transfer is modeled, specifically in the radiation exchange factor. Figure 2-36 compares predictions from the INEL and SNL interpretation of the ImuraTakegoshi and Vortmeyer model for a debris bed containing $0.01 \mathrm{~m}$ particles with $50 \%$ porosity. As illustrated in Figure 2-36, the effective conductivity predicted by the SNL version of this model is considerably higher than the INEL version of this model. 


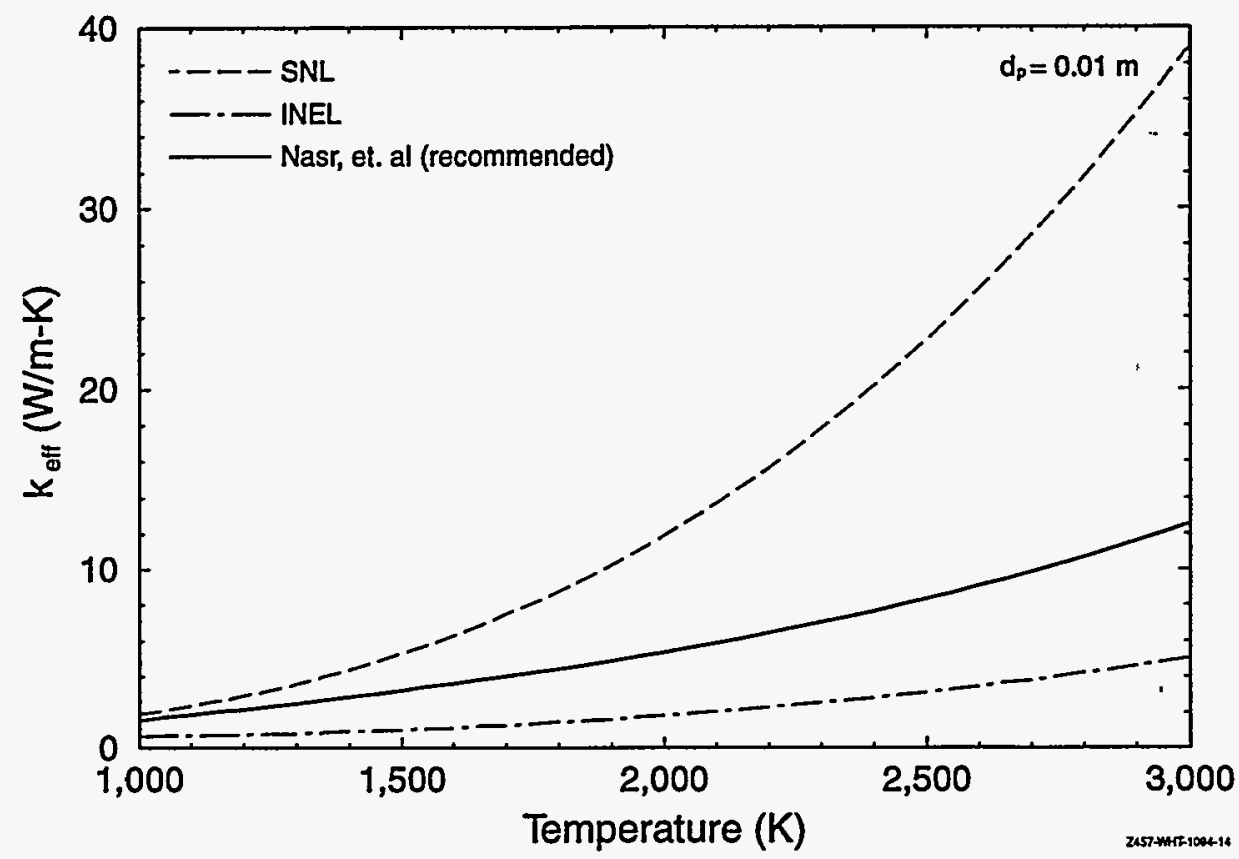

Figure 2-36. Comparison of various effective conductivity model predictions.

In Section 2.3.3, it is recommended that the Nasr et al. model for predicting debris bed effective conductivity be incorporated in SCDAP/RELAP5. This correlation was developed using data from a wide range of particle sizes, materials, and temperatures. Figure 2-36 also contains a curve representing predictions from the Nasr, et al. model. As illustrated in Figure 2-36, the Nasr, et al. correlation will lead to effective conductivities that are between a factor of 2 to 6 times larger than values that are predicted with the existing SCDAP/RELAP5 effective conductivity model. However, the proposed revision will still yield values that are lower than the SNL interpretation of the Imura-Takegoshi and Vortmeyer model.

Figures 2-37 through 2-39 illustrate the sensitivity of the Nasr, et al. model to particle size, debris bed porosity, and particle composition, respectively. As discussed below, the Nasr, et al. model sensitivity to particle size is similar to the Imura-Takegoshi and Vortmeyer model currently in SCDAP/RELAP5. However, the sensitivity of the Nasr, et al. model to debris bed porosity differs considerably from the Imura-Takegoshi and Vortmeyer. 


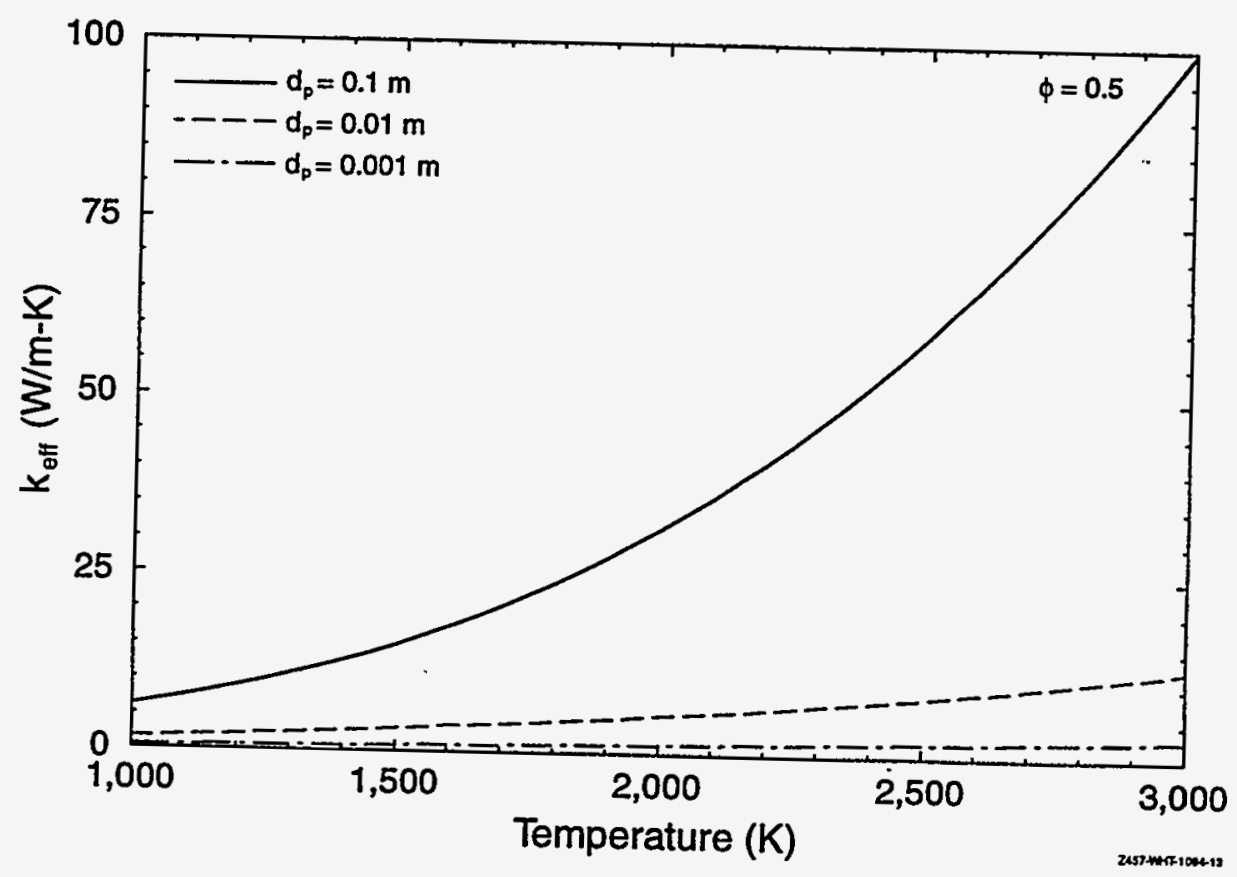

Figure 2-37. Sensitivity of Nasr, et al. model predictions to particle diameter.

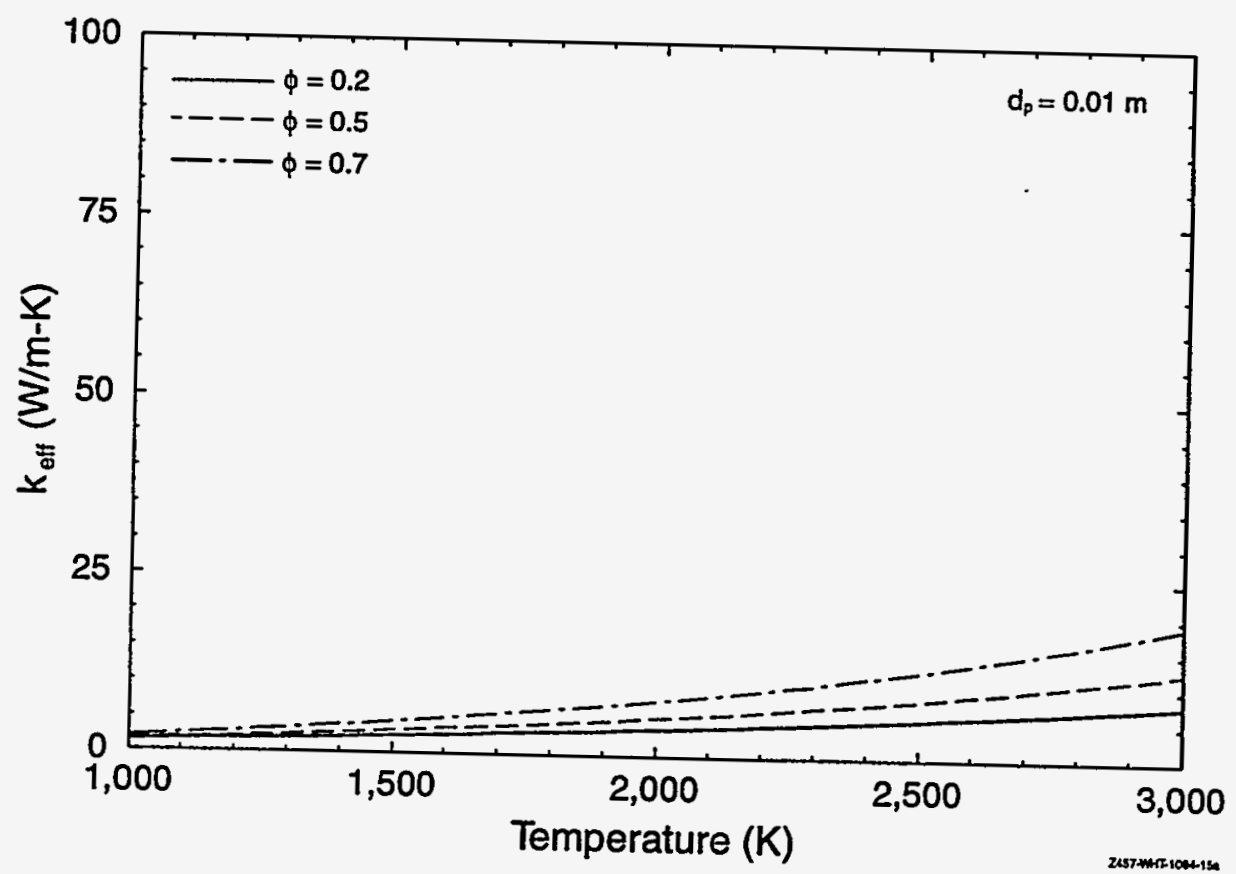

Figure 2-38. Sensitivity of Nasr, et al. model predictions to debris bed porosity. 


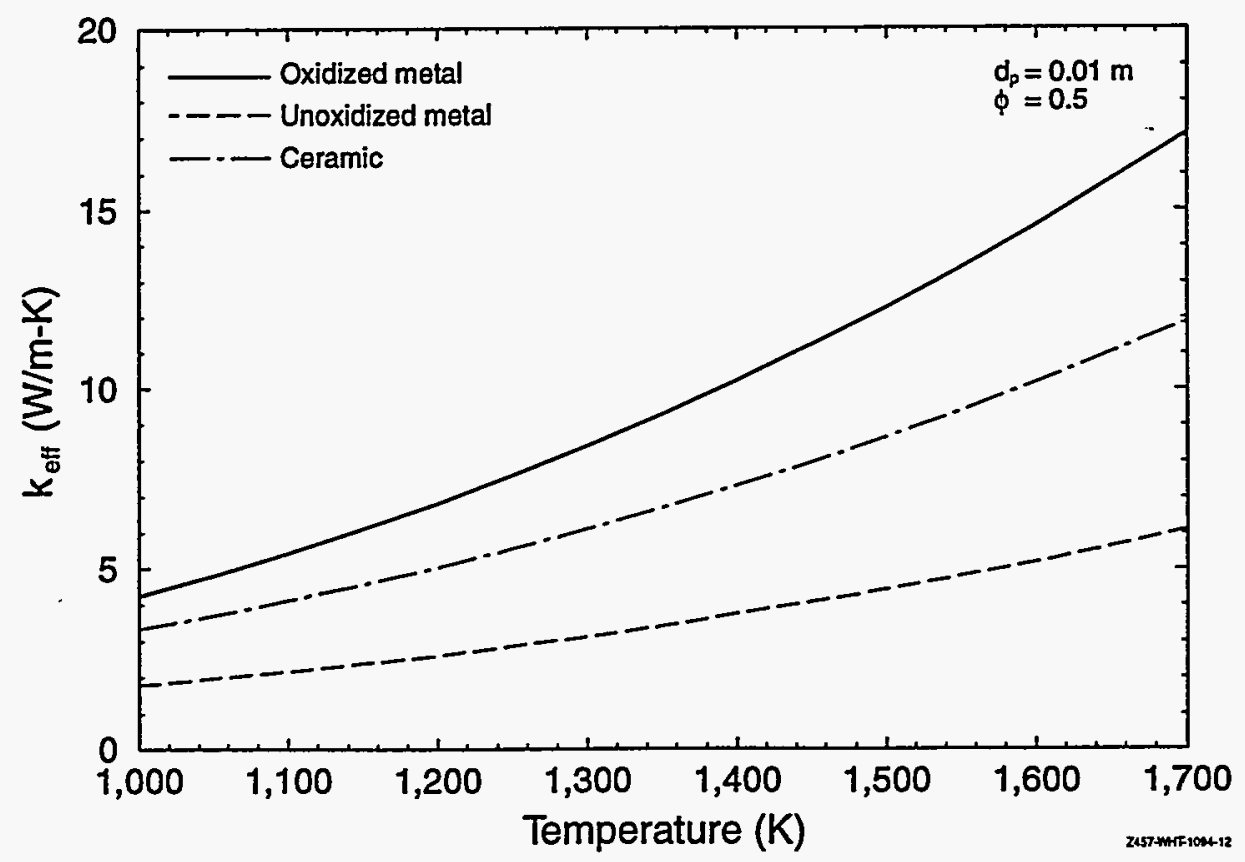

Figure 2-39. Sensitivity of Nasr, et al. model predictions to particle composition.

In Figure 2-37, calculation results are compared for cases assuming a debris bed containing uniformly sized, spherical ceramic debris particles with $50 \%$ porosity. Consistent with the INEL ImuraTakegoshi and Vortmeyer model results shown in Figure 2-34, higher effective conductivities are predicted by the Nasr, et al. model for cases assuming larger particle sizes in the debris bed.

Figure 2-38 compares results from calculations assuming a debris bed containing uniformly sized, spherically shaped, $0.01 \mathrm{~m}$ diameter ceramic debris particle debris beds with various porosities. Unlike the INEL Imura-Takegoshi and Vortmeyer model results shown in Figure 2-35, higher effective conductivities are predicted by the Nasr, et al. model for cases assuming larger porosity debris beds. As discussed in Section 5 , additional work is needed to assess the Nasr, et al. model's sensitivity to debris bed porosity.

Although the current INEL Imura-Takegoshi and Vortmeyer model is capable of considering various particle compositions, the current SCDAP/RELAP5 model does not consider oxidation effects. In Figure 2-39, calculation results are presented for cases considering various particle material compositions and oxidations. To obtain the results shown in Figure 2-39, calculations considered a debris bed containing uniformly sized, spherical, $0.01 \mathrm{~m}$ diameter particles with $50 \%$ porosity. Results shown in Figure 2-39 indicate that the effective conductivities predicted for oxidized metallic debris will exceed the effective conductivities predicted for either ceramic or unoxidized metallic debris. This is because oxidized metallic debris has a high emissivity and a high oxidation effects factor (see Table 2-3). As discussed in Section 2.3.3, the Nasr, et al study did not assess their proposed model against tests considering particles composed of materials expected in a severe accident debris bed. Hence, it is proposed that additional work be performed to assess the Nasr, et al. model against tests considering more prototypic severe accident debris bed material compositions. 


\subsection{References}

2-1. J. Broughton, et al., "A Scenario of the Three Mile Island Unit 2 Accident," Nuclear Technology, 87, August 1989, pp. 34-53.

2-2. J. Hohorst, et al., TMI-2 Analysis Using SCDAP/RELAP5/MOD3.1, INEL-94-0157, November 1994.

2-3. S. Hagen, et al., Interactions in Zry/UO2 Fuel Rod Bundles with Inconel Spacers at Temperatures above 1200 C; (Posttest Results of Several Fuel Damage Experiments CORA-2 and CORA-3), Kernforschungszentrum, Karlsruhe Report, KfK 4378, 1990.

2-4. S. Hagen, Kernforschungszentrum, Karlsruhe, Personal Communication.

2-5. S. Hagen, et al., Temperature Escalation in PWR Fuel Rod Simulator Bundles due to the Zircaloy/ Steam Reaction: Test ESBU-2A Test Results Report, KfK 3509, July 1984.

2-6. S. Jensen, D. Akers, B. Pregger, Postirradiation Examination Data and Analyses for OECD LOFT Fission Product Experiment LP-FP-2, OECD LOFT-3810, Vol. I, December 1989.

2-7. A. D. Knipe, et al., PBF Severe Fuel Damage Scoping Test - Test Results Report, NUREG/CR4683, EGG-2413, August 1986.

2-8. Z. R. Martinson, et al., PBF Severe Fuel Damage Test 1-1 Test Results Report, NUREG/CR-4684, EGG-2463, October 1986.

2-9. D. A. Petti, et al., PBF Severe Fuel Damage Test 1-4 Test Results Report, NUREG/CR-5163, EGG-2542, April 1989.

2-10. Z. R. Martinson, et al., PBF Severe Fuel Damage Test 1-3 Test Results Report, NUREG/CR-5354, EGG-2565, October 1989.

2-11. R. Gauntt, et al., The DF-4 Fuel Damage Experiment in ACRR with a BWR Control Blade and Channel Box, NUREG/CR-4671, SAND86-1443, November 1989.

2-12. R. Gauntt, Sandia National Laboratories, Personal Communications.

2-13. C. M. Allison, et al., SCDAP/RELAP5/MOD3.1 Code Manual, Volume II: Damage Progression Model Theory, NUREG/CR-6150, EGG-2720, October 1993.

2-14. S. Imura and E. Takegoshi, "Effect of Gas Pressure on the Effective Thermal Conductivity of Packed Beds," Heat Transfer Japanese Research, Vol. 3, No. 4, p. 13, 1974.

2-15. D. Vortmeyer, "Radiation in Packed Solids," Proceedings from the 6th International Heat Transfer Conference, Toronto, Canada, Vol. 6, pp. 525-539, 1978.

2-16. W. B. Argo and J. M. Smith, Chem. Engng. Progr., Vol. 49, p. 443, 1953.

2-17. W. Schotte, A.I.Ch.E.J. Vol. 6, p. 63, 1960.

2-18. M. J. Laubitz, Canadian. J. Physics, Vol. 37, p. 798, 1959.

2-19. N. Wakao, and K. Kato, J. of Chem. Engng. Japan, Vol. 2, p. 24, 1969.

2-20. G. Kasparek and D. Vortmeyer, Wärme- und Stoffüberttragung, Vol. 9, p. 117, 1976; Dissertation, Techn. Universität Müchen, 1975.

2-21. R. C, Schmidt and R. D. Gasser, Models and Correlations of the DEBRIS Late-Phase Melt Progression Model, SAND93-3922, December 22, 1993. 
2-22. C. M. Allison, et al., SCDAP/RELAP5/MOD3.1 Code Manual, Volume V: Developmental Assessment, NUREG/CR-6150, EGG-2760, December 1993.

2-23. K. Nasr, R. Viskanta, and S. Ramadhyani, "An Experimental Evaluation of the Effective Thermal Conductivities of Packed Beds at High Temperatures," to be published in the ASME Journal of Heat Transfer.

2-24. P. Zehner and E. U. Schlünder, "Thermal Conductivity of Granular Materials at Moderate Temperatures (in German)," Chemie Ingenieur Technik, Vol. 42, pp. 933-941.

2-25. R. Bauer and E. U. Schlünder, "Effective Radial Thermal Conductivity of Packing in Gas Flow. Part II. Thermal Conductivity of the Packing Fraction without Gas Flow," International Chemical Engineering, Vol. 18, No. 2, pp. 189-204.

2-26. C. M. Allison, et al., SCDAP/RELAP5/MOD3.1 Code Manual, Volume IV: MATPRO - A Library of Materials Properties for Light-Water-Reactor Accident Analysis, NUREG/CR-6150, EGG-2720, November 1990.

2-27. P. Zehner, Dissertation, University of Karlsruhe (TH), 1972. 
$2-46$ 


\section{MOLTEN POOL FORMATION AND GROWTH}

During a severe accident, there is the potential for molten pools to form that are internally heated by fission product decay heat. Two locations within the reactor vessel have been identified where such pools may form - the core region and the lower plenum of the reactor vessel after relocation of molten material from the core region. Because heat transfer from molten pools may significantly impact subsequent accident progression, a study was conducted to review existing experimental and analytical studies of molten pool behavior, assess uncertainties in the current SCDAP/RELAP5/MOD3.1 $1^{3-1}$ molten pool heat transfer model, and make recommendations for reducing uncertainties in the SCDAP/RELAP5/MOD3.1 molten pool heat transfer model.

\subsection{Molten Pool Formation}

The first occurrence of the melting of fuel and cladding oxide marks a significant advancement in the severity of an accident. If melting is driven by decay heat, rather than oxidation, melt progression will continue unless reflood occurs. The molten material does not immediately slump from the core region. Instead, the liquefied material either permeates into a colder region of the core and freezes or the movement of the liquefied material is blocked by the nonporous debris formed during the metallic meltdown of the core. As liquefaction continues, the possibility increases that the pool may melt the material supporting it, and a large amount of molten material may suddenly slump into the lower head of the reactor vessel. Thus, unabated fuel melting can lead to a thermal attack of the reactor vessel and a significant increase in the severity of an accident.

Figure 3-1 illustrates a partially molten reactor, nodalized into four axial nodes and three axial flow channels. The material in axial nodes 2 and 3 of flow channel 1 has been heated to the point where it is molten. If the region around the molten material is significantly cooler than the freezing temperature of the molten material, then a stable crust of previously molten material is formed that holds the molten material in place. If the region around the molten material is hot, then a stable crust does not form and the force of gravity causes the molten material to spread downward and laterally:

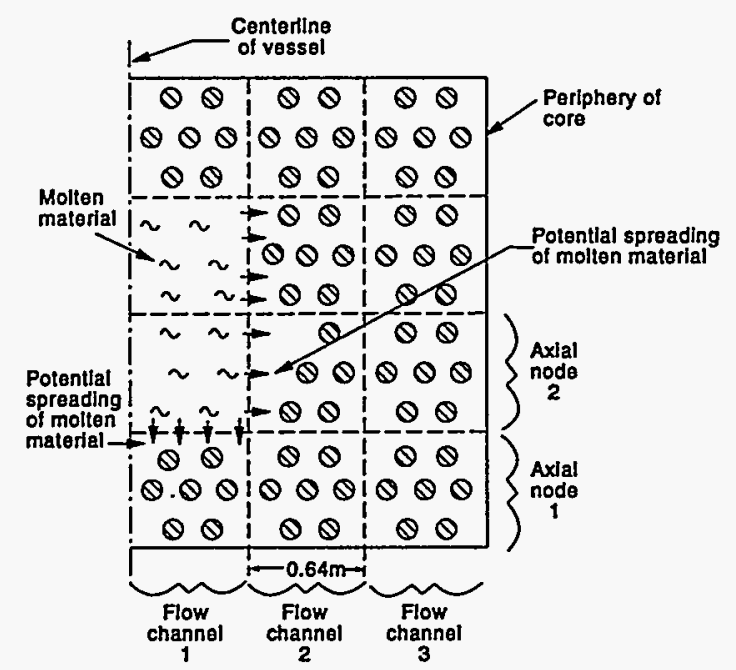

Figure 3-1. Molten pool formation in the reactor core. 
Physical processes associated with molten pool formation and heat transfer in the core and lower head region are illustrated in Figure 3-2. Turbulent natural convection mixes molten material in the pool. Heat fluxes from the molten pool to the crust vary with location. As discussed in Section 3.2.4, the heat flux on the upper part of the crust is significantly larger than that on the bottom part. In the core region, the stability of the crust containing the molten pool is determined based on the relative values of the heat fluxes on its inner and outer surfaces. If the crust is stable, the molten material stays in place. Otherwise, the molten material spreads. In the lower head, a pool will grow if the heat transferred to the crust, via turbulent convection, is greater than the heat removed from the crust to the overlying layer of coolant and to the vessel. The temperature of the molten material is calculated based on the difference between the heat generated in the molten pool and the heat transferred from the molten pool at its boundary.

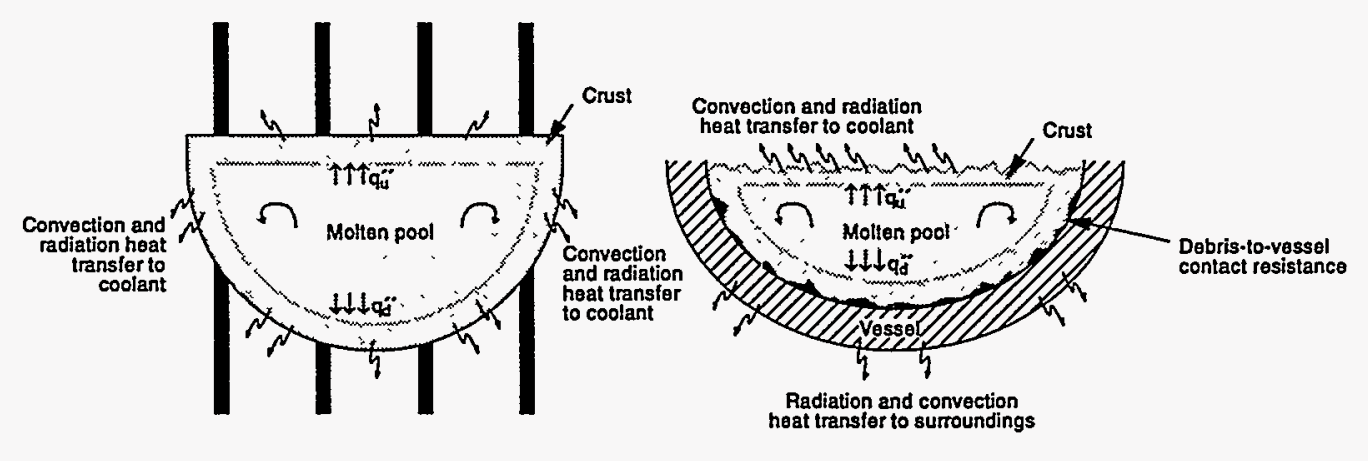

a) Core region

b) Lower head

2us m-100402

Figure 3-2. Heat transfer mechanisms associated with molten pools formation.

\subsection{Review of Existing In-Core and Lower Plenum Debris Models}

Some background material applicable to predicting molten pool heat transfer is summarized in this section. Dimensionless parameters used to characterize molten pools are quantified using results from a representative SCDAP/RELAP5/MOD3.1 calculation. An overview of data applicable to this phenomena is also provided in this section. More detailed information on these subjects may be found in Appendix A.

\subsubsection{Characteristic Dimensionless Groups}

For steady-state heat transfer, data are usually correlated in terms of the surface-averaged Nusselt number, $\mathrm{Nu}$, a modified Rayleigh number, $\mathrm{Ra}$, and the fluid Prandtl number, $\mathrm{Pr}$, which are defined by

$\mathrm{Nu}=\frac{\mathrm{hL}}{\mathrm{k}}$ 
$\mathrm{Ra}=\frac{\mathrm{g} \beta \mathrm{q}^{\prime \prime \prime} \mathrm{L}^{5}}{\alpha v \mathrm{k}}$

$\operatorname{Pr}=\frac{v}{\alpha}$

where
$\mathrm{k} \quad=\quad$ thermal conductivity $(\mathrm{W} / \mathrm{mK})$
$\mathrm{h}=$ average heat transfer coefficient $\left(\mathrm{W} / \mathrm{m}^{2} \mathrm{~K}\right)$
$\mathrm{L}=\quad$ characteristic length $(\mathrm{m})$
g $=$ acceleration due to gravity $\left(\mathrm{m} / \mathrm{s}^{2}\right)$
$\beta=$ volumetric coefficient of thermal expansion $\left(\mathrm{K} / \mathrm{m}^{3}\right)$
$\alpha=$ thermal diffusivity of the fluid in the pool $\left(\mathrm{m}^{2} / \mathrm{s}\right)$
$v \quad=\quad$ kinematic viscosity of the fluid in the pool $\left(\mathrm{m}^{2} / \mathrm{s}\right)$
$q^{\prime \prime \prime}=\quad$ volumetric heat generation rate within the pool $\left(\mathrm{W} / \mathrm{m}^{3}\right)$.

For transient heat transfer, data are typically correlated using the Fourier number, Fo, and the transient Rayleigh number, $\mathrm{Ra}^{\prime}$, which are defined by

$$
\begin{aligned}
& \mathrm{Fo}_{\mathrm{O}}=\frac{\alpha \mathrm{t}}{\mathrm{L}^{2}} \\
& \mathrm{Ra}^{\prime}=\frac{\mathrm{Ra}}{\mathrm{Nu}} .
\end{aligned}
$$

\subsubsection{Typical Ranges for Molten Pool Heat Transfer Parameters}

As part of this study, the range over which the data were obtained was compared to the range of conditions expected during a severe LWR accident. Representative ranges for the dimensionless groups defined in Section 3.2.1 were quantified using representative upper and lower input values and using results from a representative SCDAP/RELAP5/MOD3.1 calculation. Values estimated from these sources are summarized in Table 3-1. 
Upper and lower ranges listed in Table 3-1 were quantified using information in Reference 3-2 and Reference 3-3. These values were obtained by considering a range of debris conditions and reactor designs. Metallic and debris compositions were considered in estimating values for debris thermal properties. Volumetric heat generation rates were quantified using results from previous calculations. Pool characteristic lengths were quantified considering pool sizes possible in the core and in the vessel lower plenum (i.e., upper bounds for the pool radius were based on core and vessel radii).

Table 3-1. Ranges for variables used to estimate pool heat transfer parameters.

\begin{tabular}{|c|c|c|c|}
\hline Parameter/Units & \multicolumn{3}{|c|}{ Condition } \\
\hline Thermal Properties & $\begin{array}{c}\text { Ceramic } \\
\text { Material } \\
\left(\mathbf{U O}_{\mathbf{2}} \text { at } \sim \mathbf{3 2 0 0} \mathbf{K}\right)\end{array}$ & $\begin{array}{c}\text { Metallic Material } \\
\text { (SS-304 at } \sim 1700 \mathrm{~K})\end{array}$ & $\begin{array}{c}80 \% \mathrm{UO}_{2} / 20 \% \\
\mathrm{ZrO}_{2} \text { (by weight) } \\
\text { Compound } \\
\text { (at } \sim 2900 \mathrm{~K})\end{array}$ \\
\hline$\rho, \mathrm{kg} / \mathrm{m}^{3}$ & 8400 & 7250 & 9300 \\
\hline$c_{p}, J / k g K$ & 500 & 560 & 650 \\
\hline $\mathrm{k}, \mathrm{W} / \mathrm{mK}$ & 3.8 & 38 & 3.8 \\
\hline$\beta, \mathrm{K}^{-1}$ & $1.6 \times 10^{-4}$ & $3.5 \times 10^{-4}$ & $7.1 \times 10^{-5}$ \\
\hline$\alpha, \mathrm{m}^{2} / \mathrm{s}$ & $9.0 \times 10^{-7}$ & $9.4 \times 10^{-6}$ & $6.2 \times 10^{-7}$ \\
\hline$v, \mathrm{~m}^{2} / \mathrm{s}$ & $5.4 \times 10^{-7}$ & $3.2 \times 10^{-7}$ & $4.8 \times 10^{-7}$ \\
\hline $\operatorname{Pr}$ & 0.6 & 0.03 & 0.8 \\
\hline $\begin{array}{l}\text { Heat Source and } \\
\text { Geometry }\end{array}$ & \multicolumn{2}{|c|}{ Lower Bound/Upper Bound } & Reference 3-4 \\
\hline$q^{\prime \prime \prime}, W / m^{3}$ & \multirow{2}{*}{\multicolumn{2}{|c|}{$\begin{array}{c}1.0 \times 10^{5} / 2.0 \times 10^{6} \\
1.0 / 2.4\end{array}$}} & $1.98 \times 10^{6}$ \\
\hline $\mathrm{L}, \mathrm{m}$ (core) & & & $<1.5$ \\
\hline $\mathrm{L}, \mathrm{m}$ (vessel) & \multicolumn{2}{|c|}{$1.0 / 2.8$} & Not available \\
\hline
\end{tabular}

In addition, values for these dimensionless groups were quantified based on conditions predicted in a recently completed set of SCDAP/RELAP5/MOD3.1 calculations performed for a Surry station blackout event. ${ }^{3-4}$ In these calculations, a molten pool is predicted to form in the core region at approximately 4.9 hours after reactor scram. At approximately 5.4 hours, the molten material within the pool in the core region (approximately $49,000 \mathrm{~kg}$ of $\mathrm{UO}_{2}$ and $12,000 \mathrm{~kg}$ of $\mathrm{ZrO}_{2}$ ) is predicted to relocate to the lower head, where it forms a second molten pool.

Using values in Table 3-1, the Rayleigh number applicable to pools in the core region is estimated to range from $10^{12}$ to $10^{17}$. For pools that form in the lower head of the vessel, the Rayleigh number is estimated to range from $10^{13}$ to $10^{17}$. Debris Prandtl numbers are estimated to range from 0.03 to 0.8 . As will be discussed in Section 3.2.3, this range of parameters is considerably different that the range for which most natural circulation heat transfer data for fluids with internal heating were obtained. 


\subsubsection{Data for Modeling Molten Pool Natural Convection}

Appendix A reviews a representative sample of experimental and numerical investigations performed to investigate heat transfer from molten pools with volumetric heat sources. In addition to original references reporting natural convection investigations, information in previous reviews (e.g., Reference 3-5 through Reference 3-9) were considered. Although integral data may become available from the planned RASPLAV experiments, ${ }^{3-10}$ information in Appendix A indicates that there are no integral test data available for validating natural convection heat transfer models for severe accident conditions. However, there are considerably more data available for predicting heat transfer from a molten pool than were available at the time that the current model was implemented into SCDAP/RELAP5/MOD3.1.

Data were primarily obtained for single component pools experiencing steady-state natural convection. Both flat and curved geometries were studied for two- and three-dimensional test cavities. In addition, various "fixed" boundary conditions, such as adiabatic and constant temperature conditions, were investigated. The studies are limited in the fluid Prandtl number and Rayleigh number ranges considered. For example, experimental investigations typically use conducting aqueous solutions with a Prandtl number of approximately 7 , which is considerably higher than the 0.03 to 0.8 range estimated for fluids in severe accident molten pools; and Rayleigh numbers in the experimental investigations reviewed are less than $10^{15}$, which is considerably less than the $10^{17}$ upper limit estimated for severe accident molten pools.

This review indicates that there are several phenomena significantly impacting heat transfer from a molten pool for which there are limited data, such as the impact of vapor transport in the pool, multiple components in the pool, melting and solidification at pool boundaries, and the transient natural convection behavior prior to the time period when steady-state natural convection is established. Nevertheless, the available data indicate that significant reductions in modeling uncertainties can be obtained by modifying the existing SCDAP/RELAP5 models for molten pool heat transfer.

\subsubsection{Current SCDAP/RELAP5/MOD3.1 Molten Pool Heat Transfer Model}

For reference purposes, a brief description of the method used to estimate heat transfer from a molten . pool in SCDAP/RELAP5/MOD3.1 is summarized below. As discussed above, SCDAP/RELAP5 can model the formation of a molten pool in two locations: the core region and the vessel lower head. In the core region, various axial and radial locations are divided up into nodes and flow channels; whereas in the vessel lower head, a two-dimensional finite element conduction model, based on the COUPLE code, is used to model the molten pool and vessel. As discussed below, the method used in SCDAP/RELAP5 to estimate heat transfer to the pool boundaries is similar for pools forming in either the core region or the vessel lower head. Potential uncertainties associated with the current method used to predict heat transfer are also identified.

In order to estimate heat transfer from a molten pool, SCDAP/RELAP5 maps the volume of molten material onto a pool with hemispherical geometry. Natural convection heat transfer coefficients from a hemispherical pool are estimated using the following steady-state correlations recommended by Mayinger, Jahn, Reineke, and Steinbrenner. $3-11,3-12$

$N u_{u}=0.36 R a^{0.23}$ 
$\mathrm{Nu}_{\mathrm{d}}=0.54 \mathrm{Ra}^{0.18}$

As discussed in Appendix A, these correlations are based on experimental data obtained from a "slice" hemispherical geometry. In the experiments used to obtain these correlations, the Rayleigh number for the experimental fluid was varied from $1 \times 10^{7}$ to $5 \times 10^{10}$, and the Prandtl number for the experimental fluid, water, was around 7 . Hence, the ranges for which these correlations were obtained are considerably different than the ranges expected for severe accident conditions.

According to data presented by Jahn and Reineke, $3-12$ the heat transfer along the bottom surface varies with location. A maximum downward heat flux, approximately twice the average downward heat flux, occurs at the upper edge of the pool; and the minimum heat flux occurs at the bottom center of the pool. In SCDAP/RELAP5/MOD3.1, the total volume of molten material, irrespective of material composition, is mapped onto a hemispherical shape and heat transfer coefficients estimated for this idealized, hemispherical, geometry are mapped back into the actual configuration of the molten pool. For example, the heat transfer coefficient at the bottom center of the idealized pool is applied to the bottom center of the actual pool.

Note that two of the above simplifying assumptions may lead to erroneous code predictions. First, the assumption that the entire volume of molten material, irrespective of melt composition, can be used to estimate an effective radius for the pool neglects the effects of density-stratified, multiple layers on natural convection. As will be discussed in Section 3.3.4.3, data indicate that models for predicting natural convection in multiple component pools should consider effects such as (a) internal heating present in one or both layers within the pool, (b) the potential for crusts to form at the interface between the layers, and (c) material interactions occurring between the melt layers. Second, the assumption that the molten volume of material within the pool can be mapped onto a hemispherical geometry may lead to erroneous predictions for heat transfer coefficients. Scoping calculations performed in Section 3.4 illustrate the errors that may be introduced when pool geometry effects are ignored.

\subsection{Proposed Modifications for Molten Pool Natural Convection Heat Transfer Model}

Data and analyses pertinent to natural convection in a molten pool are reviewed in Appendix A. In this section, recommendations from this review are summarized. The impact of implementing these recommendations into SCDAP/RELAP5 is assessed in Section 3.4.

\subsubsection{Steady-State Natural Convection}

As discussed in Section 3.2.3, a considerable number of numerical and experimental investigations have been performed to predict steady-state heat transfer from single component molten pools. Figure 3-3 through Figure 3-5 compare correlations obtained from investigations concentrating on fluids with higher Rayleigh numbers. In these figures, correlations are plotted for the range of Rayleigh number for which they were obtained. Labels indicate if correlations were experimentally or analytically obtained. Figure 3-3 compares correlations for predicting upward heat transfer; Figure 3-4 compares correlations for predicting horizontal heat transfer; and Figure 3-5 compares correlations for predicting downward heat transfer. In these figures, curves marked "Mayinger - experimental slice" correspond to correlations 
currently used in SCDAP/RELAP5. A dashed curve, labeled "Recommended," is included in each figure that corresponds to correlations that this review indicates should be used for modeling molten pool behavior in SCDAP/RELAP5. These "Recommended" correlations correspond to data obtained from the Kymäläinen, et al. COPO tests. ${ }^{3-13}$ These tests were obtained for a higher Rayleigh number fluid than other tests (COPO tests investigated Rayleigh number up to $10^{15}$ ). Furthermore, as illustrated in Figures 3-3 through 3-5, these correlations are consistent with many of the correlations recommended from several other two and three dimensional investigations.

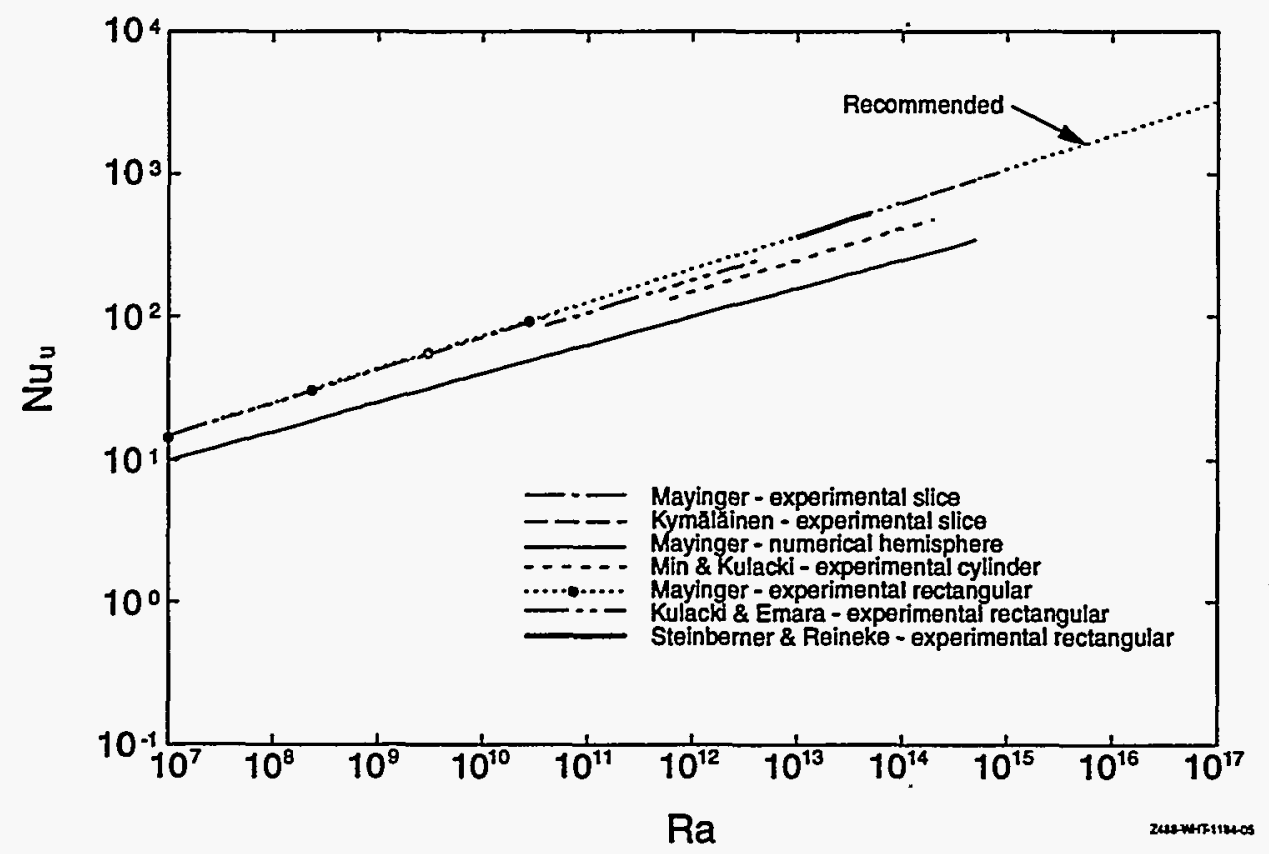

Figure 3-3. Comparison of high Rayleigh number correlations for upward heat transfer (to side walls). 


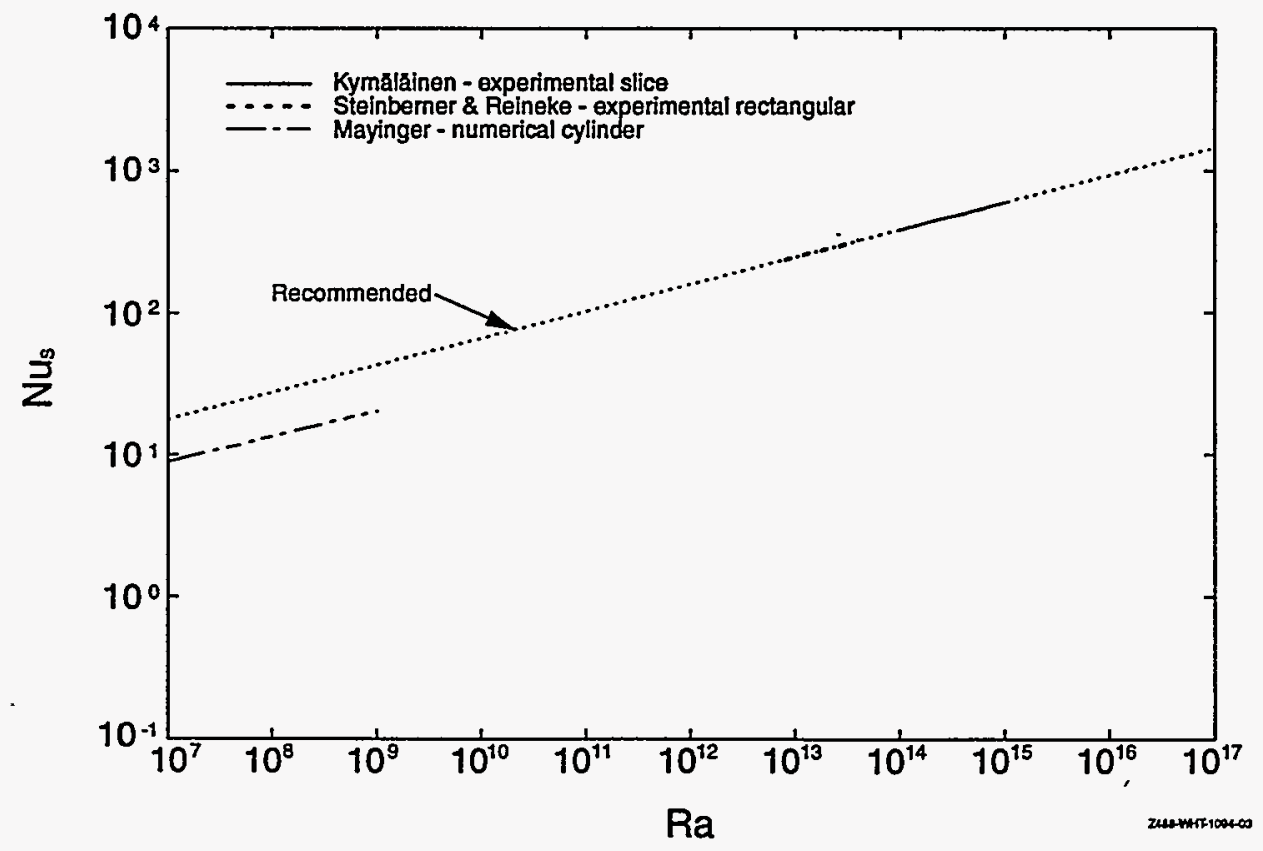

Figure 3-4. Comparison of high Rayleigh number correlations for horizontal heat transfer (to side walls).

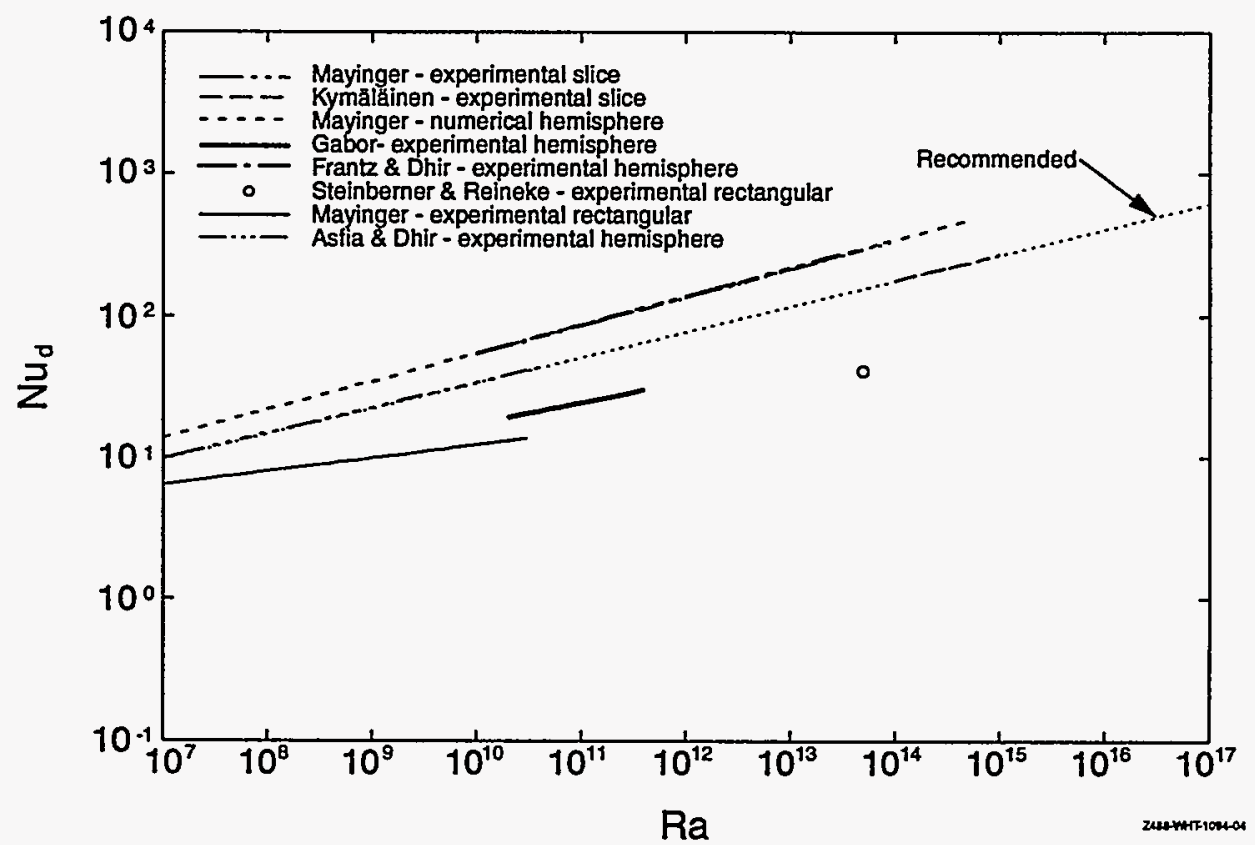

Figure 3-5. Comparison of high Rayleigh number correlations for predicting downward heat transfer. 
It is interesting to note that although the correlations plotted in these figures were obtained from various cavity geometries (rectangular, hemispherical, and slice), there is generally less scatter than from correlations obtained from a single geometry. An obvious exception where more scatter is observed is with correlations obtained for hemispherical geometries (Mayinger ${ }^{3-11}$ - numerical hemisphere, Frantz and $\mathrm{Dhir}^{3-14}$ and Asfia and Dhir ${ }^{3-15}$ - experimental hemisphere, Gabor ${ }^{3-16}$ - experimental hemisphere). Although one might conclude that these differences suggest that two-dimensional data are not applicable to three-dimensional geometries, one should note that there is considerable disagreement among the three-dimensional investigations. Furthermore, a review of References 3-11, 3-14, and 3-16 indicates that there may have been more uncertainty associated with the experimental hemispherical investigations and that the numerical study may have incorrectly modeled turbulent heat transfer. Therefore, the best correlations for predicting steady-state natural convection appear to be the correlations corresponding to the two-dimensional COPO test results, which in many cases are consistent with the Steinberner and Reineke ${ }^{3-17}$ correlations obtained using a three-dimensional rectangular cavity. ${ }^{a}$

For upward and lateral heat transfer, the COPO data were found to be in good agreement with the following correlations proposed by Steinberner and Reineke for heat transfer from a rectangular cavity ${ }^{3-17}$

$\mathrm{Nu}_{\mathrm{u}}=0.345 \mathrm{Ra}^{0.233}$

$\mathrm{Nu}_{\mathrm{s}}=0.85 \mathrm{Ra}^{0.19}$

For the downwards heat flux, the data were found to compare well with the original version of the correlation developed by Mayinger ${ }^{3-11}$

$\mathrm{Nu}_{\mathrm{d}}=0.54 \mathrm{Ra}^{0.18}\left(\frac{\mathrm{H}}{\mathrm{R}}\right)^{0.26}$

where the characteristic length used in the Nusselt and Rayleigh numbers is the height of the curved portion of the test vessel, $H_{c}$. As shown above in Equation (3-30), the original form of the Mayinger correlation includes a geometry correction factor to account for non-hemispherical pools.

Figure 3-6 compares local heat flux measurements for the lower, curved boundary in the Mayinger, et al. slice tests, ${ }^{3-11}$ the Frantz and Dhir hemispherical tests, ${ }^{3-14}$ the Asfia and Dhir hemispherical tests, ${ }^{3-15}$ and the Kymäläinen, et al. COPO slice tests. ${ }^{3-13}$ Although results in Figure 3-6 indicate a significant difference in the manner that the local heat transfer coefficient will vary as a function of angle along the pool's lower surface, the curves are similar in that they go from nearly zero on the bottom surface (at zero degrees) to approximately a factor of 2 or 2.5 at the point where the curved bottom surface intersects the vertical wall (at approximately 90 degrees for the Frantz and Dhir, the Asfia and Dhir $H / R_{e}=1$ tests, and the Mayinger tests, approximately 50 degrees for the Asfia and Dhir $H / R_{e}=0.4$ test, and approximately 45

a. Discussions indicate that the recommended correlations are consistent with preliminary results from recently completed natural convection heat transfer tests at the University of California - Santa Barbara. ${ }^{3-18}$ 
degrees for the Kymäläinen tests). Results from geometries with pool depth to radius ratios less than unity consistently indicate a much steeper rise in local heat transfer. However, results from the torispherical COPO slice tests indicate a much more linear increase in the local heat transfer coefficient than observed in any of the other experiments. Although data from the recently obtained Asfia/Dhir and Frantz/Dhir hemispherical tests are consistent, it should be noted that they were obtained from the same experimental setup. Results from the Asfia and Dhir tests also indicate that upper pool surface boundary conditions also have a slight effect on variations in the heat transfer coefficient.

Because the data for predicting local heat transfer are limited and because the Mayinger curve, which is currently used in SCDAP/RELAP5, appears to give an average of the results obtained from other experimental setups, it is suggested that SCDAP/RELAP5 continue to use the Mayinger results for modeling variations in the local heat transfer coefficient along the bottom surface. Note that the Mayinger results are applied in a manner that allows heat transfer coefficients to rise more rapidly along pool surfaces with pool depth to radius ratios less than unity so that the maximum value of approximately 2 occurs at the point where the curved bottom surface intersects the vertical wall.

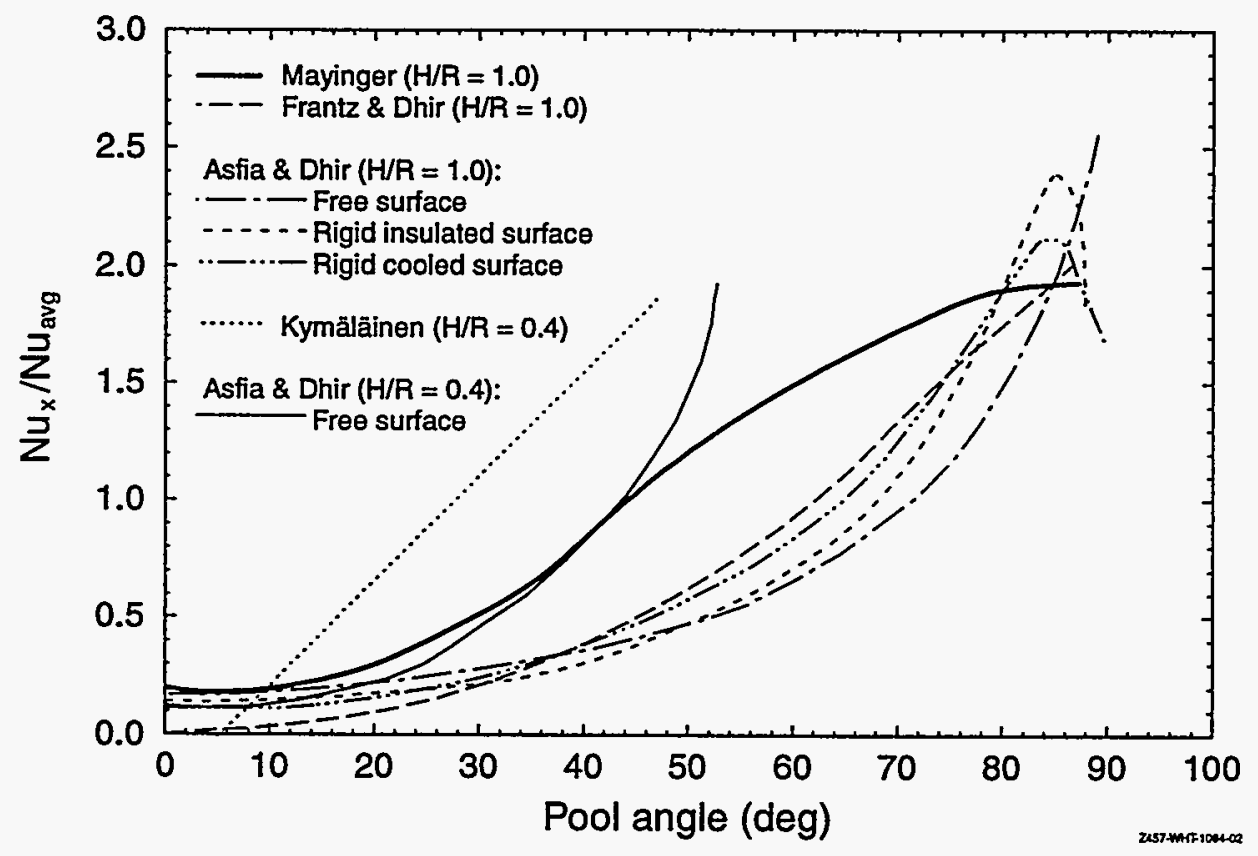

Figure 3-6. Comparison of local heat transfer results.

It should be noted that the proposed recommendations would result in the code's model considering semicircular segment and torispherical geometries rather than mapping the pool's shape onto a hemispherical geometry. As discussed above, this recommendation will be implemented by including a separate correlation for horizontal heat transfer and applying the geometry correction factor suggested by Kymäläinen (and originally in the Mayinger, et al correlation). 
In summary, it is suggested that the steady-state heat transfer correlations used in SCDAP/RELAP5 be modified to reflect data obtained from more recently completed, higher Rayleigh number results from the COPO facility. Note that implementation of this recommendation would allow more general shapes of molten pools to be modeled (from a semicircular segment to a cylinder bounded below by a curved lower surface). Data for predicting variations in local heat transfer on the curved lower surface were also reviewed. However, at this time it is felt that SCDAP/RELAP5 should continue to use the Mayinger data for predicting local values on the lower surface.

\subsubsection{Transient Natural Convection}

For the high Rayleigh numbers that will occur in large pools during a severe accident, experimental evidence indicates that the thermal resistance in convecting regions is determined by relatively thin boundary layers. Thus, several references [References 3-7, 3-19, and 3-20] suggest that it is more appropriate to model heat transfer from such pools using correlations based on the temperatures differences that exist across the convecting region because this approach allows lateral cooling to be included and transient calculations to be performed. Unfortunately, only two experimental investigations reporting transient natural convection data from pools with volumetric heating sources were found in the literature. ${ }^{3-21,3-22}$ In these experiments, correlations relating the pool Fourier number and the step increase or decrease in pool Rayleigh number were obtained. Temperature differences occurring in the pool during the transient time periods were also reported.

Data from these experiments and information in Table.3-1 were used to estimate the time periods required for steady-state natural convection to become established in molten pools that might form during a severe accident. Results indicate that between 1 and 11 hours are required before steady-state natural convection is established. Analyses indicate that transient convection time periods are dependent on pool material composition. For example, the time periods estimated for mixed oxide melt are considerably higher because of the lower thermal diffusivity for this material (estimates range from 6 to 11 hours).

References 3-7, 3-19, and 3-20 describe the following method for modeling heat transfer from a molten pool during the transient and steady-state stages by converting empirical correlations based on a steady-state Rayleigh number to correlations based on a transient Rayleigh number. In general, steady-state correlations have the form

$\mathrm{Nu}=\mathrm{A}_{1} \mathrm{Ra}^{\gamma}$

where the steady-state Nusselt number and the steady-state Rayleigh number are given by

$$
\begin{aligned}
& N u=\frac{q^{\prime \prime \prime} L^{2}}{k \Delta T} \\
& R a=\frac{g \beta q^{\prime \prime \prime} L^{5}}{\alpha v k}=R a^{\prime} N u
\end{aligned}
$$


$\mathrm{Ra}^{\prime}=\frac{\mathrm{Ra}}{\mathrm{Nu}}=\frac{\mathrm{g} \beta \mathrm{L}^{3} \Delta \mathrm{T}}{\alpha \mathrm{V}}$

the correlation given by Equation (3-31) is equivalent to

$\mathrm{Nu}=\left(\mathrm{A}_{1} \mathrm{Ra}^{\prime \gamma}\right)^{\frac{1}{1-\gamma}}$

The above method has been successfully implemented in severe accident analysis codes, such as the MELTPV $^{3-19}$ and PAMPUR ${ }^{3-23}$ codes.

In Appendix A, this method is validated using data obtained by Min and Kulacki for a cylinder bounded below by a spherical segment. In addition, the Min and Kulacki data are used to estimate the error introduced when a steady state correlation is applied to a transient pool. Results indicate that the transient correlation predicts the steady-state experimental data nearly as well as the steady-state correlation (the transient correlation is within $15 \%$ of the experimental values, whereas the steady-state correlation is within $12 \%$ of the experimental values). However, when the steady-state and transient correlations are applied to transient pool data, large differences are observed. Figure 3-7 compares predictions from the transient and steady-state correlation as a function of transient Rayleigh number. In this figure, three transient tests are considered (Runs 149,150, and 151) corresponding to three step increases in the steady-state predictions. While the transient correlation indicates a smooth increase in Nusselt number, corresponding to the temperature increases observed in the pool, the steady-state correlation predicts a constant Nusselt number corresponding to the volumetric heat generation rate in the pool. As illustrated in Figure 3-7, it is not correct to apply a steady-state correlation to predict heat transfer from a transient molten pool. 


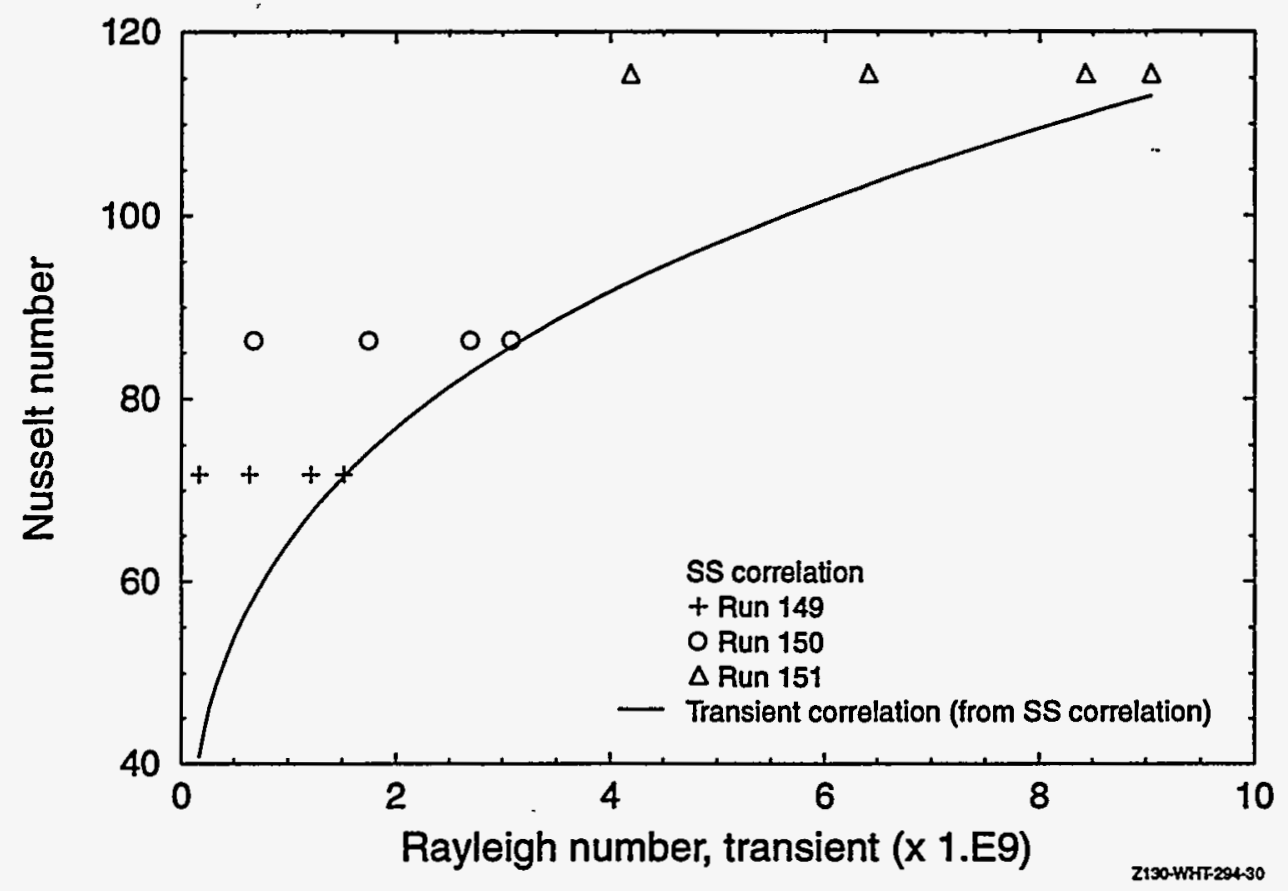

Figure 3-7. Comparison of transient and steady-state correlations as a function of transient Rayleigh number.

In summary, it is recommended that transient correlations be obtained from the steady-state correlations for predicting molten pool heat transfer. Therefore, the steady-state correlations recommended in Section 3.3.1 become

$\mathrm{Nu}_{\mathrm{u}}=0.250 \mathrm{Ra}^{10.304}$

$\mathrm{Nu}_{\mathrm{s}}=0.818 \mathrm{Ra}^{\mathbf{1}^{0.235}}$

$N u_{d}=0.472\left(\frac{H}{\mathrm{R}}\right)^{0.317} \mathrm{Ra}^{0.0220}$.

\subsubsection{Vapor Transport}

If the temperature in the molten pool exceeds the boiling temperatures of one or more of the metallic or oxidic components, vaporization and pool boiling occurs. In addition, the presence of fission products in molten pools formed during severe accidents may lead to gases bubbling up through the melt. There is a limited amount of experimental and theoretical work available in the literature to investigate the effect of boiling on heat transfer from a fluid with internal heating. However, results from these analyses illustrate that the presence of vapors in a molten pool may significantly enhance heat losses from the pool. 
Most studies investigating convective heat transfer with vapor transport concentrate on the heat transfer to side boundaries of the pool. Earlier attempts to model the heat transfer associated with boiling pools attempted to describe this heat transfer as combined natural convection (due to the density differences in the fluid) and forced convection (due to the vapor lift associated with boiling). $3-24,3-25,3-26$ Subsequent works propose that the heat transfer was better described as enhanced natural convection using the following correlation for lateral heat transfer..$^{3-27,3-28}$

$\mathrm{Nu}_{\mathrm{s}}=1.54 \mathrm{Ra}_{\mathrm{b}}^{0.25}$

for $R a_{b}<1.9 \times 10^{11}$, and

$N u_{s}=0.0314 R a_{b}^{0.40}$

for $R a_{b}>1.9 \times 10^{11}$, where the boiling Rayleigh number is defined by

$R a_{b}=\frac{g \cos \theta \alpha^{\prime} L^{3}}{\alpha V}$

where $\alpha^{\prime}$ is the void fraction and $\theta$ is the angle at which the boundary is inclined from the vertical. These correlations were found to agree not only with the more recently obtained data, but also with data obtained from earlier studies. Because these latter correlations were in better agreement with the data and because they are simpler to implement into a systems analysis code, such as SCDAP/RELAP5, it is recommended that heat transfer to sidewalls in pools with boiling be modeled using these latter formulations.

Implementation of this model into SCDAP/RELAP5 requires that the pool void fraction be estimated. This void fraction corresponds to gases associated with fission gas generation and gases associated with pool components that vaporize at lower temperatures. Preliminary review indicates that fission gas generation will primarily occur during early stages of pool formation. Hence, it is recommended that the pool void fraction be based on the quantity of materials vaporize as debris is subsumed into the growing pool. This method is similar to the method currently used in the CORCON code..$^{3-29}$

Studies in this area are limited because they only concentrate on heat transfer to sidewalls. Although additional data are needed for validation, it is recommended that heat transfer to upper and lower surfaces be modeled using the Ginsberg and Greene correlation ${ }^{3-30}$ based on horizontal heat transfer at liquid/liquid interfaces subjected to a gas flux.

$h_{u}=5.05 \frac{k}{r_{b}}\left(\frac{V_{s} r_{b}}{v_{1}}\right)^{0.5} \operatorname{Pr}^{0.8}$ 
where
$h_{u} \quad$ heat transfer coefficient to the upper surface, $W / m^{2} K$
k pool fluid thermal conductivity, W/mK
$r_{b} \quad$ bubble effective radius, $m$
$v_{1} \quad$ pool fluid kinematic viscosity, $\mathrm{m}^{2} / \mathrm{s}$
Pr pool fluid Prandtl number
$\mathrm{V}_{\mathrm{s}} \quad$ bubble superficial vapor velocity, $\mathrm{m} / \mathrm{s}$.

This recommended approach is similar to the method currently used in the CORCON-MOD2 $2^{3-29}$ computer program to predict heat losses from a molten pool containing gases due to concrete decomposition.

Using these recommended correlations, the ratio of the sideward to upward heat transfer coefficient, which is equal to the ratio of the sideward to downward heat transfer coefficient, was estimated. Calculations were performed for a molten pool, containing primarily $\mathrm{UO}_{2}$ for a range of pool void fractions, a range of bubble compositions, and minimum and maximum pool vapor bubble radii (0.0012 and $0.0431 \mathrm{~m}){ }^{3-30}$ In estimating the vapor velocity, $V_{s}$, relationships between the vapor velocity and the terminal vapor bubble velocity, $V_{\infty}$, proposed by Greene, et al. ${ }^{3-27,3-28}$ were applied for various flow regimes. For incipient boiling $(0.00 \leq \bar{\alpha} \leq 0.03)$, bubble nucleation began at a threshold value of $\mathrm{V}_{s} / \mathrm{V}_{\infty}=0.2$. For bubbly flow $(0.03 \leq \bar{\alpha} \leq 0.6), 0.8 \leq \mathrm{V}_{s} / \mathrm{V}_{\infty} \leq 1.0$. For churn turbulent $(\bar{\alpha} \approx 0.4$ after a peak above 0.55$), 1.0<\mathrm{V}_{s} / \mathrm{V}_{\infty}<2.0$. The terminal vapor bubble velocity in a stagnant liquid, $\mathrm{V}_{\infty}$, was estimated by applying

$V_{\infty}=1.4\left[\frac{\sigma g\left(\rho_{1}-\rho_{v}\right)}{\rho_{1}^{2}}\right]^{0.25}$

Results, shown in Figure 3-8, indicate that the upward heat transfer coefficient will be at least a factor of two larger than the sideward heat transfer coefficient for the pool void fractions considered. 


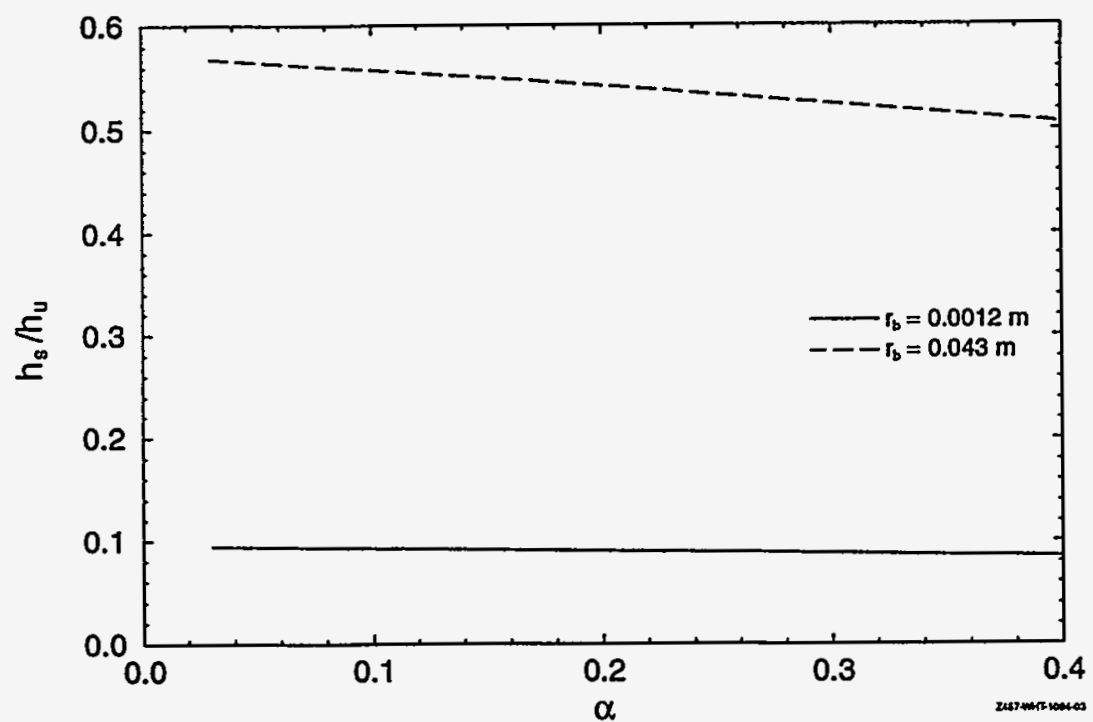

Figure 3-8. Ratios of heat transfer coefficients as a function of pool void fraction.

\subsubsection{Phenomena Requiring Additional Data and/or.Analyses}

This review identified several areas where data indicate that other phenomena may impact molten pool heat transfer, but the data are insufficient to recommend what additional modeling modifications are required to predict these phenomena. These phenomena are summarized in this section.

3.3.4.1 Prototypic Materials. As discussed in Section 3.2.2, the Prandtl number for molten pool material is expected to range between approximately 0.05 and 0.8 . These values are considerably lower than the values for fluids used in experiments reviewed in Section 3.3.1 (typically ranging around 7.0). There are limited data for molten pools containing prototypic materials. . $^{31,3-32,3-33}$ Results from these experiments are considerably different from those using non-prototypic fluids. First, experiments using $\mathrm{UO}_{2}$ have observed that the upward heat flux will be suppressed because of void formation that occurs as the material undergoes a volume reduction as it solidifies. Secondly, an enhanced downward heat transfer was observed in the molten $\mathrm{UO}_{2}$ tests that cannot currently be explained. Although it has been suggested that this enhanced downward heat transfer may be due to an errors in estimating the heatup of the debris or neglecting radiation heat transfer within the debris, additional data are needed to quantitatively estimate what, if any, material property effects may impact convective heat transfer from a molten pool. Some of the OECD-sponsored RASPLAV tests currently planned by the Russian Research Institute (Reference 3-10) will use prototypic materials and may provide insight into the effects of prototypic materials. If such data are obtained from the planned RASPLAV tests, molten pool models in SCDAP/RELAP5 should be reevaluated.

3.3.4.2 Melting and Solidification. There has been limited research on convective heat transfer from a volumetrically heated liquid undergoing a phase change. Note that convective motion . within the liquid may be introduced via several mechanisms: 1) temperature and/or concentration gradients in the presence of a gravitational field (natural convection), 2) the intrinsic density differences associated 
with the transition between the phases and the movement of the solid-liquid front (bulk convection), and 3) the presence of surface tension gradients at the "free" surface of the liquid (surface tension driven convection). Although the effects of natural convection on solidification and melting of metals has been recognized as significant for some time, most of the studies in this area are qualitative rather than quantitative. Results suggest that natural convection significantly impacts the manner in which melting and solidification occurs in a molten pool during a severe accident. The motion in the fluid will enhance heat fluxes (and accelerate melting or decrease solidification) along upper portions of the side walls and the upper walls. Furthermore, data indicate that several phenomena associated with solidification and melting will increase fluid motion within the liquid, such as the increase in fluid volume associated with melting. In this review, limited amounts of quantitative results were found for predicting heat transfer along melting or solidifying surfaces. ${ }^{3-35-3-41}$ These data were obtained for a limited range of conditions, significantly different than those expected for a molten pool during a severe accident. Therefore, it is not recommended that these correlations be incorporated into SCDAP/RELAP5 molten pool models.

In summary, there are several phenomena associated with melting and solidification that are currently included in SCDAP/RELAP5 models. For example, the ability of crusts to form and melt due to heat transfer from the pool is included in SCDAP/RELAP5 models. Furthermore, the change in volume associated with melting and solidification is also considered, although the model assumes that the pool collapses rather than solidifies with voids in it. SCDAP/RELAP5 models do not include any enhanced fluid motion due to melting and solidification phenomena. The models also do not consider the potential for solids to become entrained in the melt and the effects of these entrained solids on heat transfer from the pool. At this time, it is suggested that additional numerical studies and if possible, experimental tests, be performed to investigate the above phenomena before modifications associated with melting and solidification are proposed for SCDAP/RELAP5 molten pool models.

3.3.4.3 Multiple Components or Layers. It has been postulated that multiple component pools may form during a severe accident. For example, there is the potential that control and structural material may relocate earlier than ceramic material or that radiation heat transfer from a large, ceramic pool may cause lower plenum structural material to relocate on top of the ceramic material. When modeling these multiple component pools, it is necessary to determine if there will be natural convection in one or both of the layers. Furthermore, it is necessary to understand what, if any, material interactions are possible that could result in a single homogeneous layer. If it is determined that the layers are immiscible, it is necessary to determine if a crust will form between the layers and estimate the effect of this crust on heat transfer between the layers.

A limited amount of experimental and analytical work has been performed to investigate multiple convecting layers with internal heat sources. ${ }^{3-42-3-45}$ Several investigations indicate that the presence of a nonheated layer above a heated layer reduces heat losses from the convecting layer. No experimental data were found investigating convective heat transfer in multiple layer fluids with only the top layer heated or with both layers heated. ${ }^{a}$ Furthermore, no data were found for modeling material interactions or the impact of crust formation. Nevertheless, it is important to understand how heat transfer will be predicted by SCDAP/RELAP5 when a molten pool forms containing two density-stratified layers. Currently, SCDAP/

a. Discussions indicate that two component, two fluid layer tests will be included in the natural convection heat transfer test program being performed at the University of California - Santa Barbara. ${ }^{3-18}$ These tests will use uniform cooling to simulate internal heat generation in each layer. 
RELAP5 models assume that the volume of the molten pool corresponds to the entire volume of molten material. Using this volume, an effective pool radius is estimated that is then used to calculate the pool Rayleigh number used in steady-state heat transfer correlations. However, the heat transfer correlations are evaluated using the material properties corresponding to the material within each layer. For example, consider a molten pool with an internal heat generation rate of $1.98 \mathrm{MW} / \mathrm{m}^{3}$ consisting of a $2 \mathrm{~m}^{3}$ volume of metallic melt beneath a $4 \mathrm{~m}^{3}$ volume of ceramic melt. The effective radius of the hemispherical pool calculated by SCDAP/RELAP5 would be approximately $0.95 \mathrm{~m}$. Rayleigh numbers estimated for material in the metallic and ceramic region would be $1.3 \times 10^{15}$ and $4.7 \times 10^{13}$, respectively. Heat transfer coefficients along the upper side of the pool would be estimated by SCDAP/RELAP5 using the correlations recommended by Mayinger, Jahn, Reineke, and Steinbrenner. For the upper side of the pool, current SCDAP/RELAP5 models would estimate that the average heat transfer coefficient from the metal

and ceramic materials, would be 12,400 and $2,300 \mathrm{~W} / \mathrm{m}^{2} \mathrm{~K}$, respectively. There are no data with which to refute or substantiate these predictions. Hence, it is strongly recommended that, as a minimum, additional studies be performed to investigate how this phenomena is predicted with more detailed numerical models.

\subsection{Impact of Proposed Modifications}

In Section 3.3, it is proposed that molten pool heat transfer models be modified to improve steady-state modeling and to consider transient time periods and vapor transport. The potential impact of these proposed modifications on SCDAP/RELAP5 predictions has been assessed, and results are summarized below.

\subsubsection{Steady-State Heat Transfer Model Modificaktions}

As discussed in Section 3.2.4, heat transfer from a molten pool is currently modeled in SCDAP/ RELAP5 by applying correlations proposed by Mayinger, et al. In Section 3.3.1, it is recommended that SCDAP/RELAP5 models be updated using the correlations recommended from the Kymäläinen, et al. COPO experiments. These correlations were obtained from higher Rayleigh number experiments. As illustrated in Figure 3-3 through Figure 3-5, these correlations were also found to be consistent with many of the correlations recommended from previous experiments.

As discussed in Section 3.3.1, implementing these correlations would also allow the code to model semicircular segment and torispherical geometries rather than mapping these geometries onto a hemispherical geometry. Scoping calculations were performed to illustrate the effects of accommodating geometry effects using the recommended downward heat transfer correlations. In these calculations, an 80 wt\% $\mathrm{UO}_{2}$ and $20 \mathrm{wt} \% \mathrm{ZrO}_{2}$ mixed oxide compound was assumed. Both semicircular and torispherical geometries were considered. As illustrated below in Figure 3-9, results indicate that heat transfer 
coefficients vary when the correct geometry is considered. These geometry effects were found to impact the heat transfer coefficient by as much as $20 \%$.

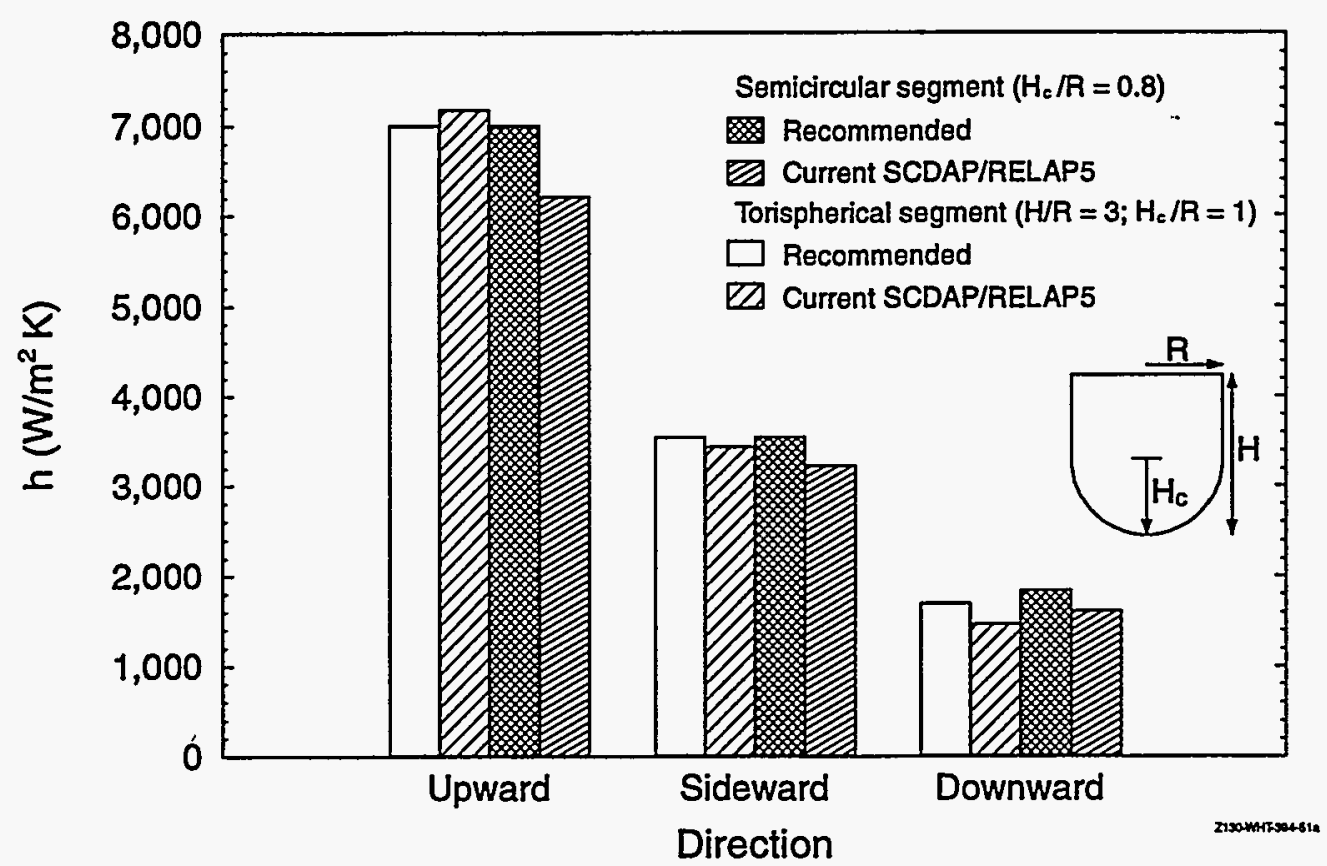

Figure 3-9. Comparison of heat transfer coefficients predicted when geometry effects are considered.

\subsubsection{Transient Model Modifications}

In Section 3.3.2, it is recommended that transient forms of steady-state natural convection heat transfer correlations be implemented into SCDAP/RELAP5. To illustrate the importance of using transient forms of these correlations, calculations were performed comparing transient and steady-state heat transfer correlation predictions assuming the molten pool geometry and composition predicted in the SCDAP/ RELAP5/MOD3.1 analysis described in Section 3.2.2. In these calculations, a hemispherical geometry was assumed and the following steady-state heat transfer correlation currently in SCDAP/RELAP5 for predicting downward heat transfer was applied.

$\mathrm{Nu}_{\mathrm{d}}=0.54 \mathrm{Ra}^{0.18}$

Using the method described in Section 3.3.2, this correlation translates to the following transient correlation

$N u_{d}=0.47 \mathrm{Ra}^{10.22}$

Transient and steady-state Nusselt numbers for the pool that forms in the core region at 17,500 seconds were estimated using parameters, such as the pool temperatures, characteristic lengths, volumetric heat generation rate and melt material composition for the SCDAP/RELAP5 analyses described in 
Section 3.2.2. Results are compared below in Figure 3-10. As illustrated in Figure 3-10, the Nusselt number predicted with the steady-state correlation is significantly higher (at least a factor of two) than that predicted with the transient correlation.

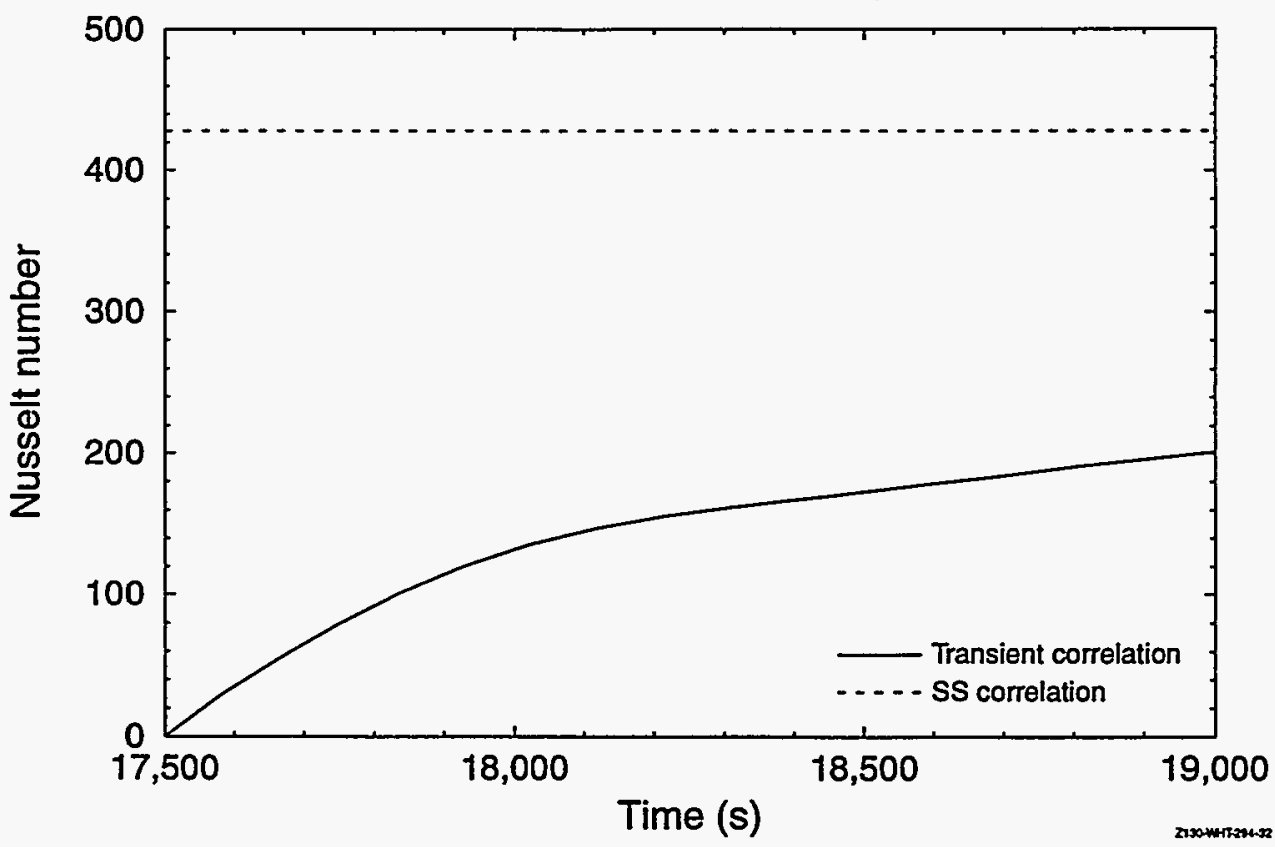

Figure 3-10. Comparison of the transient and steady-state Nusselt numbers for the Section 3.2.2 SCDAP/RELAP5 molten pool.

Information in Section 3.3.2 indicates that a pool containing a mixed oxide melt will not reach a steady-state until after six hours, whereas the SCDAP/RELAP5 calculation discussed in Section 3.2.2 indicates that molten pool material will break through its ceramic crust and relocate to the lower head within 0.6 hours after formation. Thus, it appears that transient time periods may be of more interest to severe accident analyses and that steady-state correlations greatly overpredict heat transfer for transient time periods. Hence, SCDAP/RELAP5 modeling uncertainties will be greatly reduced if molten pool heat transfer correlations are implemented in their transient forms.

\subsubsection{Vapor Transport Model Modifications}

In Section 3.3.3, it is recommended that SCDAP/RELAP5 molten pool heat transfer models be modified to consider vapor transport. To illustrate the possible impact that vapor transport may have on natural convection heat transfer, the correlations developed by Greene, et al. ${ }^{3-27,3-28}$ for predicting horizontal heat losses were applied to the $80 \% \mathrm{UO}_{2} / 20 \% \mathrm{ZrO}_{2}$ weight fraction pool that was predicted to form in the core region in Reference 3-4, SCDAP/RELAP5/MOD3.1 calculations, discussed in Section 3.2.2. The heat transfer predicted by Equations (3-39) and (3-40) are compared with values predicted by Equation (3-29), which was recommended by Kymäläinen, et al. ${ }^{3-13}$ for heat transfer to the side wall. Results, which are shown below in Figure 3-11, indicate that heat transfer may be increased by more than an order of magnitude, depending on the pool's Rayleigh number and the void fraction in the 
pool. For example, if one considers the pool Rayleigh number of $10^{16}$ predicted in the Reference $3-4$ calculations described in Section 3.2.2 and assumes the void fraction for the melt corresponds to the $20 \%$ porosity estimated for samples of debris material found in the TMI-2 lower head, ${ }^{3-46}$ the heat transfer to the side walls is estimated to increase by nearly a factor of 30 .

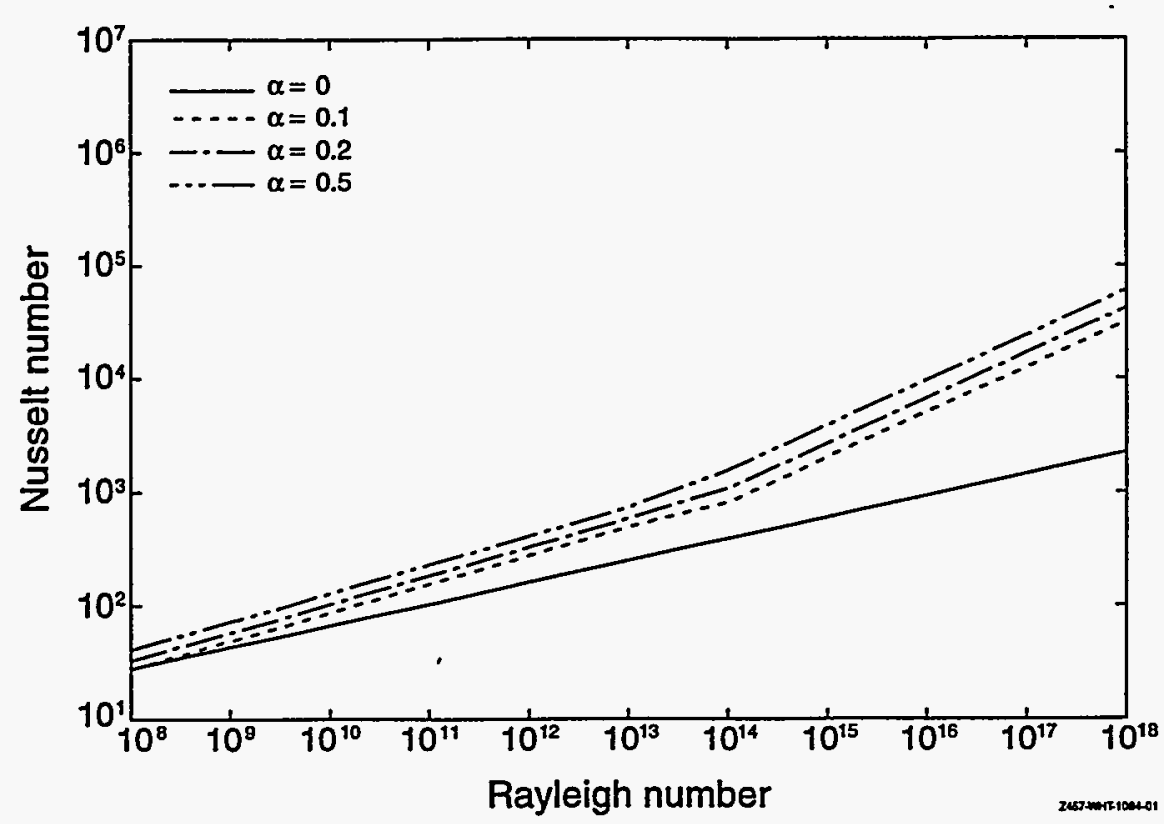

Figure 3-11. Impact of boiling on heat transfer from a molten pool.

\subsection{Summary and Recommendations}

In this section, recommendations for revising SCDAP/RELAP5 molten pool heat transfer models are also summarized and areas where additional data are needed before revisions to SCDAP/RELAP5 heat transfer model can be recommended are identified. Because there are no integral test data available to assess the revised model, steps for numerically assessing the revised model are recommended. These steps are summarized in Section 5.

Based on this review, it is judged that there are sufficient data to warrant recommending the following modifications to the SCDAP/RELAP5 molten pool heat transfer models.

- Use steady-state heat transfer correlations obtained from the Kymäläinen, et al. COPO tests. The correlations recommended by Kymäläinen, et al. for predicting upward and downward heat transfer are similar to those used in the current SCDAP/RELAP5 model. However, it is recommended that these correlations be used because they were obtained from higher Rayleigh number tests and they are consistent with data obtained from several other two- and three-dimensional investigations. Switching to these correlations would include the use of a separate, horizontal, heat transfer coefficient and allow non-hemispherical geometries to be modeled. It is recommended that the Mayinger data for predicting local heat transfer coefficients along curved lower surface of a pool continue to be used in the SCDAP/RELAP5 model. 
- Consider cylindrical and hemispherical pool shapes and aspect ratios. By mapping the volume of a molten pool onto a hemispherical shape, SCDAP/RELAP5 models may be erroneously predicting sideward heat losses. Scoping calculations in Section 3.4.1 indicate heat transfer coefficients may vary by as much as $20 \%$ when the proper geometry of the pool is considered. Thus, it is suggested that shape-factor selection criteria be included in SCDAP/RELAP5 to determine if the melt region is torispherical or hemispheric and the aspect ratio associated with the selected geometry. These geometrical parameters could then be used to appropriately apply the Kymäläinen, et al. COPO correlations.

Apply steady-state correlations in their transient form. As demonstrated in this study, it is incorrect to apply steady-state correlations before the pool has reached a steady-state because heat transfer coefficients will be overpredicted by over a factor of 2 (and possibly orders of magnitude at early stages of the transient). Furthermore, representative results from a SCDAP/RELAP5/MOD3.1 run indicates that molten pools will be in a transient stage for most time periods of interest in a severe accident analysis. Hence, it is recommended that the steady-state Kymäläinen, et al. COPO correlations be applied using their transient forms in SCDAP/RELAP5 calculations.

Consider the effects of vapor transport on convection heat transfer. Although additional data are needed to validate the proposed modifications, it is suggested that horizontal heat transfer be modeled using correlations proposed by Greene, et al. and that upward and downward heat transfer be modeled using the Ginsberg and Greene correlation. Use of this method requires selecting a boiling regime based on the average void fraction of the melt and calculating a bubble terminal vapor velocity. It is recommended that the pool void fraction be based on the quantity materials that vaporize as debris is subsumed into the growing pool. Scoping calculations in Section 3.4.3 indicate that neglecting vapor transport within the melt may result in underpredicting heat transfer from the pool by orders of magnitude.

The following areas have been identified as having the potential to significantly impact heat transfer from a molten pool although additional analyses and/or data are required before any model modifications could be recommended.

Prototypic material property effects. The limited number of experiments performed using prototypic material properties suggest that heat transfer from a molten ceramic pool may behave differently than indicated from experiments using aqueous solutions. First, experiments using $\mathrm{UO}_{2}$ (and experiments using paraffin) have observed that the upward heat flux will be suppressed because of void formation that occurs as the material undergoes a volume reduction as it solidifies. Secondly, an enhanced downward heat transfer was observed in the molten $\mathrm{UO}_{2}$ tests that cannot currently be explained. Although it has been suggested that this enhanced downward heat transfer may be due to an errors in estimating the heatup of the debris or neglecting radiation heat transfer within the debris, additional data are needed to quantitatively estimate what, if any, material property effects may impact convective heat transfer from a molten pool. Note that some of the RASPLAV tests currently planned by the Russian Research Institute will use prototypic materials and may provide insight into this area. 
Solidification and melting effects on fluid motion. Although no data were found that considered the effects of solidification and melting in a volumetrically heated liquid, the data for fluids without heating indicate several effects of phase change that are not considered in SCDAP/RELAP5 molten pool heat transfer models. For example the increase in volume associated with melting has been observed to induce motion in the fluid. Furthermore, SCDAP/RELAP5 models do not consider the potential for solids to become entrained in the melt and the effect of these entrained solids on heat transfer from the pool. Finally, SCDAP/RELAP5 models do not consider the potential for voids to form in the crust as the melt solidifies. It is suggested that insights could be gained in many of these phenomena by performing additional numerical studies.

The effects of multiple-component or layer pools. The limited data available for convection in multiple component fluids suggest that heat transfer at the boundary of the heated layer is independent of the presence of an overlying non-heated layer. Furthermore, the data available for a non-heated layer above a heated layer indicate that upward heat transfer from the two layers is reduced because of the presence of the non-heated layer. No data were available for studying heat transfer from multiple layers with the heating in both layers or the top layer. Data were also not available to study the effects of crust formation or material interactions at the interface between the layers. Although the data for predicting heat transfer from multiple component pools are insufficient to recommend any SCDAP/ RELAP5 model modifications, scoping calculations discussed in Section 3.3.4.3 indicate that the current manner in which SCDAP/RELAP5 molten pool heat transfer models treat multiple component pools is unsubstantiated and it is recommended that calculations be performed to substantiate these predictions using more detailed codes. Furthermore, data from two component, two fluid layer tests that are planned in a natural convection heat transfer tests program being performed at the University of California - Santa Barbara ${ }^{3-18}$ may provide some insight into this area.

\subsection{References}

3-1. C. M. Allison, et al., SCDAP/RELAP5/MOD3.1 Code Manual, Volume II: Damage Progression Model Theory, NUREG/CR-6150, EGG-2720, October 1993

3-2. J. L. Rempe, et al., Light Water Reactor Lower Head Failure Analysis, NUREG/CR-5642, EGG-2618, October 1993.

3-3. C. M. Allison, et al., SCDAP/RELAP5/MOD3.1 Code Manual, Volume IV: MATPRO - A Library of Materials Properties for Light-Water-Reactor Accident Analysis, NUREG/CR-6150, EGG-2720, November 1993.

3-4. C. M. Allison, et. al., SCDAP/RELAP5/MOD3.1 Code Manual, Volume V: Developmental Assessment, NUREG/CR-6150, EGG-2720, December 1993.

3-5. K. M. Kelkar, R. C. Schmidt, and S. V. Patankar, "Numerical Analysis of Laminar Natural Convection for an Internally Heated Fluid in a Hemispherical Cavity," ANS Proceedings of the 1992 National Heat Transfer Conference, Volume 6, pp. 355-364, San Diego, CA, August 1992.

3-6. F. A. Kulacki, Review and Evaluation of 'Examination of Thermohydraulic Processes and Heat Transfer in a Core Melt, ' by Mayinger, Jahn, Reineke, and Steinbrenner, Letter report prepared for the U.S. NRC under contract AT(49-24)-0149, March 31, 1976. 
3-7. "Compendium of Post Accident Heat Removal Models for Liquid Metal Cooled Fast Breeder Reactors," Edited by B. D. Turland and J. Morgan, European Applied Research Reports, Vol. 6, No. 5, April 1985.

3-8. F. A. Kulacki and D. E. Richards, "Natural Convection in Plane Layers and Cavities with Volumetric Energy Sources," Natural Convection, Fundamentals and Applications, Edited by S. Kakaç, W. Aung, and R. Viskanta, Hemisphere Publishing corporation, Washington, D. C., 1985.

3-9. U. Müller and T. Schulenberg, Post-Accident Heat Removal Research: A State of the Art Review, KfK 3601, November, 1983.

3-10. J. Royen, "The RASPLAV Programme," Second CORVIS Task Force Meeting, Paul Scherrer Institute (PSI), Villigen, Switzerland, July 8-9, 1993.

3-11. F. Mayinger, M. Jahn, H. H. Reineke, and V. Steinbrenner, "Examination of Thermalhydraulic Processes and Heat Transfer in a Core Melt," BMFT RS 48/1, Institut für Verfahrenstecknik der T. U., Hanover FRG (in German), 1976.

3-12. M. Jahn and H. H. Reineke, "Free Convection Heat Transfer with Internal Heat Sources," 5th Int. Heat Transfer Conference, Paper NC2.8, Tokyo, Japan, 1974.

3-13. O. Kymäläinen, H. Tuomisto, O. Hongisto, and T. G. Theofanous, "Heat Flux Distribution from a Volumetrically Heated Pool with High Rayleigh Number," NURETH-6 Meeting, Grenoble, France, October 1993.

3-14. B. Frantz and V. Dhir, "Experimental Investigation of Natural Convection in Spherical Segments of Volumetrically Heated Pools" ASME Proceedings of the 1992 National Heat Transfer Conference, HTD-Vol. 192, Thermal Hydraulics of Severe Accidents, San Diego, CA, August 1992.

3-15. F. J. Asfia and V. K. Dhir, "Natural Convection Heat Transfer in Volumetrically Heated Spherical Pools," CSNI Workshop on Large Molten Pool Heat Transfer, Grenoble, France, March 1994.

3-16. J. D. Gabor, J. C. Cassulo, and P. C. Ellison, "Heat Transfer from Internally Heated Hemispherical Pools," 19th National Heat Transfer Conference, Orlando, FL, July 1980, ASME Paper 80-HAT-16.

3-17. U. Steinberner and H. H. Reineke, "Turbulent Buoyancy Convection Heat Transfer with Internal Heat Sources," Proc. 6th Int. Heat Transfer Conf., Vol 2, pp. 305-310, Paper NC-21, Toronto, Canada, August 1978.

3-18. T. Theofanous, University of California - Santa Barbara, Santa Barbara, CA, personal conversation with J. L. Rempe, Idaho National Engineering Laboratory, Idaho Falls, ID, October 5, 1994.

3-19. B. D. Turland and J. Morgan, "Thermal Attack of Core Debris on a PWR Reactor Vessel," Proceedings of the International Meeting on Light Water Reactor Severe Accident Evaluation, August 28-September 1, 1983, Cambridge, MA.

3-20. B. D. Turland and J. Morgan, "Progression of a PWR Severe Accident from Core Melt to Cavity Interactions," EPRI-NP-4455.

3-21. F. A. Kulacki and A. A. Emara, "Steady and Transient Thermal Convection in a Fluid Layer with Uniform Volumetric Energy Sources,” Journal of Fluid Mechanics, 83, 1977, pp. 375-395.

3-22. J.H. Min and F. A. Kulacki, Steady and Transient Natural Convection with Volumetric Energy Sources in a Fluid Layer Bounded from Below by a Segment of a Sphere - Annual Report 6, July 1976- September 1977, NUREG/CR-0006, February, 1978. 
3-23. R. S. Peckover, B. D. Turland, and R. T. P. Whipple, "On the growth of melting pools in Sacrificial Materials," Proceedings of the 3rd Post Accident Heat Removal Information Exchange, Argonne, 1977 (ANL-78-10).

3-24. J. D. Gabor, L. Baker, Jr., J. C. Cassulo, and G. A. Mansoori, "Heat Transfer from Heat-Generating Boiling Pools," AIChE Symposium Series, 164, Vol. 73, p. 73-85, 1977.

3-25. W. R. Gustavson, J. C. Chen, and M. S. Kazimi, Heat Transfer and Fluid Dynamic Characteristics of Internally Heated Boiling Pools, BNL-NUREG-50722, September 1977.

3-26. W. R. Gustavson, M. S. Kazimi, and J. C. Chen, "Heat Transfer and Fluid Dynamics in a Volume-Heated Boiling Pool," Proceedings of the ANS-ENS International Meeting on Fast Reactor Safety and Related Physics, Chicago, IL, USERDA report CONF-761001, p. 2066-2076.

3-27. G. A. Greene, O. C. Jones Jr., C. E. Schwartz, and N. Abuaf, Heat Removal Characteristics of Volume Heated Boiling Pools with Inclined Boundaries, NUREG/CR-1357, BNL-NUREG-51157, 1980.

3-28. G. A. Greene and C. E. Schwartz, Boiling Pools with Internal Heat Generation, NUREG-CR-1249, pp 90-96, January 1980.

3-29. R. K. Cole, Jr., D. P. Kelly, and M. A. Ellis, CORCON-MOD2: A Computer Program for Analysis of Molten-Core Concrete Interactions, NUREG/CR-3920, SAND84-1246, August 1984.

3-30. T. Ginsberg and G. A. Greene, "BNL Program in Support of LWR Degraded Core Accident Analysis," Proceedings of the U. S. Nuclear Regulatory Commission Tenth Water Reactor Safety Research Information Meeting, NUREG/CP-0041, Vol. 2, pp 364-395, 1983.

3-31. L. Baker Jr., M. G. Chasanov, J. D. Gabor, W. H. Gunther, J. C. Cassulo, J. D. Bingle, and G. A. Mansoori, "Heat Removal from Molten Pools," Proc. of the ANS-ENS International Meeting on Fast Reactor Safety and Related Physics, Chicago, IL, USERDA Report CONF-761001 (1976).

3-32. M. G. Chasanov, W. H. Gunther, and L. Baker, Jr., "The Production of Molten $\mathrm{UO}_{2}$ Pools by Internal Heating: Apparatus and Preliminary Experimental Heat Transfer Results," Nuclear Technology, 36, pp. 120-128 (1977).

3-33. R. P. Stein, L. Baker, Jr., W. H. Gunther, and C. Cook, "Heat Transfer from Internally-Heated Molten $\mathrm{UO}_{2}$ Pools," Proc. Fourth Post Accident Heat Removal Information Exchange, pp. 1468-1472 (1979).

3-34. R. Viskanta, "Natural Convection in Melting and Solidification," Natural Convection, Fundamentals and Applications, Edited by S. Kakaç, W. Aung, and R. Viskanta, Hemisphere Publishing Corporation, Washington, D. C., 1985.

3-35. R. D. White, A. G. Bahelt, W. Leidenfrost, and R. Viskanta, "Study of Heat Transfer and Melting from a Cylinder Imbedded in a Phase Change Material," ASME Paper No. 77-HT-42, 1977.

3-36. A. G. Bathelt and R. Viskanta, "Heat Transfer at the Solid-Liquid Interface during Melting from a Horizontal Cylinder," International Journal of Heat and Mass Transfer, 23, pp. 493-1503, 1980.

3-37. A. G. Bathelt, R. Viskanta, and W. Leidenfrost, "Latent Heat-of-Fusion Energy Storage: Experiments on Heat Transfer from Cylinders during Melting," Journal of Heat Transfer, 101, pp. 453-458, August 1979.

3-38. C.-J. Ho and R. Viskanta, "Heat Transfer during Melting from an Isothermal Vertical Wall," Transactions of the ASME, 106, February 1984. 
3-39. E. M. Sparrow and J. A. Broadbent, "Freezing in a Vertical Tube," Journal of Heat Transfer, 105, pp. 217-225, 1983.

3-40. E. M. Sparrow and P. S. Mendes, "Natural Convection Heat Transfer Coefficients Measured in Experiments on Freezing," International Journal of Heat and Mass Transfer, 25, pp. 293-297, 1982.

3-41. R. G. Kemink and E. M. Sparrow, "Heat Transfer Coefficients for Melting about a Vertical Cylinder with or without Subcooling and for Open or Closed Containment," International Journal of Heat and Mass Transfer, 24, pp. 1699-1710, 1981.

3-42. G. Fieg, "Experimental Investigations of Heat Transfer Characteristics in Liquid Layers with Internal Heat Sources," Proceedings of the ANS-ENS International Meeting on Fast Reactor Safety and Related Physics, Chicago, IL, USERDA Report CONF-761001-P4, pp. 2047-2055, 1976.

3-43. R. Schramm and H. H. Reineke, "Natural Convection in a Horizontal Layer of two Different Fluids with Internal Heat Sources," Proceedings of the Sixth International Heat Transfer Conference, Toronto, Canada, Vol. 2, Paper NC-20, pp 299-304, 1976.

3-44. F. A. Kulacki, A. A. Emara, J. H. Min, and A-T. Nguyen, "Experimental Studies of Steady and Transient Natural Convection with Internal Heat Sources in Enclosed Cavities," Proceedings of the Fourth Water Reactor Safety Research Meeting, Gaithersburg, MD, 1976.

3-45. F. A. Kulacki and A-T Nguyen, Hydrodynamic Instability and Thermal Convection in a Horizontal Layer of Two Immiscible Fluids with Internal Heat Generation, NUREG/CR-2619R3, 1980.

3-46. J. R. Wolf and J. L. Rempe, TMI-2 Vessel Investigation Project Integration Report, OECD-NEA-TMI-2 Vessel Investigation Project Report TMI V(93)EG10, October 1993. 


\section{MOLTEN POOL CRUST FAILURE}

This section briefly reviews data from the TMI-2 accident concerning crust failure, current thermally-based crust failure models, a proposed new model that better represents both the thermal and mechanical aspects of crust failure, and the implications of the implementation of the new model. In addition, this section presents a series of parametric studies showing the type of behavior that would be predicted by the new model under TMI-2-like accident conditions.

Although this new model will clearly represent more of the critical processes associated with crust failure, it must be noted that the precise timing and location of the failure will still have relatively large uncertainties, due not only to the limited data base for assessment, but due to the possible random nonhomogeneity of the crust thickness and composition, lack of high temperature mechanical properties for the crust, and possible influence of cracking of the crust if it is exposed to water (as occurred in TMI-2). As a result, a bounding approach is recommended that will allow the user to bound the mass or energy of the melt relocating after crust failure. That is, the factors contributing to the uncertainties in crust failure will be established during the developmental assessment process and grouped in a consistent manner so that a reasonable upper and lower bound on the crust failure timing and location can be established easily by the code user. This bounding approach is also consistent with the bounding approach used in the current slumping models. As a result, the user should be able to quickly establish an upper and lower bound on the melt mass and energy at lower head failure as a result of uncertainties in crust failure and melt slumping processes by selecting a single option in the code.

\subsection{Review of Crust Data from the TMI-2 Accident}

Tolman et al. ${ }^{4-1}$ examined core bore samples from the severely damaged TMI-2 core. These examinations indicate that by 174 minutes into the accident, relocated core materials had formed a large region of nearly solid structure in the core. At this time, the B pump transient injected coolant into the reactor vessel. Resulting thermal mechanical forces are hypothesized to have shattered remaining upper oxidized fuel rod remnants above the molten core region, forming a rubble bed on top of the solid structure. The solid structure of core material near the bottom of the core continued to heat, increasing the amount of molten material in the central core. The molten material was contained within the original core boundary by a crust of solidified ceramic material at the outer edge of this solid structure of core materials.

At approximately 224 minutes a major core relocation event occurred, with molten material relocating from the east side of the core. Epstein and Fauske ${ }^{4-2}$ reason that the actual relocation event followed the collapse of the thin upper crust under the weight of the overlying rubble bed. The rubble bed displaced the ceramic melt and melt material flowed downward to the lower plenum. One dimensional thermal analysis of crust failure by Kuan and Tolman ${ }^{4-3}$ also suggests that upper crust failure was more likely than other locations.

The TMI-2 crust can be sectioned into 3 parts: top, bottom and side. Prior to relocation, the top crust consisted of previously molten ceramics and metallics. Peak temperature of the previously molten ceramic was at least $2800 \mathrm{~K}$. The side crust consisted of previously molten ceramics and metallics, similar to the upper crust, and solid $\mathrm{UO}_{2}$ particles. The ceramics and metallics had similar composition to the upper crust composition, with the same peak temperature for previously molten ceramic of at least $2800 \mathrm{~K}$. The bottom crust consisted of $\mathrm{UO}_{2}$ pellets and small, solid $\mathrm{UO}_{2}$ particles surrounded by previously molten structural 
and control materials. The peak temperature of the lower crust was between 1400 and $2200 \mathrm{~K}^{4-4}$ Note that thermal considerations demand that the upper inside surface of this crust must also have had a layer of frozen ceramic between it and the (interior) molten mass. ${ }^{4-5}$ The thickness of the crust, particularly the side crust, varied widely. $4-6,4-7$

Structural and thermal analyses performed by $\mathrm{Kuan}^{4-8}$ indicate several important points concerning crust behavior. Simple thermal analysis, which conservatively balances decay heat with only radiation heat losses to coolant, predicts an outside crust surface temperature of $1710 \mathrm{~K}$. This precludes melt-through and indicates a high thermal gradient through the crust wall (inside crust wall temperature must be the liquidus temperature). Elastic thermal stress analysis using $1710 \mathrm{~K}$ surface temperature indicates that the bottom crust should reach ultimate strength when its thickness is greater than $3 \mathrm{~cm}$. Because thermal stresses are self-limiting (deformation relieves the stress), they cannot cause failure. Indeed, the bottom crust did not fail in the accident. Apparently the additional pressure stresses (which are not relieved with deformation) were not high enough to cause the bottom crust to fail.

To summarize the structural aspects of crust failure for TMI-2: a non-homogeneous, primarily ceramic crucible encased a region of molten debris. The crucible top supported a sizable debris load; the crucible bottom had a outer lining of primarily metallic material that was unlikely to fail under TMI-2 conditions. The crucible wall varied widely in thickness and had a high temperature gradient through the thickness. It was subjected to thermal and pressure stresses. Melting temperature and strength may have been reduced due to eutectic formation, in particular the reaction between zirconium and nickel (in stainless steel baffle and Inconel grid spacer). Possible failure modes include melting, exceeding ultimate strength, creep, fracture and ductile tearing. However, simple analysis of the TMI-2 accident conditions indicate that, in this case, melt-through and fracture were unlikely.

\subsection{Review of Existing Crust Failure Models}

The current code allows for molten pool spreading while enclosed by a crust and core slumping to the lower head region. Molten pool spreading is based on crust stability, as determined by the relative values of heat fluxes on the inner and outer surfaces of the crust. If the heat flux on the inner surface exceeds the outer surface, the crust is considered unstable and the molten pool spreads radially and downward. If inner surface heat flux does not exceed the outer surface heat flux, the crust is considered stable and the spreading rate equals zero. Downward spreading also halts if the molten pool encounters a metallic crust that formed during the metallic meltdown of the core. The metallic crust is considered to fail when it has melted to a thickness of $25 \mathrm{~mm}$, at which time the molten pool continues to spread downward. There is also a user option to specify a different metallic crust thickness at failure and to have the molten pool slump to the lower head at the instant of metallic crust failure. ${ }^{4-9}$

The current code considers three mechanisms to trigger slumping from the core region to the lower head of the reactor vessel: melting of the side crust once it reaches the periphery of the core, failure of the top crust once its thickness is less than $5 \mathrm{~mm}$ and the molten pool has spread to the periphery of the core, and propagation of the molten pool to the elevation of the bottom of the core. There is also a provision for a user-specified slump at the instant the molten pool reaches the periphery of the core. In all events that trigger slumping of the molten pool, the code considers the entire molten pool to slump to the lower head. ${ }^{4-}$ 9 
In summary, molten pool spreading and core slumping are based on heat flux, melt, or crust thickness. Intuitively important stuctural considerations, such as applied load and temperature-dependent strength, are not included.

\subsection{Proposed Model Improvements}

In the proposed models, the molten pool is assumed to be contained in a crucible of cylindrical geometry made of resolidified debris. Failures are assumed to occur in the top "lid" of the crucible or in the crucible side-wall. The models calculate stresses in a dome top crust and cylindrical side-wall crust, predicting failure when the effective stresses exceed temperature-dependent ultimate strengths.

Side-wall failure is always expected before bottom crust failure (see Appendix B). Therefore, failure is not considered for the bottom until its temperature is greater than or equal to the temperature corresponding to zero ultimate strength (approximately $2251 \mathrm{~K}$ ). Analyses of crust side-wall and bottom stresses indicates the side-wall stress is approximately 20 times higher than the bottom crust, using a thickness of $1 \mathrm{~cm}$ for both and assuming that only one out of four fuel pins remains intact to support the bottom crust. For $20 \mathrm{~cm}$ thick walls with only one out of four fuel pins supporting the bottom crust, the side-wall stress is approximately 90 times higher. In addition to this huge margin, the crucible bottom is likely to be cooler and thicker than side-walls.

Thermal stresses for steady state or quench conditions are not considered in this analysis. Current models assume the crust side walls expand through melt and resolidification. This implies a continuous annealing of thermal stresses. Thermal stresses are self-limiting (deformation relieves stress). Steady state thermal stress predictions based on a static geometry would not be consistent with crust growth. Thermal stress induced by quench could conceivably initiate fracture. Creep failure is another failure mode not considered. Unfortunately, neither creep nor fracture properties of resolidified debris are well-quantified.

Acknowledging variability in crust composition, lack of experimental information on crust failure mode, and lack of material properties, a detailed model of crust failure would be inappropriate. Until experimental failure mode and material property data on resolidified debris become available, a simplified stress analysis, which uses ultimate strength of $\mathrm{UO}_{2}$ as a failure criteria, is proposed.

\subsubsection{Bounding Cases for Analyzing Stresses}

A bounding approach is used to quantify stresses because of large uncertainties in crust permeability and thickness, and temperature-dependent ultimate strength. The proposed core slumping models evaluate pressure stresses using a bounding approach to deliver minimum and maximum energy per slump to the lower head. Assuming an eventual crust failure is unavoidable, a single, delayed side-wall failure would deliver maximum energy per slump to the lower head. Conversely, early and frequent side-wall failures would deliver minimized energy per slump to the lower head.

Maximum energy per slump results from an open crucible, where the top crust is permeable to incoming debris, with no pressure differential from system pressure, and the side-wall is optimized for a single delayed failure (minimum stress, maximum strength). Debris accumulates in the molten pool as the accident progresses, until the pool reaches a maximum size which the side-wall can no longer support. The resultant single slump, late in the accident sequence, delivers maximum energy to the lower head. 
Minimum energy per slump results from an impermeable dome-topped crucible. If the top crust assumes a dome configuration, the molten pool size is minimized because debris is not added to the pool as the accident progresses. With a dome top, molten debris slumps from the pool when the dome fails, falls into the molten pool, and displaces liquid, or when the side-wall fails. In addition, if the side-walls are impenetrable, changes in system pressure cause relatively large pressure differentials, resulting in early and frequent failures. Minimum energy per slump results from a delayed dome top failure combined with early and frequent side-wall failures (maximum stress, minimum strength).

Table 4-1 lists the major assumptions for the bounding cases to calculate pressure stresses in the top crust, and Table 4-2 lists the same for the side crust.

Table 4-1. Bounding cases for structural analysis in the top crust.

\begin{tabular}{lll}
\hline & Maximum Energy & Minimum Energy \\
\hline Permeability & Open crucible & Dome top closed \\
& (permeable top) & crucible (impermeable) \\
Thickness $\left(\mathrm{t}_{\mathrm{d}}\right)$ & Not applicable & $\mathrm{t}_{\mathrm{d}}=\mathrm{t}_{\mathrm{d}}+\Delta \mathrm{t}_{\mathrm{d}}$ \\
Dome height $(\eta)$ & Not applicable & $\eta=\eta+\Delta \eta$ \\
Stress concentration & Not applicable & $\mathrm{K}=\mathrm{K}_{\min }=1.0$ \\
Ulimate strength $\left(\mathrm{S}_{\mathrm{u}}\right)$ & Not applicable & $\mathrm{S}_{\mathrm{u}}=\mathrm{S}_{\mathrm{u}}+\Delta \mathrm{S}_{\mathrm{u}}$ \\
\hline
\end{tabular}

a. Note: $\pm \Delta$ represents uncertainty in the parameter.

Table 4-2. Bounding cases for structural analysis in the side crust.

\begin{tabular}{lll} 
& \multicolumn{1}{c}{ Maximum Energy } & \multicolumn{1}{c}{ Minimum Energy } \\
\hline Thickness $\left(\mathrm{t}_{\mathrm{c}}\right)$ & $\mathrm{t}_{\mathrm{c}}=\mathrm{t}_{\mathrm{c}}+\Delta \mathrm{t}_{\mathrm{c}}$ & $\mathrm{t}_{\mathrm{c}}=\mathrm{t}_{\mathrm{c}}-\Delta \mathrm{t}_{\mathrm{c}}^{\mathrm{a}}$ \\
Stress concentration & $\mathrm{K}=\mathrm{K}_{\min }=1.0$ & $\mathrm{~K}=\mathrm{K}_{\min }=3.1$ \\
Ultimate strength $\left(\mathrm{S}_{\mathrm{u}}\right)$ & $\mathrm{S}_{\mathrm{u}}=\mathrm{S}_{\mathrm{u}}+\Delta \mathrm{S}_{\mathrm{u}}$ & $\mathrm{S}_{\mathrm{u}}=\mathrm{S}_{\mathrm{u}}-\Delta \mathrm{S}_{\mathrm{u}}$ \\
\hline
\end{tabular}

a. Note: $\pm \Delta$ represents uncertainty in the parameter.

Once the crucible side-wall reaches the baffle plate surrounding the core, it may (or may not) receive support from the baffle plate. Minimum energy per slump assumes that the crucible side-wall is weakened by chemical interactions with the baffle plate and fails on contact. Maximum energy per slump assumes that the crucible side-wall receives support from the baffle plate (i.e., it cannot fail) until the baffle plate reaches $1470 \mathrm{~K}$ (temperature where ultimate strength of stainless steel approaches zero). Once the baffle plate has zero strength, it does not support the side-wall of the crucible. 


\subsubsection{Assumptions and Input}

Structural analysis requires information on the material properties, geometry, boundary conditions, loads, and stress concentrations to predict failure. Failure predictions by ultimate strength are used in this analysis. Figure 4-1 shows experimental ultimate strength as a function of temperature, currently documented in MATPRO. The data fit lines are approximate and for use in the parametric studies of this analysis only. Given the large scatter in the ultimate strength data, a detailed stress analysis would be inappropriate, so simple closed-form solutions are proposed.

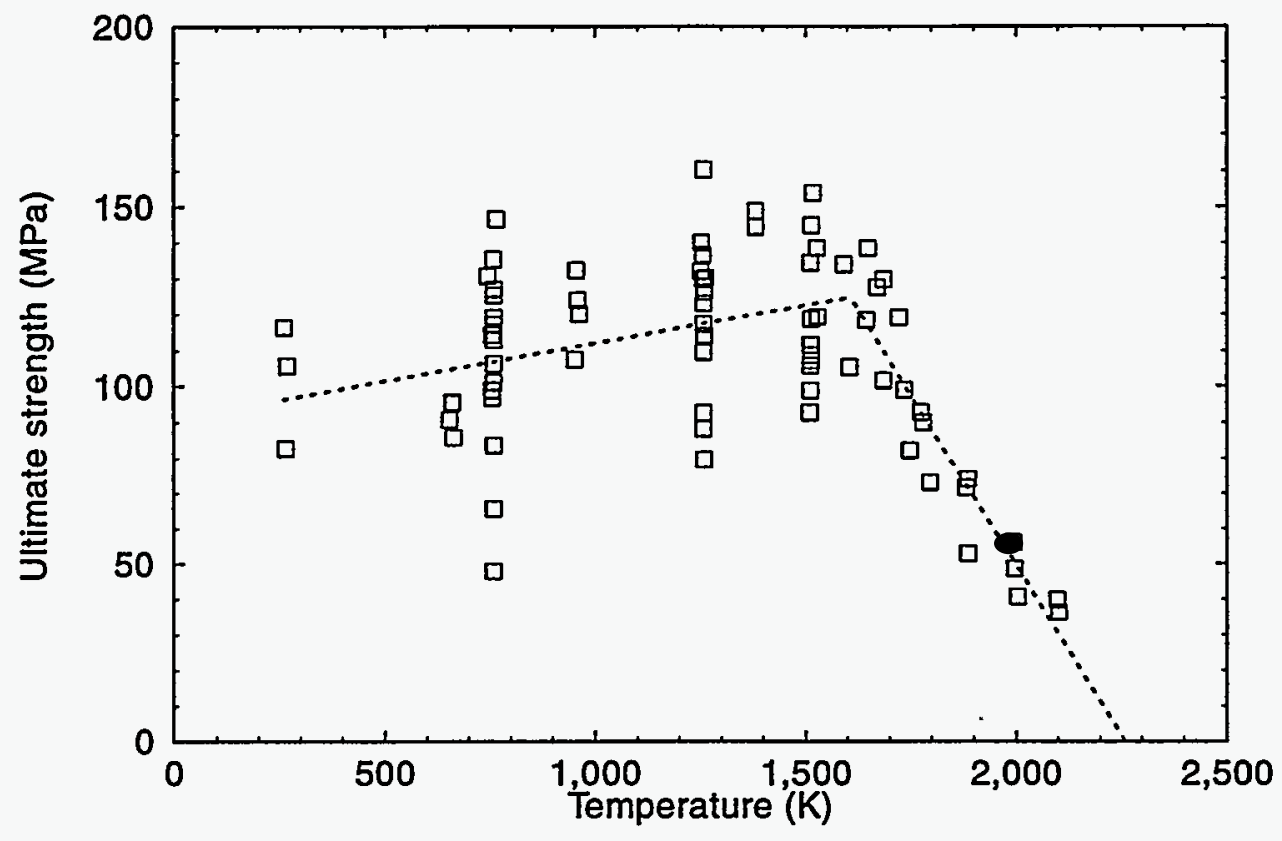

M294-BDR-1094-001

Figure 4-1. Temperature-dependent ultimate strength of $\mathrm{UO}_{2}$

Simplified geometry and boundary conditions help to define closed-form governing equations. The cylindrical side with dome top or open top geometry has been discussed in the previous section. Because the crucible dome and walls are expected to be thin with respect to the cylindrical radius, simple membrane analyses are used; radial stresses are neglected. The crust thickness used in stress calculations is called the "load carrying thickness." It is defined as the portion of the crust which has an ultimate strength greater than zero ( $\mathrm{T}>2251 \mathrm{~K}$, approximately). The simplest, reasonable boundary conditions were specified for the domed top crust and the crust side-walls. The boundary condition for the dome is a simple support tangential to the dome. The boundary condition for the cylindrical sides is a hinged boundary.

Loads, including system pressure differential (inside to outside of impermeable crucible), pressure head from the molten material and dead weight supported by the dome top, will be calculated by the code. The weight of the side wall itself is neglected. 
The stress concentration factor serves to minimize or maximize stress. In the case of porosity, the stress concentrator may take the form of an interior cavity or a surface notch. As stated previously, failure due to propagation of a sharp crack is not directly considered. It is plausible to say that the crust material is ductile at the temperatures of interest, therefore, the stress concentration around the pore causes some plastic deformation near the pore surface, which redistributes stresses around the pore, averting failure. This minimizes stress and the stress concentration factor would be 1.0. On the other hand, stress could be maximized by using the stress concentration around a surface notch and predicting failure when maximum stresses exceed ultimate strength. A surface notch produces a stress concentration factor of approximately 3 which is higher than the concentration around an interior spherical cavity alone or due to the interaction of two fairly closely spaced spherical cavities $(\mathrm{L} / \mathrm{d}=0.5$, where $\mathrm{L}$ is the ligament between cavities and $\mathrm{d}$ is the cavity diameter). ${ }^{4-74,4-75}$

The models calculate effective stresses from pressure loads, and failure (slumping) is predicted when the effective stress exceeds the ultimate strength. The effective stress calculation is only valid up to the yield strength, however, it is used here as a best approximation for this simple analysis. Table 4-3 summarizes the structural input for the top crust, and Table 4-4 lists the same for the side crust.

Table 4-3. Input for analysis of pressure stresses for the top crust.

\begin{tabular}{ll}
\hline Geometry & Dome \\
Loads & $\begin{array}{l}\text { Pressure differential and dead weight on top } \\
\text { of dome (evenly distributed) }\end{array}$ \\
Boundary conditions & Simple support tangential to dome \\
\hline
\end{tabular}

Table 4-4. Input for analysis of pressure stresses for the side crust.

\begin{tabular}{ll}
\hline Geometry & Cylinder \\
Loads & $\begin{array}{l}\text { Pressure differential, molten pool pressure } \\
\text { head, and dead weight on top of dome (evenly } \\
\text { distributed) }\end{array}$
\end{tabular}

Boundary conditions Hinged for pressure stresses

\subsubsection{Governing Equations for Pressure Stresses}

Pressure stresses for both the dome top and the cylindrical side-wall are calculated using closedform, membrane solutions. Mises effective stress is compared to the temperature-dependent ultimate strength to predict failure.

4.3.3.1 Dome Top Crust Pressure Stresses. For the loads and boundary conditions listed in Table 4-3 and Table 4-4, biaxial dome stresses reach a maximum at the center, where they are equal. ${ }^{4-13}$ Debris is assumed to distribute uniformly over the dome. 
$\sigma_{\theta d}=\sigma_{t d}=\left(\frac{r}{2 t_{d}}\right)\left(P+\frac{W_{D}}{A_{d}}\right)$

where

$$
\begin{aligned}
& \sigma_{\theta \mathrm{d}} \quad=\quad \text { tangential stresses for the dome } \\
& \sigma_{\mathrm{td}} \quad=\quad \text { tangential stresses for the dome } \\
& \text { r }=\text { the radius of curvature for the dome } \\
& t_{d}=\quad \text { the dome load bearing thickness at the center. Thickness is measured between } \\
& \text { the radial location where the crust has zero ultimate strength (approximately } \\
& 2251 \mathrm{~K} \text { ) and the outer wall surface. } \\
& \mathrm{P} \quad=\quad \text { the pressure differential due to system pressure (positive pressure outward) } \\
& \mathrm{W}_{\mathrm{D}} \quad=\quad \text { weight of the dome and debris supported by the dome (negative downward) } \\
& \mathrm{A}_{\mathrm{d}} \quad=\quad \text { surface area of the dome. }
\end{aligned}
$$

The dome radius ( $r)$ geometrically relates to the crucible radius $(R)$ and center dome height ( $\eta$ ), Figure 4-2.

$r=\frac{1}{2}\left(\frac{R^{2}}{\eta}+\eta\right)$

Substituting into Equation (4-46):

$$
\sigma_{\theta d}=\frac{1}{4}\left[\frac{R}{t_{d}} \frac{R}{\eta}+\frac{\eta}{t_{d}}\right]\left(P+\frac{W_{D}}{A_{d}}\right)
$$

The $\eta / t_{d}$ term is generally negligible compared to the product of $R / t_{d}$ and $R / \eta$. Hence stress is approximately inversely proportional to the thickness.

The Mises effective stress $\left(\sigma_{\mathrm{E}}\right)$ is equal to the hoop stress.

$$
\sigma_{\mathrm{E}}=\sigma_{\theta \mathrm{d}} .
$$




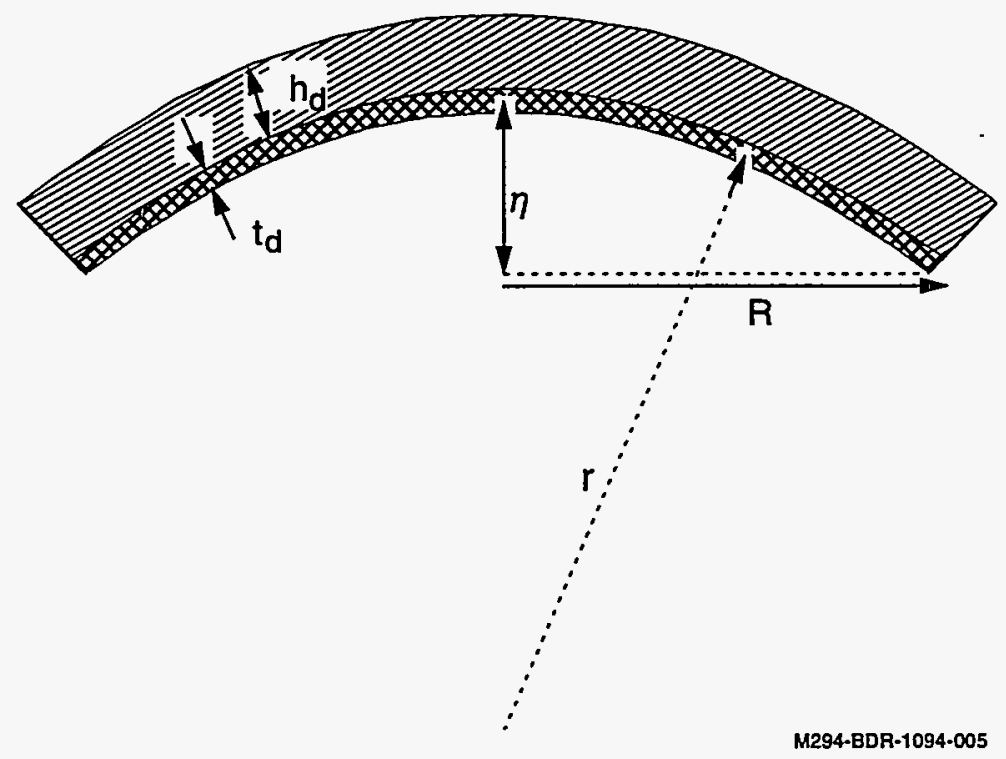

Figure 4-2. Schematic of top crust dome geometry.

The effective stress is compared to temperature-dependent ultimate strength $\left(S_{u}\right)$ and failure is predicted if

$\mathrm{K} \sigma_{\mathrm{E}}>\mathrm{S}_{\mathrm{u}}(\mathrm{T})$

4.3.3.2 Crust Side-Wall Pressure Stresses. Failure in the crust side-wall results from a combination of high stress and high temperature. Side-wall hoop stresses increase approximately linearly from the top of the crucible, peaking near the bottom; however, temperatures are highest near the top. Sidewall stresses near the top and bottom of the crucible are influenced by the boundary conditions imposed by the ends, which are approximated as hinged.

The following equations are used to calculate the side-wall hoop stresses for the crucible. Hoop stress is calculated as a function of distance $(\mathrm{z})$ from the bottom of the crucible. ${ }^{4-13}$

Closed crucible, lower half $(0 \leq \mathrm{z} \leq \mathrm{H} / 2)$ :

$\sigma_{\theta B}=\frac{R}{t_{c}}[P+\gamma(H-z)]-\frac{R}{t_{c}}\left[P+\gamma H+\frac{W_{D}}{A_{d}}\right] e^{-\lambda z} \cos (\lambda z)$

Closed crucible, upper half $(\mathrm{H} / 2<\mathrm{z} \leq \mathrm{H})$ : 


$$
\sigma_{\theta T}=\frac{R}{t_{c}}[P+\gamma(H-z)]-\frac{R}{t_{c}}\left[P+\frac{W_{D}}{A_{d}}\right] e^{-\lambda(H-z)} \cos [\lambda(H-z)]
$$

Open crucible, full length $(0 \leq \mathrm{z} \leq \mathrm{H})$ :

$$
\sigma_{\theta}=\frac{R \gamma}{t_{c}}\left\{H\left[1-e^{-\lambda z} \cos (\lambda z)\right]-z\right\}
$$

where

$$
\begin{aligned}
& \sigma_{\theta}=\text { the hoop stress, additional subscripts } \mathrm{B} \text { and } \mathrm{T} \text { indicate bottom and top halves, } \\
& \text { respectively } \\
& \mathrm{R} \quad=\quad \text { the crucible radius } \\
& t_{c}=\quad \text { the crust side-wall load bearing thickness at axial location } \mathrm{z} \text {. Thickness is } \\
& \text { measured between the radial location where the crust has zero ultimate strength } \\
& \text { (approximately } 2251 \mathrm{~K} \text { ) and the outer wall surface. } \\
& \mathrm{P} \quad=\quad \text { the pressure differential due to system pressure (positive outward) } \\
& \gamma \quad=\quad \text { the specific weight of the molten pool } \\
& \mathrm{H}=\text { the height of the crucible (assumed equal to height of molten pool) } \\
& \mathrm{W}_{\mathrm{D}} \quad=\quad \text { weight } \text { of the dome and debris supported by the dome } \\
& \mathrm{A}_{\mathrm{d}} \quad=\quad \text { surface area of the dome. } \\
& \mathrm{z}=\text { the distance from the bottom of the crucible to the point of interest. } \\
& \lambda=\left[\frac{3\left(1-v^{2}\right)}{R^{2} t_{c}^{2}}\right]^{1 / 4}
\end{aligned}
$$

where $v$ is Poisson's ratio.

Note that if the crucible side-wall or top is partially permeable, the system pressure differential may equilibrate over time so that only sudden changes in system pressure need to be considered.

Figure 4-3 shows the hoop stress for a closed crucible of typical dimensions and loads $(K=3, R=1.5$ $\mathrm{m}, \mathrm{t}_{\mathrm{c}}=0.03 \mathrm{~m}, \mathrm{~h}=3.0 \mathrm{~m}, \mathrm{P}=316 \mathrm{~Pa}, \gamma=80.36 \mathrm{~N} / \mathrm{m}^{3}, \mathrm{~W}=40,000 \mathrm{~N}$ ). The pressure differential $(\mathrm{P})$ equals 
the partial pressure of urania at $4000 \mathrm{~K}$. Note that for most of the length of the crucible, system pressure differential and pressure head dominate hoop stresses $\left(\sigma_{\theta \mathrm{P}}\right)$ and end effects are negligible. Hoop stress is well-described by:

$\sigma_{\theta P}=\frac{R}{t_{c}}[P+\gamma(H-z)]$

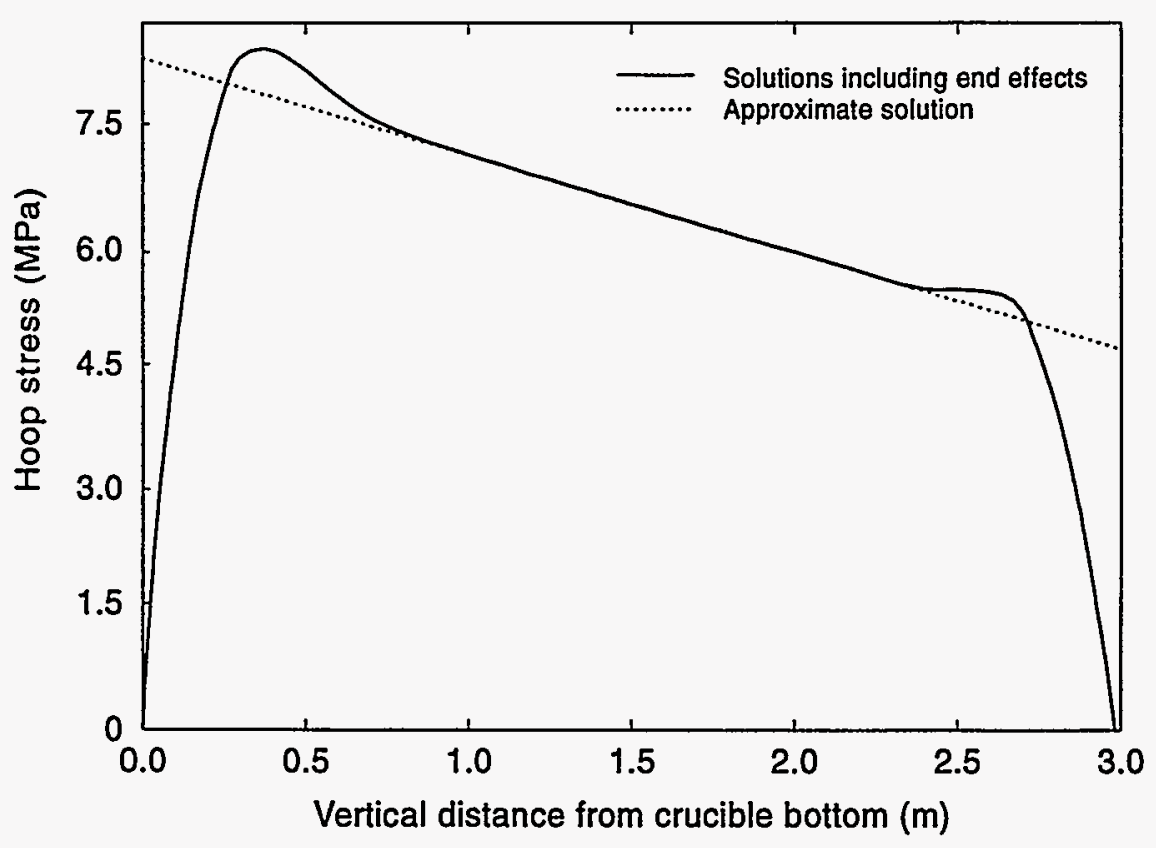

Figure 4-3. Comparison of approximate solution and solution with end effects for side-wall hoop stresses in a closed cylinder.

End effects from the top and bottom boundaries bring hoop stresses to zero at the ends, but create stresses slightly higher than those predicted from the above equation near the ends. The stresses at the "humps" near the ends in Figure 4-3 are within 10\% of the stresses calculated at the ends using the above equation.

The closed crucible side-wall supports the dead weight of the dome and debris lying on the dome $\left(W_{D}\right.$, a negative number), resulting in compressive axial stress which is combined with the axial stress from the system pressure differential.

Closed crucible:

$\sigma_{z}=\left[\frac{P R}{2 t_{c}}+\frac{W_{D}}{2 \pi R t_{c}}\right]$ 
The open crucible supports no axial load except the weight of the side-wall itself, which is considered negligible.

Open crucible:

$\sigma_{\mathrm{z}}=0$

The Mises effective stress $\left(\sigma_{\mathrm{E}}\right)$ is:

$\sigma_{\mathrm{E}}=\left(\sigma_{\theta}^{2}-\sigma_{\mathrm{z}} \sigma_{\theta}+\sigma_{\mathrm{z}}^{2}\right)^{1 / 2}$

where, $\sigma_{\theta}$ is $\sigma_{\theta \mathrm{B}}, \sigma_{\theta \mathrm{T}}$ or $\sigma_{\theta}$, from Equations (4-51), (4-52), and (4-53). $\sigma_{\mathrm{z}}$ is from Equations (4-56) and (457).

Failure is predicted for any axial location on the side-wall using Equation (4-50):

$\mathrm{K} \sigma_{\mathrm{E}} \geq \mathrm{S}_{\mathrm{u}}(\mathrm{T})$

$\mathrm{S}_{\mathrm{u}}$ depends on the average temperature at the axial location of interest.

4.3.3.3 Summary of Governing Equations. The following equations are used to predict top and side-wall crust failure.

From Equation (4-50), failure is predicted for any case when:

$\mathrm{K} \sigma_{\mathrm{E}} \geq \mathrm{S}_{\mathrm{u}}(\mathrm{T})$

From Equations (4-48) and (4-49), the closed crucible, dome top, Mises effective stress:

$\sigma_{E}=\frac{1}{4}\left[\frac{R}{t_{d}} \frac{R}{\eta}+\frac{\eta}{t_{d}}\right]\left(P+\frac{W_{D}}{A_{d}}\right)$

Closed crucible, side-wall, Mises effective stresses, lower (bottom) and upper (top) halves:

$$
\begin{aligned}
& \sigma_{\mathrm{EB}}=\left(\sigma_{\theta \mathrm{B}}^{2}-\sigma_{\mathrm{z}} \sigma_{\theta \mathrm{B}}+\sigma_{\mathrm{z}}^{2}\right)^{1 / 2} \\
& \sigma_{\mathrm{ET}}=\left(\sigma_{\theta \mathrm{T}}{ }^{2}-\sigma_{\mathrm{z}} \sigma_{\theta \mathrm{T}}+\sigma_{\mathrm{z}}^{2}\right)^{1 / 2} .
\end{aligned}
$$

From Equation (4-51), closed crucible, side-wall, hoop stress, lower half $(0 \leq \mathrm{z} \leq \mathrm{H} / 2)$ : 


$$
\sigma_{\theta B}=\frac{R}{t_{c}}[P+\gamma(H-z)]-\frac{R}{t_{c}}\left[P+\gamma H+\frac{W_{D}}{A_{d}}\right] e^{-\lambda z} \cos (\lambda z)
$$

From Equations (4-52) and (4-54), closed crucible, side-wall, hoop stress, upper half $(H / 2<\mathrm{z} \leq \mathrm{H})$ :

$$
\sigma_{\theta T}=\frac{R}{t_{c}}[P+\gamma(H-z)]-\frac{R}{t_{c}}\left[P+\frac{W_{D}}{A_{d}}\right] e^{-\lambda(H-z)} \cos [\lambda(H-z)]
$$

where

$$
\lambda=\left[\frac{3\left(1-v^{2}\right)}{R^{2} t_{c}^{2}}\right]^{1 / 4} .
$$

From Equation (4-56), closed crucible, side-wall, axial stress:

$\sigma_{z}=\left[\frac{P R}{2 t_{c}}+\frac{W}{2 \pi R t_{c}}\right]$

From Equations (4-53), (4-54),(4-57), and (4-58), open crucible, side-wall, Mises stress $(0 \leq \mathrm{z} \leq \mathrm{H})$ :

$$
\sigma_{E}=\frac{R \gamma}{t_{c}}\left\{H\left[1-e^{-\lambda z} \cos (\lambda z)\right]-z\right\}
$$

where

$$
\lambda=\left[\frac{3\left(1-v^{2}\right)}{R^{2} t_{c}^{2}}\right]^{1 / 4} .
$$

\subsection{Impact of Proposed Modifications}

The proposed model changes will have the most significant impact on plant calculations where, like TMI-2, the core is partially or totally reflooded during molten pool formation and crust failure is likely. For these conditions, the current thermally-based models cannot predict the location nor the timing of crust failure without user intervention ${ }^{a}$. That is, the crust will fail only if the molten pool grows to the bottom of the core or the user specifies that the crust fails when the molten pool spreads into the outer assembly. For the TMI-2 calculations, this combination either resulted in no failure at all because the outer assemblies remained relatively cool following the reflooding of the core or bottom failure of the molten pool for an extreme core uncovery scenario. In a general case where the molten pool is covered by water, crust failure will only occur using the current thermally-based models if the equilibrium crust thickness, determined from the balance of local heat transfer from the molten pool and external cooling is less than the default

a. In early SCDAP/RELAP5/MOD2.5 TMI-2 calculations crust failure was predicted due to the thinning of the metallic layer supporting the ceramic debris and molten pool. Results from the experiments such as MP2 , a better understanding of the failure location and conditions, and structural analyses such as those presented in this report now clearly discount metallic layer thinning as the correct failure mechanism. 
thickness originally derived for TMI-2 conditions. As discussed in the proceeding sections, a combination of structural and thermally based models should be better able to predict both location and timing of failure because the strength of the crust and loading applied to the crust are considered. In the specific case of TMI-2, as shown in the demonstration calculations in the following section, it is likely that the failure of both the top and side crust would be predicted under the conditions occurring in the accident if the structural aspects of the crust failure are considered, even though there are relatively large uncertainties in the actual crust thickness and material properties. In the general case, these new models will be able to account for the likely changes in crust failure thicknesses due to (a) rapid pressure fluctuations, which increases the loading on the crust and (b) the height of the molten pool.

The proposed modeling changes will have a less significant impact on plant transients where the molten pool is completely uncovered. In plant transients where molten pool growth occurred in superheated steam, the molten pool grew primarily because intact or degraded assemblies adjacent to the molten pool were heating rapidly to ceramic melting temperatures. Molten pool slumping occurred because the outer assemblies reached ceramic melting temperatures, not because the molten pool spread radially into relative cold outer assemblies. However, the proposed model improvements will still have an impact because they will allow a better prediction of the elevation of the molten pool crust failure, and the subsequent mass of the slumping material. In the current models, it is assumed that the entire mass of the molten pool slumps when the any portion of the outer assemblies become molten. With the new models, the slumping of the molten pool could occur more gradually if either the top crust or upper portion of the side crust failed first or more massively if the lower portion of the side crust failed first.

Although the specific impact of the new models will not be determined until the developmental assessment of these models is completed, the incorporation of more physically based models should have a strong impact in reducing some of the current limitations observed in the current models and in eliminating the need for the user to force slumping to occur. As shown in the parameter calculations contained in the next few sub-sections, the addition of these new models could be very beneficial.

The intent of the following preliminary parametric studies is to show the importance of added parameters in the proposed models. Comparisons are made between crust failure predicted by current code models, failure predicted by the proposed models, and the TMI- 2 crust failure estimates. In the TMI- 2 accident, a non-homogeneous, primarily ceramic crucible encased a region of molten debris in the core region (Figure 4-4). Because crust permeability is unknown, both open and closed crucible failure predictions are examined. TMI-2 accident data and analyses indicate that the crucible radius was approximately $146 \mathrm{~cm}, 47$ and the outside crust surface temperature was $1710 \mathrm{~K}$, or less. ${ }^{48}$ The approximate TMI-2 crust total side-wall thickness (though there was great variation, see Figure 4-4) (-6 $^{\text {was }}$ approximately $20 \mathrm{~cm} .{ }^{4-7}$ Assuming a linear temperature distribution, the load bearing thickness is only 7.5 $\mathrm{cm}$, because ultimate strength extrapolates to zero at $2251 \mathrm{~K}$. Note that the current crust model uses only total crust thickness. The failure maps predicted by the proposed models use-simplified governing equations and simplified ultimate strength-to-temperature relations, but are adequate for these comparisons. The failure criterion is based on ultimate strength, so a description of the temperaturedependent ultimate strength relationships precedes the failure comparisons. 


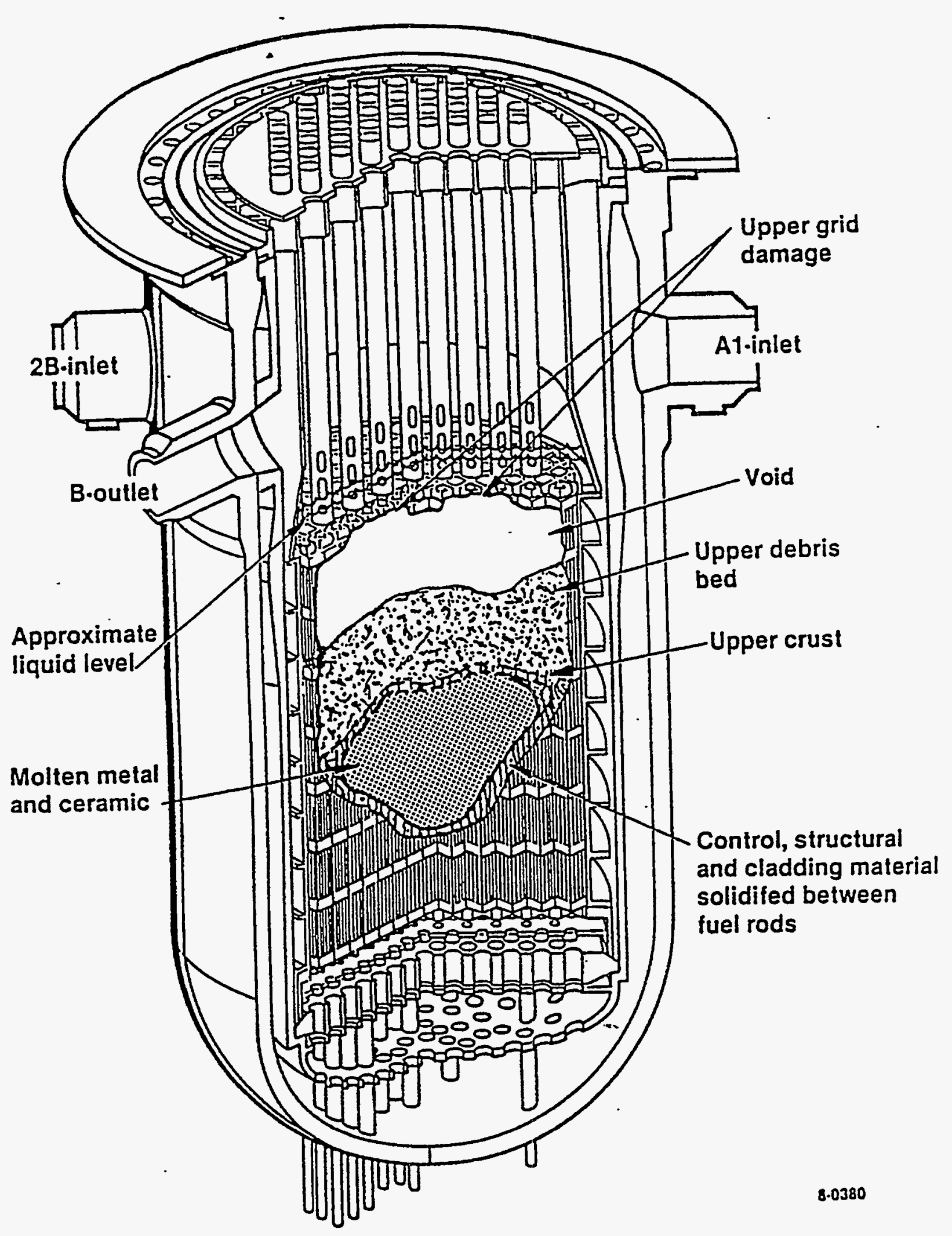

Figure 4-4. Schematic of TMI-2 endstate 


\subsubsection{Temperature-Dependent Ultimate Strength}

Ultimate strength of $\mathrm{UO}_{2}$ is dependent on temperature, porosity, pore size, strain rate and, to a lesser degree, grain size. Because most of these parameters are not quantified, simplified temperature-dependent ultimate strengths from MATPRO data (Figure 4-1) are estimated.

$S_{\mathrm{u}}=0.020876 \mathrm{~T}+92.233600 \mathrm{~K}<\mathrm{T} \leq 1609 \mathrm{~K}$

$\mathrm{S}_{\mathrm{u}}=-0.19598 \mathrm{~T}+441.221609 \mathrm{~K}<\mathrm{T}<2251 \mathrm{~K}$

$\mathrm{S}_{\mathrm{u}}=0.0 \mathrm{~T} \geq 2251 \mathrm{~K}$

where $S_{u}$ is ultimate strength (MPa) and T is temperature (K). Equations (4-61) through (4-63) can be used to define average ultimate strength in terms of the outer wall temperature, if the inner side-wall temperature is assumed to be the liquidus temperature of $\mathrm{UO}_{2}(2873 \mathrm{~K})$ and the temperature distribution is assumed linear.

\subsubsection{Crust Side-Wall}

As shown in Figure 4-3 and discussed in the previous section, Equation (4-55) describes the hoop stresses in the side-wall for most of the length of the wall. It is simple and adequate for these parametric studies. Equations (4-55) through (4-58) define the effective stress in terms of pressure differential and the radius-to-thickness ratio.

Substituting results from Equations (4-55) through (4-58) into the left side of Equation (4-50), and substituting Equations (4-61) and (4-62) into the right side of Equation (4-50), gives the following relationship.

$\sigma_{\mathrm{E}}\left(\mathrm{P}, \gamma(\mathrm{H}-\mathrm{z}), \mathrm{r} / \mathrm{t}_{\mathrm{c}}\right) \geq \mathrm{S}_{\mathrm{u}}\left(\mathrm{T}_{\text {outer }}\right)$

Effective stress is a function of the stress concentration factor, system pressure and pressure head differentials, and the radius-to-thickness ratio. Average ultimate strength is a function of the outer sidewall temperature, assuming a linear temperature distribution from a molten inside to cooled outside. Equations (4-55) through (4-63) and Equation (4-50) are used to construct the failure maps in this section. Note that the outer wall temperature changes (and thus, ultimate strength) with axial location, therefore the height of crust failure point can be identified and the molten pool can be drained accordingly.

Open crucible:

The open crucible has very low stresses because the dominant load, pressure head, is low. Figure 4-5 plots the failure lines for molten pool height as a function of side-wall thickness for various outside sidewall temperatures. To construct this failure map, maximum possible pool height is assumed to be approximately the length of the core $(3 \mathrm{~m})$ and crucible radius is taken from TMI-2 $(146 \mathrm{~cm})$. Results from 
the failure map show that for TMI- 2 conditions of $1710 \mathrm{~K}$ outside wall temperature and $1.3 \mathrm{~m}$ molten pool height, the side-wall would have to thin to $3 \mathrm{~mm}$ before failure would be predicted. This is inconsistent with TMI-2 data which indicates that the load bearing wall thickness was approximately $7.5 \mathrm{~cm}$. Alternatively, for TMI-2 conditions of $7.5 \mathrm{~cm}$ side-wall (load bearing) thickness and $1.3 \mathrm{~m}$ molten pool height, the predicted outside wall temperature at failure greater than $2200 \mathrm{~K}$. TMI-2 analysis indicates the molten pool slumped with an outside wall temperature of $1710 \mathrm{~K}$ (or less). The current code would not predict failure until the side-wall reached melt (more than $3000 \mathrm{~K}$ ). Clearly, though the open crucible model makes a better prediction than the current model, neither match the TMI-2 data.

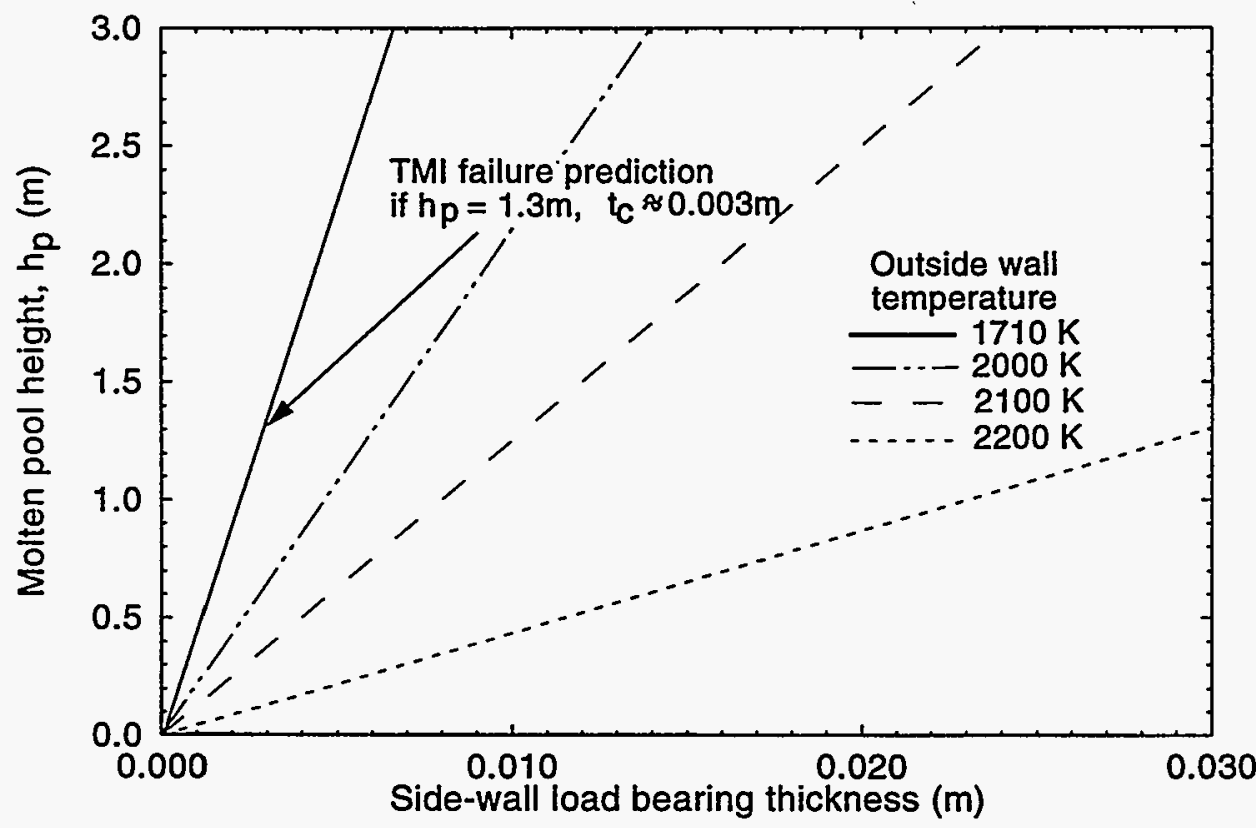

M294-BDR-1094-008

Figure 4-5. Side-wall failure map for a open crucible with molten interior and linear through-wall temperature distribution.

\section{Closed crucible:}

The closed crucible, if partially or totally gas impermeable, is subjected to relatively high stresses when the system pressure undergoes modest fluctuations. For the impermeable case, the thickness at failure will be much larger, or the outside wall temperature will be lower, than in the permeable (open) crucible. Figure 4-6 plots failure lines for system pressure differential as a function of side-wall thickness for different outer wall temperatures. Results from this map show that TMI- 2 conditions $(7.5 \mathrm{~cm}$ thick load bearing wall, with an outside wall temperature of $1710 \mathrm{~K}$ ) would be expected to fail with a pressure differential of approximately $1.1 \mathrm{MPa}$, or more. Such a pressure differential could be caused by a sudden change in pressure. Figure 4-7 shows the TMI-2 pressure history, with large pressure fluctuations between 190 and 224 minutes, followed by major relocation between 224 and 226 minutes. The closed crucible model is capable of approximating the TMI-2 accident data; whereas, the current model does not allow the crust to fail until the crust melts.

Open crucible versus closed crucible: 


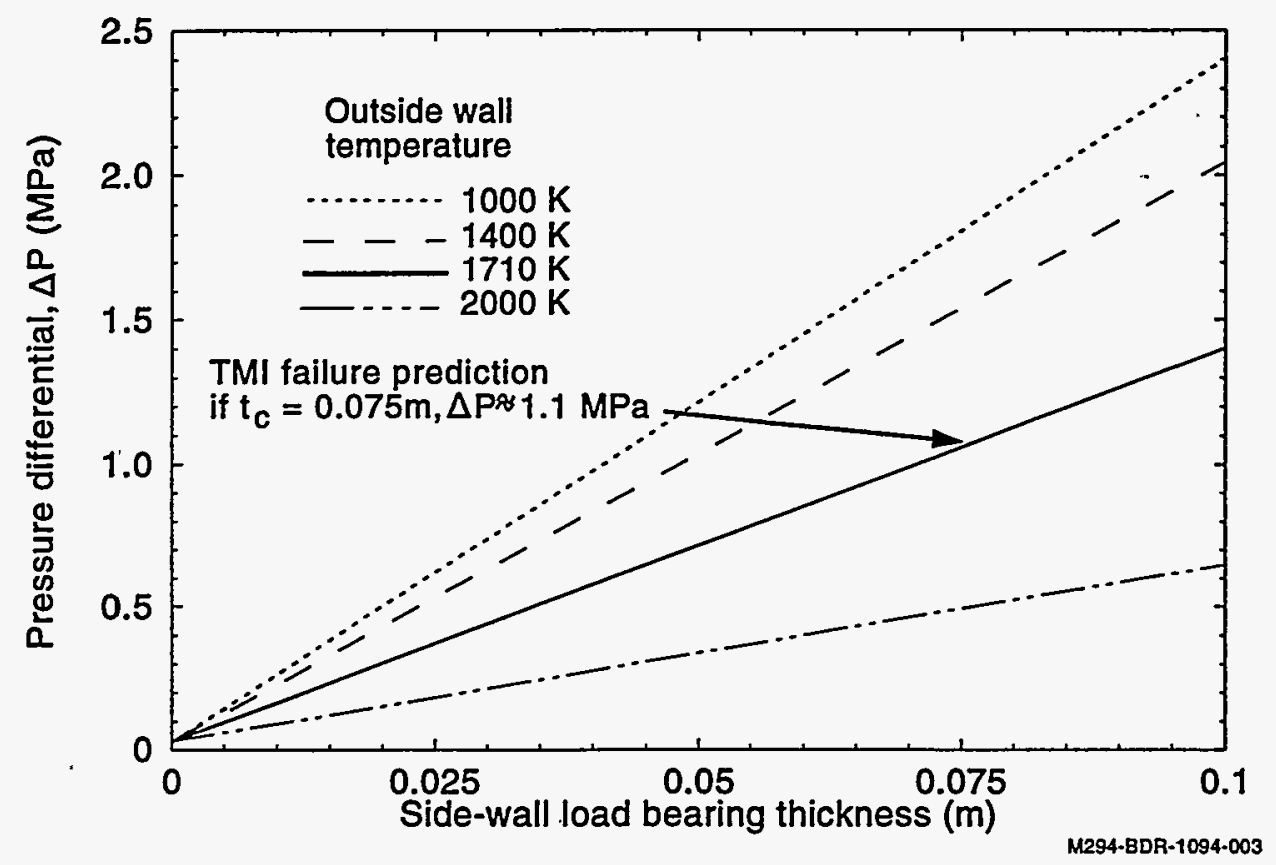

Figure 4-6. Side-wall failure map for a closed crucible with molten interior and linear through-wall temperature distribution.

From the above analyses, it is clear that permeability has an important effect on crust failure. Because the TMI-2 case appears to be one requiring some degree of impermeability does not mean that all crusts will be impermeable; therefore, both bounding cases are still needed. With $1 \mathrm{MPa}$ system pressure and a $3 \mathrm{~m}$ molten pool, permeability (versus impermeability) reduces the thickness at failure by 5; and this factor increases with increasing changes in system pressure. Another parameter which plays an important role in predicting failure is the stress concentration factor accounting for porosity or flaws. The stress concentration accounts for a factor of approximately 3 , independent of pressure. The range for failure thickness or failure pressures of the bounding analyses would be greatly reduced if the crust was known to be permeable. Knowledge of the stress concentration factor due to porosity would also reduce the range.

\subsubsection{Crust Top Dome}

The dome top is assumed to exist in an impermeable closed crucible. Comparing Equations (4-48) and (4-55) reveals that the stresses in the dome top and crucible side both depend on pressure differentials and radius-to-thickness ratios. In addition the dome top includes the height of the dome. Assuming the radius-to-thickness and radius-to-height ratios are much larger than the height-to-thickness ratio (a fairly flat dome), Equation (4-48) can be reduced to:

$\sigma_{\theta D}=\frac{1}{4} \frac{R}{t_{d}} \frac{R}{\eta}\left(P+\frac{W_{D}}{A_{d}}\right)$

Note that $R$ refers to the crucible radius and $\eta$ refers to the dome height at the center (Figure 4-2). 
Endstate examination of the TMI-2 accident indicates that the debris lying on top of the crucible crust accounted for approximately $20 \%$ of the total core weight. ${ }^{4-15,4-16}$ For a domed configuration, the maximum pressure this debris imposes on the dome (less than $0.05 \mathrm{MPa}$ ) is negligible compared to changes in system pressure. Hence the last term in Equation (4-66) can be ignored.

Figure 4-8 plots the change in system pressure versus dome load bearing thickness for various ratios of crucible radius-to-dome height $(\mathrm{R} / \mathrm{\eta})$. This failure map assumes a linear through-wall temperature distribution, varying from inside melt $(3150 \mathrm{~K})$ to an outside temperature of $1710 \mathrm{~K}$, as predicted in TMI2 analysis. ${ }^{48}$ Recall that the proposed models predict side-wall failure with the same temperature distribution and a load bearing thickness of $7.5 \mathrm{~cm}$ at approximately $1.1 \mathrm{MPa}$. The dome height and thickness for TMI-2 are unknown. However, it is clear from Figure 4-8, that a dome, with $R / \eta$ greater than approximately 3.5 and load bearing thickness of $7.5 \mathrm{~cm}$, is expected to fail at pressure differentials less than 1.1 MPa. In other words, assuming the wall temperatures and thicknesses are the same, domes flatter than $R / \eta=3.5$ will fail before the side-wall from changes in system pressure.

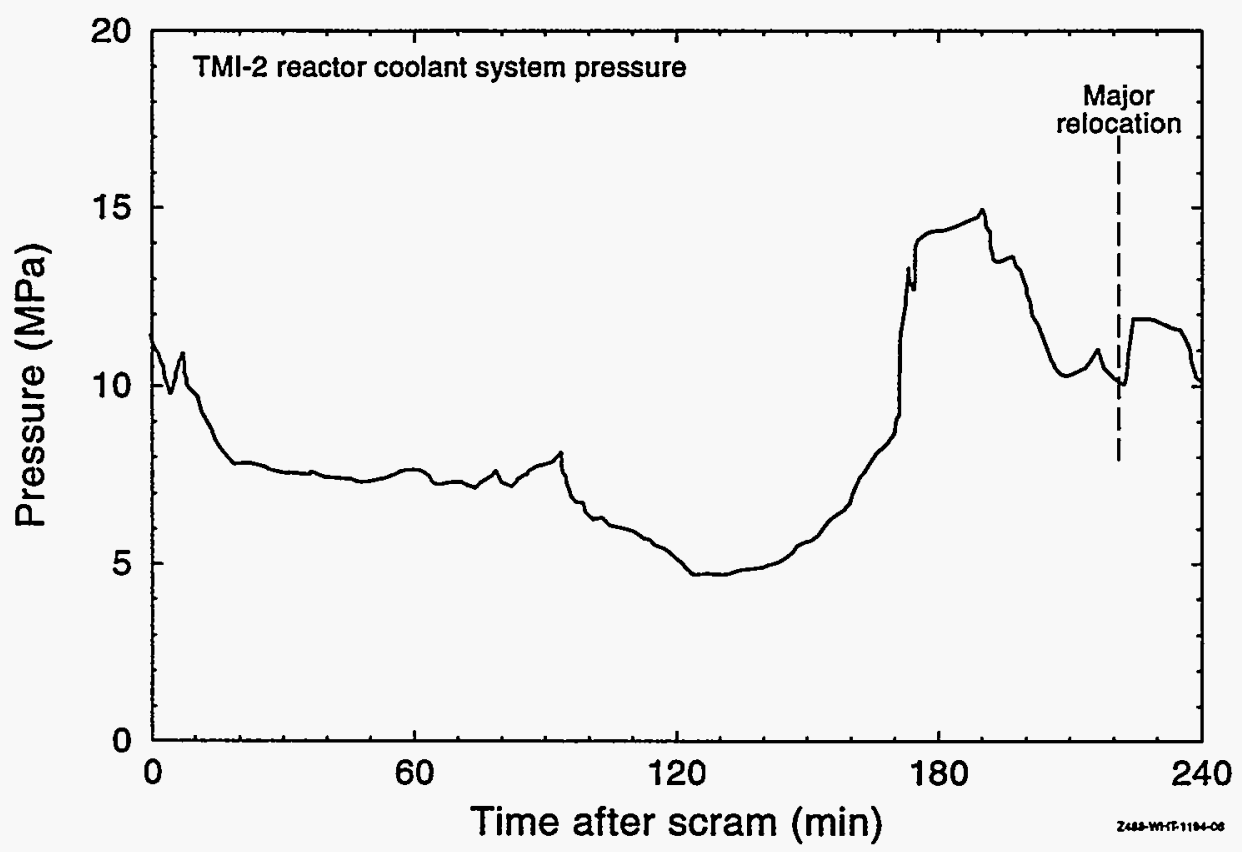

Figure 4-7. TMI-2 accident pressure history.

Note that the partial pressure of urania at $4000 \mathrm{~K}$ is approximately $0.3 \mathrm{MPa}$. For the assumed TNI-2 temperature distribution, the proposed model predicts failure in a fairly flat dome $(R / \eta=10)$ at a load bearing thickness of approximately $6 \mathrm{~cm}$ (total thickness of $17 \mathrm{~cm}$ ). Current code models do not predict top crust failure until the total thickness reduces to $0.5 \mathrm{~cm}$.

Under some conditions, there may be some concern that the dome geometry gives unrealistic stress predictions. For example, when dead weight stresses are significantly greater than stresses from a positive pressure differential, the maximum stress of a domed top crust is compressive (large deformations are not 


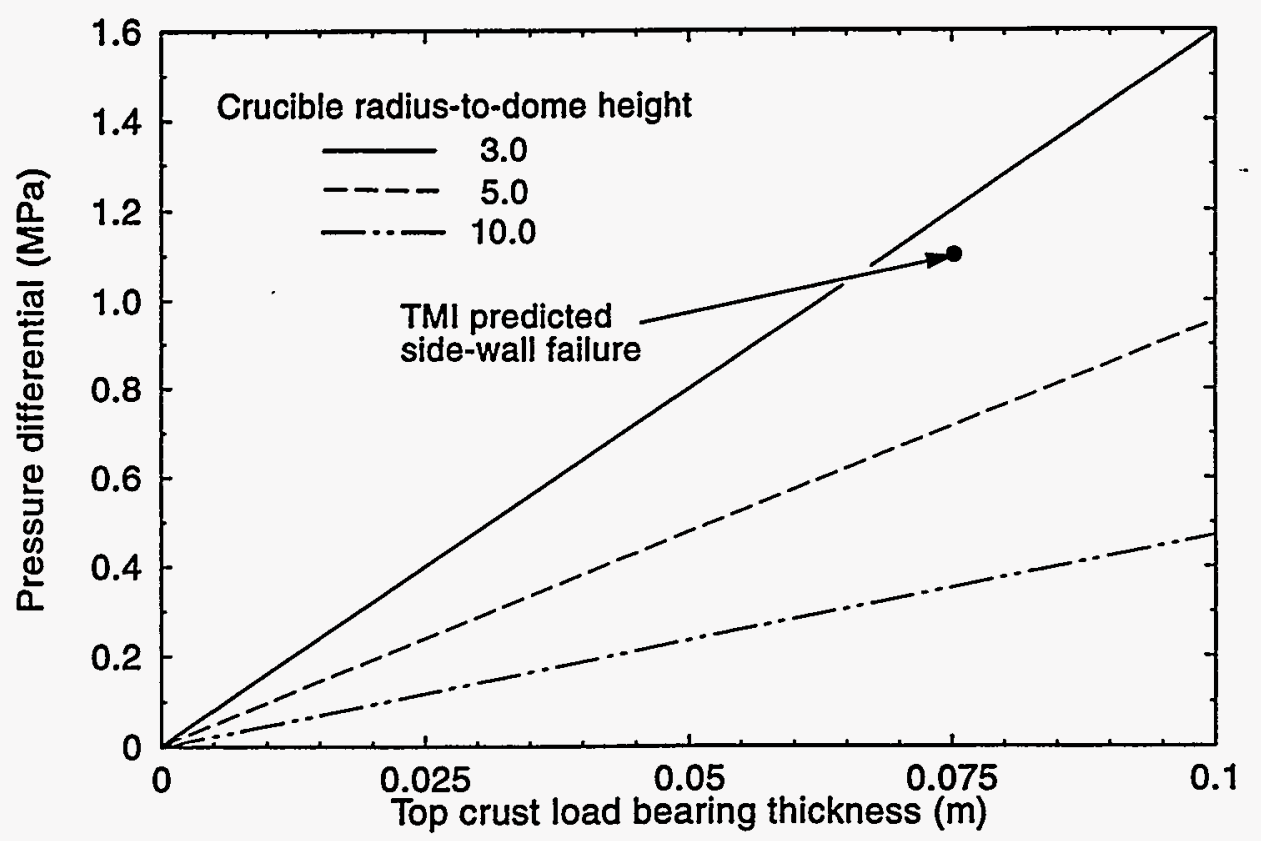

M294-BDR-1194-015

Figure 4-8. Dome top failure map for a closed crucible with molten interior and linear through-wall temperature distribution, assuming $1710 \mathrm{~K}$ outer wall temperature.

accounted for). However, if the top crust were modeled as flat, maximum stresses would be tensile. Figure 4-9 shows a comparison of maximum stresses for a plate solution proposed by Engelstad and Feng ${ }^{4-14}$ and the simple dome solution proposed here. The loading conditions are those posed by Engelstad and Feng: a crust with $5 \mathrm{kPa}$ pressure load on top and a through thickness temperature gradient (the plate solution accounts for thermal bending stresses) Engelstad does not account for porosity, so $\mathrm{K}=1.0$ in this comparison. Although the signs of the stresses are opposite, fortuitously, the magnitudes of these stresses compare very well. The effective stresses, which determine failure and are always positive, will also compare well.

\subsection{Summary and Recommendations}

The proposed model changes will have the most significant impact on plant calculations where, like TMI-2, the core is partially or totally reflooded during molten pool formation and crust failure is likely. For these conditions, the current thermally-based models cannot predict the location nor the timing of crust failure without user intervention. In the general case, these new models will be able to account for the likely changes in crust failure thicknesses due to (a) rapid pressure fluctuations, which increases the loading on the crust and (b) the height of the molten pool. With the new model, slumping of the molten pool could occur more gradually if either the top crust or upper portion of the side crust failed first or more massively if the lower portion of the side crust failed first. Analysis shows that bottom crust failure is very unlikely before side crust failure.

Although this new model represents more of the critical processes associated with crust failure, it must be noted that the precise timing and location of the failure will still have relatively large uncertainties. 


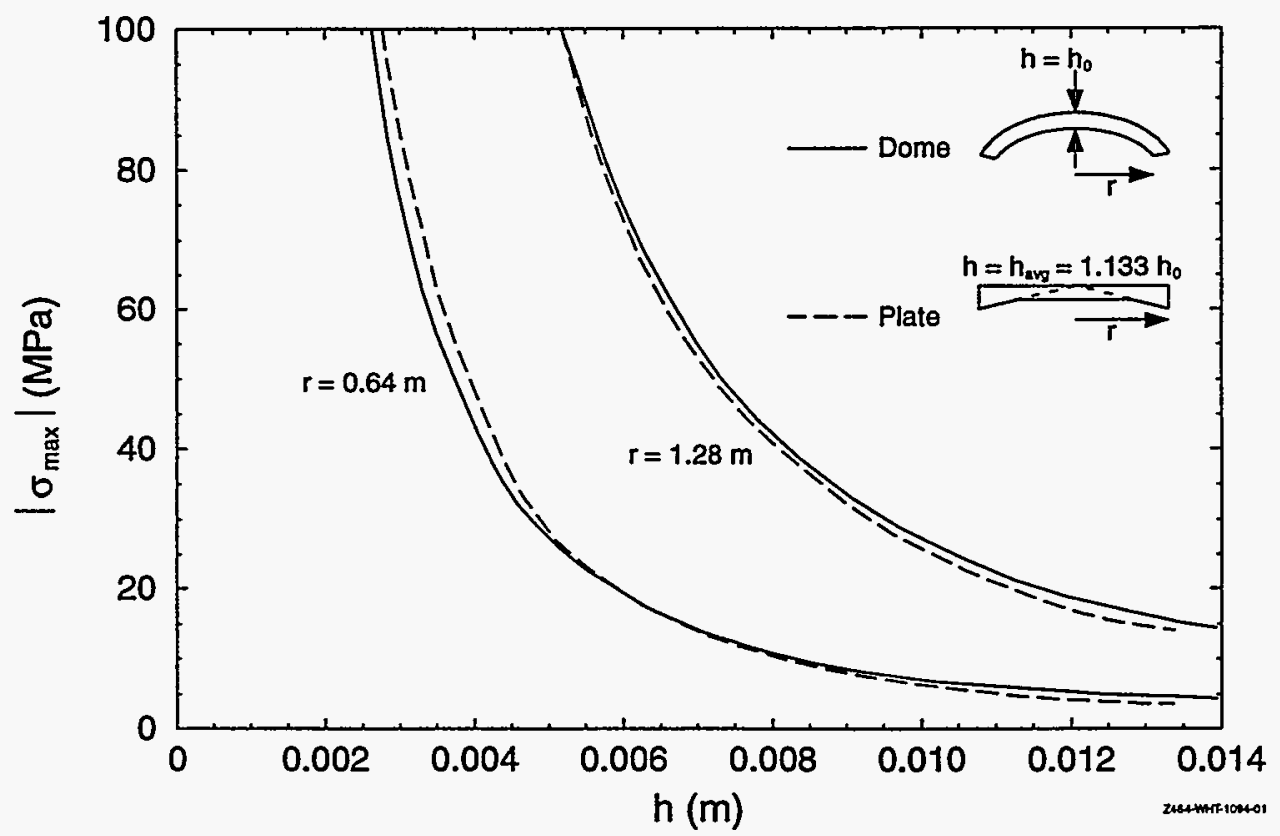

Figure 4-9. Comparison of maximum stress solutions for top crust, modeled with dome geometry and modeled with Engelstad and Feng ${ }^{4-14}$ plate geometry.

Uncertainties stem from limited data for assessment, non-homogeneity of the crust thickness and composition, lack of high temperature mechanical properties for the crust, and possible influence of cracking of the crust if it is exposed to water (as occurred in TMI-2). To reduce uncertainties, crust permeability (or lack of) and the proper stress concentration factor should be investigated, and a more detailed statistical analysis of the temperature-dependent ultimate strength data should be performed, with any available new tests added to the database. To account for the remaining uncertainties, a bounding approach which allows the user to bound the mass or energy of the melt relocating after crust failure is recommended. This bounding approach is also consistent with the bounding approach used in the current slumping models. As a result, the user should be able to quickly establish an upper and lower bound on the melt mass and energy at lower head failure caused by uncertainties in crust failure and melt slumping processes.

\subsection{References}

4-1. E. L. Tolman et al., TMI-2 Core Bore Acquisition Summary Report, EGG-TMI-7385, Rev. 1, February 1987.

4-2. M. Epstein and H. K. Fauske, The Three Mile Island Unit 2 Core Relocation - Heat Transfer and Mechanism, Nuclear Technology, Vol. 87, December 1989, 1021 - 1035.

4-3. P. Kuan and E. L. Tolman, Damaged TMI-2 Core Consolidated Region Crust Behavior, Transactions of the American Nuclear Society, 57, 413 (1988).

4-4. C. Allison, "Results from Core Bore Examinations,"International Atomic Energy Agency Mission, Instituto de Investigaciones Electricas, Cuernavaca, Mexico, May 17-23, 1992.

4-5. R. R Hobbins, M. L. Russell, C. S. Olsen, R. K. McCardell, Molten Material Behavior in the Three Mile Island Unit 2 Accident, Nuclear Technology, Vol. 87, December 1989, 1005 - 1012. 
4-6. M. L. Russell, personal correspondence with S. A. Chavez, October 6, 1994.

4-7. M. L. Russell, TMI-2 Core Horseshoe Ring Examinations, GEND-INF-083, October 1987.

4-8. P. Kuan, Core Relocation in the TMI-2 Accident, EGG-TMI-7402, November 1986.

4-9. C. M. Allison, et al., SCDAP/RELAP5/MOD3.1 Code Manual, Volume I, NUREG/CR-6150, EGG-2720, October 1993.

4-10. P. Kuan, Core Relocation in the TMI-2 Accident, EGG-TMI-7402, November 1986.

4-11. Peterson, R. E., The Interaction Effect of Neighboring Holes or Cavities, With Particular Reference to Pressure Vessels and Rocket Cases, ASME Journal of Basic Engineering, Vol. 87, Series D, No. 4, December 1965.

4-12. Peterson, R. E., Stress Concentration Factors, John Wiley and Sons, New York, 1974, pp. 26.

4-13. Cook, R. D. and W. C. Young, 1985, Advanced Mechanics of Materials, Macmillan Publishing Company, pp. 183-193, 201-208.

4-14. R. Engelstad and Z. Feng, Mechanical and Structural Analysis of Corium Crusts, SMiRT 12, 1994.

4-15. M.L. Russell and R. K. McCardell, Three Mile Island Unit 2 Core Geometry, Nuclear Technology, Vol. 87, December 1989, pp. 865 - 874.

4-16. M. L. Russell, et al., TMI-2 Accident Evaluation Program Sample Acquisition and Examination Plan for FY-1988 and Beyond, EGG-TMI-7992, February 1988. 


\section{RECOMMENDATIONS FOR ASSESSMENT OF IMPROVED MODELS}

The assessment of the proposed new models can utilize a combination of detailed code-to-data comparisons, full plant calculations, TMI-2 analyses, and comparisons with more detailed models. The code-to-data comparisons can be used to assess the debris bed transitions and characteristics models, effective thermal conductivity models, and, to a more limited extent, molten pool natural circulation models. Full plant studies can be used to evaluate the overall integration of the models to ensure that "physically unrealistic' behavior and nodalization sensitivities have been significantly reduced over the current version of the code. These calculations can also be used to evaluate the coupled effects of debris formation and flow diversion. The TMI- 2 analyses can be used to assess the formation of debris beds, molten pool formation and growth, and molten pool crust failure when the debris beds and molten pool are covered by water. Comparisons with more mechanistic models can be used to assess the transition criteria, metallic and ceramic melt formation, debris heating and initial melting, and molten pool formation.

\subsection{Debris Transition and Characteristics}

The assessment of the transition between intact and debris bed geometries can utilize a large data base of bundle heating and melting experiments. The limited characterization using the experimental results discussed in Section 2.1 can be greatly expanded to cover the full range of conditions expected to be important in these models. For example, the effect of bundle size can be further assessed by looking at the metallographic results from the large CORA-7 and CORA-18 bundles, ${ }^{5-1}$ and full height FLHT-4 and FLHT-5 experiments. ${ }^{5-2,5-3}$ The effect of different initial heating rates can be assessed using data from ESBU-1 (relatively fast heating rates), ${ }^{5-4}$ SFD-ST (slow), ${ }^{5-5}$ and CORA-31 (slow). ${ }^{5-6}$ The ACCR-ST experiments ${ }^{5-7}$ can be used to assess the foaming of irradiated fuel at temperatures near $(\mathrm{U}, \mathrm{Zr}) \mathrm{O}_{2}$ melting. The ACRR MP-1 ${ }^{5-8}$ and MP-2 ${ }^{5-9}$ experiments can be used to further assess the transition to ceramic melting and blockage formation.

TMI-2 $2^{5-10}$ and other full plant calculations can also be used to assess the overall impact of these models. As discussed in Section 2.4, the existing models resulted in "physically unrealistic" behavior in some cases due to the coupling of thermal-hydraulics and debris bed formation processes.

\subsection{Debris Heating and Melting}

The assessment of the debris heating and melting models could be performed using a three stage process. First, as discussed in the Section 5.2.1, the effective thermal conductivity models for lower plenum loose debris could be assessed through comparison with the original data base for these models. Second, the detailed lower plenum debris models could be used to assess the more simplified in-core debris models to quantify the effect of detailed 2D heat conduction models and nodalization versus simplified lumped parameter models with a coarser nodalization. Third, the simplified initial melting and melt relocation models used for the incore and 2D lower plenum debris models could be assessed using the more detailed melt relocation models used in the DEBRIS code ${ }^{5-11}$. The DEBRIS models account for radial movement of melt within a rubble debris bed and thermal non-equilibrium between the melt and solid particles. However, it would be necessary to adjust DEBRIS effective thermal-conductivity models because a non-standard form of the correlations are used in the DEBRIS code (see Section 2.4.1). 


\subsubsection{Effective Thermal Conductivity Models for Lower Plenum Loose Debris}

Information discussed in Section 2 indicates that the Nasr, et al. correlation for predicting debris bed effective conductivity be incorporated into the SCDAP/RELAP5 code. This correlation has been shown to be consistent with data obtained from tests considering a range of particle diameters, materials, and temperatures. However, it is recommended that the Nasr, et al. correlation be assessed against a wider range of test conditions. For example, it is recommended that the assessment include data from tests considering multiple particle sizes, non-spherical particle geometries, a range of debris bed porosities, and a range of gas or vapor test pressures and types. Although additional test data may be included in this assessment, it is recommended that, as a minimum, the assessment include data from the references identified in Table 5-1. If this assessment indicates that the Nasr, et al. correlation more accurately predicts heat transfer in debris beds than the existing model, then the existing model should be replaced.

Table 5-1. Data sources for assessing porous debris bed effective conductivity model.

\begin{tabular}{|c|c|c|}
\hline Phenomena & Source & $\begin{array}{c}\text { Reference } \\
\text { Number }\end{array}$ \\
\hline \multirow[t]{6}{*}{ Particle composition } & Imura and Takeoshi & $5-11$ \\
\hline & Nasr, Viskanta, and Ramadhyani & $5-12$ \\
\hline & Yagi and Kunii & $5-13$ \\
\hline & Schotte & $5-14$ \\
\hline & Swift & $5-15$ \\
\hline & Donne and Sordon & $5-17$ \\
\hline Multiple particle compositions & Donne and Sordon & $5-17$ \\
\hline \multirow[t]{2}{*}{ Particle temperature } & Nasr, Viskanta, and Ramadhyani & $5-12$ \\
\hline & Bauer and Schlünder & $5-16$ \\
\hline \multirow[t]{5}{*}{ Particle size and shape } & Imura and Takeoshi & $5-11$ \\
\hline & Nasr, Viskanta, and Ramadhyani & $5-12$ \\
\hline & Yagi and Kunii & $5-13$ \\
\hline & Bauer and Schlünder & $5-16$ \\
\hline & Donne and Sordon & $5-17$ \\
\hline \multirow[t]{3}{*}{ Presence of multiple particle sizes } & Imura and Takeoshi & $5-11$ \\
\hline & Bauer and Schlünder & $5-16$ \\
\hline & Donne and Sordon & $5-17$ \\
\hline
\end{tabular}


Table 5-1. Data sources for assessing porous debris bed effective conductivity model. (Continued)

\begin{tabular}{|l|l|c|}
\hline \multicolumn{1}{|c|}{ Phenomena } & \multicolumn{1}{|c|}{ Source } & \multicolumn{1}{c|}{$\begin{array}{c}\text { Reference } \\
\text { Number }\end{array}$} \\
\hline Debris bed porosity & Imura and Takeoshi & $5-11$ \\
\cline { 2 - 3 } & Nasr, Viskanta, and Ramadhyani & $5-12$ \\
\cline { 2 - 3 } & Botterill, Salway, and Teoman & $5-18$ \\
\hline Influence of particle oxide layer & Imura and Takeoshi & $5-11$ \\
\cline { 2 - 3 } & Donne and Sordon & $5-17$ \\
\cline { 2 - 3 } & Zehner & $5-19$ \\
\hline Influence of vapor or gas pressure & Imura and Takeoshi & $5-11$ \\
\cline { 2 - 3 } & Yagi and Kunii & $5-13$ \\
\cline { 2 - 3 } & Schotte & $5-14$ \\
\hline
\end{tabular}

\subsection{Cohesive Blockage and Molten Pool Formation and Growth}

The assessment of cohesive blockage and molten pool formation and growth can be performed using a combination of TMI-2 and other full plant calculations and comparisons with more detailed models. The first stage of the assessment would be to assess the changes in volume and internal porosity of metallic and cohesive blockage regions during initial formation and in some cases remelting. This stage could utilize bubble growth and formation models developed for fission product release in metallic and ceramic fuels, and core-concrete interactions as discussed in Section 3.4.3 to establish the influence of pressure and timeat-temperature. The second stage could utilize a detailed fluid dynamics code, such as FIDAP, to assess molten pool behavior. Specific recommendations for these calculations are provided in Section 5.3.1. The third stage would be to assess the behavior using data from TMI-2, comparing the timing and position of the predicted blockages and molten pool at different phases of the accident. The fourth stage would be to assess the overall behavior of the models using a variety of full plant calculations.

\subsubsection{Molten Pool Natural Convection}

Although analyses discussed in Section 3 indicate that there are sufficient data to modify the current SCDAP/RELAP5 molten pool models, it should be emphasized that there is still considerable uncertainty associated with many of the proposed model modifications. Furthermore, as discussed in Section 3.2.3, there are no integral test data available for assessing the revised model. Hence, it is recommended that the revised model be assessed against predictions from a more detailed computational fluids dynamic code, FIDAP, ${ }^{5-20}$ that is capable of predicting transient and steady-state natural convection phenomena and vapor transport in the molten pool. Specific steps for this assessment are discussed below.

Because both FIDAP and SCDAP/RELAP5 models consider crust formation and melting, these assessment calculations will also provide some insights about the impact of melting and solidification phenomena on molten pool heat transfer. Finally, it is suggested that multiple component pools be 
considered in these calculations to assess differences between predictions from the revised COUPLE model and the more detailed, computational fluids dynamics code, FIDAP.

It is suggested that the revised model be assessed by comparing results from revised COUPLE model in SCDAP/RELAP5 with FIDAP from the following three calculations:

- Steady-state natural convection problem. Heat generation rates, melt material composition, and pool sizes will be varied for the range of values listed in Table 5-1. Semicircular, torispherical, and hemispherical geometries will be considered. Directional heat fluxes will be compared to assess the model's validity.

- Transient natural convection problem. The analyses considered for the steady-state natural convection problem will be repeated to investigate transient pool behavior. Time-dependent directional heat fluxes will be compared to assess the model's validity.

- Vapor transport problem. The analyses considered for the steady-state natural convection problem will be repeated to investigate vapor transport. In these calculations, pool void fractions will be varied from 0 to 0.4 . Directional heat fluxes will be compared to assess the model's validity.

\subsection{Molten Pool Crust Failure}

The proposed models use a bounding approach to predict crust failure. The first assessment should determine whether known experimental or actual accident data fall within the bounding solutions. As indicated in Section 4, data to verify molten pool crust failure is very limited, with the TMI-2 accident providing the only full-scale, integral data. A SCDAP/RELAP5 simulation of the TMI-2 accident should be run using the bounding cases describes by Tables 4-1 and 4-2 to verify that the timing of actual crust failure falls within the bounding solutions. The models would then be assessed using comparisons with more detailed coupled thermal and structural models using idealized geometries to verify the simplified structural models described in Section 4 adequately represent the structural response of the crust over a wide range of conditions. As noted in the introduction to this section, the model-to-model assessment will not be capable of identifying systematic biases in the results associated with uncertainties in crust mechanical properties or no homogeneities in the crust. However sensitivity studies could be performed to quantify the influence of these uncertainties.

\subsection{Remaining Uncertainties}

If the recommendations provided in the preceding sections are followed, it should be possible to provide reasonable quantitative uncertainties for individual models with the possible exception of the following models.

(1) Fuel desintering - fuel desintering occurred in a very limited set of experiments, yet the potential impact could be relatively large if a large fraction of the fuel in the core shatters into a fine powder. Without a detailed review of the specific conditions leading to fuel desintering to insure that the basic mechanisms are understood, the correlational approach suggested at the preferred options may have relatively large uncertainties. 
(2) Void formation in molten pools - Voids were observed in all of the metallographic cross-sections of previously molten ceramic masses. In addition, metallic masses with control rod or structural material present showed a foamy appearance. In addition to the obvious impact on blockage formation, the appearance of voids in molten pools can have a significant impact on the natural circulation heat transfer to the surrounding crust. As a consequence, the selection and assessment of an appropriate void fraction model or correlation may have a dominant impact on the overall uncertainties in predicted molten pool behavior.

(3) Molten pool crust failure - The development of a defensible structural failure model for crust failure may be one of the most critical model improvement activities since the timing and location of molten pool crust failure will have a dramatic impact on the melt masses relocating into the lower plenum. In the most extreme cases, the crust failure model may result in a gradual draining of melt into the lower plenum if the top or upper portion of the side crust fails. By contrast the existing model assumes a very massive slumping by draining the entire molten pool. However, the TMI-2 accident provides the only data which can be used to assess this model. As a consequence, the assessment of the model using more detailed thermal and structural failure models will be critical in quantifying the uncertainties in the model.

One important aspect of the assessment of crust failure and other models using comparisons with other more detailed models must be noted. Unlike code-to-data comparisons, using experiments with prototypical materials, both the proposed models and more detailed models will use the same materials properties database. Thus, model-to-model variations reflect a lower bound on uncertainties on the models. By contrast, code-to-data comparisons tend to product conservative estimates because any variation between calculated and measured responses include modeling, experiment, and material property uncertainties. The assessment of uncertainties in the models using simulant tests falls in between these two extremes because the uncertainties in material properties are not included but other experimental uncertainties are.

\subsection{References}

5-1. S. Hagen, et al., CORA Experiments on the Materials Behavior of LWR Fuel Rod Bundles at High Temperatures, NUREG/CP-0119, Proceedings of the 19th Water Reactor Safety Meeting, April 1992.

5-2. D. Lanning, et al., Data Report - Full-Length High-Temperature Experiment 4, PNL-6368, January 1988.

5-3. D. Lanning, et. al., Data Report - Full-Length High-Temperature Experiment 5, PNL-6540, April 1988.

5-4. S. Hagen and S. O. Peck, "Temperature Escalation of Zircaloy-Clad Fuel Rods and Bundles under .Severe Fuel Damage Conditions", International Meeting on LWR Severe Accident Evaluation, Cambridge, August 28 - September 1, 1983.

5-5. A. D. Knipe, et al., PBF Severe Fuel Damage Scoping Test - Test Results Report, NUREG/CR4683, EGG-2413, August 1986

5-6. S. Hagen, et. al., BWR Slow Heatup Test CORA-31: Test Results, KfK 5383, to be published.

5-7. M. Allen, et. al., Fission Product Release and Fuel Behavior of Irradiated Light Water Reactor Fuel Under Severe Accident Conditions - the ACRR ST-1 Experiment, NUREG/CR-5345, SAND89-0308, November 1991. 
5-8. R. D. Gasser, et al., Late Phase Melt Progressin Experiment - MP-1 Results and Analyses, NUREG/CR-5874, SAND92-0804.

5-9. $\quad$ R. Gauntt, "The Late Phase Melt Progression Experiments, Results and PIE," Late Phase Melt Progression Peer Review Meeting, Rockville, January 1994.

5-10. J. Broughton, et al., "A Scenario of the Three Mile Island Unit 2 Accident," Nuclear Technology, 87, August 1989, pp. 34-53.

5-11. S. Imura and E. Takegoshi, "Effect of Gas Pressure on the Effective Thermal Conductivity of Packed Beds," Heat Transfer Japanese Research, Vol. 3, No. 4, p. 13, 1974.

5-12. K. Nasr, R. Viskanta, and S. Ramadhyani, "An Experimental Evaluation of the Effective Thermal Conductivities of Packed Beds at High Temperatures," to be published in the ASME Journal of Heat Transfer.

5-13. S. Yagi and D. Kunii, A.I.Ch.E. J., Vol. 3. p. 373, 1957.

5-14. W. Schotte, A.I.Ch.E.J. Vol. 6, p. 63, 1960.

5-15. D. L. Swift, Int. J. Heat Mass Transfer, 9, p. 1061, 1966.

5-16. R. Bauer and E. U. Schlünder, "Effective Radial Thermal Conductivity of Packing in Gas Flow. Part II. Thermal Conductivity of the Packing Fraction without Gas Flow," International Chemical Engineering, Vol. 18, No. 2, pp. 189-204.

5-17. M. D. Donne and G. Sordon, "Heat Transfer in Pebble Beds for Fusion Blankets," Fusion Technology, Vol. 17, July 1990, pp. 597-635.

5-18. J. S. M. Botterill, A. G. Salvaw, and Y. Teoman, "The effective thermal conductivity of high temperature particulate beds - I. Experimental determination," Int. J. Heat Mass Transfer, Vol. 32, No. 3, pp. 385-393, 1989.

5-19. P. Zehner, Dissertation, University of Karlsruhe (TH), 1972.

5-20. Fluid Dynamics International, Inc. FIDAP (Fluids Dynamics Analysis Package) 7.0 Users Manual, 1993. 


\section{APPENDIX A}

A-1 


$$
\text { A-2 }
$$




\section{REVIEW OF MOLTEN POOL NATURAL CIRCULATION HEAT TRANSFER EXPERIMENTS AND CORRELATIONS}

During a severe accident, there is the potential for molten pools to form that are internally heated by fission product decay heat. Two locations within the reactor vessel have been identified where such pools may form - the core region when molten fuel material may relocate to form a molten pool surrounded by a ceramic crust and the lower plenum of the reactor vessel when fuel material may relocate from the core region to form a molten pool surrounded by a ceramic crust. Because it is important to model heat transfer from molten pools during a severe accident, an assessment of the current model in SCDAP/RELAP5 was performed. Major tasks of this assessment included reviewing previous work related to modeling heat transfer from a molten pool, assessing the current SCDAP/RELAP5 models for predicting molten pool heat transfer, and making recommendations for improving SCDAP/RELAP5 models for predicting heat transfer from a molten pool.

Key questions addressed within this assessment are discussed below:

- What are key phenomena impacting heat transfer from a molten pool during a severe accident? Within this study, the effects of parameters, such as test fiuid material properties, test facility geometry, and the range of key scaling parameters, were reviewed to assess differences in predictions for the range of parameters of interest to severe accident analysis. In addition, the impact of phenomena, such as transient pool behavior, prototypic material property behavior, vapor transport within a pool (due to vaporization of lower-melting point materials and fission gas release), melting and solidification within the pool, and multiple fiuids or layers within a pool, were reviewed.

- What data are available for predicting heat transfer from a molten pool? As indicated within this appendix, most data for predicting heat transfer from a molten pool were obtained from experiments investigating steady-state heat transfer. A limited amount of analytical and experimental studies have also been performed to investigate transient heat transfer from molten pools. Even less data were found that were applicable to predicting the effects of other phenomena impacting heat transfer from a molten pool, such as prototypic material behavior, vapor transport, etc.

- $\quad$ How can the existing SCDAP/RELAP5 models be improved to more accurately predict available data? The uncertainty associated with the data is reported where possible. In addition, scoping calculations are performed to assess the possible impact of phenomena on heat transfer from a molten pool

- Is the model currently within SCDAP/RELAP5 adequate for predicting available data? Scoping calculations are performed to assess differences between various steady-state correlations and differences that various phenomena will have on molten pool heat transfer predictions in a representative SCDAP/RELAP5 calculation.

Section A1 of this appendix provides background information for modeling natural convection heat transfer and representative values of parameters used to characterize natural convection heat transfer from 
molten pools that may occur in a reactor vessel during a severe accident. A summary description of the current model in SCDAP/RELAP5/MOD3.1 for predicting heat transfer from a molten pool is also included in Section A1. Section A2 summarizes previous experimental and numerical analyses that have been performed to characterize steady-state natural convection heat transfer. Previous work to investigate transient heat transfer from a molten pool is summarized in Section A3. Previous experiments investigating molten pool heat transfer with prototypic materials are summarized in Section A4. Section A5 summarizes previous work to investigate the effects of vapor in a molten pool on heat transfer. The impact of solidification and melting on natural convection is discussed in Section A6, and the effects of multiple layers in a molten pool are discussed in Section A7. Section A8 summarizes conclusions and recommendations from this study for improving the current model in SCDAP/RELAP5.

\section{A1. BACKGROUND}

In this section, dimensionless groups used to characterize natural convection heat transfer from a molten pool are reviewed. Values for these dimensionless groups are quantified for representative severe accident conditions. In addition, this section includes a brief description of the current model used in SCDAP/RELAP5/MOD3.1 for predicting heat transfer from a molten pool.

\section{A1.1 Characteristic Dimensionless Groups}

For steady-state heat transfer, data are usually correlated in terms of the surface-averaged Nusselt number, $\mathrm{Nu}$, a modified Rayleigh number, $\mathrm{Ra}$, and the fluid Prandtl number, $\mathrm{Pr}$, which are defined by

$$
\begin{aligned}
& \mathrm{Nu}=\frac{\mathrm{hL}}{\mathrm{k}}=\frac{\mathrm{q}^{\prime \prime}}{\frac{\mathrm{k}}{\mathrm{L}} \Delta \mathrm{T}} \\
& \mathrm{Ra}=\frac{\mathrm{g} \beta \mathrm{q}^{\prime \prime} \mathrm{L}^{5}}{\alpha v \mathrm{k}} \\
& \operatorname{Pr}=\frac{\mathrm{v}}{\alpha}
\end{aligned}
$$

where

$$
\begin{array}{ll}
\mathrm{k} & =\quad \begin{array}{l}
\text { thermal conductivity }(\mathrm{W} / \mathrm{m} \bullet \mathrm{K}) \\
\mathrm{h}
\end{array} \quad \begin{array}{l}
\text { average heat transfer coefficient }\left(\mathrm{W} / \mathrm{m}^{2} \bullet \mathrm{K}\right) \\
\mathrm{L}
\end{array} \quad \begin{array}{l}
\text { a characteristic length; in a hemispherical geometry, this study has used the } \\
\text { radius; and in a rectangular geometry, this study has used the pool height }(\mathrm{m})
\end{array} \\
\mathrm{g} & =\quad \text { acceleration due to gravity }\left(\mathrm{m} / \mathrm{s}^{2}\right)
\end{array}
$$




$\begin{array}{lll}\beta & = & \text { volumetric coefficient of thermal expansion }\left(\mathrm{K} / \mathrm{m}^{3}\right) \\ \alpha & = & \text { thermal diffusivity of the fluid in the pool }\left(\mathrm{m}^{2} / \mathrm{s}\right) \\ v & = & \text { kinematic viscosity of the fluid in the pool }\left(\mathrm{m}^{2} / \mathrm{s}\right) \\ \mathrm{q}^{\prime \prime \prime} & = & \text { volumetric heat generation rate within the pool }\left(\mathrm{W} / \mathrm{m}^{3}\right) \\ \mathrm{q}^{\prime \prime} & = & \text { heat flux from the pool }\left(\mathrm{W} / \mathrm{m}^{2}\right) \\ \Delta \mathrm{T} & = & \text { difference between pool peak temperature and pool melting temperature }(\mathrm{K}) .\end{array}$

It should be noted that various references differ in their definition of the Rayleigh number. In this study, consistent definitions for the Rayleigh number are used for each geometry. Therefore, correlations reviewed in Section A2 were modified to reflect the consistent Rayleigh number selected for each geometry. In each section, the characteristic length used to calculate the Rayleigh and Nusselt numbers is identified.

For transient heat transfer, data may be correlated using the Fourier number, Fo, and the transient Rayleigh number, $\mathrm{Ra}^{\prime}$, which are defined by

$F_{0}=\frac{\alpha t}{L^{2}}$
$\mathrm{Ra}^{\prime}=\frac{\mathrm{Ra}}{\mathrm{Nu}}$

For a rectangular geometry, the transient Rayleigh number may be reduced to

$\mathrm{Ra}^{\prime}=\frac{\mathrm{g} \beta \Delta \mathrm{TL}^{3}}{\alpha \nu}$

For other geometries, evaluation of Equation (A-5) requires consideration of the volume to area ratio (see Section A3).

\section{A1.2 Typical Ranges for Molten Pool Heat Transfer Parameters}

As part of this task, the applicability of previous experimental work is assessed by comparing the range of conditions over which the data were obtained to the range of conditions expected during a severe LWR accident. Therefore, representative ranges for the dimensionless groups defined in Section A1.1 were quantified using representative upper and lower input values and using results from a representative SCDAP/RELAP5/MOD3.1 calculation. Values estimated from these sources are summarized in Table A-1. 
Table A-1. Ranges for variables used to estimate pool heat transfer parameters.

\begin{tabular}{|c|c|c|c|}
\hline Parameter/Units & \multicolumn{3}{|c|}{ Condition } \\
\hline Thermal Properties & $\begin{array}{c}\text { Ceramic } \\
\text { Material } \\
\left(\mathbf{U O}_{\mathbf{2}} \text { at } \sim \mathbf{3 2 0 0} \mathbf{K}\right)\end{array}$ & $\begin{array}{c}\text { Metallic Material } \\
\text { (SS-304 at } \sim 1700 \mathrm{~K})\end{array}$ & $\begin{array}{c}80 \% \mathrm{UO}_{2} / 20 \% \\
\mathrm{ZrO}_{2} \text { (by weight) } \\
\text { Compound } \\
\text { (at } \sim 2900 \mathrm{~K} \text { ) }\end{array}$ \\
\hline$\rho, \mathrm{kg} / \mathrm{m}^{3}$ & 8400 & 7250 & 9300 \\
\hline$c_{\mathrm{p}}, \mathrm{J} / \mathrm{kgK}$ & 500 & 560 & 650 \\
\hline $\mathrm{k}, \mathrm{W} / \mathrm{m} \cdot \mathrm{K}$ & 3.8 & 38 & 3.8 \\
\hline$\beta, \mathrm{K}^{-1}$ & $1.6 \mathrm{e}-4$ & $3.5 e-4$ & $7.1 e-5$ \\
\hline$\alpha, \mathrm{m}^{2} / \mathrm{s}$ & $9.0 \mathrm{e}-7$ & $9.4 \mathrm{e}-6$ & $6.2 \mathrm{e}-7$ \\
\hline $\mathrm{v}, \mathrm{m}^{2} / \mathrm{s}$ & $5.4 e-7$ & $3.2 \mathrm{e}-7$ & $4.8 \mathrm{e}-7$ \\
\hline $\operatorname{Pr}$ & 0.6 & 0.03 & 0.8 \\
\hline $\begin{array}{l}\text { Heat Source and } \\
\text { Geometry }\end{array}$ & \multicolumn{2}{|c|}{ Lower Bound/Upper Bound } & Reference A-3 \\
\hline$q^{\prime \prime \prime}, W / m^{3}$ & \multirow{2}{*}{\multicolumn{2}{|c|}{$\begin{array}{c}1.0 \mathrm{e} 5 / 2.0 \mathrm{e} 6 \\
1.0 / 2.4\end{array}$}} & $1.98 \mathrm{e} 6$ \\
\hline $\mathrm{L}, \mathrm{m}$ (core) & & & Figure A-1 \\
\hline $\mathrm{L}, \mathrm{m}$ (vessel) & \multicolumn{2}{|c|}{$1.0 / 2.8$} & Not available \\
\hline
\end{tabular}

Upper and lower ranges listed in Table A-1 were quantified using information in Reference A-1 and Reference A-2. These values were obtained by considering a range of debris conditions and reactor designs. Metallic and debris compositions were considered in estimating values for debris thermal properties. Volumetric heat generation rates were quantified using results from previous calculations. Pool characteristic lengths were quantified considering pool sizes possible in the core and in the vessel lower plenum (i.e., upper bounds for the pool radius were based on core and vessel radii).

In addition, values for these dimensionless groups were quantified based on conditions predicted in a recently completed set of SCDAP/RELAP5/MOD3.1 calculations performed for a Surry station blackout event. ${ }^{A-3}$ In these calculations, a molten pool is predicted to form in the core region at approximately 4.9 hours after reactor scram. At approximately 5.4 hours, the molten material within the pool in the core region (approximately $49,000 \mathrm{~kg}$ of $\mathrm{UO}_{2}$ and $12,000 \mathrm{~kg}$ of $\mathrm{ZrO}_{2}$ ) is predicted to relocate to the lower head, where it forms a second molten pool. Figure A-1, Figure A-2, and Figure A-3 illustrate the time-dependent effective radius of the pool in the core region, the peak temperature of the material in the core region, and the RCS pressure in the core region. 


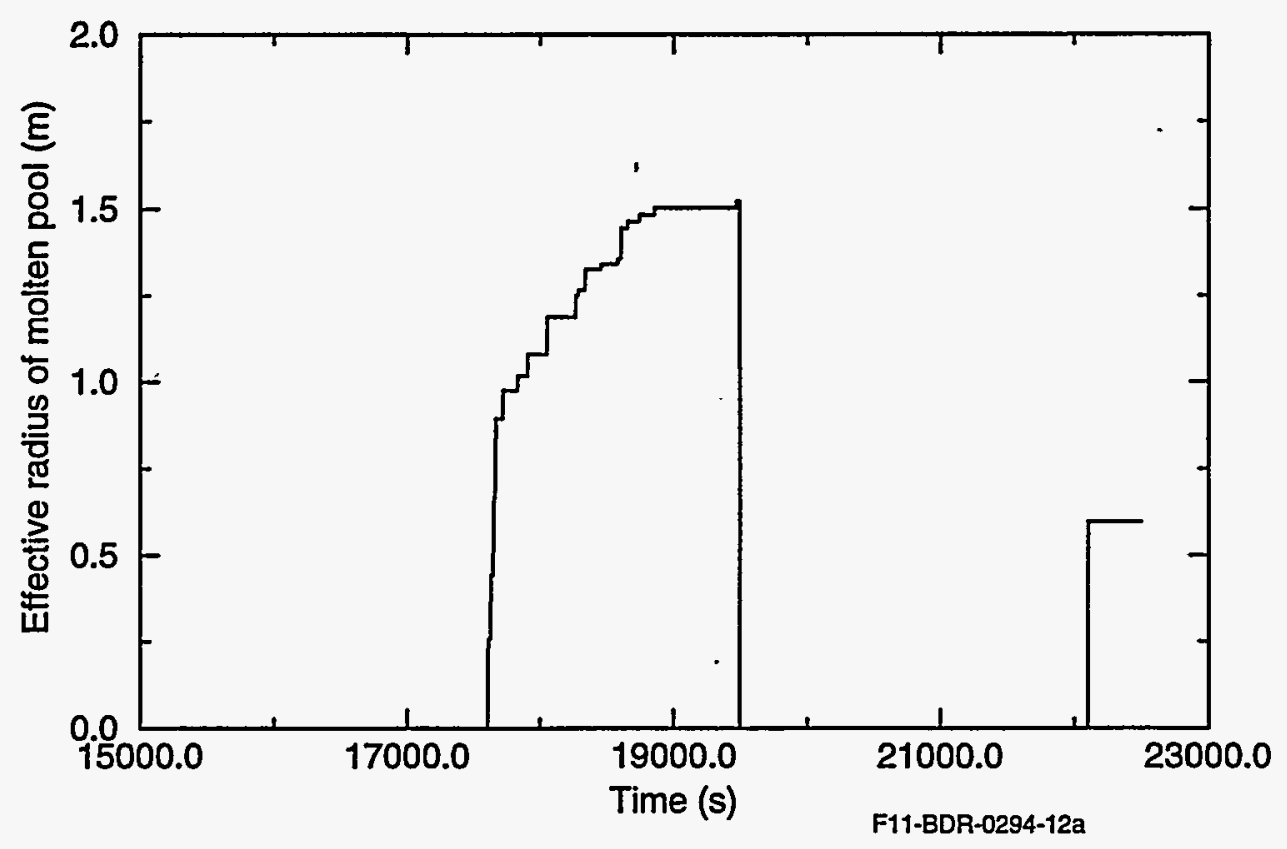

Figure A-1. Time-dependent effective radius of molten pool in the core region.

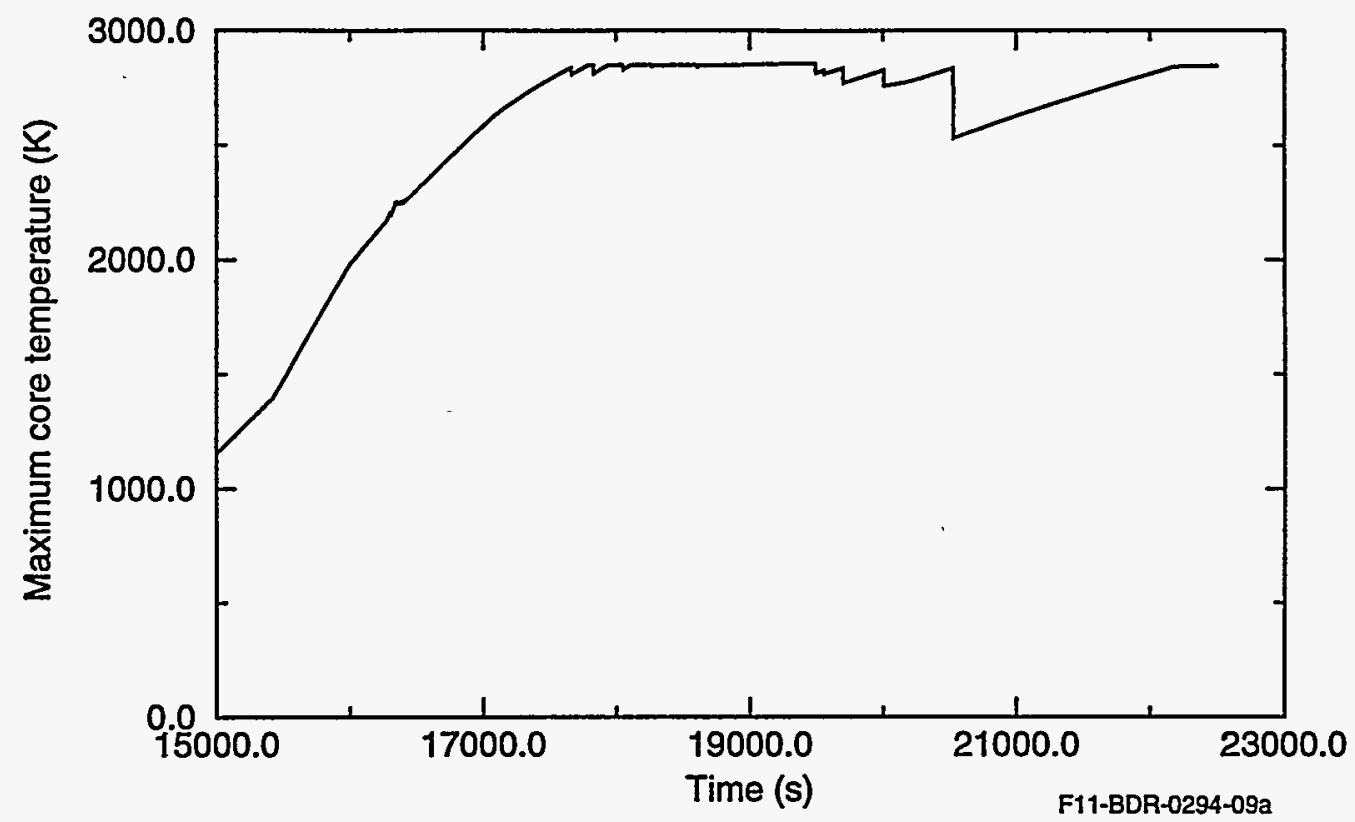

Figure A-2. Peak temperature of core region. (Note that molten pool is not formed until after $17800 \mathrm{sec}-$ onds). 


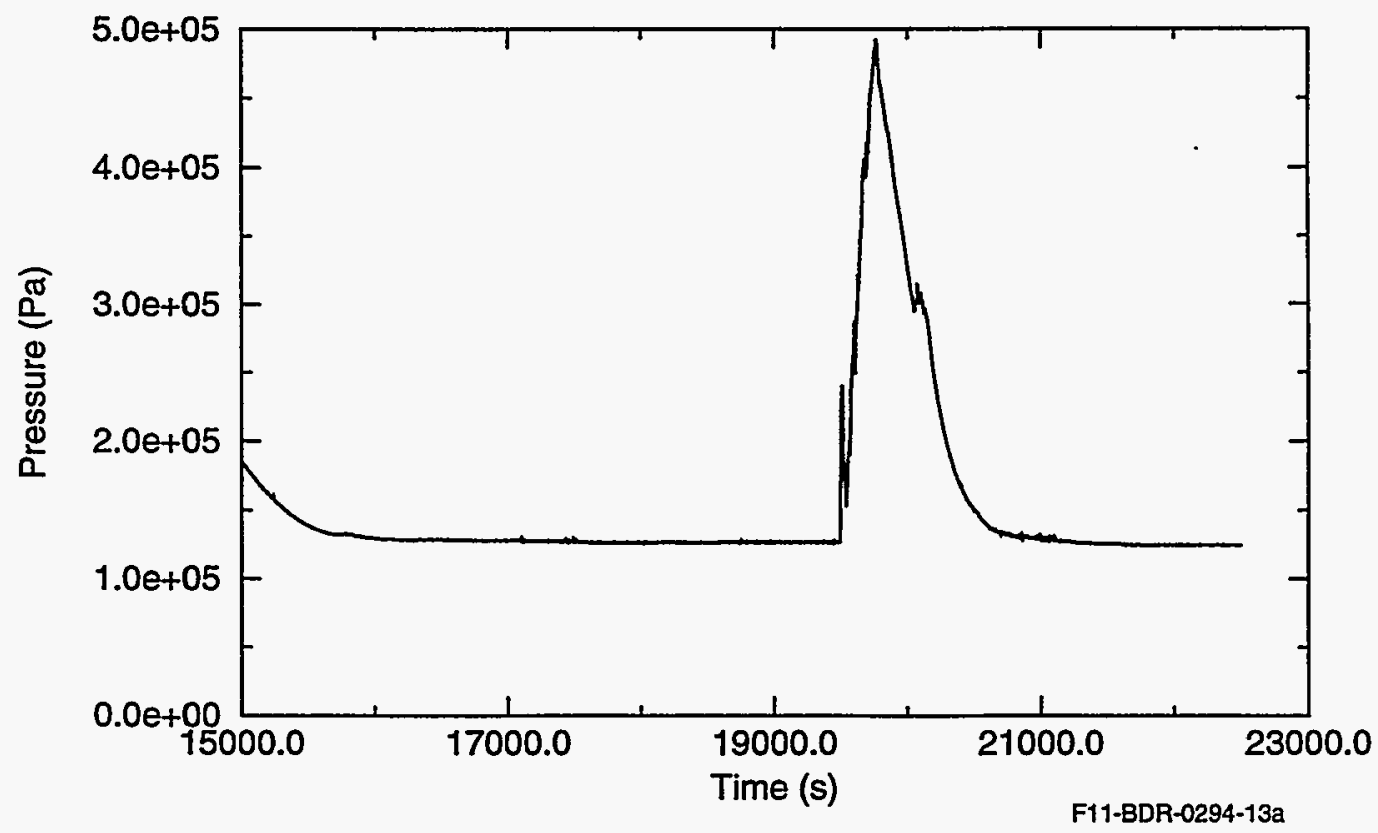

Figure A-3. Steam pressure at top of molten pool in the core region.

Using the values in Table A-1, the Rayleigh number applicable to pools in the core region is estimated to range from $10^{12}$ to $10^{17}$. For pools that form in the lower head of the vessel, the Rayleigh number is estimated to range from $10^{13}$ to $10^{17}$. Debris Prandtl numbers are estimated to range from 0.03 to 0.8 . As will be discussed in Section A2, this range of parameters is considerably different than the range for which most natural circulation heat transfer data for fluids with internal heating were obtained.

\section{A1.3 SCDAP/RELAP5/MOD3.1 Molten Pool Heat Transfer Model}

For reference purposes, a brief description of the method used to estimate heat transfer from a molten pool in SCDAP/RELAP5 is summarized below. Note that SCDAP/RELAP5 can model the formation of a molten pool in two locations: the core region and the vessel lower head. In the core region, various axial and radial locations are divided up into nodes; whereas in the vessel lower head, a two-dimensional finite element conduction model, based on the COUPLE code, is used to model the molten pool and vessel. As discussed below, the method used in SCDAP/RELAP5 to estimate heat transfer to the pool boundaries is similar for pools forming in either location. Potential uncertainties associated with the current method used to predict heat transfer are also identified in this section.

In order to estimate heat transfer from a molten pool, SCDAP/RELAP5 maps the volume of molten material onto a pool with hemispherical geometry. Natural convection heat transfer coefficients from a hemispherical pool are estimated using the following steady-state correlations recommended by Mayinger, Jahn, Reineke, and Steinbrenner. ${ }^{\mathrm{A}-9, \mathrm{~A}-10}$ Descriptions of the experimental tests used to obtain these correlations can be found in Section A2.2.1. 
$\mathrm{Nu}_{\mathrm{u}}=0.36 \mathrm{Ra}^{0.23}$

$\mathrm{Nu}_{\mathrm{d}}=0.54 \mathrm{Ra}^{0.18}$.

As discussed in Section A2.2.1, these correlations are based on experimental data obtained from a "slice" hemispherical geometry. In the experiments used to obtain these correlations, the Rayleigh number for the experimental fluid was varied from $1 \times 10^{7}$ to $5 \times 10^{10}$, and the Prandtl number for the experimental fluid, water, was around 7 . Hence, the ranges for which these correlations were obtained are considerably different than the ranges expected for severe accident conditions.

According to data presented in Reference A-10, the heat transfer along the bottom surface varies with location. A maximum downward heat flux, approximately twice the average downward heat flux, occurs at the upper edge of the pool; and the minimum heat flux occurs at the bottom center of the pool. In SCDAP/ RELAP5/MOD3.1, the total volume of molten material, irrespective of material composition, is mapped onto a hemispherical shape and heat transfer coefficients estimated for this idealized, hemispherical, geometry are mapped back into the actual configuration of the molten pool. For example, the heat transfer coefficient at the bottom center of the idealized pool is applied to the bottom center of the actual pool.

Note that two of the above simplifying assumptions may lead to erroneous code predictions. First, the assumption that the entire volume of molten material, irrespective of melt composition, can be used to estimate an effective radius for the pool neglects the effects of density-stratified, multiple layers on natural convection. As will be discussed in Section A7, data indicate that models for predicting natural convection in multiple component pools should consider phenomena, such as if there is internal heating present in one or both layers within the pool, the potential for crusts to form at the interface between the layers, and if material interactions will occur between the melt layers. Second, the assumption that the molten volume of material within the pool can be mapped onto a hemispherical geometry may lead to erroneous predictions for heat transfer coefficients. Scoping calculations performed in Section A2.3 illustrate the errors that may be introduced when pool geometry effects are ignored.

\section{A2. STEADY-STATE INVESTIGATIONS}

A representative sample of experimental and numerical investigations performed to study heat transfer from molten pools with natural convection and volumetric heating are summarized in this section. In addition to the original references reporting these investigations, the present study also considered information in previous reviews of natural convection (e.g., Reference A-4 through Reference A-8).

In general, most studies were performed using Joule heating of an electrically conducting fluid with alternating current. The fluid is typically an aqueous solution with a Prandtl number of around 7 , and Rayleigh numbers investigated were rarely above $10^{12}$. Several geometries have been studied. Section A2.1 summarizes studies for flat (rectangular) geometries, and Section A2.2 summarizes studies for curved (spherical, hemispherical, cylindrical, and torispherical) geometries. General comments about the existing data base and recommendations for modeling pools during a severe accident are summarized in Section A2.3. 


\section{A2.1 Flat Geometries}

Considerable experimental and numerical studies have been performed to investigate heat transfer from molten pools with volumetric heat sources in rectangular layers and cavities. Representative studies performed for these geometries are discussed in this section. Table A-2 summarizes the studies reviewed in this section. Correlations developed for predicting heat transfer are graphically compared in Figure A-7 through Figure A-9. Figure A-7 compares correlations developed for rectangular cavities and layers using experimental data. Figure A-8 compares numerically and experimentally-developed correlations for rectangular layers and cavities with adiabatic side boundaries and constant temperature upper and lower boundaries. Figure A-9 compares numerically and experimentally-developed correlations for rectangular layers and cavities with adiabatic lower and side boundaries and constant temperatures upper boundaries. In these figures, all of the correlations were evaluated for a fluid with a Prandtl number of 7 . Although some numerical studies considered fluids with lower Prandtl numbers, most of the data were obtained for fluids with a Prandtl number of 7 .

Curves in Figure A-7 through Figure A-9 are representative of results from previous studies for several reasons. Although there are limited data for Rayleigh numbers between $10^{12}$ and $10^{14}$, most investigations were conducted for fluids with Rayleigh numbers less than $10^{12}$. Most data suggest that the Nusselt number is proportional to the Rayleigh number to approximately the $1 / 5$ th power, thus, suggesting that the heat transfer coefficient is nearly independent of the test facility's length. The data are fairly consistent in illustrating that heat transfer is significantly higher toward the upper and side surfaces than towards the lower surface. There is little scatter among values predicted by correlations developed from experiments using geometries with similar boundary conditions. As indicated in Figure A-7, data may vary by less than $10 \%$, which is comparable to the 5 to $15 \%$ uncertainty estimated by many of the experimentalists for their results. Based on the work reviewed in this section, it is recommended that the correlations proposed by Steinberner and Reineke are the most applicable for pools that may form during a severe accident. These correlations were obtained from facilities at higher Rayleigh numbers (between $8 \mathrm{x}$ $10^{12}$ and $4 \times 10^{13}$ ), and they appear to be consistent with much of the data obtained from lower Rayleigh number investigations.

Additional recommendations based on the data reported in this section and in Section A2.2 may be found in Section A2.3. Graphical comparisons of correlations presented in these two sections are also found in Section A2.3. 
Table A-2. Summary of experimental and numerical natural convection studies reviewed for rectangular cavities.

\begin{tabular}{|c|c|c|c|c|c|}
\hline Geometry & Correlation & $\mathrm{Ra}^{\mathrm{a}}$ & $\operatorname{Pr}$ & $\mathrm{L} / \mathrm{D}$ & $\begin{array}{l}\text { Reference, } \\
\text { Type of Data }\end{array}$ \\
\hline \multirow{4}{*}{$\underbrace{y_{2}^{T \text { oonstant }}}_{D}$} & $\begin{aligned} \mathrm{Nu}_{\mathrm{u}} & =0.345 \mathrm{Ra}^{0.223} \\
\mathrm{Nu}_{\mathrm{d}} & =1.389 \mathrm{Ra}^{0.095}\end{aligned}$ & $4 \times 10^{4}$ to $5 \times 10^{5}$ & 7 & $<0.5$ & $\begin{array}{c}\text { Mayinger, Jahn, Reineke, } \\
\text { and Steinbrenner }[A-9, \text { A-10] } \\
\text { Experimental }\end{array}$ \\
\hline & $\begin{array}{c}\mathrm{Nu}_{\mathrm{u}}=0.292 \mathrm{Ra}^{0.23} \operatorname{Pr}^{0.085} \\
\mathrm{Nu}_{d}=1.235 \mathrm{Ra}^{0.1}\end{array}$ & $10^{5}$ to $2 \times 10^{10}$ & 0.04 to 5 & $<0.5$ & $\begin{array}{c}\text { Mayinger, Jahn, Reineke, } \\
\text { and Steinbrenner }[A-9, A-10] \\
\text { Numerical }\end{array}$ \\
\hline & $\begin{array}{l}N u_{u}=0.345 \mathrm{Ra}^{0.233} \\
N u_{d}=1.389 \mathrm{Ra}^{0.095}\end{array}$ & $\begin{array}{c}1 \times 10^{13} \text { to } 4 \times 10^{13} \\
4 \times 10^{13}\end{array}$ & 7 & 1 & $\begin{array}{c}\text { Steinberner and Reineke } \\
{[A-11]} \\
\text { Experimental }\end{array}$ \\
\hline & $\begin{array}{l}\mathrm{Nu}_{\mathrm{u}}=0.329 \mathrm{Ra}^{0.236} \\
\mathrm{Nu}_{\mathrm{d}}=1.43 \mathrm{Ra}^{0.094}\end{array}$ & $4 \times 10^{4}$ to $2 \times 10^{7}$ & 5.8 to 6.4 & 0.05 to 0.25 & $\begin{array}{l}\text { Kulacki and Goldstein [A-12] } \\
\text { Experimental }\end{array}$ \\
\hline & $\begin{array}{c}\mathrm{Nu}_{\mathrm{u}}=0.368 \mathrm{Ra}^{0.23} \mathrm{Pr}^{0.085} \\
\mathrm{Nu}_{\mathrm{d}}=1.235 \mathrm{Ra}^{0.1}\end{array}$ & $10^{5}$ to $2 \times 10^{10}$ & 0.04 to 5 & $<0.5$ & $\begin{array}{c}\text { Mayinger, Jahn, Reineke, } \\
\text { and Steinbrenner }[A-9, \text { A-10] } \\
\text { Numerical }\end{array}$ \\
\hline$g_{L}$ & $\begin{aligned} \mathrm{Nu}_{\mathrm{u}} & =0.328 \mathrm{Ra}^{0.23} \\
N u_{d} & =1.041 \mathrm{Ra}^{0.119}\end{aligned}$ & $2 \times 10^{5}$ to $2 \times 10^{8}$ & 6.5 & $\ll 1$ & $\begin{array}{c}\text { Emara and Kulacki [A-14] } \\
\text { Numerical }\end{array}$ \\
\hline$C^{T=\text { conssint }}$ & $\mathrm{Nu}_{\mathrm{u}}=0.338 \mathrm{Ra}^{0.227}$ & $4 \times 10^{3}$ to $4 \times 10^{12}$ & 2.8 to 6.9 & 0.025 to 0.5 & $\begin{array}{c}\text { Kulacki and Emara [A-13] } \\
\text { Experimental }\end{array}$ \\
\hline 自 & $\begin{array}{c}\mathrm{Nu}_{\mathrm{u}}=0.360 \mathrm{Ra}^{0.223} \\
\mathrm{Nu}_{\mathrm{u}}=0.412 \mathrm{Ra}^{0.210} \dot{\mathrm{Pr}}^{0.041}\end{array}$ & $1 \times 10^{4}$ to $1 \times 10^{9}$ & $\begin{array}{c}6.5 \\
0.05 \text { to } 20\end{array}$ & $\ll 1$ & $\begin{array}{c}\text { Emara and Kulacki [A-14] } \\
\text { Numerical }\end{array}$ \\
\hline
\end{tabular}


Table A-2. Summary of experimental and numerical natural convection studies reviewed for rectangular cavities. (Continued)

\begin{tabular}{|c|c|c|c|c|c|}
\hline Geometry & Correlation & $\mathrm{Ra}^{\mathrm{a}}$ & $\operatorname{Pr}$ & $L / D$ & $\begin{array}{l}\text { Reference, } \\
\text { Type of Data }\end{array}$ \\
\hline & $\begin{array}{c}\mathrm{Nu}_{\mathrm{u}}=0.828 \mathrm{Ra}^{0.182} \\
\mathrm{Nu}_{\mathrm{u}}=0.759 \mathrm{Ra}^{0.178} \mathrm{Pr}^{0.059}\end{array}$ & $1 \times 10^{5}$ to $1 \times 10^{9}$ & $\begin{array}{c}6.5 \\
0.05 \text { to } 20\end{array}$ & $\ll 1$ & $\begin{array}{c}\text { Emara and Kulacki [A-14] } \\
\text { Numerical }\end{array}$ \\
\hline 1 & $\begin{aligned} \mathrm{Nu}_{s} & =0.6 \mathrm{Ra}^{0.19} \\
\mathrm{Nu}_{\mathrm{u}} & =0.345 \mathrm{Ra}^{0.233} \\
\mathrm{Nu}_{\mathrm{d}} & =1.389 \mathrm{Ra}^{0.095} \\
\mathrm{Nu}_{\mathrm{s}} & =0.85 \mathrm{Ra}^{0.19}\end{aligned}$ & $\begin{array}{l}1 \times 10^{7} \text { to } 3 \times 10^{10} \\
8 \times 10^{12} \text { to } 4 \times 10^{13}\end{array}$ & 7 & $<0.5$ & $\begin{array}{c}\text { Mayinger, Jahn, Reineke, } \\
\text { and Steinbrenner }[A-9, A-10] \\
\text { Experimental } \\
\text { Steinberner and Reineke } \\
\text { [A-11] } \\
\text { Experimental }{ }^{b}\end{array}$ \\
\hline
\end{tabular}

a. Where the Nusselt and Rayleigh numbers are defined as $N u=\frac{h L}{k}$ and $R a=\frac{g\left(q^{n+1} L^{s}\right.}{\alpha v k}$

b. Results were also applicable to a case with an upper and lower adiabatic boundary condition and constant temperature vertical walls. 


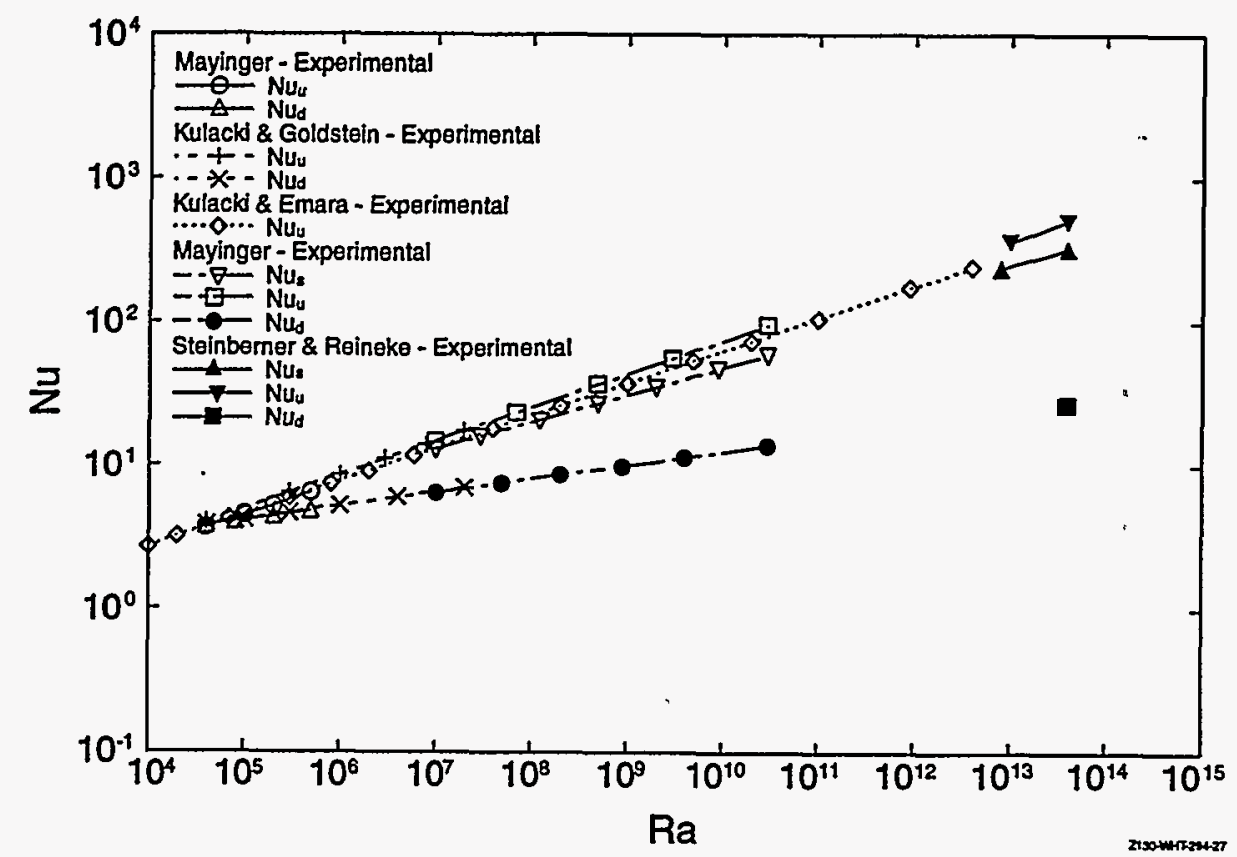

Figure A-4. Graphical comparison of correlations for rectangular layers and cavities based on experimental data.

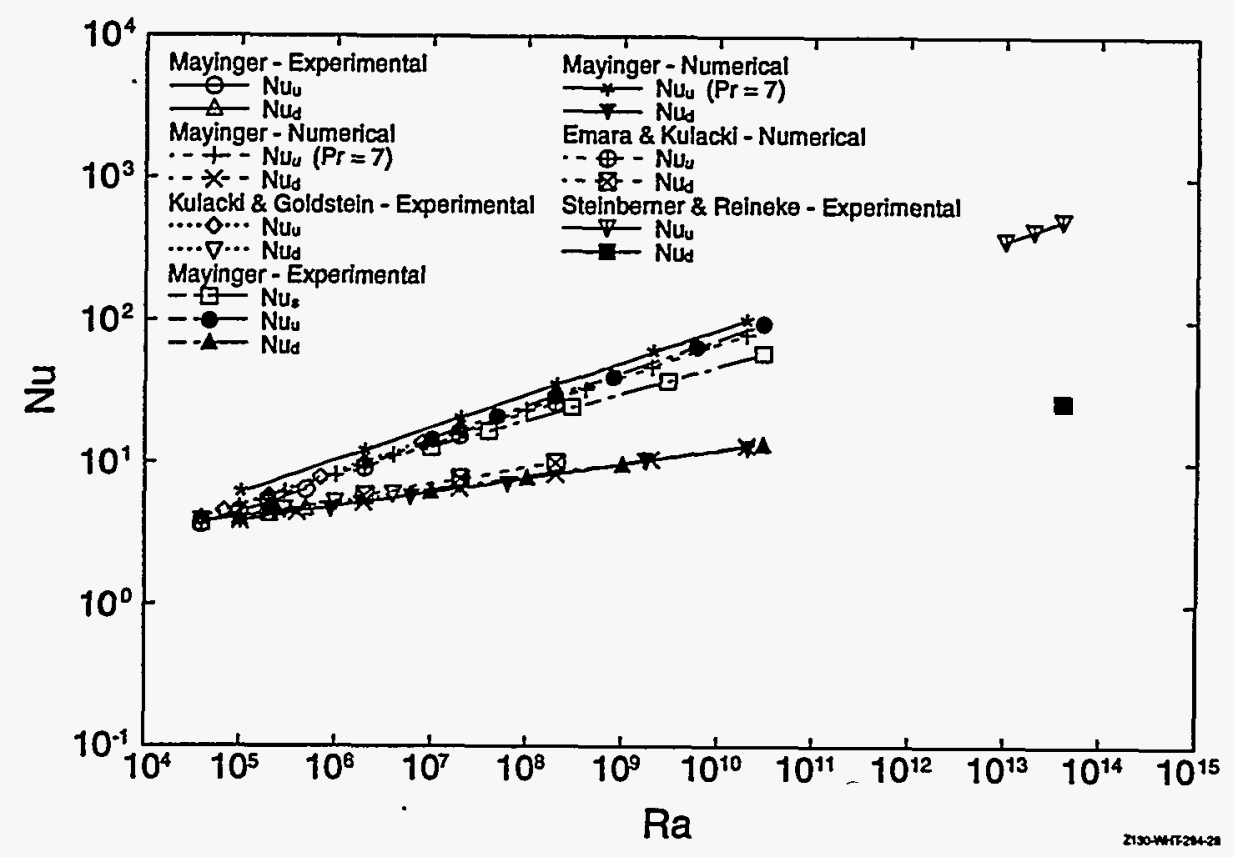

Figure A-5. Graphical comparison of correlations for rectangular layers and cavities having adiabatic side surfaces and constant temperature upper and lower surfaces. 


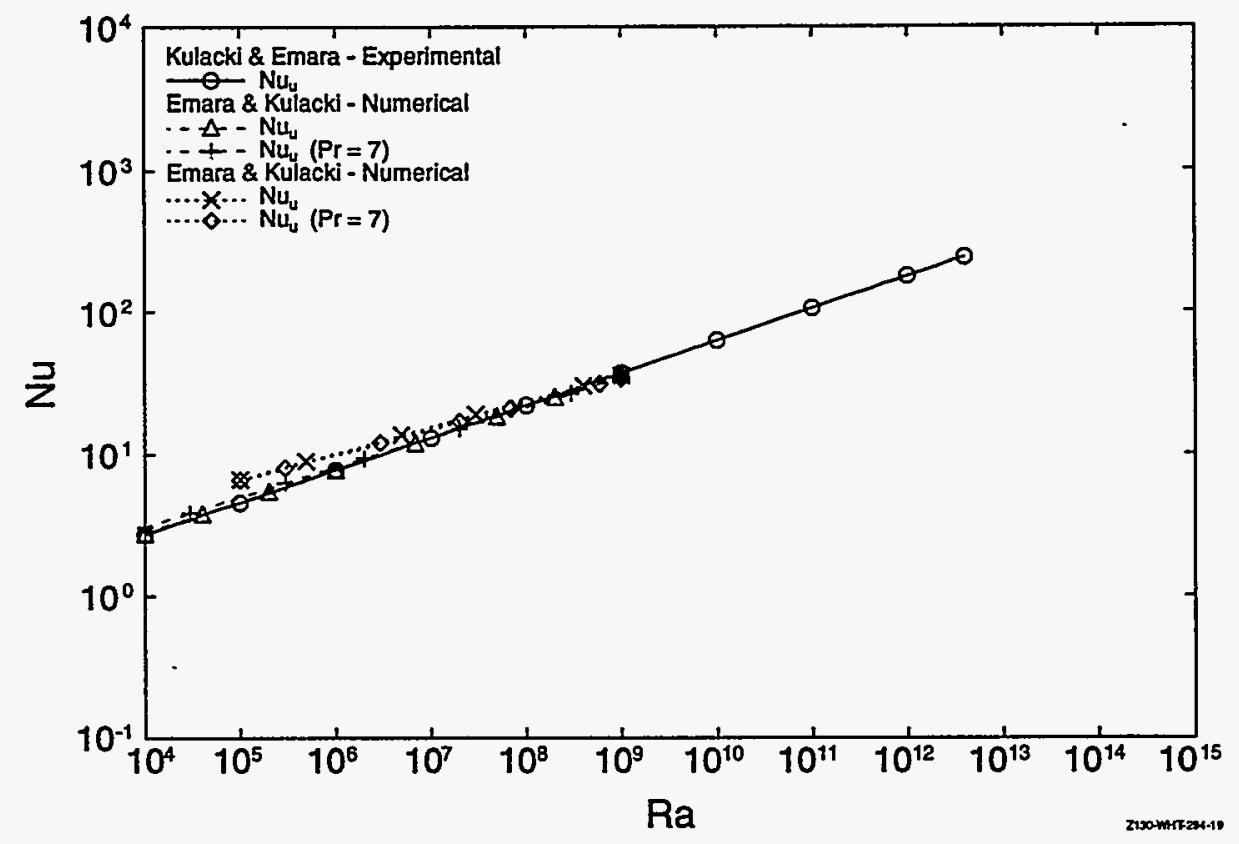

Figure A-6. Graphical comparison of correlations for rectangular layers and cavities having adiabatic side and lower surfaces and constant temperature upper surfaces.

\section{A2.1.1 Mayinger, Jahn, Reineke, and Steinbrenner}

Reference A-5, Reference A-9, and Reference A-10 summarize results from experimental and numerical investigations of natural convection in a fluid with uniform and constant volumetric heating. Experiments were performed in flat, thin, rectangular and semicircular cavities of various sizes. Numerical studies were performed for rectangular, semicircular, hemispherical, and cylindrical geometries. Results for the rectangular geometry studies are discussed in this section.

For the rectangular geometry experiments, uniform heating within the test fluid, water, was simulated by passing current between the top and bottom cooling channels of the cavity, which served as electrodes. Rayleigh numbers between $4 \times 10^{5}$ and $5 \times 10^{10}$ and a Prandtl number of 7 were investigated. Aspect ratios of 0.5 and less were investigated, although no effects of cavity dimensions were reported in the results. Steady state heat transfer results for the layer with constant equal temperature boundaries on the upper and lower walls and adiabatic boundaries on the side walls were found to be correlated by

$\mathrm{Nu}_{\mathrm{u}}=0.345 \mathrm{Ra}^{0.223}$

$\mathrm{Nu}_{\mathrm{d}}=1.389 \mathrm{Ra}^{0.095}$ 
For the layer with cooled side walls, data were obtained for water with Prandtl number of 7, and Rayleigh numbers from $10^{7}$ to $3 \times 10^{10}$ in cavities with aspect ratios less 0.5 . .

$N u_{s}=0.6 R a^{0.19}$
$N_{u}=0.345 \mathrm{Ra}^{0.233}$
$N u_{d}=1.389 \mathrm{Ra}^{0.095}$.

Reference A-5 discusses the uncertainty associated with these experiments. It is noted that there are errors due to optical uncertainties, uncertainties in thermo-physical properties, and geometrical quantities. The maximum error in the Nusselt numbers measured in these experiments is estimated as $15 \%$, consisting of uncertainties in measuring the temperature difference $(\leq 5 \%)$, the heat flux at the boundary $(-5 \%)$, and the thickness of the layer $(\leq 5 \%)$. The maximum error in the Rayleigh number is estimated to range from 5 to $25 \%$, consisting of uncertainty in measuring the power input $(\leq 1 \%)$, the layer thickness $(\leq 5 \%)$, and the thermal properties of the test fluid $(\leq 2 \%)$, Note that the somewhat large upper estimate for error in the Rayleigh number is attributed to a relatively large uncertainty in the layer thickness, particularly at very low Rayleigh number where thin layers were studied. Generally, Reference A-5 estimates that the average error in the Rayleigh and Nusselt numbers is around $10 \%$.

Numerical studies were performed using the THEKAR R computer program. ${ }^{A-9}$ Calculations were performed for horizontal layers with aspect ratios of 0.5 , assuming that the horizontal boundaries were at constant and equal temperature and that the vertical boundaries were adiabatic. Although computations were also performed for layers with smaller aspect ratios, it was reported that aspect ratio had little effect on the heat transfer results. Studies were also performed for horizontal layers with a free upper surface. Calculations were performed for Rayleigh numbers between $10^{5}$ to $2 \times 10^{10}$ and Prandtl numbers between 0.04 and 10. Correlations obtained for these geometries are summarized below.

$\mathrm{Nu}_{\mathrm{u} \text { (rigid) }}=0.292 \mathrm{Ra}^{0.23} \operatorname{Pr}^{0.085}$

$\mathrm{Nu}_{\mathrm{u} \text { (free) }}=0.368 \mathrm{Ra}^{0.23} \operatorname{Pr}^{0.085}$

$\mathrm{Nu}_{\mathrm{d}}=1.235 \mathrm{Ra}^{0.10}$

In reviewing the work reported in Reference A-9 and Reference A-10, Reference A-5 noted that the numerically-obtained correlations were calculated using a uniform grid distribution without using any turbulence model. Although results obtained for the weakly turbulent regime $\left(10^{5} \leq \mathrm{Ra} \leq 10^{7}\right)$ and a Prandtl number of 7 were found to agree qualitatively with the experimental work, Reference A-5 questioned whether results could be extrapolated to higher Rayleigh number regimes where the flow and 
temperature fields are characterized by thin boundary layers near the surfaces, a well mixed isothermal core region and turbulent mixing at the wall-core region interface.

\section{A2.1.2 Steinberner and Reineke}

Reference A-11 reports results from an experimental and a numerical investigation of natural convection in a fluid with internal heat sources in a closed rectangular cavity for Rayleigh numbers up to $3.7 \times 10^{13}$. In these experiments, water was contained in a flat rectangular cavity. A uniform volumetric heat source was simulated using Joulean heating by passing an alternating current though the fluid. The cavity was $0.8 \mathrm{~m}$ long and $0.035 \mathrm{~m}$ deep and wide.

To investigate heat transfer to vertical walls, two types of boundary conditions were simulated: cases with constant temperature vertical walls of the rectangular cavity and adiabatic top and bottom walls of the cavity; and cases with cooling on all walls of the rectangular cavity. For each case, Rayleigh numbers were varied from $8.4 \times 10^{12}$ to $3.7 \times 10^{13}$. For both of the boundary conditions investigated, results indicate that the heat transfer for the vertical walls can be described using the following relationship

$\mathrm{Nu}_{\mathrm{s}}=0.85 \mathrm{Ra}^{0.19}$

As depicted in Figure A-7, this relationship was found to be in good agreement with relationships obtained by Mayinger et al. ${ }^{A-9}$ Reference A-11 contains plots comparing the local Nusselt numbers as a function of height along the side of the cavity. Results indicate that the heat flux density has a distinct maximum at the upper part of the vertical wall and decreases towards the bottom. The maximum local Nusselt numbers are approximately twice as high as the average Nusselt numbers predicted above with Equation (A-9).

Reference A-11 also reports a limited number of data obtained for experiments with upper and lower boundaries cooled and adiabatic vertical walls. The limited data obtained from these experiments indicated that upward and downward heat transfer could be modeled using the relations below, which were originally obtained by Jahn and Reineke in Reference A-10.

$\mathrm{Nu}_{\mathrm{u}}=0.345 \mathrm{Ra}^{0.233}$

$\mathrm{Nu}_{\mathrm{d}}=1.389 \mathrm{Ra}^{0.095}$

However, it should be noted that the data obtained for predicting heat transfer upward and downward was more limited. For example, only one data point was obtained for modeling downward heat transfer.

\section{A2.1.3 Kulacki and Goldstein}

Reference A-12 reports results from an experimental investigation of natural convection in a rectangular cavity containing aqueous silver nitrate solution with volumetric heat sources. The upper and 
lower surfaces of the convection chamber, which consisted of copper plates, were held constant with thermostatically controlled water circulating through a double spiral channel brass plate bolted to the back of each copper plate. Vertical surfaces of the cavity were designed to be adiabatic. Volumetric energy sources were simulated in the fluid by using Joule heating with a $60 \mathrm{~Hz}$ alternating current.

The study was performed to investigate the initial flow instability, the nature of the flow and mean temperature fields in the post-stability regimes, and the heat transport within the layer and at its boundaries. Data were obtained for Rayleigh numbers between $3.7 \times 10^{4}$ and $2.4 \times 10^{7}$, Prandtl numbers between 5.7 and 6.4, and cavities with aspect ratios between 0.05 and 0.25 . Average values of the Nusselt number were found to correlate with the Rayleigh numbers using the following equations

$$
\begin{aligned}
& \mathrm{Nu}_{\mathrm{u}}=0.329 \mathrm{Ra}^{0.236} \\
& \mathrm{Nu}_{\mathrm{d}}=1.43 \mathrm{Ra}^{0.094} .
\end{aligned}
$$

Temperature distributions from these experiments were used to define three approximate flow regimes: (a) gentle laminar convection for $\mathrm{Ra} \leq 3 \times 10^{5}$, where conduction dominates heat transfer; (b) fully-developed laminar convection for $3 \times 10^{5} \leq \mathrm{Ra} \leq 6 \times 10^{6}$; and (c) a region where the transition to turbulent convection occurs for approximately $\operatorname{Ra}>6 \times 10^{6}$.

In Reference A-12, the authors estimate that the combined uncertainty in their experimental results ranges from 2 to $3 \%$ and the total experimental uncertainty in the computed values of the Nusselt number ranges from 3 to $7 \%$. These estimates includes uncertainty in thermophysical properties, geometrical factors, and wattmeter readings (which were used to estimate the total power in the convection cell).

\section{A2.1.4 Kulacki and Emara}

In Reference A-13, Kulacki and Emara document results from an experimental investigation of steady and transient thermal convection in a rectangular cavity containing aqueous silver nitrate solution with volumetric heat sources. The layer was bounded below by a rigid adiabatic surface and above by a rigid isothermal surface. The top plate contained channels for circulating cooling water that was maintained at a constant temperature. A sheet of Mylar covered the top plate to insulate it electrically from the fluid layer. Two of the side walls contained silver-plated electrodes for passing a $60 \mathrm{~Hz}$ alternating electric current through the fluid. The other side walls and the lower surface were Plexiglas. Because the layers were thin relative to their horizontal dimension, edge effects and secondary flow effects were minimized. Thermocouples were used to measure temperatures across the layer. The response of the thermocouples were fairly rapid ( $<1 \mathrm{~s}$ for a 1 degree $\mathrm{K}$ step input) so that transient temperature behavior could be measured. Experiments were performed to investigate the steady-state heat transfer from the fluid to the upper surface and to investigate the time required for a layer to reach steady state as a function of step change in Rayleigh number. Results from the steady-state experiments are discussed below; results from the transient experiments are discussed in Section A3.1.1.

Data obtained from the steady-state experiments were correlated by 
$\mathrm{Nu}_{\mathrm{u}}=0.338 \mathrm{Ra}^{0.227}$

for fluids with Prandtl number between 2.8 and 6.9 and Rayleigh number between $4 \times 10^{3}$ and $4 \times 10^{12}$. Aspect ratios investigated in the steady-state experiments ranged from 0.025 to 0.5 . No attempt to correlate the data for Prandtl number effects was made because most of the data were obtained for a Prandtl number of 6.5 .

In Reference A-13, the authors indicate that the experimental uncertainty in the Rayleigh number is between 5 and $7 \%$. This estimate represents the combined uncertainty in the thermophysical properties of the aqueous silver nitrate solutions, geometrical factors (primarily the depth of the layer), and the power consumption per unit volume. The uncertainty in the steady-state Nusselt number is estimated as between 4 and $5 \%$.

\section{A2.1.5 Emara and Kulacki}

Reference A-14 reports results from a numerical study to predict heat transfer from a fluid with volumetric heat sources in a rectangular cavity. In this study, it was assumed that the side walls of the layer were adiabatic surfaces. Calculations were performed for three cases: cases assuming that the upper and lower surfaces were rigid and at constant temperature; cases assuming that the upper surface was rigid and at constant temperature and the lower surface was rigid and adiabatic; and cases assuming that the upper surface was free and at constant temperature and the lower surface was rigid and adiabatic.

For a layer with upper and lower surfaces at constant temperature, the calculations indicate that heat transfer from the fluid to the surfaces can be predicted using

$\mathrm{Nu}_{\mathrm{u}}=0.328 \mathrm{Ra}^{0.23}$

$\mathrm{Nu}_{\mathrm{d}}=1.041 \mathrm{Ra}^{0.119}$

for Rayleigh numbers between $2 \times 10^{5}$ and $2 \times 10^{8}$ and fluids with Prandtl number of 6.5 .

For the calculations assuming an insulated lower boundary, results indicate that the heat transfer from a fluid with a rigid isothermal upper surface and Rayleigh numbers between $1 \times 10^{4}$ and $1 \times 10^{9}$ can be modeled using

$\mathrm{Nu}_{\mathrm{u}}=0.360 \mathrm{Ra}^{0.223}$

for fluids with a Prandtl number of 6.5. Calculations performed for fluids with Prandtl numbers between 0.05 and 20 indicate that Prandtl number effects can be incorporated using 
$\mathrm{Nu}_{\mathrm{u}}=0.412 \mathrm{Ra}^{0.210} \mathrm{Pr}^{0.041}$

Calculation results indicate that the heat transfer from a fluid with a free isothermal upper surface and Rayleigh numbers between $1 \times 10^{5}$ and $1 \times 10^{9}$ can be modeled using

$\mathrm{Nu}_{\mathrm{u}}=0.828 \mathrm{Ra}^{0.182}$

for fluids with a Prandtl number of 6,5. Calculations performed for fluids with Prandtl numbers between 0.05 and 20 indicate that Prandtl number effects can be incorporated using

$N u_{u}=0.759 \mathrm{Ra}^{0.178} \operatorname{Pr}^{0.059}$

Calculation results for the rigid, isothermal upper boundary were compared with the experimental data obtained by Kulacki and Emara ${ }^{\mathrm{A}-13}$ and found to be in good agreement for the range of Rayleigh numbers for which these calculations were performed.

\section{A2.2 Curved Geometries}

Experimental and numerical studies have been performed to investigate heat transfer from molten pools with volumetric heat sources in hemispherical, spherical, and torispherical cavities. Key studies performed for these geometries are summarized below. Table A-3 summarizes the studies reviewed in this section. Correlations developed for predicting heat transfer are graphically compared in Figure A-10 through Figure A-12. Figure A-10 compares correlations obtained from two-dimensional numerical and experimental studies. Figure A-11 compares correlations obtained from three-dimensional geometries with hemispherical lower surfaces. Figure A-12 compares correlations obtained from three-dimensional cylindrical and torispherical geometries.

As illustrated in these figures, the Rayleigh numbers investigated in some of these experiments are higher than Rayleigh numbers investigated in experiments discussed in Section A2.1. Several tests were performed for Rayleigh numbers above $10^{12}$. However, the peak Rayleigh numbers investigated were limited to $10^{15}$, which is still lower than the upper bound of $10^{17}$ estimated for severe accident conditions. Likewise, the data discussed within this section were primarily obtained using aqueous solutions with Prandtl numbers of 7.0 instead of the lower values expected for fluids in molten pools that would form during a severe accident.

Data in Figure A-10 through Figure A-12 exhibit considerably more scatter than data reviewed in Section A2.1. Although most correlations indicate that the heat transfer toward the upper boundary is higher than toward the lower boundary, the correlations proposed by Mayinger ${ }^{A-9}$ suggest that heat transfer to the upper boundary is higher. There is also discrepancy about the effects of the test facility's height to radius ratio. Some correlations indicate that the height to radius ratio impacts results, whereas other tests indicate that this ratio did not impact heat transfer. Note that the independence of heat transfer 
with the length scale of the facility is consistent with the $\sim 0.2$ exponent recommended in most of the heat transfer correlations proposed by studies reviewed in Section A2.1 and Section A2.2.

Although there is a large amount of uncertainty associated with this data, it is currently recommended that the correlations proposed by Kymäläinen, et al., are most applicable for severe accident conditions. These correlations were based on tests performed at higher Rayleigh numbers and appear consistent with correlations obtained from many of the other experiments. Nevertheless, the data reviewed in this section indicates that there is still considerable uncertainty in modeling the heat transfer from a fluid with uniform heating. In particular, information presented in this section indicates that additional research is needed to investigate possible three-dimensional effects on heat transfer that may not have been observed in the "slice" geometry used for the Kymäläinen, et al. COPO tests.

Additional recommendations based on the data reported in Section A2.1 and A3.2 may be found in Section A2.3. Graphical comparisons of correlations presented in these two sections are also found in Section A2.3. 
Table A-3. Summary of experimental and numerical natural convection studies reviewed for spherical, hemispherical, and torispherical cavities.

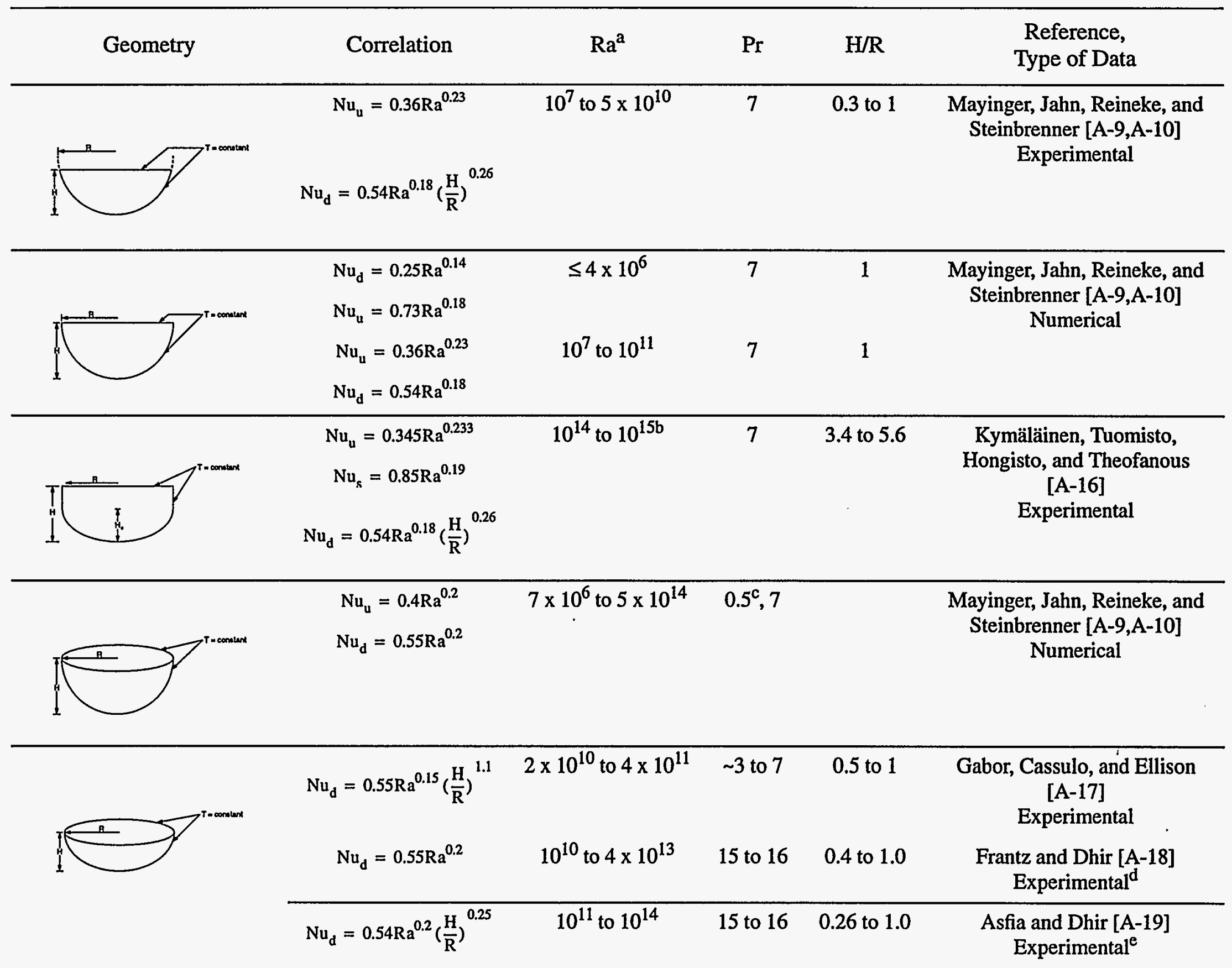


Table A-3. Summary of experimental and numerical natural convection studies reviewed for spherical, hemispherical, and torispherical cavities.

\begin{tabular}{|c|c|c|c|c|c|}
\hline Geometry & Correlation & $\mathrm{Ra}^{\mathrm{a}}$ & $\operatorname{Pr}$ & $\mathrm{H} / \mathrm{R}$ & $\begin{array}{l}\text { Reference, } \\
\text { Type of Data }\end{array}$ \\
\hline$B$ & $\begin{aligned} \mathrm{Nu}_{\mathrm{u}} & =0.34 \mathrm{Ra}^{0.22} \\
\mathrm{Nu}_{\mathrm{u}} & =2.8 \mathrm{Ra}^{0.09} \\
\mathrm{Nu}_{\mathrm{d}} & =0.935 \mathrm{Ra}^{0.10} \\
\mathrm{Nu}_{\mathrm{s}} & =0.495 \mathrm{Ra}^{0.18}\end{aligned}$ & $\begin{array}{c}10^{5} \text { to } 2 \times 10^{7 f} \\
2 \times 10^{7} \text { to } 10^{9} \\
10^{5} \text { to } 10^{9} \\
10^{5} \text { to } 10^{9}\end{array}$ & 7 & 2 & $\begin{array}{c}\text { Mayinger, Jahn, Reineke, and } \\
\text { Steinbrenner [A-9,A-10] } \\
\text { Numerical }\end{array}$ \\
\hline \multirow[t]{3}{*}{ P } & $\mathrm{Nu}_{\mathrm{u}}=2.086 \mathrm{Ra}^{0.127}$ & $9 \times 10^{7}$ to $1 \times 10^{10 g}$ & $\begin{array}{l}2.1 \text { to } \\
5.9\end{array}$ & $0.22^{h}$ & $\begin{array}{c}\text { Min and Kulacki [A-15] } \\
\text { Experimental }\end{array}$ \\
\hline & $\begin{array}{l}\mathrm{Nu}_{\mathrm{u}}=2.320 \mathrm{Ra}^{0.125} \\
\mathrm{Nu}_{\mathrm{u}}=1.091 \mathrm{Ra}^{0.166}\end{array}$ & $\begin{array}{l}3 \times 10^{8} \text { to } 3 \times 10^{11} \\
4 \times 10^{10} \text { to } 1 \times 10^{13}\end{array}$ & $\begin{array}{c}2.7 \text { to } 7 \\
2.3 \text { to } \\
6.8\end{array}$ & $\begin{array}{c}0.30 \text { to } 0.32 \\
0.81\end{array}$ & \\
\hline & $\mathrm{Nu}_{\mathrm{u}}=0.280 \mathrm{Ra}^{0.227}$ & $6 \times 10^{11}$ to $2 \times 10^{14}$ & $\begin{array}{l}1.9 \text { to } \\
5.8\end{array}$ & 1.37 to 1.41 & \\
\hline
\end{tabular}

b. For the downward heat transfer case in this study, the Nusselt and Rayleigh numbers are defined as $\mathrm{Nu}=\frac{\mathrm{hH}}{\mathrm{k}}$ and $\mathrm{Ra}=\frac{\mathrm{g} \beta \mathrm{q} " \mathrm{H}_{\mathrm{c}}^{\mathrm{\prime}}}{\alpha \nu \mathrm{k}}$

c. Evaluated for $\mathrm{Ra}=7 \times 10^{6}$.

d. Upper surface of pool in these experiments was exposed to air.

e. Three types of pool upper surface boundary conditions were tested: a free surface, a rigid insulated surface, and a rigid cooled surface.

f. For all cases in this study, the Nusselt and Rayleigh numbers are defined as $\mathrm{Nu}=\frac{\mathrm{hH}}{\mathrm{k}}$ and $\mathrm{Ra}=\frac{\mathrm{g} \beta \mathrm{q} " \mathrm{\prime \prime}}{\alpha v \mathrm{k}}$

g. For all cases in this study, the Nusselt and Rayleigh numbers are defined as $\mathrm{Nu}=\frac{\mathrm{hH}}{\mathrm{k}}$ and $\mathrm{Ra}=\frac{\mathrm{g} \beta \mathrm{q}^{\prime \prime} \mathrm{H}^{5}}{\alpha \mathrm{k}}$

h. In this case, the pool height was below the junction between the curved bottom and the cylindrical sidewall. 


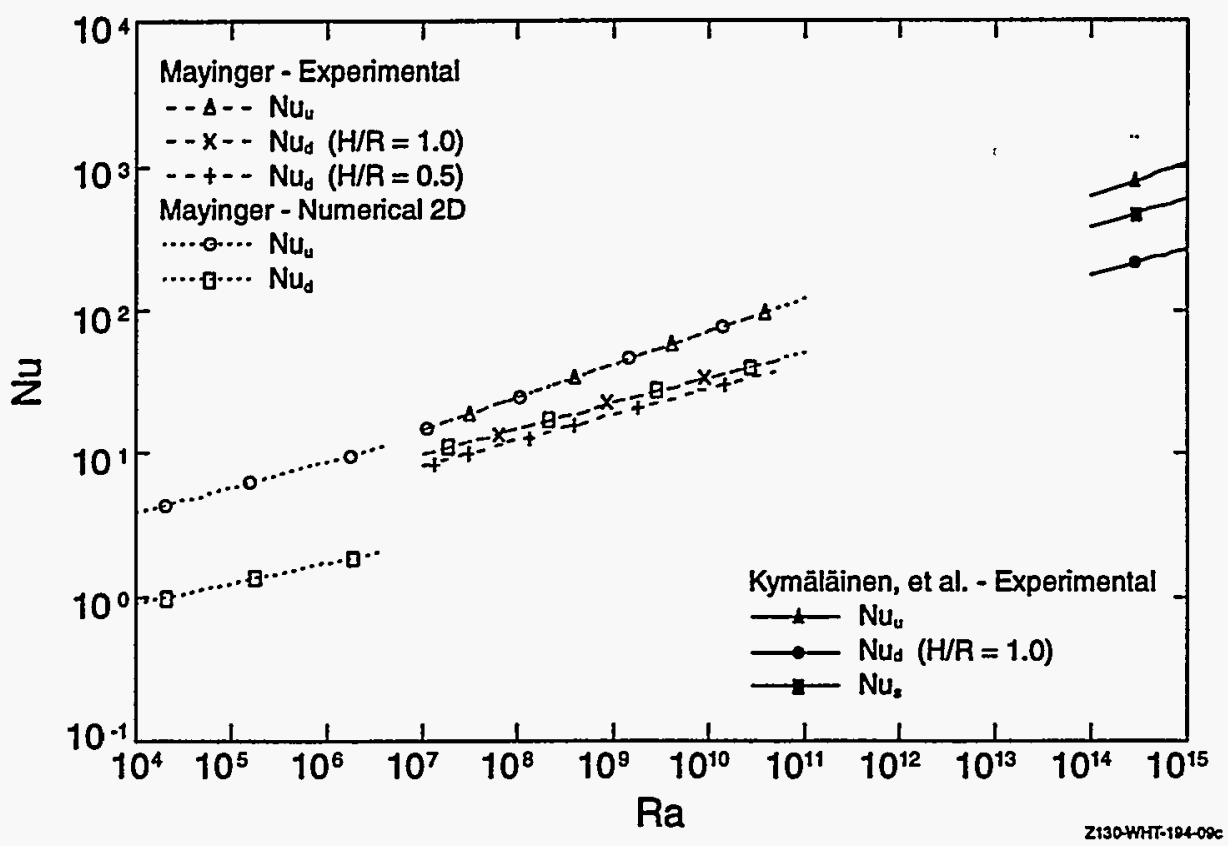

Figure A-7. Graphical comparison of numerically and experimentally-based correlations for curved two-dimensional "slice" geometries.

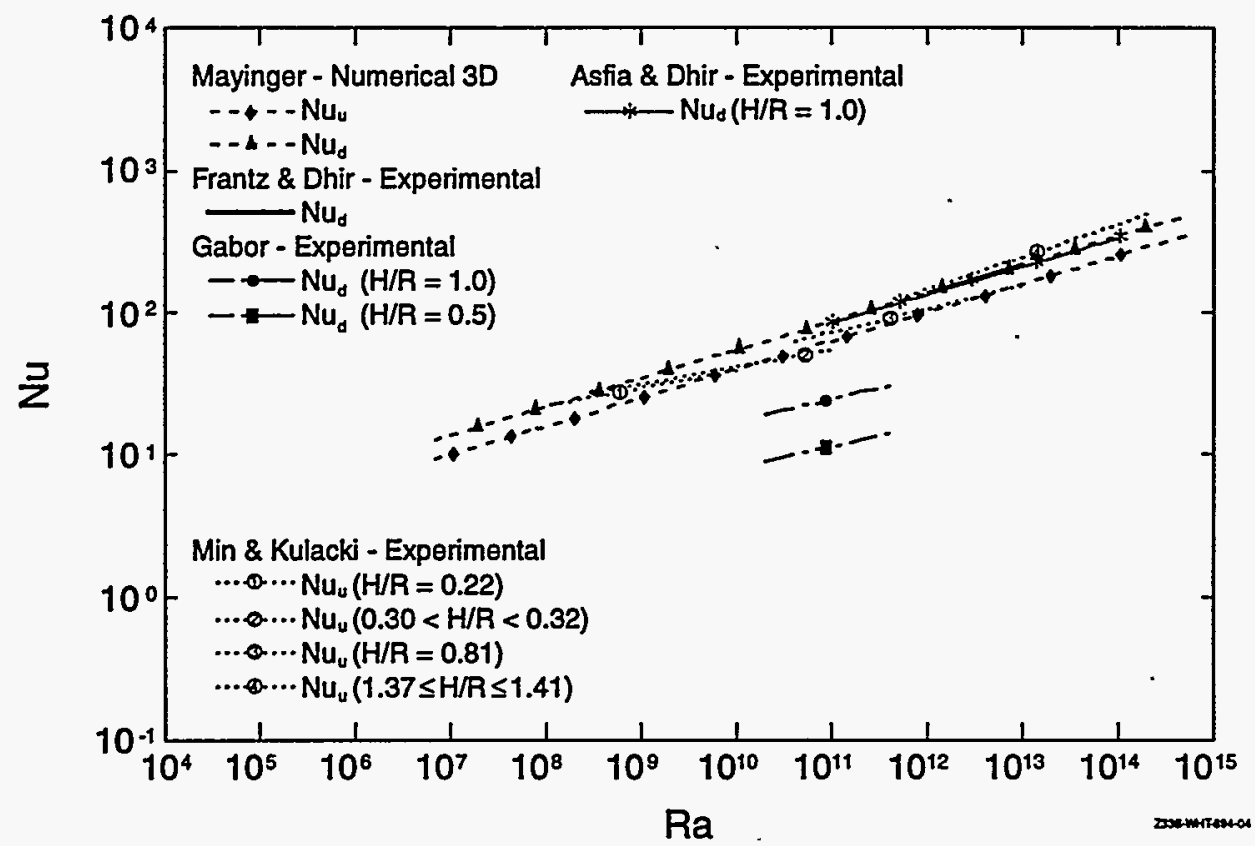

Figure A-8. Graphical comparison of numerically and experimentally-based correlations for curved three-dimensional geometries. 


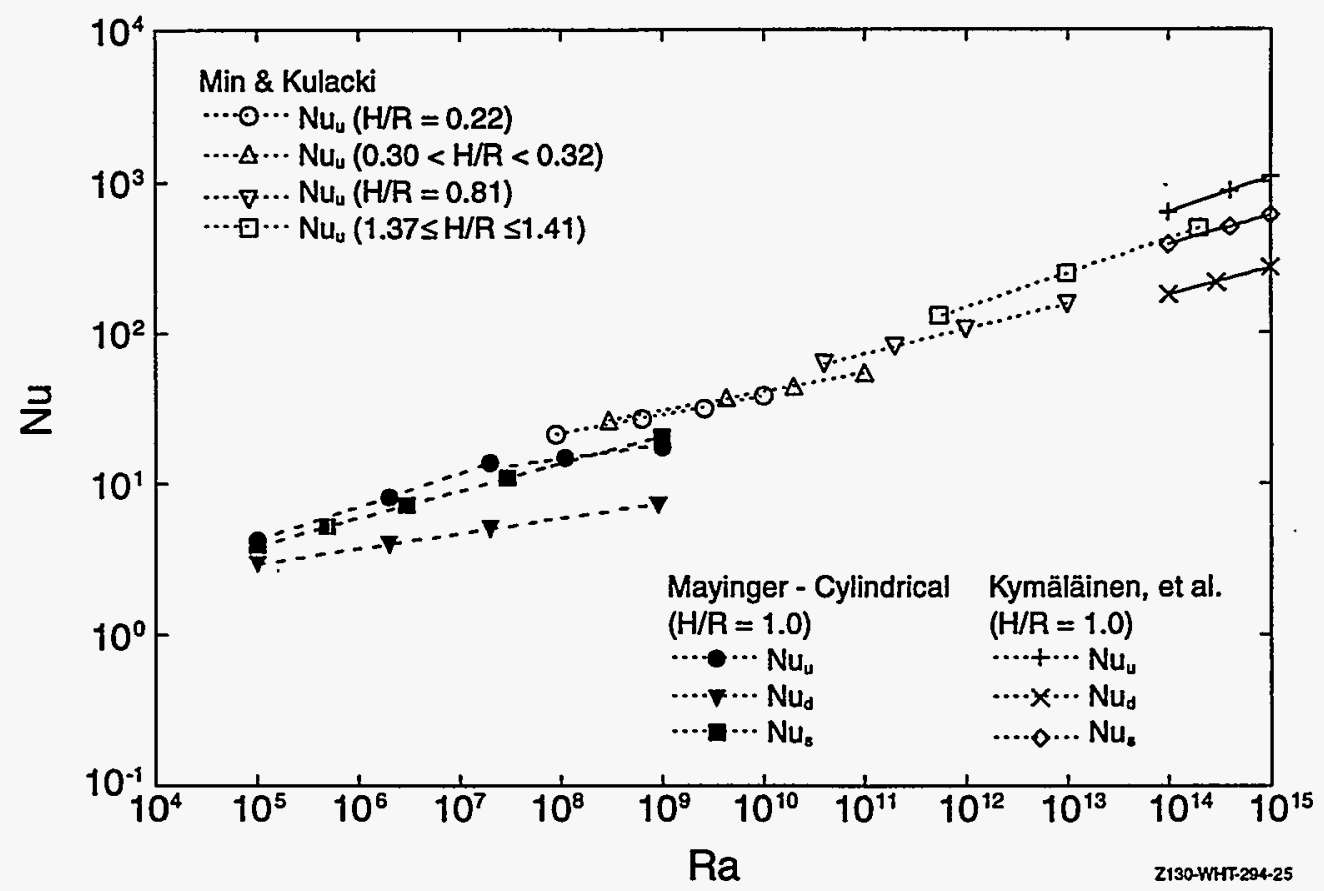

Figure A-9. Graphical comparison of correlations for curved three-dimensional cylindrical/torispherical geometries

\section{A2.2.1 Mayinger, Jahn, Reineke, and Steinbrenner}

References A-5, A-9, and A-10 summarize results from experimental and numerical investigations of natural convection in a fluid with uniform and constant volumetric heating. Experiments were performed in flat, thin, rectangular and semicircular cavities of various sizes. Numerical studies were performed for rectangular, semicircular, hemispherical, and cylindrical geometries. Results for spherical, cylindrical and hemispherical geometry studies are discussed in this section.

Experiments were performed in a thin, semicircular or circular segment, two dimensional test cell containing water. In order to obtain electrodes with uniform spacing in this geometry, the sides of the test cell, which must be transparent in order to allow recording of the interference picture, were used as the electrodes. Hence, the glass plates bounding the sides of the test cavity were coated with a layer of $\mathrm{SnO}_{2}$. Upper and lower boundaries of the test cell were electrically insulated metal plates which contained channels for circulation of cooling water. The radius of the semicircle was varied from 0.025 to 0.280 and the height of the circular segment (at the centerline) was varied from $0.3 \mathrm{R}$ to $\mathrm{R}$. It should be noted that results from this "slice" test have been applied in many severe accident analyses and are currently the basis for the molten pool heat transfer model in SCDAP/RELAP5/MOD3.1 (see Section A1.3).

For the semicircular, two-dimensional test cell, the following heat transfer correlations were obtained:

$\mathrm{Nu}_{\mathrm{u}}=0.36 \mathrm{Ra}^{0.23}$ 
$\mathrm{Nu}_{\mathrm{d}}=0.54 \mathrm{Ra}^{0.18}$

for $10^{7} \leq \mathrm{Ra} \leq 5 \times 10^{10}$ and a Prandtl number of 7 . Note that both the Nusselt number and the Rayleigh number are defined in terms of the radius of the semicircle. As discussed above in Section A2.2.1, the average experimental error in the Nusselt and Rayleigh numbers is estimated as approximately $10 \%$.

In these experiments, it was noted that there were three different areas of flow behavior. On the upper wall, a non-uniform eddy flow was observed. In the lower part of the cavity, a stable, calm liquid layer formed. In the upper half of the curved bottom, isotherm patterns typical of a cooled vertical wall occurred. Hence it was concluded that the Nusselt number varied considerably across the cooled surface and the local distribution of heat transfer on the semicircular segment was measured. Results, which are plotted in Figure A-13, indicated that the local Nusselt number at the top of the cavity may be over twice as large as the average Nusselt number.

Thus, for an example case with a Rayleigh number of $10^{10}$, the average upward and downward Nusselt numbers are 71.8 and 34.1, respectively. Applying the experimental curve shown in Figure A-13 to the average downward Nusselt number of 34.1, this yields a local Nusselt number of 6.8 at the bottom of the hemisphere ( 0 degrees), a Nusselt number of 32.7 at an angle of 45 degrees from the bottom, and a Nusselt number of 66.8 at the top (90 degrees). Thus, the curve shown in Figure A-13 gives a fairly continuous distribution for the local Nusselt numbers with values that are fairly consistent with the average Nusselt numbers predicted by Equations (A-29) and (A-30). Additional discussion about the variation in local heat flux along the bottom surface of various test facilities may be found in Section A2.3.

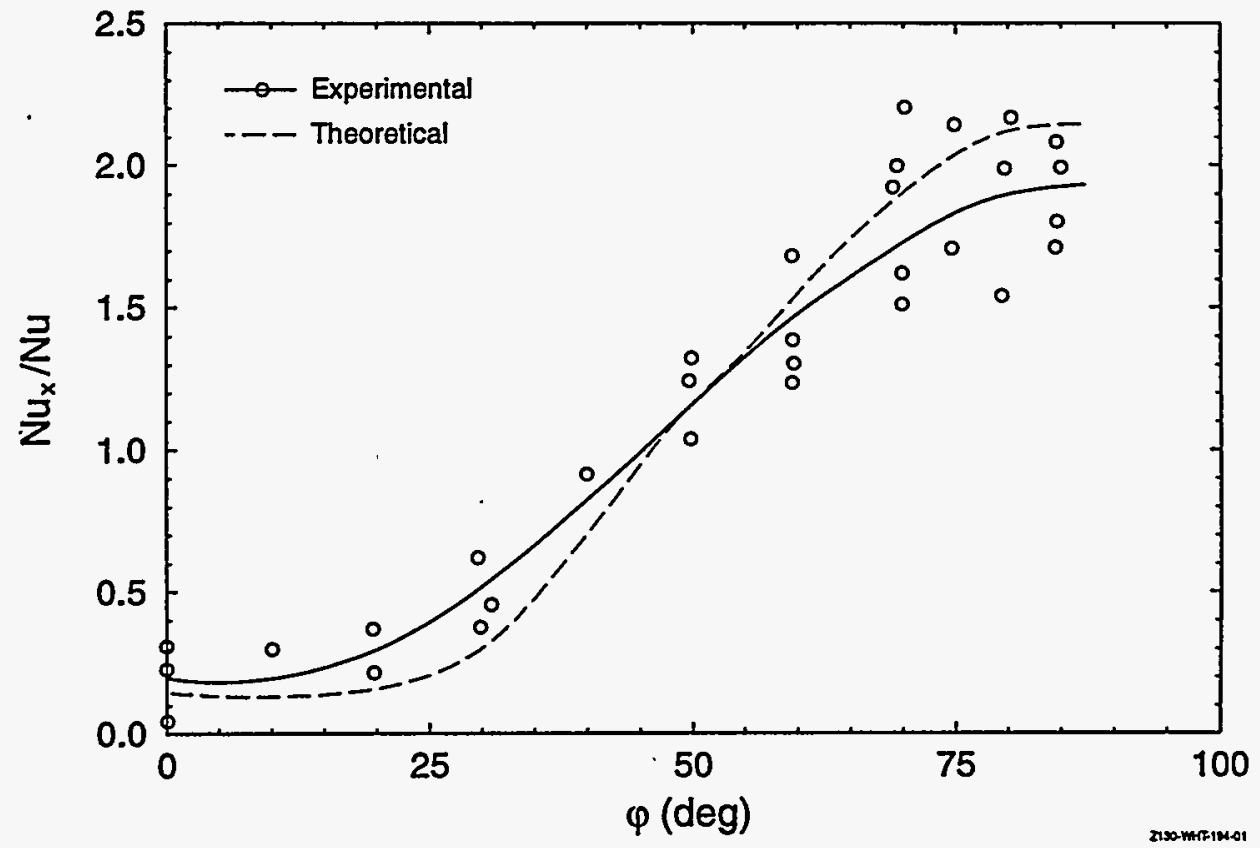

Figure A-10. Ratio of local to average Nusselt number for a semicircular cavity ${ }^{\mathrm{A}-10}$.

For the circular segment test cell, the following heat transfer correlations were obtained for fluids with Rayleigh numbers between $10^{7}$ and $5 \times 10^{10}$ and a Prandtl number of 7: 
$\mathrm{Nu}_{\mathrm{u}}=0.36 \mathrm{Ra}^{0.23}$

$\mathrm{Nu}_{\mathrm{d}}=0.54 \mathrm{Ra}^{0.18}\left(\frac{\mathrm{H}}{\mathrm{R}}\right)^{0.26}$

where the Nusselt and Rayleigh numbers are defined in terms of $R$, and $0.5 \leq H / R \leq 1.0$.

For a cylindrical geometry, numerical studies were performed using the THEKAR $R$ computer program. ${ }^{\mathrm{A}-9}$ Analyses were performed assuming that all surfaces of the cylinder were at constant and equal temperatures. Rayleigh numbers between $10^{5}$ and $1.5 \times 10^{7}$ were considered for a fluid with Prandtl number of 7. Results indicate that heat transfer can be predicted using the following correlations:

$$
\mathrm{Nu}_{\mathrm{u}}=0.34 \mathrm{Ra}^{0.22}
$$

for $10^{5} \leq \mathrm{Ra} \leq 1.5 \times 10^{7}$ and Prandtl number of 7 ,

$\mathrm{Nu}_{\mathrm{u}}=2.8 \mathrm{Ra}^{0.09}$

for $2 \times 10^{7} \leq \mathrm{Ra} \leq 10^{9}$ and Prandtl number of 7 ,

$\mathrm{Nu}_{\mathrm{d}}=0.935 \mathrm{Ra}^{0.10}$

for $10^{5} \leq \mathrm{Ra} \leq 10^{9}$ and Prandtl number of 7 , and

$\mathrm{Nu}_{\mathrm{s}}=0.495 \mathrm{Ra}^{0.18}$

for $10^{5} \leq \mathrm{Ra} \leq 10^{9}$ and Prandtl number of 7. In Table A-3, it is noted that the characteristic length for the Rayleigh and Nusselt numbers is the height of the cylinder and that calculations assumed a right circular cylinder with a height to diameter ratio of 1.0.

In these calculations, a uniform grid was assumed and no turbulence model was included. As discussed above in Section A2.2.1, Reference A-5 noted that it is questionable if low Rayleigh number results could be extrapolated to higher Rayleigh number regimes where the flow and temperature fields are characterized by thin boundary layers near the surfaces, a well mixed isothermal core region and turbulent mixing at the wall-core region interface. 
For the two-dimensional semicircular slice and three-dimensional hemispherical geometries, numerical studies were performed using the THEKAR H1 and THEKAR H2 computer programs. ${ }^{\text {A-9 }}$ The THEKAR H1 version uses a uniform grid mesh with no turbulence model. For higher Rayleigh numbers, the $\mathrm{H} 2$ version of THEKAR was used because it includes a nonuniform grid mesh and a turbulence model. For a filled, two-dimensional, semicircular cavity with rigid surfaces at constant and equal temperatures, the following correlations were obtained for fluids with a Prandtl number of 7

$N u_{d}=0.25 \mathrm{Ra}^{0.14}$

$\mathrm{Nu}_{\mathrm{u}}=0.73 \mathrm{Ra}^{0.18}$

for fluids with Rayleigh number less than or equal to $4 \times 10^{6}$. For fluids with higher Rayleigh number $\left(10^{7}\right.$ $\leq \mathrm{Ra} \leq 10^{11}$ ), the following correlations were obtained

$\mathrm{Nu}_{\mathrm{u}}=0.36 \mathrm{Ra}^{0.23}$

$\mathrm{Nu}_{\mathrm{d}}=0.54 \mathrm{Ra}^{0.18}$

In these correlations, the cavity radius is the characteristic length in the Rayleigh and Nusselt numbers. Limited results are presented for the variation for the local Nusselt number on the semicircular floor. For a Rayleigh number of $10^{10}$, the local Nusselt number reaches a maximum at the edge of the cavity (for $\varphi=90^{\circ}$ ) which is about 10 to 15 times the value at the center (for $\varphi=0^{\circ}$ ).

For a filled, three-dimensional hemispherical cavity with rigid surfaces at constant and equal temperature, the following correlations were obtained.

$\mathrm{Nu}_{\mathrm{u}}=0.4 \mathrm{Ra}^{0.2}$

$\mathrm{Nu}_{\mathrm{d}}=0.55 \mathrm{Ra}^{0.2}$

where the length parameter in the Nusselt and Rayleigh number is the pool radius. The above correlations were obtained for a range of $7 \times 10^{6} \leq \mathrm{Ra} \leq 5 \times 10^{14}$ and a Prandtl number of 0.5 . It is noted in Reference A-5 that the local Nusselt number is a minimum at the center (for $\varphi=0^{\circ}$ ) and increases to a maximum near the upper edge (for $\varphi=90^{\circ}$ ). Edge values of the Nusselt number were found to be from 2 to 6 times the minimum value at the center, depending on Rayleigh number.

The above correlations exhibit a peculiar behavior compared to other correlations obtained for these geometries. Equations (A-41) and (A-42) suggest that the Nusselt number for heat transfer in the upward 
direction is smaller than the Nusselt number for heat transfer in the downward direction. As illustrated in Figure A-11, this contradicts correlations obtained for a two-dimensional configuration. Furthermore, it appears to not be consistent with the authors suggestion that the local Nusselt number should be increasing as one moves near the upper edge of the pool.

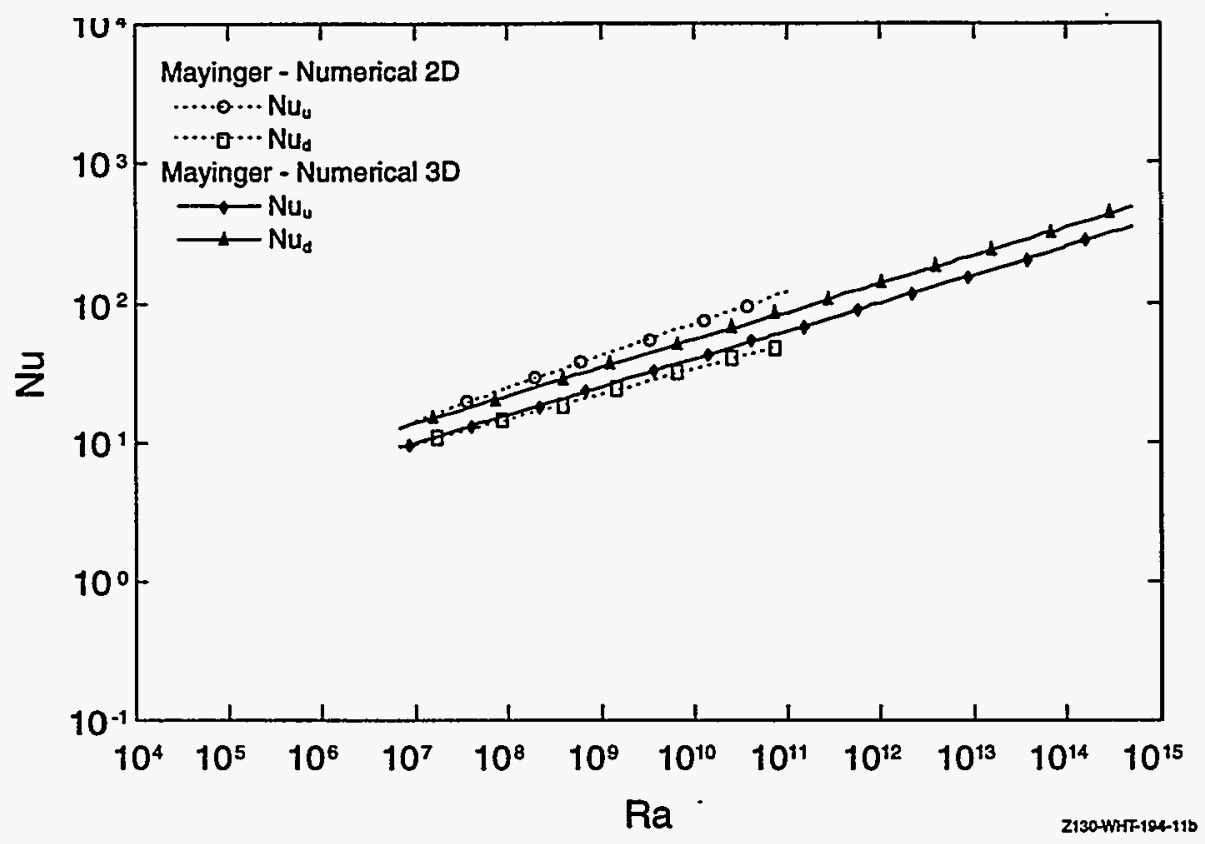

Figure A-11. Graphical comparison of numerical correlations for curved geometries.

It should be noted that in obtaining the numerical correlations with THEKAR H2, the calculations assume that the turbulent exchange coefficient are in the form of

$\varepsilon=\mathrm{a}^{\prime} \mathrm{Ra}^{\mathrm{m}^{\prime}}$

where $a^{\prime}$ and $\mathrm{m}^{\prime}$ are determined by trial and error until good agreement is obtained between the numerical calculations and results obtained from the semicircular slice experiments. Therefore, it appears that the numerically obtained correlations obtained by these authors may not be independent from the experimental data obtained.

Furthermore, Reference A-5 questions the rigorousness used to obtain THEKAR H2 results with a nonuniform mesh. It is concluded in Reference A-5 that the uniform mesh size selected is probably adequate for results obtained in the laminar regime. In the lower Rayleigh number regime, pure laminar convection occurs, thus a uniform grid distribution appears adequate because computational results compared well with experimental results. However, in the higher Rayleigh number where turbulent flow and boundary layers occur near the surface, a non-uniform mesh was used. Although results obtained with the nonuniform grid size distribution appears to agree with experimentally-obtained results, Reference A-5 suggests that a fundamentally different approach must be used if results are to be extrapolated to pools with Rayleigh numbers of interest to severe accident analysis $\left(>10^{12}\right)$. 


\section{A2.2.2 Min and Kulacki}

Reference A-15 presents results from an experimental study to investigate transient and steady-state heat transfer from an internally heated fluid layer bounded below by a spherical segment. Results from the steady state experiments are reported below; results from the transient experiments are reported in Section A3.1.2.

Volumetric energy sources used in these tests were produced by Joule heating with alternating current passing between the upper and lower surfaces of the convection chamber. The test fluid, an aqueous copper sulfate solution, was bounded from above by a rigid, isothermal horizontal surface and from below by a rigid, zero heat-flux surface. The convection chamber consisted of a right circular cylinder mounted on a spherical segment with a horizontal circular top plate which could be raised or lowered according to the depth of the fluid in the chamber. The right circular cylinder had a $0.914 \mathrm{~m}$ inner diameter and was $0.610 \mathrm{~m}$ in height. Its interior surface was covered by a $0.2 \mathrm{~mm}$ thick mylar sheet to electrically isolate it from the lower surface and the fluid. A rubber gasket was placed a the joint between the cylinder and the spherical segment to further isolate them electrically and to prevent leakage of the fluid. The spherical segment had a total polar angle of 60 degrees. Experimental data were obtained for layer geometries that range from nearly a spherical segment to a spherical segment with a superimposed horizontal layer. Two sizes of aluminum top plates were used in these experiments: a larger plate for tests with aspect ratios (the ratio of the maximum fluid depth, $\mathrm{H}$, to the spherical segment diameter, $D$ ) between 0.148 and 0.703 , and a smaller top plate for tests with an aspect ratio of 0.108 . Channels were machined into the plates for circulation of cooling water to maintain them at constant temperature. The convection chamber was thermally insulated by a minimum of $0.051 \mathrm{~m}$ of Styrofoam on all sides and a $0.006 \mathrm{~m}$ layer of plywood.

Data from the steady-state tests indicate that Nusselt numbers were influenced by the layer aspect ratio. For tests with an aspect ratio of 0.108 , which corresponds to a total polar angle of $29^{\circ}$ being encompassed, the following correlation was suggested

$\mathrm{Nu}_{\mathrm{u}}=2.086 \mathrm{Ra}^{0.127}$

based on data from tests using fluids with Prandtl numbers between 2.1 and 5.9 and Rayleigh numbers between $9.1 \times 10^{7}$ and $1.3 \times 10^{10}$.

The remaining tests considered fluids with higher aspect ratios, so that an entire total polar angle of 60 degrees was encompassed. The following correlation was developed for tests with aspect ratios of 0.148 and 0.158 , Rayleigh numbers between $3.1 \times 10^{8}$ and $3.5 \times 10^{11}$, and Prandtl numbers between 2.7 and 7.0

$\mathrm{Nu}_{\mathrm{u}}=2.320 \mathrm{Ra}^{0.125}$

For tests with an aspect ratio of 0.403 , the following correlation was obtained for fluids with Rayleigh numbers between $3.5 \times 10^{10}$ and $1.4 \times 10^{13}$ and Prandtl numbers between 2.3 and 6.8 . 
$\mathrm{Nu}_{\mathrm{u}}=1.091 \mathrm{Ra}^{0.166}$

In tests with aspect ratios between 0.686 and 0.703 , the following correlation was obtained for fluids with Rayleigh numbers between $5.5 \times 10^{11}$ and $1.6 \times 10^{14}$ and Prandtl numbers between 1.9 and 5.8.

$\mathrm{Nu}_{\mathrm{u}}=0.280 \mathrm{Ra}^{0.227}$

These correlations are graphically compared in Reference A-15. Results indicate that the correlations approach the correlation suggested by Kulacki and Emara ${ }^{\mathrm{A}-13}$ for a horizontal layer with an insulated lower boundary as the test aspect ratio increases.

In Reference $\mathrm{A}-15$, the authors indicate that the experimental uncertainty in their results for the Nusselt number is between 4.5 and $6 \%$ and for the Rayleigh number is between 24.5 and $30 \%$. This estimate represents the combined uncertainty in the thermophysical properties of the copper sulfate solution, geometrical measurements (such as the layer depth and the convection chamber dimensions), the power losses, non-uniformity in the volumetric energy source strength, and temperature measurements.

\section{A2.2.3 Kymäläinen, Tuomisto, Hongisto, and Theofanous}

Reference A-16 presents results from COPO experiments conducted to investigate heat transfer from an internally heated $\mathrm{ZnSO}_{4}-\mathrm{H}_{2} \mathrm{O}$ solution to the walls of a two-dimensional "slice" representative of the torispherical Loviisa VVER-440 reactor vessel. The test section is half-scale and geometrically similar to the Loviisa vessel lower head. It measures a span of $1.77 \mathrm{~m}$ and allows a maximum pool depth of $0.8 \mathrm{~m}$. The thickness of the slice is $0.1 \mathrm{~m}$. The flats of the test section are built from poly-carbonate plates and are heavily insulated. The inside of the plates are lined with seven pairs of electrode strips. The sides, top, and bottom walls of the facility are maintained at constant temperatures by a total of 59 separate cooling units.

Two pool heights were investigated, $0.6 \mathrm{~m}$ and $0.8 \mathrm{~m}$. The experimentally measured local heat fluxes were grouped and averaged over the pool top boundary, the vertical pool boundary, and the lower, curved pool boundary. Results indicate that the data for predicting heat transfer from the upper and side wall surfaces were in good agreement with the following correlations proposed by Steinberner and Reineke ${ }^{A-11}$ for heat transfer from a rectangular cavity (see Section A2.1.2)

$$
\begin{aligned}
& N u_{u}=0.345 \mathrm{Ra}^{0.233} \\
& N u_{s}=0.85 \mathrm{Ra}^{0.19} .
\end{aligned}
$$

It should be noted that larger variations between the data and the correlation for predicting upward heat transfer were observed for Rayleigh numbers above $5 \times 10^{14}$. For for a Rayleigh number of $1.6 \times 10^{15}$, COPO data were approximately $30 \%$ higher than predicted by Equation (A-48). 
For the downwards heat flux, the data were found to compare fairly well with the following correlation developed by Mayinger ${ }^{A-9}$ (see Section A2.2.1)

$$
\mathrm{Nu}_{\mathrm{d}}=0.54 \mathrm{Ra}^{0.18}\left(\frac{\mathrm{H}}{\mathrm{R}}\right)^{0.26}
$$

where the characteristic length used in the Nusselt and Rayleigh numbers is the curved height of the test vessel, $\mathrm{H}_{\mathrm{c}}$.

Local heat flux measurements indicate that the horizontal heat fluxes were fairly uniform. Although this result is in contrast to the results obtained by Steinberner and Reineke, ${ }^{A-11}$ the authors of Reference A-16 attribute the difference to the fact that the Rayleigh numbers investigated in the COPO facility were nearly two orders of magnitude higher and that the aspect ratios investigated in the COPO facility were considerably higher (between 3.4 and 5.6 for the COPO tests as opposed to 1 in the Steinberner and Reineke tests). Both of these factors were felt to produce unsteady and "broken" recirculation flow patterns favoring uniformity of heat flux on the vertical boundary.

Local heat flux measurements for the lower, curved boundary indicate that the decrease in heat flux is nearly linear, decreasing from approximately 2.5 to nearly zero at the bottom. This decrease is much more gradual than observed by Frantz and Dhir for the hemispherical tests reported in Reference A-18 (see Section A2.2.5) and by Asfia and Dhir for the hemispherical tests reported in Reference A-19 (see Section A2.2.6). The authors of Reference A-16 note that this difference may be due to the limitations of their "slice" geometry, which may preclude flow convergence. Additional discussion about the variation in local heat flux along the bottom surface of various test facilities may be found in Section A2.3.

\section{A2.2.4 Gabor, Cassulo, and Ellison}

Reference A-17 reports results from experiments conducted to investigate heat transfer from an internally heated $\mathrm{ZnSO}_{4}-\mathrm{H}_{2} \mathrm{O}$ pool to the walls of copper hemispherical containers, ranging from 0.24 to $0.32 \mathrm{~m}$. The copper container served both as a heat transfer surface and as an electrode. The opposing electrode was a copper disk, located at the center of the top surface of the pool. The container was cooled by water flowing through copper cooling coils, that were soldered to the outside of the hemisphere. The upper surface of the pool was open to the atmosphere. Average Nusselt number data were obtained for Rayleigh numbers ranging from $1.9 \times 10^{10}$ to 3.8 to $10^{11}$. Three different sizes of hemispherical containers were used with diameters of $0.24 \mathrm{~m}, 0.28 \mathrm{~m}$, and $0.32 \mathrm{~m}$. Heat transfer measurements were made with all three containers filled to pool depths equal to the radius. Pool depths to radius ratios of $0.5,0.667,0.813$, and 1.0 were investigated in the 0.28 diameter container.

The heat transfer to the hemispherical surface was correlated by

$$
\mathrm{Nu}_{\mathrm{d}}=0.55 \mathrm{Ra}^{0.15}
$$

The data obtained with the 0.28 diameter container with varying pool depths were correlated by 
$\mathrm{Nu}_{\mathrm{d}}=0.55 \mathrm{Ra}^{0.15}\left(\frac{\mathrm{H}}{\mathrm{R}}\right)^{1.1}$

As illustrated in Figure A-11, the data lie below the curve obtained from Mayinger et al. ${ }^{A-9}$ The authors attribute the lower values obtained from this experiment to several differences between the manner in which the experiments were conducted and in the assumptions used to derive the Mayinger experimental model. For example, the Mayinger model is based on uniform heat generation throughout the pool whereas the Gabor, et al., experiment was conducted using a heat source which decreases radially from the center electrode.

\section{A2.2.5 Frantz and Dhir}

Experiments were conducted to examine natural convection heat transfer in an internally heated partially filled spherical pool with external cooling. ${ }^{A-18}$ In these experiments, Freon-113 was contained in a Pyrex bell jar, which was cooled externally with subcooled water. The upper surface of the pool was exposed to the atmosphere. The pool was heated using a $750 \mathrm{~W}$ magnetron taken from a conventional microwave. The experiments were performed varying the pool depth and the Rayleigh number. Average Nusselt number data were obtained for Rayleigh numbers from $10^{12}$ to $10^{13}$, and pool depth to radius ratios of $0.4,0.61$, and 1.0 were studied.

Data obtained from these experiments indicate that the pool was significantly stratified, with more stratification occurring near the base of the pool than in the upper part of the pool. The local heat transfer coefficients were found to be lowest at the base of the vessel (at 0 degrees) and increase to nearly twice the mean value at pool free surface (67 to 90 degrees, depending on the value of the pool depth to radius investigated). Additional discussion about the variation in local heat flux along the bottom surface of various test facilities may be found in Section A2.3.

The mean Nusselt numbers estimated from these experiments were found to compare favorably with estimates obtained from Mayinger, et al. ${ }^{\mathrm{A}-9}$ for heat transfer along the downward, curved surface of a hemispherical cavity. Unlike results from Gabor, et al, ${ }^{\mathrm{A}-17}$ data from these experiments did not indicate that the value of the pool depth to radius ratio affected the Nusselt number.

\section{A2.2.6 Asfia and Dhir}

Additional experiments were conducted to investigate the influence of pool surface boundary conditions on natural convection heat transfer to the bounding walls of partially filled spherical cavities. ${ }^{A-19}$ Three types of boundary conditions were investigated: a free surface, a nearly insulated rigid wall, and a cooled rigid wall. These tests were performed using the same test setup used by Frantz and Dhir (see Section A2.2.6). Namely, the pools containing Freon-113 were volumetrically heated using a 750 Watt magnetron from a conventional microwave. The Freon-113 was contained in a Pyrex bell jar, that was cooled externally with subcooled water. Vessel diameters of $0.152,0.21$ and 0.44 were tested. Pool heights were varied to obtain data for pools with height to effective radius ratios between 0.21 and 1.0. Nusselt numbers were obtained for pools with Rayleigh numbers between $10^{11}$ and $10^{14}$. 
For the downward heat transfer, the following correlation was developed for predicting the dependence of Nusselt number on pool Rayleigh number

$\mathrm{Nu}_{\mathrm{d}}=0.54 \mathrm{Ra}^{0.2}\left(\frac{\mathrm{H}}{\mathrm{R}_{e}}\right)^{0.25}$

where the characteristic length used in the Nusselt and Rayleigh numbers is the pool effective radius. Heat transfer data from their tests were found to correlate with Equation (A-53) within $\pm 10 \%$. Average heat transfer coefficients from pools with rigid insulated surfaces and rigid cooled surfaces were found to be about 5-10\% higher than from pools with free surfaces. As discussed in Reference A-19, the correlation developed from this data is very similar to the numerical correlation developed by Mayinger for a three-dimensional hemispherical cavity (see Equation (A-41) and Figure A-8). Hence, it was concluded by Reference A-19 authors that the Mayinger correlation is also applicable to their data.

Figure A-12 compares ratios of the local to average heat transfer coefficient as a function of angular position measured from the lower stagnation point. Data are plotted for each type of pool surface boundary condition considered for cases where $H / R_{e}=1$. In addition, data are shown for a pool with a free upper surface and $H / R_{e}=0.4$.

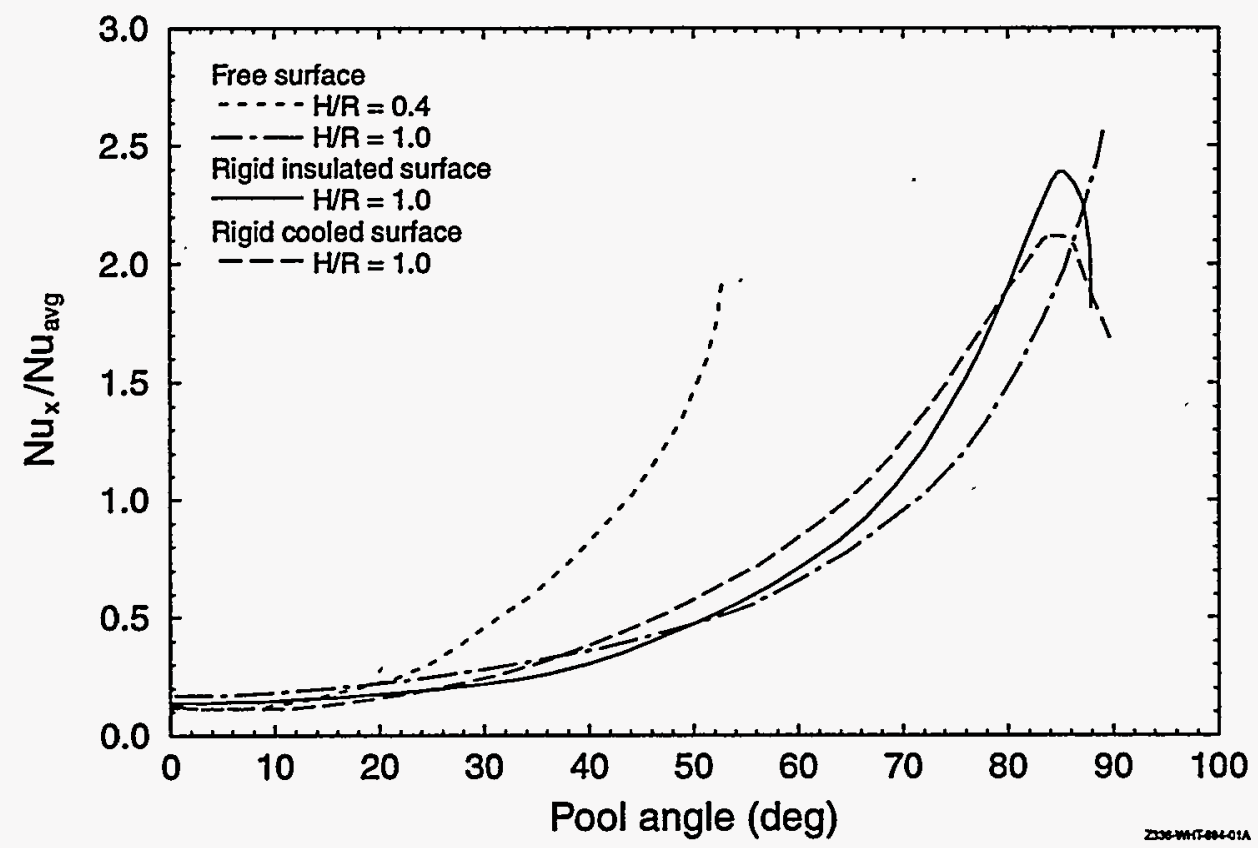

Figure A-12. Comparison of local heat transfer results from selected tests

As illustrated in Figure A-12, the heat transfer coefficient is lowest at the stagnation point and increase along the periphery of the spherical segment. This increase was observed to occur within a shorter angle in cases with $H / R_{e}<1$. In cases where a rigid insulated or cooled surface is present, the location of 
the maximum heat flux is shifted downward. Additional discussion about the variation in local heat flux along the bottom surface of various test facilities may be found in Section A2.3.

\section{A2.3 Summary and Recommendations}

As discussed in Section A1.3, the current model for predicting heat transfer from a molten pool in SCDAP/RELAP5 is based on steady-state heat transfer data obtained by Mayinger, et. al., in a two-dimensional "slice" hemispherical cavity. In the code, the total volume of the molten pool is mapped onto a hemispherical geometry, and two correlations are used to predict the average heat transfer on the upper and lower surface of this hemisphere. On the lower surface, data from Jahn and Reineke from the same hemispherical slice cavity is used to predict heat transfer as a function of angle. In this section, the applicability of correlations obtained from this test facility is reviewed in light of other data obtained for predicting heat transfer from a pool with volumetric heating at steady-state. Based on this review, several modifications to the current SCDAP/RELAP5 model for predicting heat transfer are recommended.

As discussed within Section A2.1 and Section A2.2, a considerable number of numerical and experimental investigations have been performed to predict steady-state heat transfer from molten pools. Both flat and curved geometries have been considered for two- and three-dimensional test cavities. In addition, various "fixed" boundary conditions, such as adiabatic and constant temperature conditions have been investigated. The studies are limited in the fluid Prandtl number and Rayleigh number ranges that have been considered. For example, experimental investigations typically use conducting aqueous solutions with a Prandtl number of approximately 7, which is considerably higher than the 0.03 to 0.8 range estimated for fluids in severe accident molten pools in Section A1.2; and Rayleigh numbers in the experimental investigations reviewed are less than $10^{15}$, which is considerably less than the $10^{17}$ upper limit estimated for severe accident molten pools in Section A1.2. However, there are considerably more data available for predicting heat transfer from a molten pool than were available at the time that the current model was implemented into SCDAP/RELAP5.

Figure A-13 through Figure A-15 compare correlations obtained from investigations concentrating on fluids with higher Rayleigh numbers. Figure A-13 compares correlations for predicting upward heat transfer; Figure A-14 compares correlations for predicting horizontal heat transfer; and Figure A-15 compares correlations for predicting downward heat transfer. In these figures, curves marked "Mayinger -experimental slice" correspond to correlations currently used in SCDAP/RELAP5. A dashed curve, labeled "Recommended," is included in each figure that corresponds to correlations that this review indicates should be used for modeling molten pool behavior in SCDAP/RELAP5. These "Recommended" correlations correspond to data obtained from the Kymäläinen, et al. COPO tests. As noted in Section A2.2.3, these tests considered higher Rayleigh number fluids. Furthermore, as illustrated in Figure A-13 through Figure A-15, these correlations are consistent with many of the correlations recommended from several other two and three dimensional investigations. 


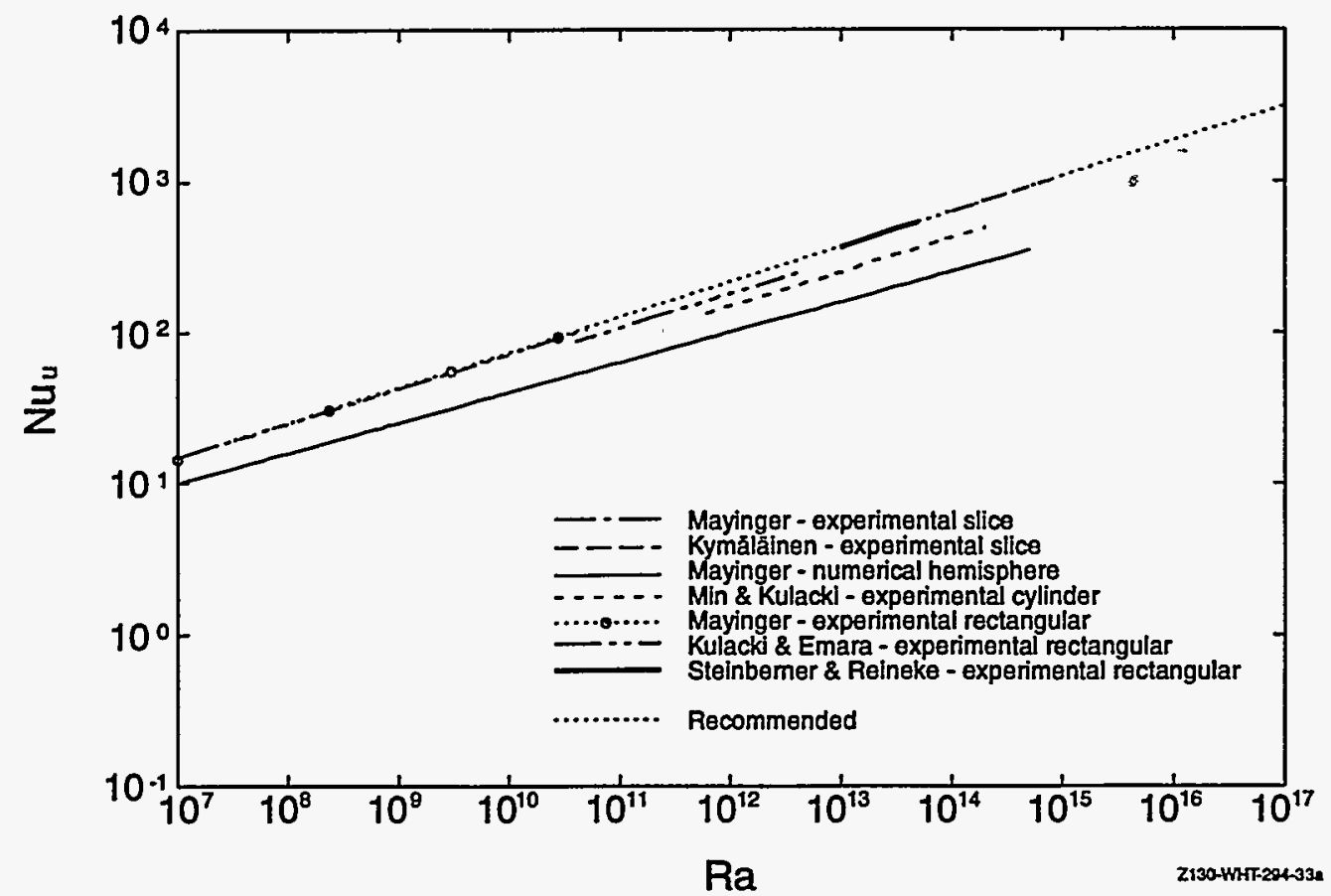

Figure A-13. Comparison of high Rayleigh number correlations for predicting upward heat transfer.

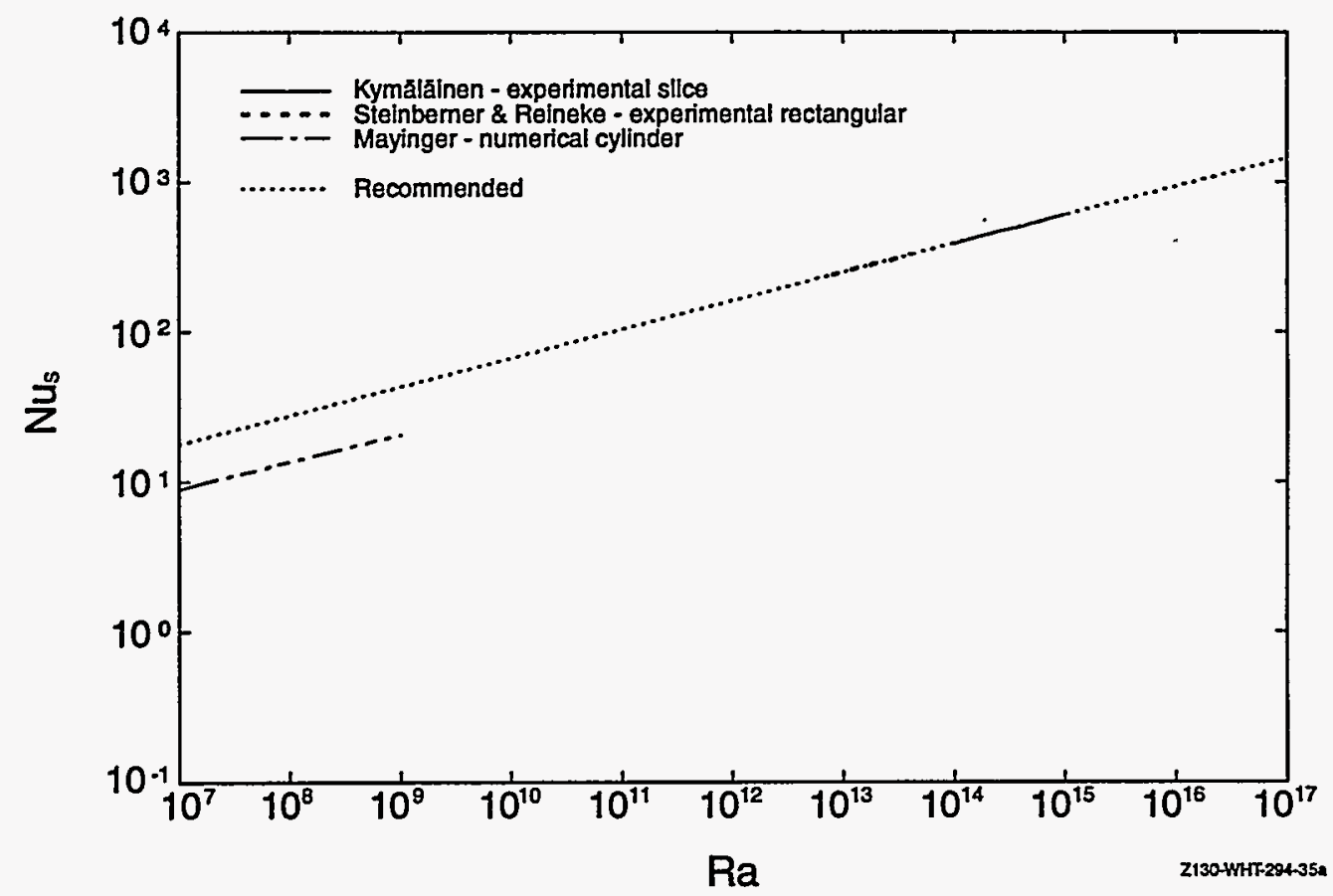

Figure A-14. Comparison of high Rayleigh number correlations for predicting horizontal heat transfer (to side walls). 


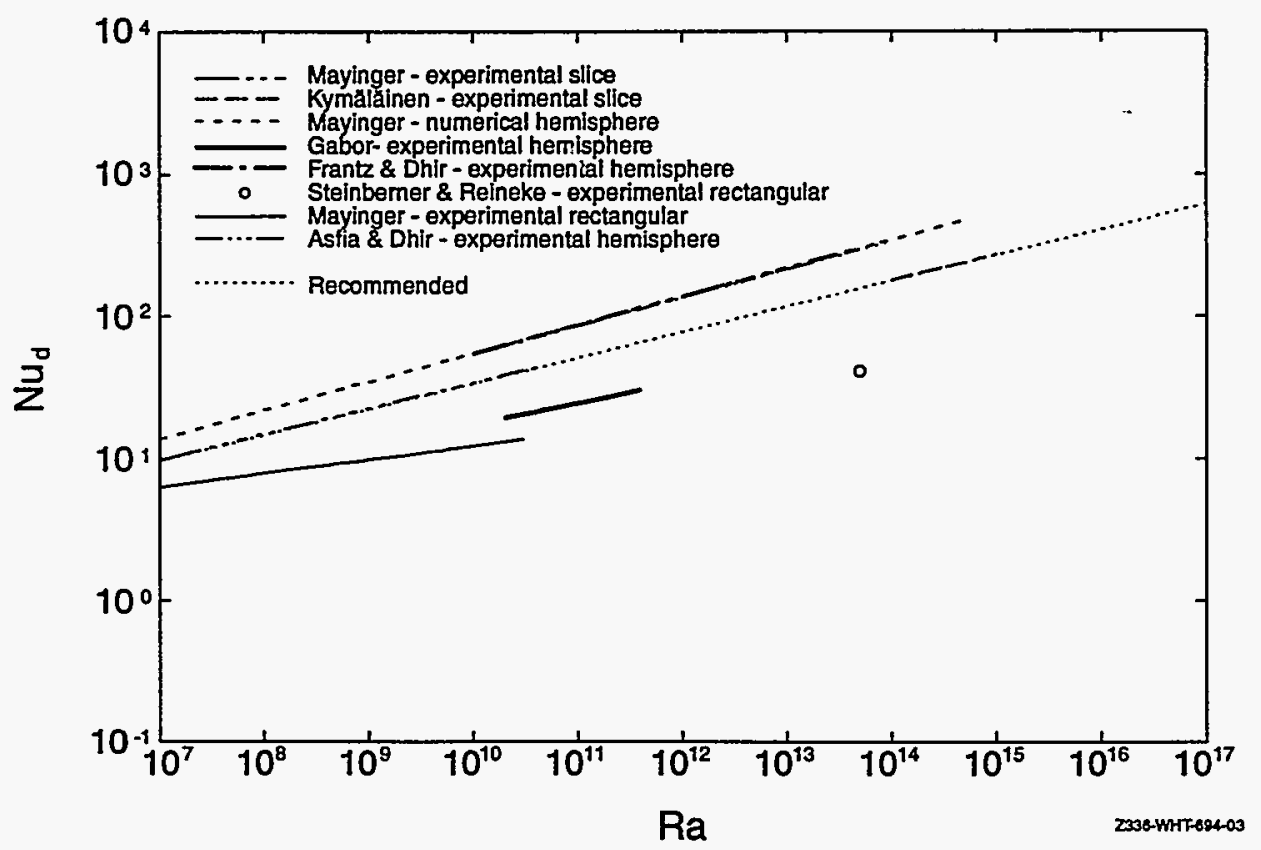

Figure A-15. Comparison of high Rayleigh number correlations for predicting downward heat transfer.

It is interesting to note that although the correlations plotted in these figures were obtained from various cavity geometries, there is generally less scatter than from correlations obtained from a single geometry. An obvious exception where more scatter is observed is with correlations obtained for hemispherical geometries (Mayinger - numerical hemisphere, Frantz and Dhir - experimental hemisphere, Asfia and Dhir - experimental hemisphere, Gabor - experimental hemisphere). Although one might conclude that these differences suggest that two-dimensional data are not applicable to three-dimensional geometries, one should note that there is considerable disagreement among the three-dimensional investigations. Furthermore, information in Section A2.2 suggests that there may have been more uncertainty associated with the experimental hemispherical investigations and that the numerical study may have incorrectly modeled turbulent heat transfer. Therefore, this study recommends the correlations corresponding to the two-dimensional COPO test results, which in many cases are consistent with the Steinberner and Reineke correlations obtained using a three-dimensional rectangular cavity. ${ }^{a}$

Figure A-16 compares local heat flux measurements for the lower, curved boundary in the Mayinger, et al. slice tests, the Frantz and Dhir hemispherical tests, the Asfia and Dhir hemispherical tests, and the Kymäläinen, et al. COPO slice tests. Although results in Figure A-16 indicate a significant difference in the manner that the local heat transfer coefficient will vary as a function of angle along the pool's lower surface, the curves are similar in that they go from nearly zero on the bottom surface (at zero degrees) to approximately a factor of 2 or 2.5 at the point where the bottom intersects the vertical wall (at approximately 90 degrees for the Frantz and Dhir, the Asfia and Dhir $H / R_{e}=1$ tests, and the Mayinger

a. Discussions indicate that the recommended correlations are consistent with results from recently completed natural convection heat transfer tests at the University of California - Santa Barbara. ${ }^{\text {A-20 }}$ 
tests, approximately 50 degrees for the Asfia and Dhir $\mathrm{H} / \mathrm{R}_{e}=1$ test, and approximately 45 degrees for the Kymäläinen tests). Results from geometries with pool depth to radius ratios less than unity consistently indicate a much steeper rise in local heat transfer. However, results from the torispherical COPO slice tests indicate a much more linear increase in the local heat transfer coefficient than observed in any of the other experiments. Although data from the recently obtained Asfia and Dhir and Frantz and Dhir hemispherical tests are consistent, it should be noted that they were obtained from the same experimental setup. Results from the Asfia and Dhir tests also indicate that upper pool surface boundary conditions also have a slight effect on variations in the heat transfer coefficient.

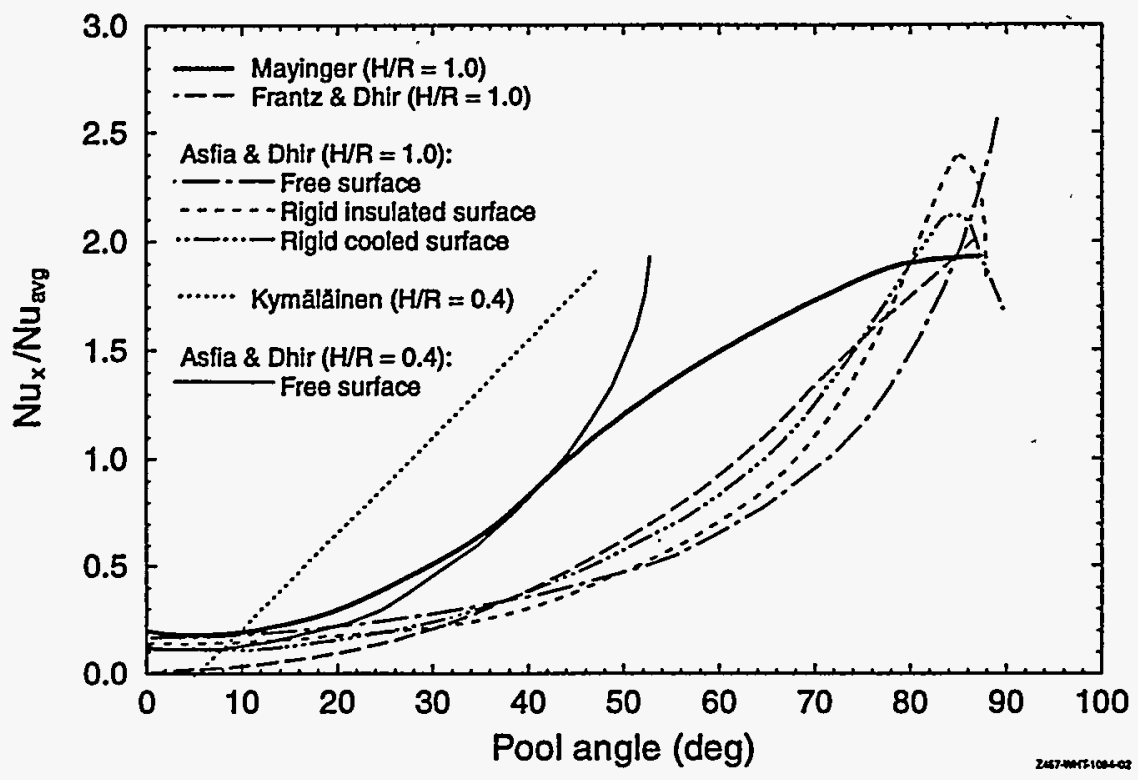

Figure A-16. Comparison of local heat transfer results

Because the data for predicting local heat transfer are limited and because the Mayinger curve, which is currently used in SCDAP/RELAP5, appears to give an average of the results obtained from other experimental setups, it is suggested that SCDAP/RELAP5 continue to use the Mayinger results for modeling variations in the local heat transfer coefficient along the bottom surface. Note that the Mayinger results are applied in a manner that allows heat transfer coefficients to rise more rapidly along pool surfaces with pool depth to radius ratios less than unity so that the maximum value of approximately 2 occurs at the point where the bottom intersects the vertical wall.

It should be noted that the proposed recommendations would result in the code's model considering semicircular segment and torispherical geometries rather than mapping the pool's shape onto a hemispherical geometry. As discussed above, this recommendation will be implemented by including a separate correlation for horizontal heat transfer and applying the geometry correction factor suggested by Kymäläinen (and originally in the Mayinger, et al correlation). Scoping calculations were performed to illustrate the effects of accommodating geometry effects using the recommended downward heat transfer correlations. In these calculations, the $80 \mathrm{wt} \% \mathrm{UO}_{2}$ and $20 \mathrm{wt} \% \mathrm{ZrO}_{2}$ mixed oxide compound predicted in the Reference A-3 calculations was assumed. As illustrated below in Figure A-17, results indicate that heat transfer coefficients do vary when the correct geometry is considered. For the example considered, these geometry effects were found to impact the heat transfer coefficient by up to $20 \%$. 


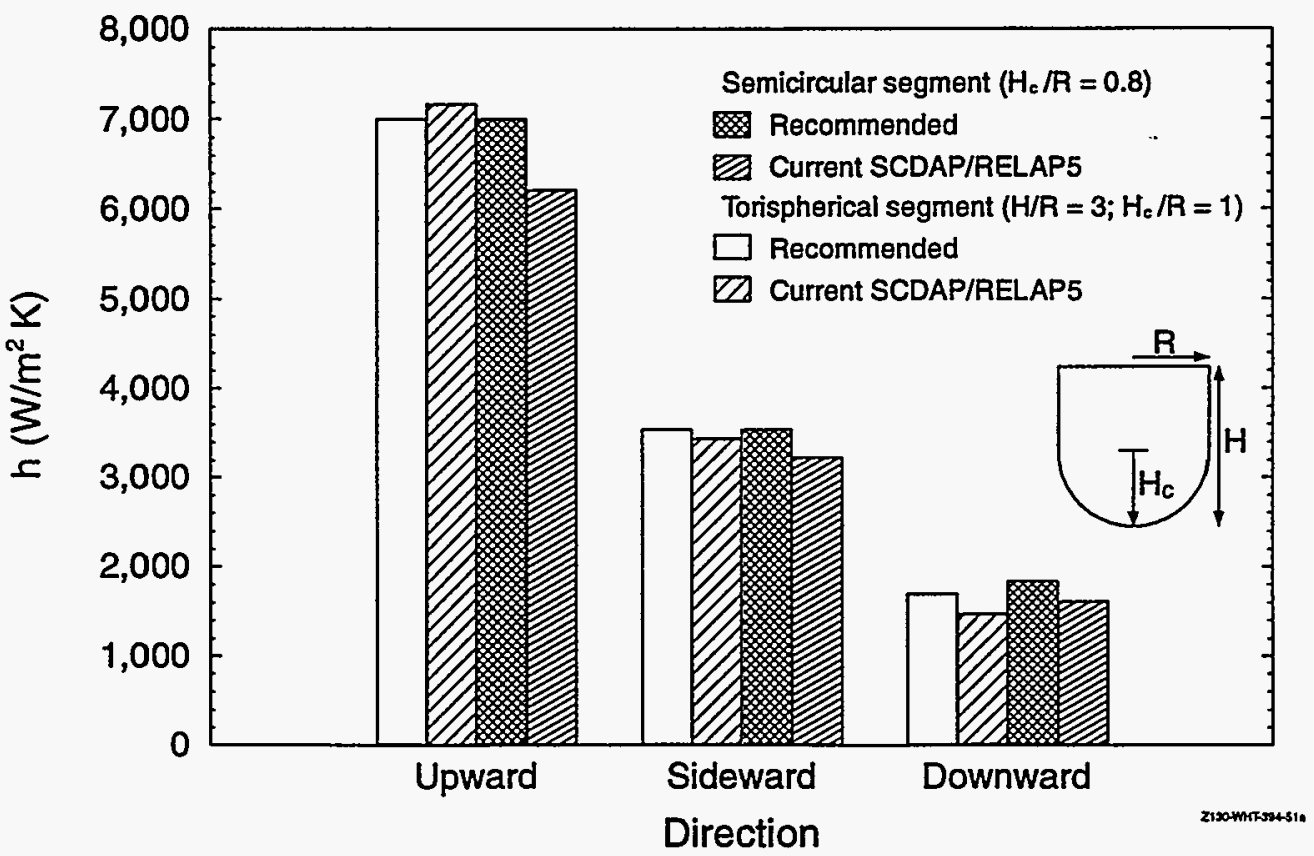

Figure A-17. Comparison of heat transfer coefficients predicted when geometry effects are considered.

In summary, it is suggested that the steady-state heat transfer correlations used in SCDAP/RELAP5 be modified to reflect data obtained from more recently completed, higher Rayleigh number results from the COPO facility. Note that implementation of this recommendation would allow more general shapes of molten pools to be modeled (from a semicircular segment to a cylinder bounded below by a curved lower surface). Data for predicting variations in local heat transfer on the curved lower surface were also reviewed. However, at this time it is felt that SCDAP/RELAP5 should continue to use the Mayinger data for predicting local values on the lower surface.

\section{A3. TRANSIENT INVESTIGATIONS}

For the high Rayleigh numbers that will occur in large pools during a severe accident, experimental evidence discussed in Section A2 indicates that the thermal resistance in convecting regions is determined by relatively thin boundary layers. Thus, several references [A-6, A-35, and A-36] suggest that it is more appropriate to model heat transfer from such pools using correlations based on the temperatures differences that exist across the convecting region because this approach allows lateral cooling to be included and transient calculations to be performed. Unfortunately, only a limited number of experimental investigations reporting transient natural convection data from pools with volumetric heating sources were found in the literature. However, these data can be used to estimate the time periods required for steady-state natural convection to become established in a molten pool and to illustrate that significant error may be introduced by applying a steady-state correlation to a pool prior to the time that steady-state convection is established. In addition, analytical techniques were used to predict heat transfer from a transient molten pool using data obtained from steady-state experiments. Transient pool data from one of the references investigating transient and steady-state heat transfer are used in Section A3.2 to validate an 
analytical method for predicting transient heat transfer from the pool using results from steady-state experiments.

\section{A3.1 Experimental Data}

In this review of the literature, only two references were found which investigated transient heat transfer from a molten pool. These references reported correlations relating the pool Fourier number and the step increase or decrease in pool Rayleigh number. The references also report the temperature differences occurring in the pool during the transient time periods. Section A3.1.1 and Section A3.1.2 report the correlations obtained between the pool Fourier number and the step changes in pool Rayleigh number. These correlations are applied in Section A3.1.3 to estimate time periods required before steady-state heat transfer can be established in representative molten pools that may form during a severe accident.

\section{A3.1.1 Kulacki and Emara}

In Reference A-13, Kulacki and Emara document results from an experimental investigation of steady and transient thermal convection in a rectangular cavity containing aqueous silver nitrate solution with volumetric heat sources. The experimental setup and results from the steady-state experiments are discussed in Section A2.1.5; results from the transient experiments are discussed below.

Transient convection was investigated by performing tests in which the Rayleigh number was suddenly changed from zero to a value in the laminar regime, then to a value in the transition regime $\left(\mathrm{Ra} \approx 10^{9}\right)$, and finally to a value in the fully turbulent regime $\left(\mathrm{Ra} \geq 10^{9}\right)$. A similar series of runs was performed in which the power input to the layer was suddenly turned off after steady convection at a given Rayleigh number had been established.

Data from the transient experiments were used to obtain a relationship between the step change in Rayleigh number and the time when a thermocouple is within $2 \%$ of its steady-state value. For experiments investigating step increases in power, the Fourier number required for the maximum temperature difference to reach $2 \%$ of it steady-state value may be estimated using

Fo $=13.419(\Delta \mathrm{Ra})^{-0.213}$.

For step decreases in power, the Fourier number required to reach an isothermal steady state is given by

$F_{0}=13.877(\Delta \mathrm{Ra})^{-0.215}$

In Reference A-13, the authors indicate that the experimental uncertainty in the Rayleigh number is between 5 and $7 \%$. This estimate represents the combined uncertainty in the thermophysical properties of the aqueous silver nitrate solutions, geometrical factors (primarily the depth of the layer), and the power consumption per unit volume. In the transient experiments, temperature measurements were estimated to be within 5 to $10 \%$. This uncertainty represents the combined uncertainty in measurements taken from the 
strip chart and the accuracy of the thermocouple probes. The uncertainty in the layer Fourier number values was estimated as 2 to $3 \%$.

\section{A3.1.2 Min and Kulacki}

Reference A-15 presents results from an experimental study to investigate transient and steady-state heat transfer from an internally heated fluid layer bounded below by a spherical segment. The experimental setup and results from the steady-state experiments are discussed in Section A2.2.2. Results from the transient experiments are discussed below.

Transient convection was investigated by obtaining data from tests in which step changes in the Rayleigh number were made in order to determine the time scales required for convection to develop and decay. Steady-state Nusselt numbers and maximum temperature differences between the upper and lower boundaries obtained from the transient and steady-stated experiments were compared and found to be in good agreement. Data from the transient experiments in which volumetric heating was suddenly started indicate that the Fourier number can be correlated to the step increase in Rayleigh number using the following relationships

$F_{0}=23.34(\Delta \mathrm{Ra})^{-0.223}$ for $\mathrm{z} / \mathrm{L}=0$

$F_{0}=58.06(\Delta \mathrm{Ra})^{-0.266}$ for $\mathrm{z} / \mathrm{L}=0.5$

where $\mathrm{z}$ represents the height of fluid from a base of length, $\mathrm{L}$ (thus, longer Fourier numbers and time periods are predicted for equations with $\mathrm{z} / \mathrm{L}=0$ ). For tests where steady-state convection was established and volumetric heating was suddenly stopped, the following relationships were obtained

$\mathrm{Fo}=23.58(\Delta \mathrm{Ra})^{-0.224}$ for $\mathrm{z} / \mathrm{L}=0$

$F_{0}=57.45(\Delta \mathrm{Ra})^{-0.267}$ for $\mathrm{z} / \mathrm{L}=0.5$

All the above relationships (for both increases and decreases in volumetric heating) were obtained for fluids with Rayleigh numbers between $1.3 \times 10^{8}$ and $3.0 \times 10^{13}$ and pools with aspect ratios between 0.108 and 0.681 .

In Reference $\mathrm{A}-15$, the authors indicate that the experimental uncertainty in their results for the Rayleigh number is between 24.5 and $30 \%$. This estimate represents the combined uncertainty in the thermophysical properties of the copper sulfate solution, geometrical measurements (such as the layer depth and the convection chamber dimensions), the power losses, non-uniformity in the volumetric energy source strength, and temperature measurements. For the transient experiments, the uncertainty for the Fourier number is estimated to be between 5.5 and $8 \%$. This estimate represents the combined uncertainty due to time measurement, geometrical measurements, and thermophysical properties of the copper sulfate solution. 
Equation (A-56) is compared in Figure A-18 with Equation (A-54) from Section A3.1.1, which was proposed by Kulacki and Emara for a horizontal cavity. ${ }^{A-13}$ Results shown in Figure A-18 indicate that higher Fourier numbers (and thus longer time periods required for steady-state convection) are predicted by the Kulacki and Emara correlation.

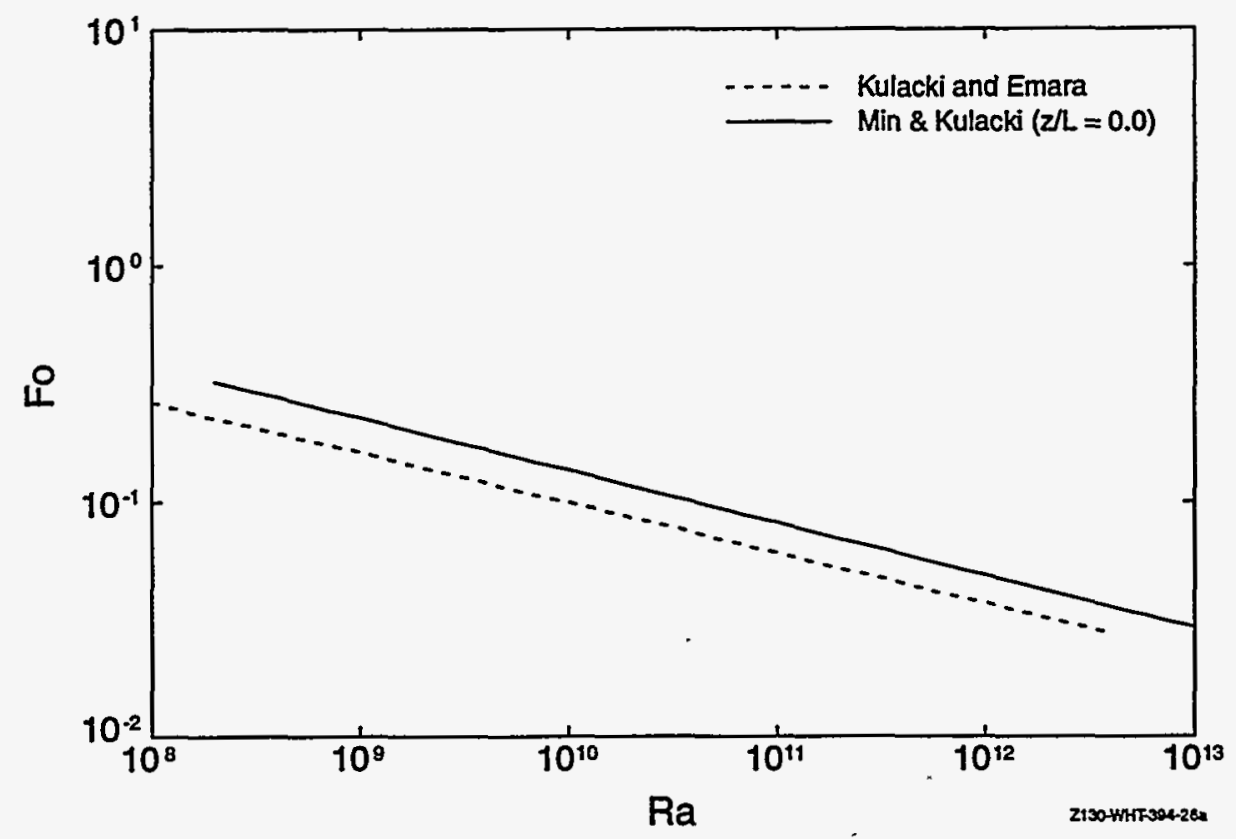

Figure A-18. Comparison of transient convection heat transfer correlations.

\section{A3.1.3 Representative Values for Transient Time Periods}

Using the correlations proposed by Kulacki and Emara and by Min and Kulacki, it is of interest to estimate the time periods required before steady-state heat transfer is established in a molten pool during a severe accident. Using the representative pool configurations and material compositions described in Section A1.2, representative transient time periods are summarized below in Table A-4. 
Table A-4. Representative time periods required for steady-state heat transfer.

\begin{tabular}{|c|c|c|}
\hline \multirow{2}{*}{$\begin{array}{c}\text { Molten Pool Rayleigh Number } \\
\text { (Lower/Upper) }\end{array}$} & $\begin{array}{c}\text { Time till Steady-State, hours } \\
\text { (Lower/Upper) }\end{array}$ \\
\cline { 2 - 3 } & $\begin{array}{c}\text { Kulacki and } \\
\text { Emara }\end{array}$ & $\begin{array}{c}\text { Min and } \\
\text { Kulacki } \\
\mathbf{z} / \mathbf{L}=\mathbf{0 . 0}\end{array}$ \\
\hline \hline \multicolumn{3}{|c|}{ Ceramic Material } \\
3e12/3e17 & $2.0 / 5.1$ & $2.3 / 6.6$ \\
\hline Metallic Material \\
3e13/2e17
\end{tabular}

One should note that for similar pool geometries and compositions, the correlations between Fourier numbers and Rayleigh numbers would yield shorter time periods for steady-state natural convection with higher Rayleigh numbers. However, values in the above table illustrate that the pool characteristic length and material properties play an important role in estimating the time periods required for steady-state convection. For example, smaller time periods are required for pools with metallic melt because of the much higher thermal diffusivity for metallic melt (see Table A-1). Likewise, the time periods estimated for the mixed oxide melt are considerably higher because of the lower thermal diffusivity for this material (see Table A-1).

As indicated in Table A-4, the time periods required before steady-state convection is established is significant for the time periods of interest in most severe accident analysis. For example, in the SCDAPI RELAP5/MOD3.1 analysis described in Section A1.2, the molten pool that forms in the core region has a steady-state Rayleigh number of 1 e16 and is only present for approximately 2000 seconds ( 0.55 hours) before the crust surrounding the pool is predicted to fail. This time period is considerably less than the 6 to 11 hour time periods estimated above for steady-state natural convection to occur in a mixed oxide pool. Therefore, steady-state natural convection is never predicted to occur in the Section A1.2 calculations. Furthermore, in cases where a molten pool could be sustained in the core or vessel region for the required 6 to 11 hours, other effects, such as pool growth and mass addition, would likely keep the pool from achieving a steady state.

\section{A3.2 Analytical Investigations}

References A-6, A-35, and A-36 describe a method for modeling heat transfer from a molten pool during the transient and steady-state stages by converting empirical correlations based on a steady-state Rayleigh number to correlations based on a transient Rayleigh number. Within this study, this method is validated using the data obtained by Min and Kulacki. Furthermore, the Min and Kulacki data are used to 
illustrate the error incurred by applying a steady-state correlation prior to the time when a pool is at steady-state.

\section{A3.2.1 Turland and Morgan}

In Reference A-6, the following method is presented for obtaining a transient correlation from a steady-state correlation. In general, steady-state correlations have the form

$\mathrm{Nu}=\mathrm{A}_{1} \mathrm{Ra}^{\gamma}$

where the steady-state Nusselt number and the steady-state Rayleigh number are given by

$N u=\frac{q^{\prime \prime} L^{2}}{k \Delta T}$

$\mathrm{Ra}=\frac{\mathrm{g} \beta \mathrm{q}^{\prime \prime} \mathrm{L}^{5}}{\alpha v \mathrm{k}}=\mathrm{Ra} \mathrm{a}^{\prime} \mathrm{Nu}$

Using a transient Rayleigh number, $\mathrm{Ra}^{\prime}$, given by

$\mathrm{Ra}^{\prime}=\frac{\mathrm{Ra}}{\mathrm{Nu}}=\frac{\mathrm{g} \beta \mathrm{L}^{3} \Delta \mathrm{T}}{\alpha \mathrm{V}}$

the correlation given by Equation (A-60) is equivalent to

$N u=\left(A_{1} R a^{\prime \gamma}\right)^{\frac{1}{1-\gamma}}$

The above method has been successfully implemented in severe accident analysis codes, such as the PAMPUR $^{\mathrm{A}-38}$ and MELTPV ${ }^{\mathrm{A}-35}$ codes.

In References A-6 and A-35, it is noted that the above method has been validated using data obtained by Kulacki and Emara for a horizontal layer. In Section A3.2.2 of this study, the above method is validated using the data obtained by Min and Kulacki for a cylinder bounded below by a spherical segment (Reference A-15). In addition, the Min and Kulacki data are used to illustrate the error introduced when a steady-state correlation is applied to a transient pool. 


\section{A3.2.2 Application of Transient Method to Experimental Data}

Min and Kulacki give data from steady-state and transient three-dimensional experiments in Reference A-15. The approach described above for obtaining a heat transfer correlation applicable to a transient pool was applied to the data in Reference A-15. Because the material properties used by the Min and Kulacki data to obtain the correlations presented in Reference A-15 were not available, it was necessary to recorrelate the data assuming the temperature-dependent properties for pure water at atmospheric pressure given in Reference A-37. Using the data obtained by Min and Kulacki for tests with L/D approximately equal to 0.403 , the data could be predicted to within $12 \%$ using the following steady-state correlation

$\mathrm{Nu}=0.366 \mathrm{Ra}^{0.207}$

This equation corresponds to the following transient Rayleigh number correlation

$\mathrm{Nu}=0.281 \mathrm{Ra}^{0.0262}$

Note that the definition of the transient Rayleigh number, $\mathrm{Ra}^{\prime}$, is slightly different than the definition given by Equation (A-5) because of the geometry used in the Min and Kulacki tests. For the Min and Kulacki tests, the transient Rayleigh number simplifies to

$\mathrm{Ra}^{\prime}=\frac{\mathrm{g} \beta \Delta \mathrm{TL}^{4}}{\alpha \mathrm{V}} \frac{4 \mathrm{~V}}{\pi \mathrm{D}^{2}}$

where the volume, $\mathrm{V}$, is the sum of the spherical section and the cylindrical section used in these experiments (see Section A2.2.2).

To illustrate that the transient correlation was applicable to steady-state pool data, results obtained with a steady-state correlation and a transient correlation extracted from the steady-state correlation are compared with the experimental data in Figure A-19. As illustrated in this figure, the transient correlation predicts the steady-state experimental data nearly as well as the steady-state correlation (the transient correlation is within $15 \%$ of the experimental values, whereas the steady-state correlation is within $12 \%$ of the experimental values).

However, when the steady-state and transient correlations are applied to transient pool data, large differences are observed. Figure A-20 compares predictions from the transient and steady-state correlation as a function of transient Rayleigh number. In this figure, three transient tests are considered (Runs 149, 150 , and 151) corresponding to the three step increases in the steady-state predictions. While the transient correlation indicates a smooth increase in Nusselt number, corresponding to the temperature increases observed in the pool, the steady-state correlation predicts a constant Nusselt number corresponding to the volumetric heat generation rate in the pool. As illustrated in Figure A-20, it is not correct to apply a steady-state correlation to predict heat transfer from a transient molten pool. 


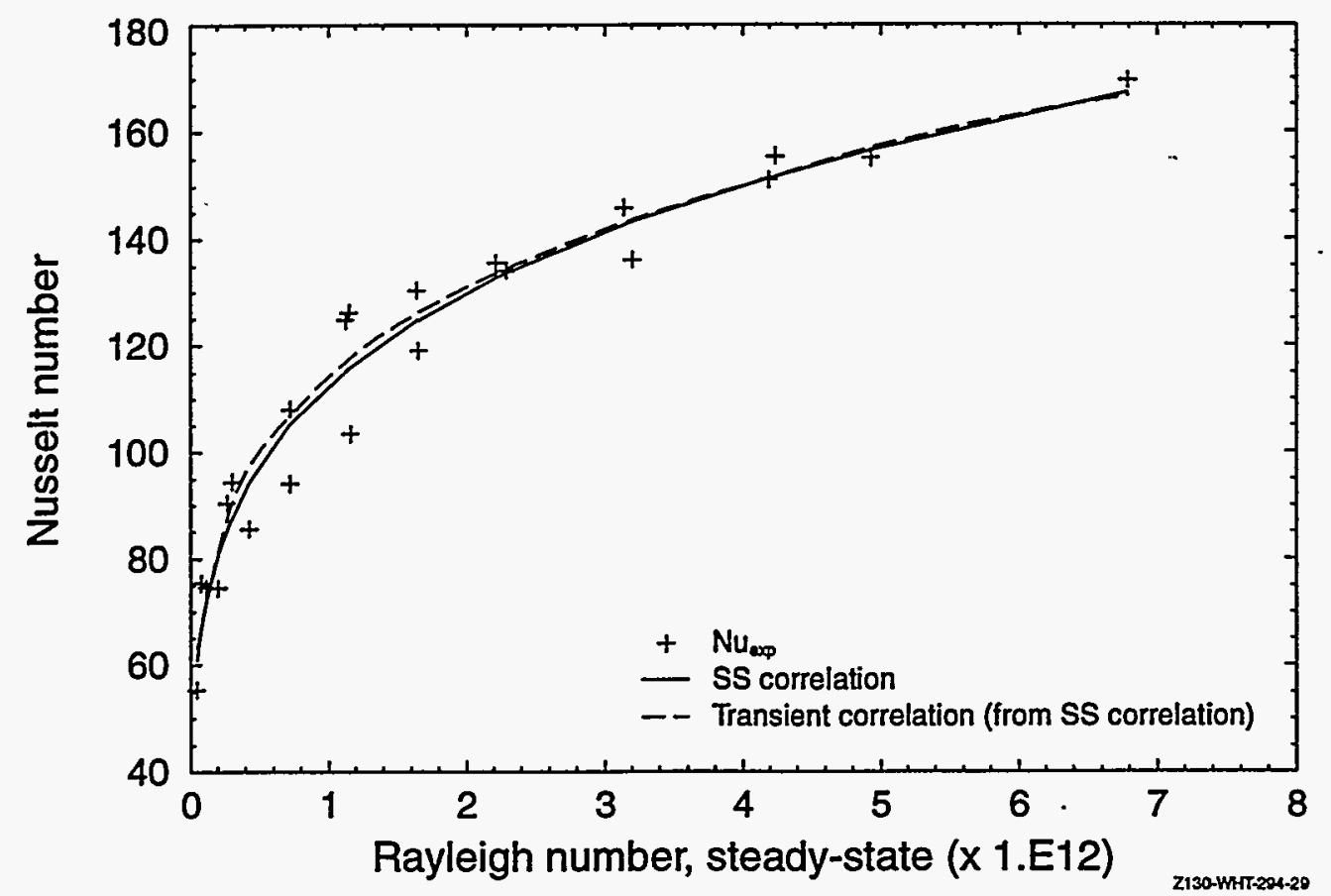

Figure A-19. Comparison of steady-state and transient correlations with steady-state experimental data.

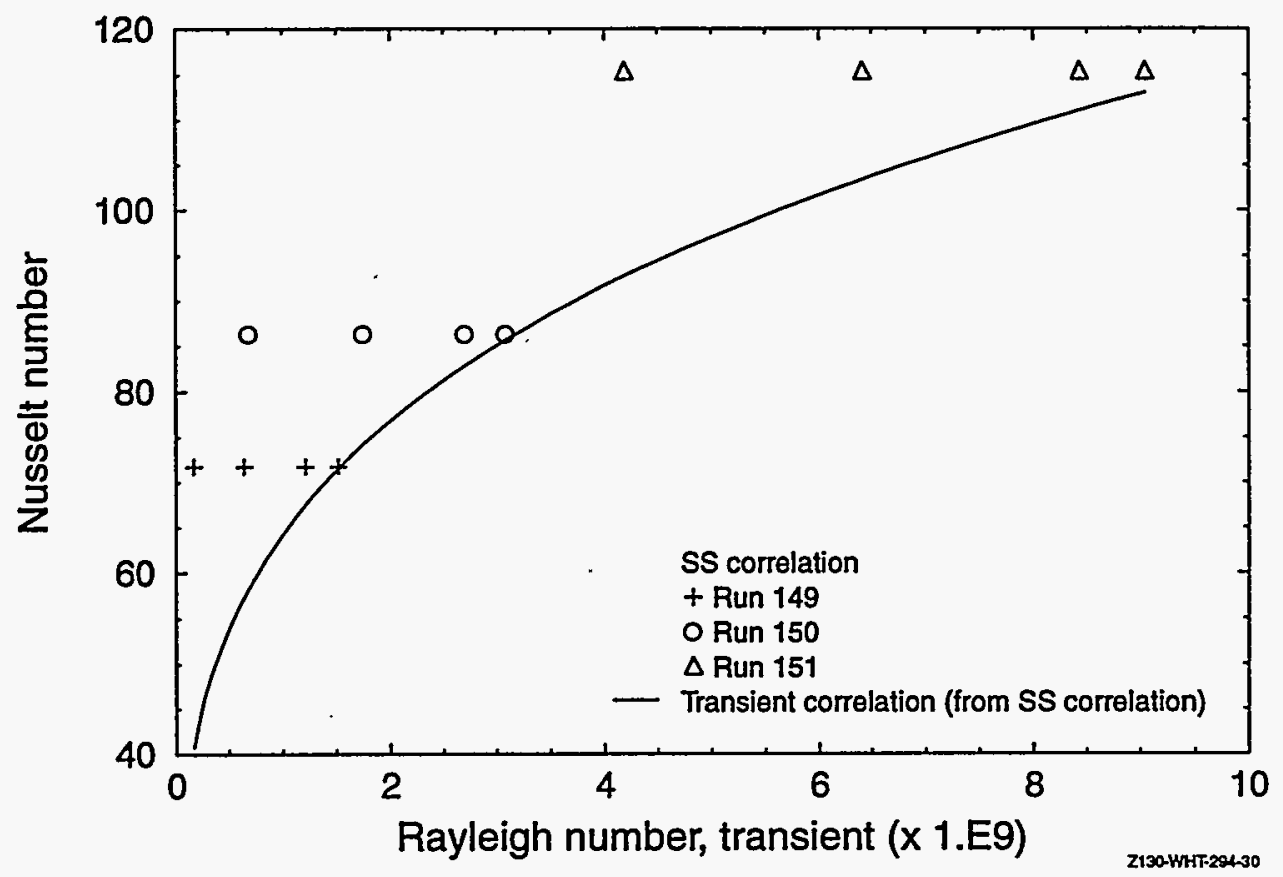

Figure A-20. Comparison of transient and steady-state correlations as a function of transient Rayleigh number. 


\section{A3.3 Summary and Recommendations}

Information presented in this section indicates that transient correlations should be obtained from the steady-state molten pool heat transfer correlations. To emphasize the magnitude by which the steady-state correlations may overpredict heat transfer if they are applied erroneously to a transient pool, calculations were performed comparing transient and steady-state heat transfer correlation predictions assuming the molten pool geometry and composition predicted in the SCDAP/RELAP5/MOD3.1 analysis described in Section A1.2. In these calculations, the following steady-state heat transfer correlation currently in SCDAP/RELAP5 for predicting downward heat transfer was assumed.

$\mathrm{Nu}_{\mathrm{d}}=0.54 \mathrm{Ra}^{0.18}$.

Using the method described in Section A3.2.1, this correlation translates to the following transient correlation

$\mathrm{Nu}_{\mathrm{d}}=0.47 \mathrm{Ra}^{0.22}$

Using parameters, such as the pool temperatures, characteristic lengths, volumetric heat generation rate and melt material composition for the SCDAP/RELAP5 analyses described in Section A1.2, transient and steady-state Nusselt numbers for the pool that forms in the core region at 17,500 seconds were estimated. Results are compared below in Figure A-21. Note that results presented in Section A3.1.3 indicate that the pool has not reached a steady-state before it breaks through its ceramic crust and relocates to the lower head. 


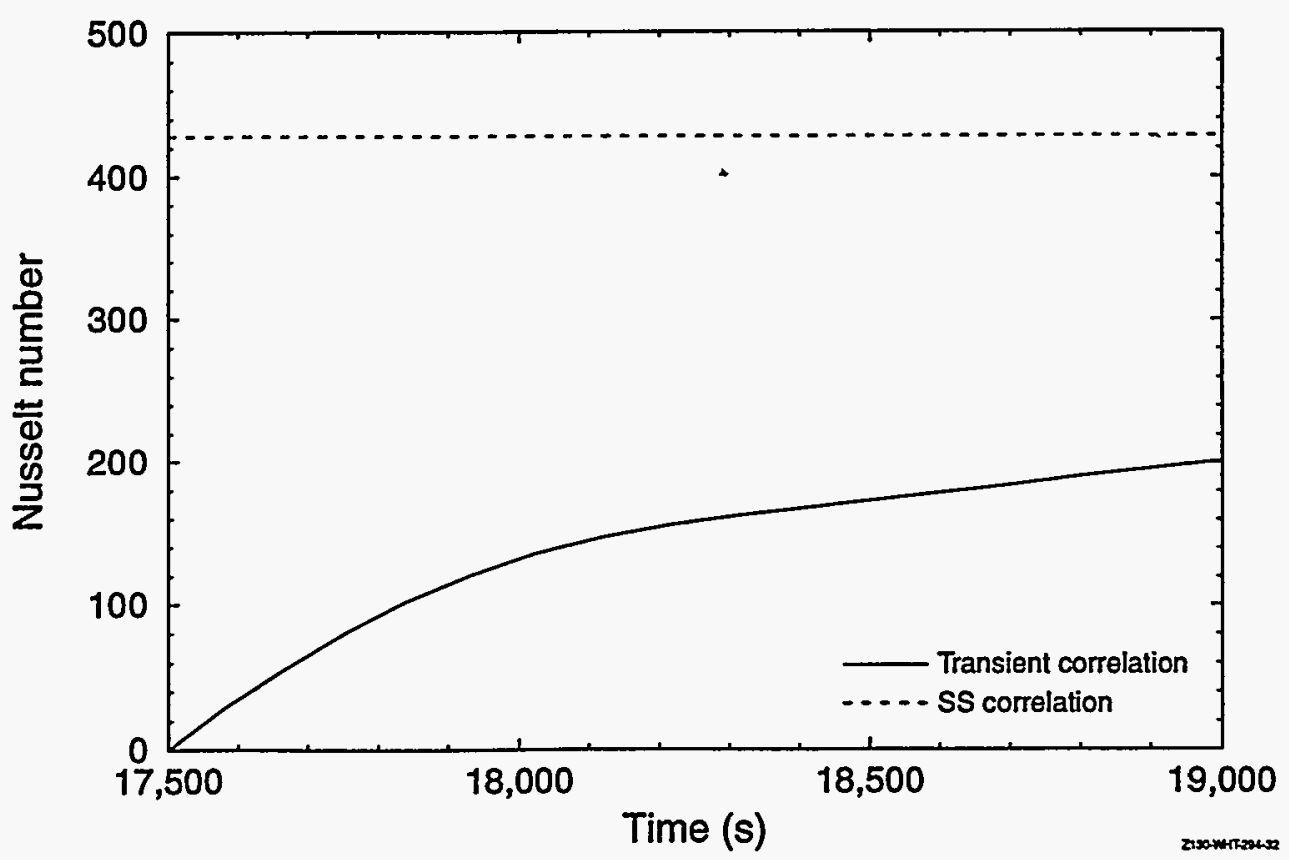

Figure A-21. Comparison of transient and steady-state Nusselt numbers predicted for the molten pool predicted in the SCDAP/RELAP5 described in Section A1.2.

As illustrated above in Figure A-21, the Nusselt number predicted with the steady-state correlation is significantly higher (at least a factor of two) than that predicted with the transient correlation. Therefore, it is strongly recommended that the SCDAP/RELAP5 molten pool heat transfer correlations be modified to transient correlations.

\section{A4. PROTOTYPIC MATERIAL INVESTIGATIONS}

As discussed in Section A1.2, the Prandtl number for molten pool material is expected to range between approximately 0.05 and 0.8 . These values are considerably lower than the values for fluids used in experiments reviewed in Section A2 and Section A3 (typically ranging around 7.0). Although some analytical studies have postulated heat transfer correlations including the effect of fluid Prandtl number, ${ }^{A-9, A-14}$ no experiments were performed to validate the relationships proposed in these references. A limited number of experiments were performed using electrically heated $\mathrm{UO}_{2}$. As discussed in this section, results from these experiments are significantly different than results obtained from lower temperature, aqueous solution experiments. However, there are significant uncertainties associated with data obtained from these tests as will be discussed below.

\section{A4.1 Experimental Data}

References A-21, A-22, and A-23 discuss experiments performed to investigate heat transfer from a pool of containing between 10 and $20 \mathrm{~kg}$ of molten $\mathrm{UO}_{2}$. The melt was contained in rectangular cavities (either 0.1 by $0.1 \mathrm{~m}$ or 0.2 by $0.2 \mathrm{~m}$ ). Volumetric heat generation rates varied from 5 to $12 \mathrm{MW} / \mathrm{m}^{3}$ ). The two test cavities used in these experiments were composed of two vertical walls and a bottom containing 
stainless steel U-shaped pieces electrically insulated from each other by layers of zirconia paper. The other parallel vertical walls were tungsten plates, which served as the electrodes for electrical current supply to the molten $\mathrm{UO}_{2}$ in the container. The top surface of each test cavity was a copper plate. All six surfaces of each test cavity were cooled. In each test, $\mathrm{UO}_{2}$ powder was placed in a cavity where it was heated by passing electrical current through a tantalum wire coil connected between the electrodes. Eventually, the wire coil would melt and a molten pool of electrically conducting $\mathrm{UO}_{2}$ forms at the bottom of the cell in electrical contact with the electrodes, but insulated from the other surfaces of the cell by unmelted powder. Figure A-22 illustrates the typical molten pool configuration inferred from post-test examinations. As indicated in this figure, the solidified mass usually had several smaller voids below a large main void. These smaller voids were believed to form as a result of non-uniform solidification and shrinkage during cool-down after the experiment.

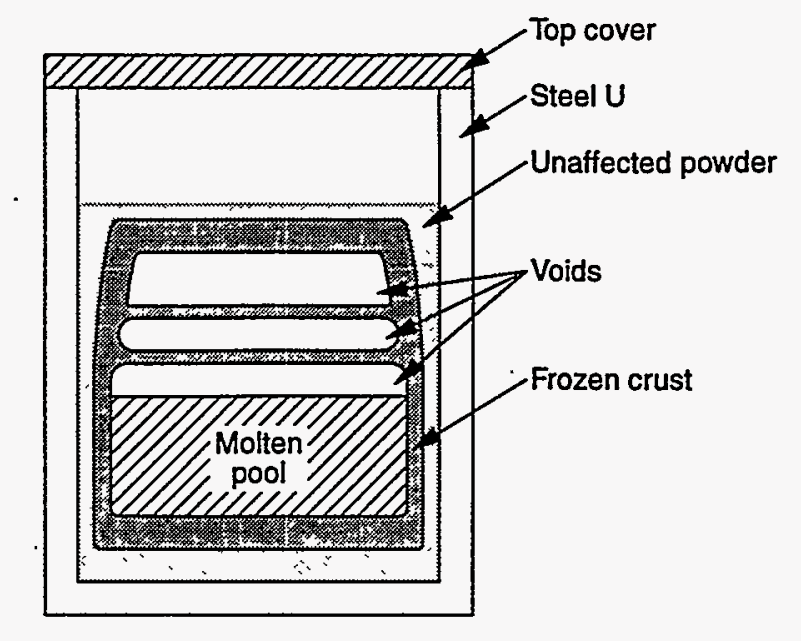

Figure A-22. Typical configuration for molten pool and surroundings based on post-test examinations.

Figure A-23 summarizes heat transfer results obtained from these tests. As illustrated in this figure, upwards and downwards heat fluxes are considerably less than sideward heat fluxes. Results in Reference A-22 indicate that upwards heat fluxes were typically a factor of 2 to 14 less and downwards heat fluxes are typically a factor of 2 to 4.5 greater than those predicted using purely convective heat transfer models. The much lower upwards heat fluxes were attributed to the solid $\mathrm{UO}_{2}$ crusts containing voids formed on top of the pool acting as insulating radiation shields and decreasing upward heat transfer. Several possible explanations for enhanced downward heat transfer have been offered. In Reference A-23, calculations were performed assuming that heat transfer within the melt is increased because of thermal radiation within the melt. Although results from these calculations yielded higher downward heat fluxes than the experimental values, the calculated values with the radiation model were closer (typically within 50\%) than the calculated values with the convection model to the experimental values. Another possible explanation for the differences between model predictions and the experimental results has been offered by Vossebrecker in Reference A-25. Vossebrecker suggests that the method of calculating the electrical heating of the $\mathrm{UO}_{2}$ led to uncertainties in the electrical conductivity of the solid debris crust and erroneous results. 


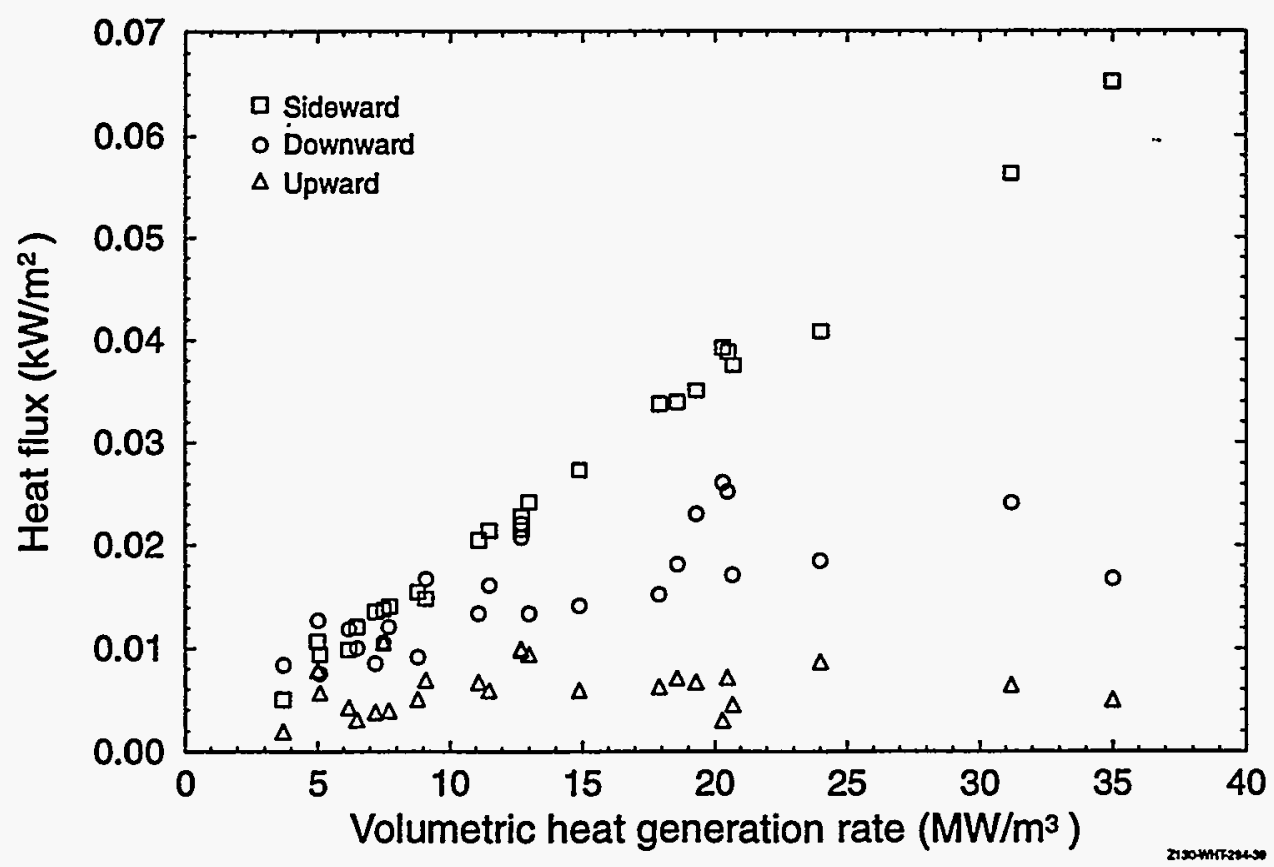

Figure A-23. Summary of heat transfer results from $\mathrm{UO}_{2}$ molten pool tests.

\section{A4.2 Recommendations}

As discussed above in Section A4.1, there are limited data for molten pools containing prototypic materials and these data were obtained by the same researchers with similar test facilities. However, significant differences occur causing reduced upward heat fluxes and enhanced downward heat fluxes. The formation of voids in the crust surrounding the pool in these tests is another important phenomena that should be addressed by SCDAP/RELAP5/MOD3.1 crust heat transfer models.

Results from experiments with prototypic results are considerably different from those using nonprototypic fluids. As illustrated in Figure A-24, experiments with prototypic materials (by Stein) ${ }^{A-23}$ exhibit much different heat flux ratios than experiments from non-prototypic materials (the Section A2.3 recommended correlations). The ratios for the recommended correlations are approximately equal to 2 with the ratio of the upward to downward heat fluxes being slightly higher than the sideward to downward heat fluxes. Conversely, the Stein data indicate that the sideward to downward heat flux ratio is higher than the upward to downward ratio. Although there is considerable scatter in the Stein data, it appears that the ratio of the upward to downward heat fluxes is generally less than unity; whereas the ratio of the sideward to downward heat fluxes is typically greater than unity. 


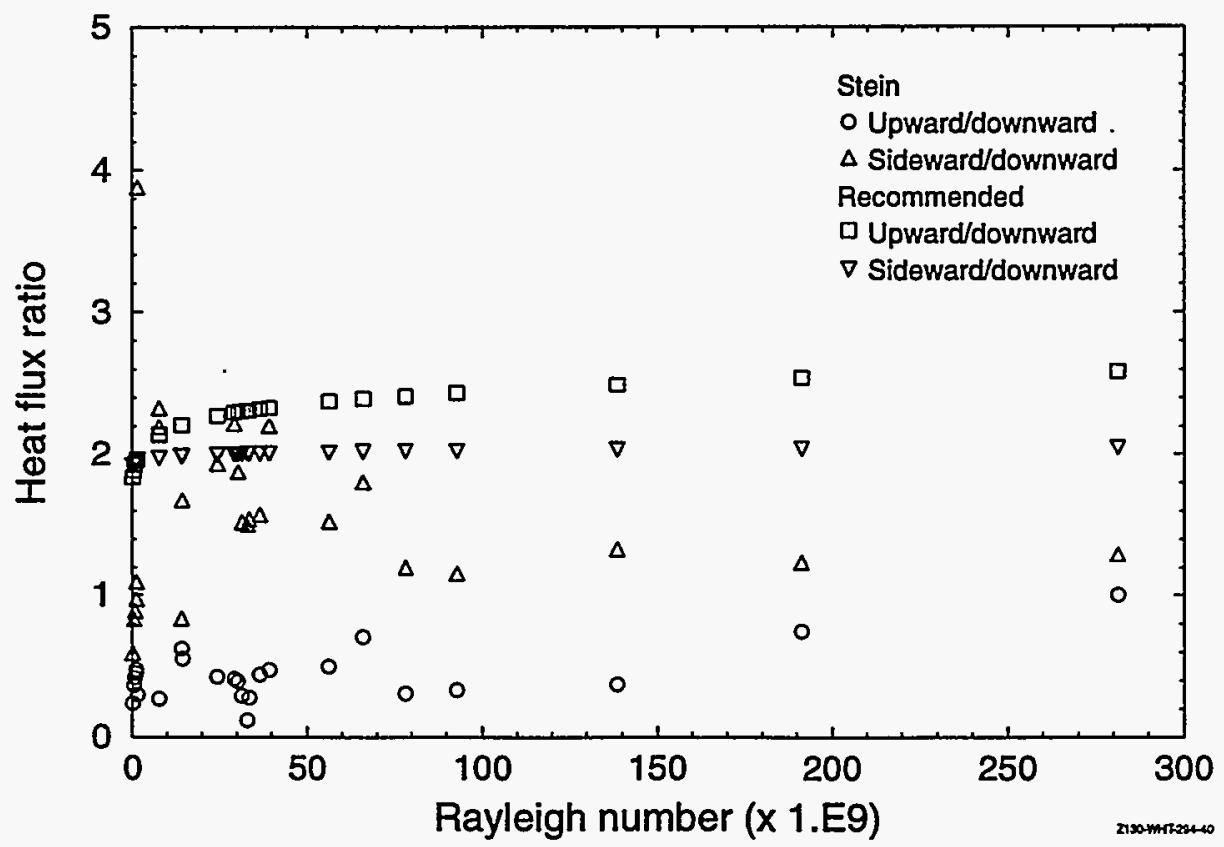

Figure A-24. Comparison of Stein data with prototypical fluids with Section A2.3 recommended correlations based on data with non-prototypic fluids.

At this time there are insufficient data to analytically explain the differences obtained from these tests and tests obtained with aqueous solutions. If these differences are due to problems associated with estimating the debris heating rate, then the correlations should not be modified based on these tests. If these differences may be explained by including internal radiation effects within the melt, then additional material property data are needed to adequately model the radiation contribution to the effective thermal conductivity of $\mathrm{UO}_{2}$. Note that some of the OECD-sponsored RASPLAV tests currently planned by the Russian Research Institute (Reference A-26) will use prototypic materials.

In summary, the limited evidence available at this time makes it difficult to recommend any modeling revisions to reflect the results observed in the limited number of tests performed with prototypic material. However, the available data indicate that additional tests with prototypic materials are needed, and when such data are obtained, molten pool models in SCDAP/RELAP5 should be reevaluated.

\section{A5. VAPOR TRANSPORT}

If the temperature in the molten pool exceeds the boiling temperatures of one or more of the metallic or oxidic components, vaporization and pool boiling occurs. In addition, the presence of fission products in molten pools formed during severe accidents may lead to gases bubbling up through the melt. There is a limited amount of experimental and theoretical work available in the literature to investigate the effect of boiling on heat transfer from a fluid with internal heating. This work has shown that these pools may be described by the three main regions shown in Figure A-25: (1) a central boiling region, (2) a lower subcooled region, and, (3) a boundary layer on the side walls. In the central region, both pool boiling and thermal expansion of the fluid generate a density difference between the bulk of the fluid and the fluid near 
the wall. This density difference provides buoyancy forces that cause an upward movement of the fluid. The vapor rising through the fluid drags fluid elements upwards. At the pool upper surface, part of the vapor escapes and the remainder condenses back into the liquid.

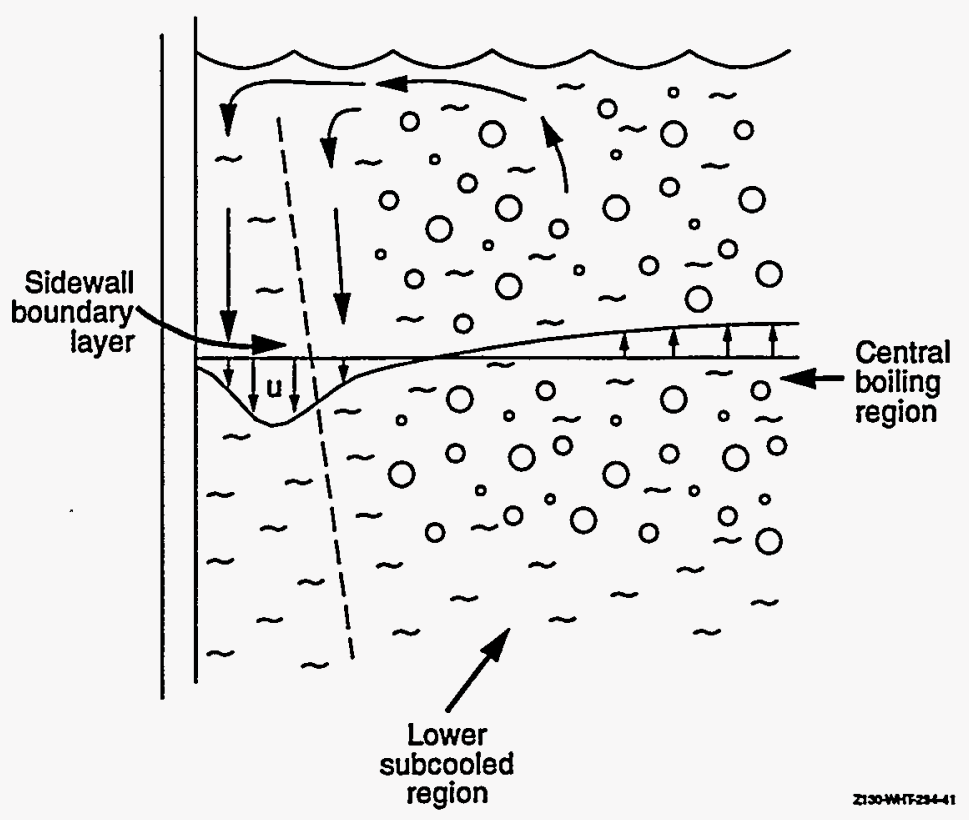

Figure A-25. Three regions or layers within a boiling pool.

Most studies performed to investigate convective heat transfer with vapor transport concentrated on the heat transfer to side boundaries of the pool. Earlier attempts to model the heat transfer associated with boiling pools attempted to describe this heat transfer as combined natural convection (due to the density differences in the fluid) and forced convection (due to the vapor lift associated with boiling). Subsequent works propose that the heat transfer could be better described as enhanced natural convection. These correlations were found to agree not only with the more recently obtained data, but also with data obtained from earlier studies. However, these studies are limited because they only concentrate on heat transfer to sidewalls. Therefore, it is recommended that a correlation based on horizontal heat transfer at liquid/liquid interfaces subjected to a gas flux be applied for predicting upward and downward heat transfer. This recommended approach is similar to the method currently used in the CORCON-MOD2 computer program $^{\mathrm{A}-27}$ to predict heat losses from a molten pool containing gases due to concrete decomposition.

\section{A5.1 Experimental and Analytical Investigations}

\section{A5.1.1 Gabor, Baker, Cassulo, and Monsieur}

Reference A-28 reports results from experiments investigating internally-heated boiling heat transfer in copper sulfate or zinc sulfate solutions contained in rectangular cavities with two vertical electrodes. The sides of the container were constructed from either double plated Pyrex glass or Lucite, and the base was constructed of copper. Copper cooling coils were attached to the electrodes and base plate to provide 
cooling. In addition, cooling was also provided on the upper surface in some experiments by using a copper tube electrically insulated with plastic shrink tubing.

Sidewards heat transfer data for a wide range of pool aspect ratios were correlated with a Reynold's number based on the superficial vapor velocity using equations with the following form:

$\mathrm{Nu}_{\mathrm{s}}=\mathrm{CPr}^{\mathrm{n}} \mathrm{Re}^{0.5}$

where

$\begin{array}{lll}\operatorname{Pr} & = & \text { Prandtl number } \\ \operatorname{Re} & = & \text { Reynold's number }=\frac{L_{s} \rho}{\mu} \\ v_{s} & = & \text { superficial vapor velocity } \\ \mathrm{L} & = & \text { pool height } \\ \mathrm{C}, \mathrm{n} & = & \text { constants. }\end{array}$

Two heat transfer correlations were obtained assuming that different mechanisms dominate heat transfer. For cases where it is assumed that forced convection due to vapor generation and a laminar boundary layer is present, the experimental data yielded values for $\mathrm{C}$ and $\mathbf{n}$ in Equation (A-70) of $2.131 \pm$ 0.121 and $1 / 3$, respectively, with $95 \%$ confidence. For higher heating rates, the central bubbly layer was thicker resulting in turbulent lateral penetrations to the pool boundaries. Using the turbulent penetration model, the experimental data yielded values for $C$ and $n$ in Equation (A-70) of $1.767 \pm 0.101$ and 1/2, respectively, with $95 \%$ confidence.

Experimental data were also used to estimate the heat transfer to the bottom surface. For boiling heat fluxes above $0.13 \mathrm{MW} / \mathrm{m}^{2}$, data indicate that the ratio of the horizontal heat flux to the downward heat flux is 3.8 .

\section{A5.1.2 Gustavson, Chen, and Kazimi}

References A-29 and A-30 report results from experimental and analytical studies to investigate horizontal heat transfer from an internally heated boiling pool. The model assumes that heat transfer can be modeled assuming that combined natural and forced convection flows are present. The model is compared with experimental results obtained by Joule heating a zinc sulfate solution in a rectangular Pyrex tank.

The model was developed by combining forced and natural convection contributions to heat transfer using the following form: 


$$
\frac{N u_{s}}{N u_{s}^{N C}}=\left[1+\left(\frac{N u_{s}^{F C}}{N u_{s}^{N C}}\right)^{m}\right.
$$

where

$$
\begin{array}{lll}
\mathrm{Nu}_{\mathrm{s}} & = & \text { total effective Nusselt number for the combined sidewards heat transfer } \\
\mathrm{Nu}_{\mathrm{s}} \mathrm{NC} & =\text { natural convection Nusselt number } \\
\mathrm{Nu}_{\mathrm{s}} \mathrm{FC} & \quad \text { forced convection Nusselt number } \\
\mathrm{n} & =\text { experimentally determined constant }
\end{array}
$$

where average turbulent forced and natural convection Nusselt numbers to a vertical wall of length, $\mathrm{L}$, are given by

$$
\begin{aligned}
& \mathrm{Nu}_{\mathrm{s}, \mathrm{L}}^{\mathrm{FC}}=0.036 \operatorname{Pr}^{1 / 3} \operatorname{Re}_{\mathrm{L}}^{4 / 5} ; \operatorname{Re}_{\mathrm{L}}>3 \cdot 10^{5} \\
& \mathrm{Nu}_{s, \mathrm{~L}}^{\mathrm{FC}}=0.0664\left(\operatorname{PrRe}_{\mathrm{L}}\right)^{1 / 2} ; \operatorname{Re}_{\mathrm{L}}<3 \cdot 10^{5} \\
& \mathrm{Nu}_{\mathrm{s}, \mathrm{L}}^{\mathrm{NC}}=0.0246\left(\mathrm{Gr}_{\mathrm{L}}\right)^{2 / 5}(\mathrm{Pr})^{7 / 15}\left(1+0.494 \mathrm{Pr}^{2 / 3}\right)^{-2 / 5} ; \mathrm{Gr}_{\mathrm{L}} \operatorname{Pr}>10^{9} \\
& \mathrm{Nu}_{\mathrm{s}, \mathrm{L}}^{\mathrm{NC}}=0.547\left(\mathrm{Gr}_{\mathrm{L}} \mathrm{Pr}\right)^{1 / 4} ; \mathrm{Gr}_{\mathrm{L}} \operatorname{Pr}<10^{9}
\end{aligned}
$$

where the Grashof, Gr, and Reynolds, Re, numbers are given by

$$
G r_{L}=\frac{g L^{3}}{v}\left[\frac{\rho_{0}-\bar{\rho}_{B}}{\rho_{0}}\right]
$$

$\operatorname{Re}_{L}=\frac{V_{c} L}{V}$

where

$$
\begin{array}{lll}
\mathrm{L} & = & \text { vertical wall length } \\
\rho_{0} & = & \text { density of the fluid near the side walls }
\end{array}
$$




\begin{tabular}{|c|c|c|}
\hline $\bar{\rho}_{\mathrm{B}}$ & $=$ & average density of the liquid and vapor in the bulk of the pool \\
\hline & $=$ & $(1-\bar{\alpha}) \rho_{1}$ \\
\hline $\bar{\alpha}$ & $=$ & average bulk void fraction $=1-1-\left(\frac{\lambda \rho_{\mathrm{v}} \mathrm{BV}}{\mathrm{LG} \mathrm{G}_{\mathrm{v}}}\right)\left(1-\exp \left(\frac{-\mathrm{G}_{\mathrm{v}} \mathrm{L}}{\lambda \rho_{\mathrm{v}} \mathrm{BV} \mathrm{V}_{\infty}}\right)\right)$ \\
\hline$\lambda$ & $=$ & latent heat of vaporization for the fluid \\
\hline$\rho_{\mathbf{v}}$ & $=$ & vapor density of the bulk fluid \\
\hline B & $=$ & experimentally determined constant \\
\hline $\mathrm{G}_{\mathrm{v}}$ & $=$ & heat generation rate in the pool per unit liquid volume $=(1-\bar{\alpha}) q^{\prime \prime \prime}$ \\
\hline $\mathrm{V}_{\infty}$ & $=$ & bubble terminal rise velocity \\
\hline$v_{c}$ & $=$ & $\begin{array}{l}\text { characteristic velocity (either the average liquid velocity or the superficial vapor } \\
\text { velocity at the upper pool surface, choice is based on success in predicting } \\
\text { experimental data). }\end{array}$ \\
\hline
\end{tabular}

Physical properties in the above equations are evaluated at the temperature $\left(T_{w}+T_{B}\right) / 2$.

The authors first compared model predictions with the data obtained from Gabor et al in Reference A-28. Reasonable agreement was obtained with the model evaluated at average void fractions reported in the experiments and $n=0.7$. Hence, the authors concluded that this good agreement confirmed the basic hypothesis of their proposed phenomenological model.

The authors then conducted their own experiments by Joule heating a zinc sulfate aqueous solution in a Pyrex glass container with dimensions of $0.17 \mathrm{~m} \mathrm{x} 0.23 \times 0.32 \mathrm{~m}$. Two copper electrodes, which were placed opposite to the wider sides of the container, were cooled by water flowing inside copper tubes that were soldered to the back of the electrodes. An aluminum test plate, which was $0.025 \mathrm{~m} \mathrm{x} 0.127 \mathrm{~m} \times 0.33$ $\mathrm{m}$, was inserted in the container. To obtain local heat transfer data, the test plate was divided along its height into thirteen equal segments that were thermally isolated by a $0.02 \mathrm{~m}$ cut into the plane facing the boiling pool. Each segment was individually cooled by water flow.

In operating the experiments, it was observed that two different flow regimes occurred - a "dense" regime and a "foaming" regime. When comparing the model predictions with "dense" regime data, the authors found good agreement with measured values for the average void fraction and $n=0.7$. However, agreement was even better if the experimental constant, $\mathrm{n}$, was assumed as unity. When comparing model predictions with "foaming" regime data, the authors found that the average Nusselt numbers for boiling pools were slightly higher than model predictions, and the authors postulated that condensations of the foam mixture at the cold wall enhanced the heat transfer process. Noting that applications for reactor safety may lead to boiling pools in the foam regime, the authors recommended that additional investigation be performed to study this regime. 


\section{A5.1.3 Kazimi}

Reference A-31 uses analytically and experimentally developed relationships for predicting heat fluxes from a pool to investigate the decay heat levels required to sustain a steady-state boiling condition and to estimate the ratios of heat losses from various surfaces of a pool. In this paper, it is assumed that molten pool containing $\mathrm{UO}_{2}$ is cylindrically-shaped (with radius, $\mathrm{R}$, and height, $\mathrm{L}$ ), thermodynamically homogeneous, with the liquid and vapor in equilibrium. Hence, uniform temperature and pressure exist in the two-phase region of the pool. The steady-state heat balance for the pool is

$$
\frac{Q}{\pi R^{2} L}=(1-\bar{\alpha}) q^{\prime \prime \prime}=\frac{2 q^{\prime \prime} s}{R}+\frac{q^{\prime \prime}}{L}+\frac{q^{\prime \prime}}{L}
$$

where

$$
\begin{array}{lll}
\mathrm{q}^{\prime \prime \prime} & = & \text { volumetric heat generation rate per unit volume of liquid } \\
\mathrm{Q} & = & \text { total heat generated within the pool } \\
\bar{\alpha} & = & \text { average void fraction } \\
\mathrm{q}^{\prime \prime} & = & \begin{array}{l}
\text { heat flux downward, sideward, or upward (designated by the subscript, d, s, or } \\
\text { u, respectively). }
\end{array}
\end{array}
$$

The sideward heat flux was estimated using the following relationship, which was based on results from the Gustavson, Kazimi, and Chen experiments discussed in Section A5.1.2 [Equation (A-71) with $\mathrm{n}=1]$.

$q^{\prime \prime}=\frac{k\left(T_{v}-T_{m}\right)}{L}\left(N u_{s}^{N C}+N u_{s}^{F C}\right)$

where

$$
\begin{array}{lll}
\mathrm{k} & = & \text { thermal conductivity } \\
\mathrm{T}_{\mathrm{v}} & = & \text { saturation temperature of the two phase region } \\
\mathrm{T}_{\mathrm{m}} & = & \mathrm{UO}_{2} \text { melting temperature }
\end{array}
$$

and the forced and natural convection Nusselt numbers were evaluated using Equations (A-72) and (A-74), respectively. For this application, the characteristic velocity in the Reynolds number was assumed as the superficial vapor velocity at the upper pool surface.

The downward heat flux was correlated based on data obtained in the Gabor experiments discussed in Section A5.1.1. It was found that the data could be correlated using the following relationship 
$q^{\prime \prime}{ }_{d}=\left[k\left(T_{v}-T_{m}\right) q^{\prime \prime \prime}\right]^{1 / 2}(1-\bar{\alpha}) f\left(V_{s}\right)$

where

$f\left(V_{s}\right)=\exp \left[0.588+0.106 V_{s}+0.0027 V_{s}^{2}\right] ; V_{s}<V_{\infty}$

$f\left(V_{s}\right)=0.6206 ; V_{s}>V_{\infty}$

where $\mathrm{V}_{\infty}$, the terminal vapor bubble velocity in a stagnant liquid, is given by

$\mathrm{V}_{\infty}=1.4\left[\frac{\sigma g\left(\rho_{1}-\rho_{\mathrm{v}}\right)}{\rho_{1}^{2}}\right]^{0.25}$

As indicated below, the upwards heat flux is assumed to be solely dependent on the vapor reaching the upper surface of the two-phase pool

$\mathrm{q}_{\mathrm{u}}=\mathrm{v}_{\mathrm{s}} \rho_{\mathrm{v}} \lambda$

where $\lambda$, represents the latent heat of vaporization of the fluid, and $\rho_{v}$ represents the vapor density.

For open-top internally boiling pools, the value of $\mathrm{V}_{\mathrm{s}}$ can be determined for a given value of $\bar{\alpha}$ and flow regime. Noting that the value of $\bar{\alpha}$ is smallest in a churn turbulent regime, Kazimi assumed churn-turbulent conditions and applied the following relations obtained from his work with Gustavson and Chen (see Section A5.1.2).

$\mathrm{V}_{\mathrm{s}, \min }=\frac{\bar{\alpha}}{1-\bar{\alpha}} \mathrm{V}_{\infty}$

For sealed pools, the void fraction is fixed because all the vapor reaching the upper surface has to be condensed there. Therefore, the maximum allowable $\mathrm{V}_{\mathrm{S}}$ at the lower surface of the top crust is that of the limiting condensation heat flux at the lower boundary of a melting solid. Based on work obtained by Gertsman and Griffith [(3-32)] and Cho and Epstein[(3-33)], Kazimi estimated the value of $V_{s}$ using the following relationships

$\mathrm{V}_{\mathrm{s}, \max }=\frac{\mathrm{q}_{\mathrm{u}, \max }}{\rho_{\mathrm{v}} \lambda}$ 
where

$$
\begin{aligned}
& \mathrm{q}^{\prime \prime}{ }_{u, \max }=\frac{\mathrm{k}\left(\mathrm{T}_{\mathrm{v}}-\mathrm{T}_{\mathrm{m}}\right)}{\left(\sigma / \rho_{1} \mathrm{~g}\right)^{1 / 2}} \overline{\mathrm{Nu}}_{\mathrm{m}} \\
& \overline{\mathrm{Nu}}_{\mathrm{m}}=0.69 \mathrm{Ra}^{0.2} ; \mathrm{Ra}<10^{8} \\
& \overline{\mathrm{Nu}}_{\mathrm{m}}=0.81 \mathrm{Ra}^{0.193} ; 10^{8}<\mathrm{Ra}<10^{10} \\
& \mathrm{Ra}=\frac{\mathrm{g}\left(\rho_{1}-\rho_{\mathrm{v}}\right) \lambda}{\mathrm{kv}\left(\mathrm{T}_{\mathrm{v}}-\mathrm{T}_{\mathrm{m}}\right)} \frac{\left(\frac{\sigma}{\rho_{1} g}\right)^{1.5}}{\left(\frac{\lambda}{\lambda_{\mathrm{f}}+\mathrm{c}_{\mathrm{p}, \mathrm{c}}\left(\mathrm{T}_{\mathrm{m}}+\mathrm{T}_{\mathrm{o}}\right)}+1\right)}
\end{aligned}
$$

where $\lambda_{f}$ is the latent heat of fusion (Note that there are some typographical errors in Reference A-31. For velocities higher than values predicted with Equation (A-86), the pool would become pressurized. For velocities lower than values predicted with Equation (A-85), the pool would undergo a change in the two-phase flow regime.

Results from applying the above correlations indicate that volumetric heat generation rates in the liquid above $12 \mathrm{MW} / \mathrm{m}^{3}$ are needed to sustain stable boiling for void fractions of 0.10 for pool radii between 0.3 and $1 \mathrm{~m}$. In general, higher heat generation rates are needed to sustain stable boiling for higher void fractions. Although the above heat generation rates are considered low for fast reactors, such power densities are high when one considers the pools that may form during a severe LWR accident. However, the presence of materials with lower vaporization temperatures and fission gases in the melt suggest that vapor enhanced heat transfer may occur at much lower power densities in molten pools that form during an LWR severe accident.

Analyses were also performed in Reference A-31 to compare the ratios of side to upward heat fluxes and downward to upward heat fluxes. Results indicate that the ratio of the sideward to upward heat fluxes is independent of pool size. For a pool void fraction of 0.1 , the upper and lower bound velocity cases yield ratios for the sideward to upward heat fluxes of 2.66 and 31.77, respectively. For a pool void fraction of 0.5 , the upper and lower bound velocity cases yield ratios for the sideward to upward heat fluxes of 3.00 and 9.048, respectively. For pool radii less than around 0.3 meters, Results indicate that the ratio of the sidewards to upwards heat fluxes were found to vary with radius. However, for pools with larger radii and a 0.1 void fraction, the upper and lower bound cases yield ratios of around 0.4 and 3.4. For pools with larger radii and a 0.5 void fraction, the upper and lower bound cases yield ratios of around 0.3 and 1.3. 


\section{A5.1.4 Greene, Jones, Schwarz, and Abuaf}

References A-34 and A-35 report results from a theoretical and experimental study of internally heated boiling pools. In their experiments, various depths of zinc sulphate solutions were Joule heated in an open tank $18 \mathrm{~cm}$ wide by $33.5 \mathrm{~cm}$ long with cooled side and bottom surfaces. Data obtained from these experiments were found to be in the two-phase bubbly flow regime. For this regime, the authors proposed that the heat transfer to the side walls be approximated by assuming an enhanced form of natural convection using the following correlations:

$\mathrm{Nu}_{s}=1.54 \mathrm{Ra}_{\mathrm{b}}^{0.25}$

for $R a_{b}<1.9 \times 10^{11}$, and

$\mathrm{Nu}_{\mathrm{s}}=0.0314 \mathrm{Ra}_{\mathrm{b}}^{0.40}$

for $R a_{b}>1.9 \times 10^{11}$, where the boiling Rayleigh number is defined by

$\mathrm{Ra}_{\mathrm{b}}=\frac{\mathrm{g} \cos \theta \alpha^{\prime} \mathrm{L}^{3}}{\alpha v}$

where $\alpha^{\prime}$ is the void fraction and $\theta$ is the angle at which the boundary is inclined from the vertical.

The authors applied the above methodology to the data obtained by Gabor, et al., (Section A5.1.1) and by Gustavson, et al. (Section A5.1.2). Correlations obtained using this method had standard deviations of \pm 0.33 or smaller, which is considerably less than deviations reported for correlations developed by Gabor, et al., and Gustavson, et al. It should be noted that the correlations developed in Reference A-34 for their new data and for the Gabor, et al. data predicted values approximately 1.5 higher than the correlation developed using the Gustavson, et al. data. It was also noted within Reference A-34 that there was considerably lower standard deviations for correlations developed using data obtained from their experiments (around 5\%) than in the data obtained by Gabor, et al., and Gustavson, et al. (21\% and 28\%, respectively).

\section{A5.1.5 Gingsberg and Greene}

References A-27 and A-36 report results from experiments performed to investigate heat transfer to a horizontal surface agitated by transverse gas flow. Experiments were performed using mercury-water fluid pairs with bubble radii ranging from 0.3 to $0.5 \mathrm{~cm}$ and superficial gas velocities ranging from 0.0 to 1.4 $\mathrm{cm} / \mathrm{s}$. Results from these tests, and some previous tests using Wood's metal and oil, were used to develop the following correlation 
$h_{u}=5.05 \frac{k}{r_{b}}\left(\frac{V_{s} r_{b}}{V_{1}}\right) \operatorname{Pr}^{0.5}$

where

$\begin{array}{ll}h_{u} & \text { heat transfer coefficient to the upper surface } \mathrm{W} / \mathrm{m}^{2} \mathrm{~K} \\ \mathrm{k} & \text { pool fluid thermal conductivity, } \mathrm{W} / \mathrm{mK} \\ \mathrm{r}_{\mathrm{b}} & \text { bubble effective radius, } \mathrm{m} \\ v_{1} & \text { pool fluid kinematic viscosity, } \mathrm{m}^{2} / \mathrm{s} \\ \operatorname{Pr} & \text { pool fluid Prandtl number } \\ V_{s} & \text { bubble superficial vapor velocity, } \mathrm{m} / \mathrm{s} .\end{array}$

Ginsberg and Greene also noted that further enhancement of heat transfer by gas entrainment effects become significant at gas superficial velocities on the order of $1 \mathrm{~cm} / \mathrm{s}$. However, analyses ${ }^{\mathrm{A}-27}$ show that this effect can be neglected because the above correlation yields sufficiently high heat transfer coefficients that the pool is almost isothermal, and heat losses from the boundary are primarily controlled by other thermal resistances, such as crust formation.

\section{A5.2 Analyses and Recommendations}

This section summarizes the recommended heat transfer correlations for modeling heat losses from a molten pool containing vapor bubbles. Calculations are performed to estimate typical heat transfer coefficients for these correlations and assess their impact on SCDAP/RELAP5 calculations.

\section{A5.2.1 Recommendations}

As discussed in Section A5.1, there has been a limited amount of research investigating the effects of vapor transport on heat losses from a volumetrically heated pool. However, analyses discussed below in Section A5.2.2 indicates that the presence of these vapors in the pool may significantly enhance heat losses from the pool. Furthermore, analyses indicate that the heat generation rates expected in pools that may form during a severe LWR accident are sufficient for stable boiling to be sustained. Therefore, it is recommended that SCDAP/RELAP5 models be modified to include the effects of boiling on heat transfer.

As discussed in Section A5.1, most experimental work has concentrated on predicting heat transfer to side walls of the pool. Of this experimental work for predicting sideward heat transfer, the methodology suggested and validated by Greene, et al. (see Section A5.1.4) appears to be the most promising. It is recommended that their correlation be applied to model horizontal heat losses because their method was found to agree with their own data as well as with the data by Gabor, et al. and because of the simplicity of their expressions for predicting heat transfer [see Equations (A-91) and (A-92)]. It is recommended that the Ginsberg and Greene correlation based on horizontal heat transfer at liquid/liquid interfaces subjected 
to a gas flux [see Equation (A-94) in Section A5.1.5] be applied for predicting upward and downward heat transfer. This recommended approach is similar to the method currently used in the CORCON-MOD2 ${ }^{A-27}$ computer program to predict heat losses from a molten pool containing gases due to concrete decomposition.

\section{A5.2.2 Impact of Including Vapor Transport}

To illustrate the possible impact that boiling may have on natural convection heat transfer, the correlations developed by Greene, et al. for predicting horizontal heat losses were applied to the $80 \% \mathrm{UO}_{2}$ I $20 \% \mathrm{ZrO}_{2}$ weight fraction pool that was predicted to form in the core region in the Reference A-3 SCDAP/ RELAP5/MOD3.1 calculations discussed in Section A1.2. The heat transfer predicted by Equations (A-91) and (A-92) are compared with values predicted by Equation (A-49), which was recommended by Kymäläinen, et al..$^{A-16}$ and by Steinberner and Reineke ${ }^{A-11}$ for heat transfer to the side wall. Results, which are shown below in Figure A-26, indicate that heat transfer may be increased by more than an order of magnitude, depending on the Rayleigh number and the void fraction within the pool. For example, if one considers the pool Rayleigh number of $10^{16}$ predicted in the Reference A-3 calculations described in Section A1.2, and assumes the void fraction for the melt corresponds to the $20 \%$ porosity estimated for samples of debris material found in the TMI-2 lower head (Reference A-43), the heat transfer to the side walls is estimated to increase by a factor of nearly 30 .

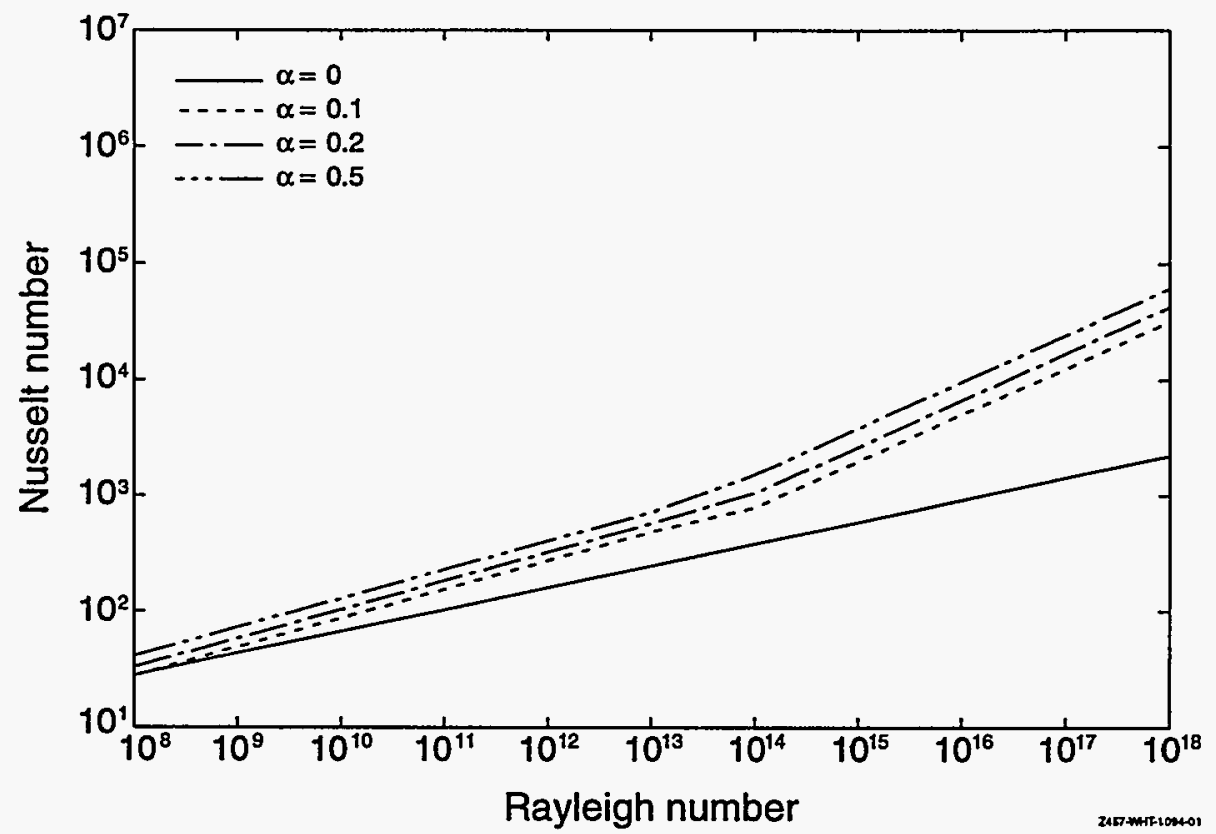

Figure A-26. Impact of boiling on heat transfer from a molten pool.

\section{A5.2.3 Directional Heat Transfer Ratios}

As discussed above, it is recommended that the Ginsberg and Green correlation be used to estimate upward and downward heat transfer from a molten pool containing vapors. Using the recommended 
correlations discussed in Section A5.2.1, estimates were obtained for the ratio of the sideward to upward heat transfer coefficient, which is equal to the ratio of the sideward to downward heat transfer coefficient. Calculations were performed for a molten pool, containing primarily $\mathrm{UO}_{2}$ for a range of pool void fractions, a range of bubble compositions, and minimum and maximum pool vapor bubble radii $(0.0012$ and $0.0431 \mathrm{~m}) .{ }^{A-36}$ In estimating the vapor velocity, $V_{s}$, relationships between the vapor velocity and the terminal vapor bubble velocity, $V_{\infty}$, proposed by Greene, et al. (see Section A5.1.4) were applied for various flow regimes. For incipient boiling $(0.00 \leq \bar{\alpha} \leq 0.03)$, bubble nucleation began at a threshold value of $V_{s} / V_{\infty}=0.2$. For bubbly flow $(0.03 \leq \bar{\alpha} \leq 0.6), 0.8 \leq V_{s} / V_{\infty} \leq 1.0$. For churn turbulent $\left(\bar{\alpha} \approx 0.4\right.$ after a peak above 0.55 ), $1.0<\mathrm{V}_{\mathrm{s}} / \mathrm{V}_{\infty}<2.0$. Results, shown in Figure A-27, indicate that the upward heat transfer coefficient will be at least a factor of two larger than the sideward heat transfer coefficient for the pool void fractions considered.

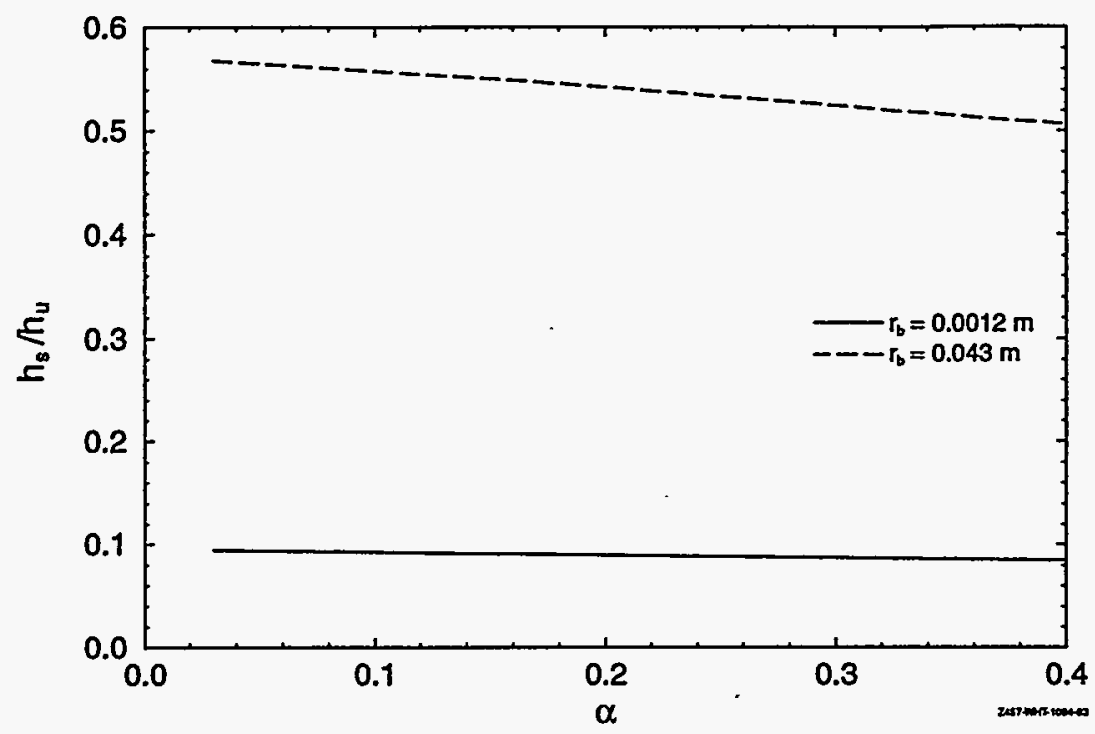

Figure A-27. Ratios of heat transfer coefficients as a function of pool void fraction.

\section{A6. MELTING AND SOLIDIFICATION}

There has been limited research on convective heat transfer from a volumetrically heated liquid undergoing a phase change. Note that convective motion within the liquid may be introduced via several mechanisms: (1) temperature and/or concentration gradients in the presence of a gravitational field (natural convection), (2) the intrinsic density differences associated with the transition between the phases and the movement of the solid-liquid front (bulk convection), and, (3) the presence of surface tension gradients at the "free" surface of the liquid (surface tension driven convection). Although the effects of natural convection on solidification and melting of metals has been recognized as significant for some time, most of the studies in this area are qualitative rather than quantitative. For example, it has been observed in the case of melting, natural convection accelerates the phase change process; whereas for freezing, natural convection decreases the rate of solidification. In addition, it has been observed that in melting, natural convection occurs regardless of whether the initial temperature of the medium is equal to or different from the fusion value. On the other hand, in the case of freezing, the initial temperature must be different from the fusion value in order for natural convection to occur. Finally, it should be noted that these studies were 
primarily directed toward establishing that convection is needed to accurately predict melting/ solidification heat transfer phenomena, rather than the previously assumed conduction heat transfer.

In this section, some representative studies in this area are reviewed. Results from these studies are sufficient to identify several important effects of melting and solidification on heat transfer from a molten pool. However, it is not felt that there are sufficient data and studies in this area to warrant that any additional SCDAP/RELAP5 model modifications be recommended.

\section{A6.1 Experimental and Analytical Investigations}

In this section, several representative studies applicable to melting and solidification in a molten pool are discussed below. As indicated by the studies discussed below, most work in this area considered melting rather than solidification and no work was found using fluids with volumetric heating. The studies discussed in this section were selected because their results illustrate important points to consider in modeling heat transfer from a molten pool with solidification and melting or because they presented quantitative results from which correlations for predicting heat transfer were obtained. Additional studies in this area are summarized in Reference A-44.

\section{A6.1.1 White, Bathelt, Leidenfrost, and Viskanta}

Several experimenters (References A-45, A-46, and A-47) have studied the variation with time of the size and shape of the melt region around one or more electrically-heated horizontal cylinders embedded in solid paraffin. Results indicate that for a short period after the onset of melting, that heat transfer is primarily by conduction, and the moving interface is represented by a succession of concentric circles. As natural convection develops in the melt, the solid-liquid interface becomes asymmetrical about the axis of the cylinders. Thermal plumes form above the heaters and convey hot liquid to the upper portion of the melt regions and cause additional melting. The rapid cooling of the plumes as they impinge at the top of the melt cavities reduces natural circulation around the sides and bottom of the cavity. As a result, melting occurs mainly in a region above the cylinders, with relatively little melting below, and the solid liquid interface becomes somewhat pear-shaped (see Figure A-28). In multiple cylinder tests, horizontal spreading was found to dominate in tests with tighter spacing between cylinders, whereas vertical spreading was found to dominate in tests with wider spacing between the cylinders. Local heat transfer coefficients around the cylinder were obtained for single and multiple cylinder tests. 


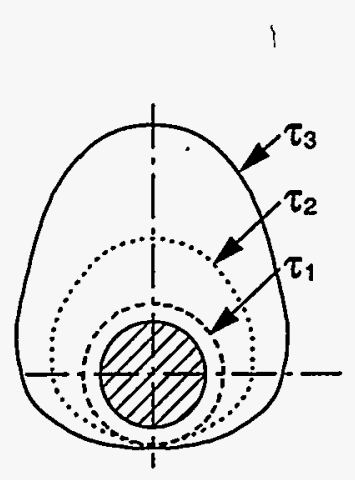

(a)

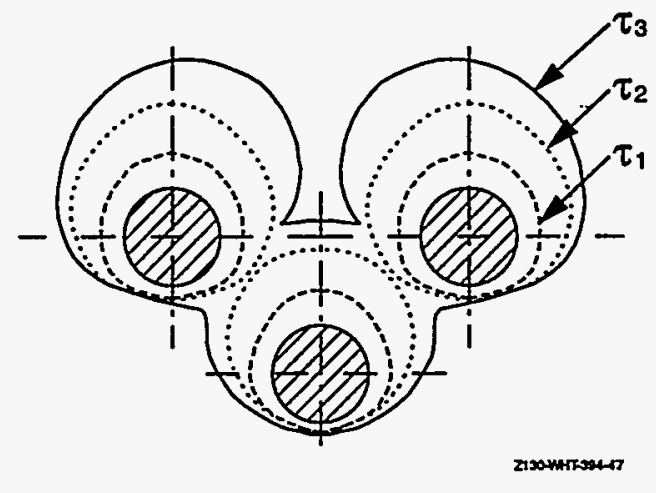

(b)

Figure A-28. Experimentally determined melt contours during melting from (a) one and (b) three uniformly heated cylinders.

\section{A6.1.2 Ho and Viskanta}

Reference A-48 reports results from experiments investigating melting of paraffin (n-octadecane) from an isothermal vertical wall of a rectangular cavity. Local heat transfer coefficients were measured and the solid-liquid interface motion during phase change was recorded photographically. Representative results for the solid-liquid interface motion are shown below in Figure A-29. Results showed that initial melting is uniform except at the upper free surface where more melting occurs because of density-induced melt motion, resulting from the volume increase which accompanies the phase change. This phenomena forced the liquid to seek extra space to expand and thus overflow the top of the solid core. As the heating continued, the buoyancy-driven convection in the melt started to develop and maximum melting was observed along the upper surface of the heater. As the buoyancy-driven flow moved upward along the heated surface, the fluid temperature increased. The flow direction was changed by the upper free surface and, as the melt passed downward along the solid-liquid interface, it progressively cooled, resulting in much smaller melting rate near the bottom. 


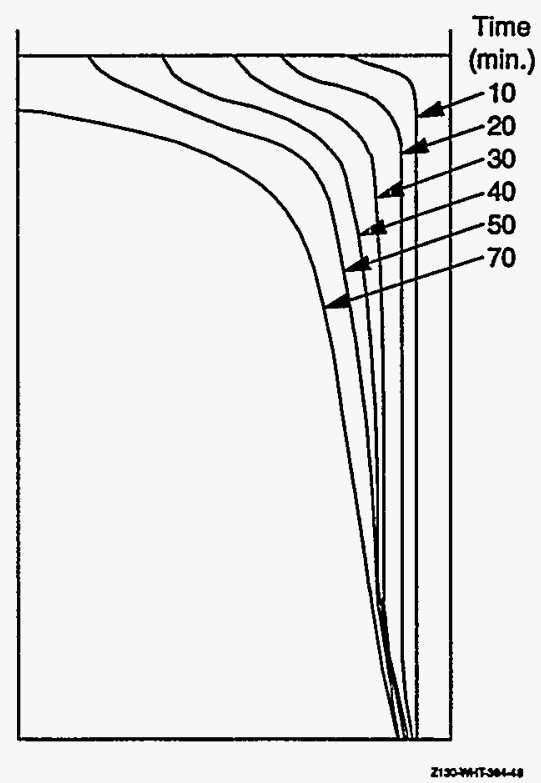

Figure A-29. Representative results indicating the solid-liquid interface position and shape.

\section{A6.1.3 Sparrow, Broadbent, and Mendes}

Studies have also been performed investigating solidification in cooled vertical copper tubes (References A-49 and A-50). It should be noted that these experiments were performed using paraffin without internal heating. Hence, natural convection within the liquid was found to play only a modest role at small times and disappear when the superheat in the liquid was dissipated. However, the manner in which the liquid was observed to solidify may significantly impact heat transfer from a molten pool with internal heat generation. During solidification, the difference in densities between the solid and liquid phases produce contractions of the solid (see Figure A-30). The increase in density that occurs as liquid solidifies causes the liquid level to drop as the freezing progresses, resulting in the sloped regions at the top of the cavity shown in Figure A-30. In the vertical tube that was open at the top of these experiments, heat losses cause a skin of solid to also form on top of the liquid (similar to the crust expected on a molten pool). Meanwhile, the layer of liquid below the skin has decreased causing a void beneath the skin. It should be noted that this behavior was also observed in the molten $\mathrm{UO}_{2}$ experiments discussed in Section A4.1, and results from these experiments indicated that the void that formed between the upper crust and the pool caused upward heat losses to be reduced. 


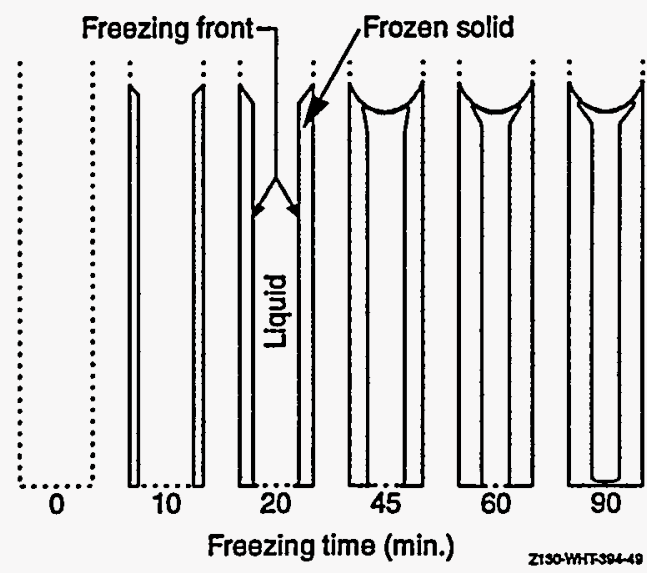

Figure A-30. Representative patterns of paraffin freezing in an open-ended vertical tube.

\section{A6.1.4 Kemink and Sparrow}

Transient and steady-state heat transfer coefficients measured for melting about a heated vertical cylinder embedded in n-eicosane paraffin are reported in Reference A-51. The experiments were conducted by placing an electrically-heated vertical cylinder at the axis of a cylindrical containment vessel housing paraffin. The containment vessel was maintained at a nearly constant temperature by immersing it in a constant temperature water bath. The investigation considered cases with the paraffin at its melting temperature and at a temperature below melting. Open-topped and closed-topped containments were used in each case (the closed-topped containment was capable of accommodating the expansion that occurred when the paraffin melted). Results indicated that there was no difference between tests with a cover imposing a no-slip boundary condition on the melt and tests bounded above by an insulating space that permitted motion of the melting fluid. Natural convection heat transfer coefficients in the presence of subcooling were found to be between 10 and $15 \%$ lower than those for the cases without subcooling.

The steady-state surface averaged coefficients were correlated using a transient Rayleigh number, $\mathrm{Ra}^{\prime}$, with the cylinder length as the characteristic dimension. The results for no subcooling and subcooling cases were correlated, respectively, by the following empirical equations

$\mathrm{Nu}=0.393 \mathrm{Ra}{ }^{0.267}$ without subcooling

$\mathrm{Nu}=0.096 \mathrm{Ra}^{10.323}$ with subcooling

for transient Rayleigh numbers between $10^{9}$ and $2 \times 10^{10}$. These relationships are compared below in Figure A-31 to a transient version of (A-48) from Section A2.2.3, which is the empirically-obtained equation recommended in this study or predicting sideward heat losses (see Section A2.3 and Section A3.2). As illustrated below, heat transfer for cases without subcooling in the melt is similar to values predicted with the recommended correlation. However, results also indicate that heat transfer is 
considerably reduced for cases with subcooling in the melt. Finally, it should be noted that the Kamink and Sparrow correlations were obtained for fluids with Rayleigh numbers considerably lower than values expected for severe accident conditions.

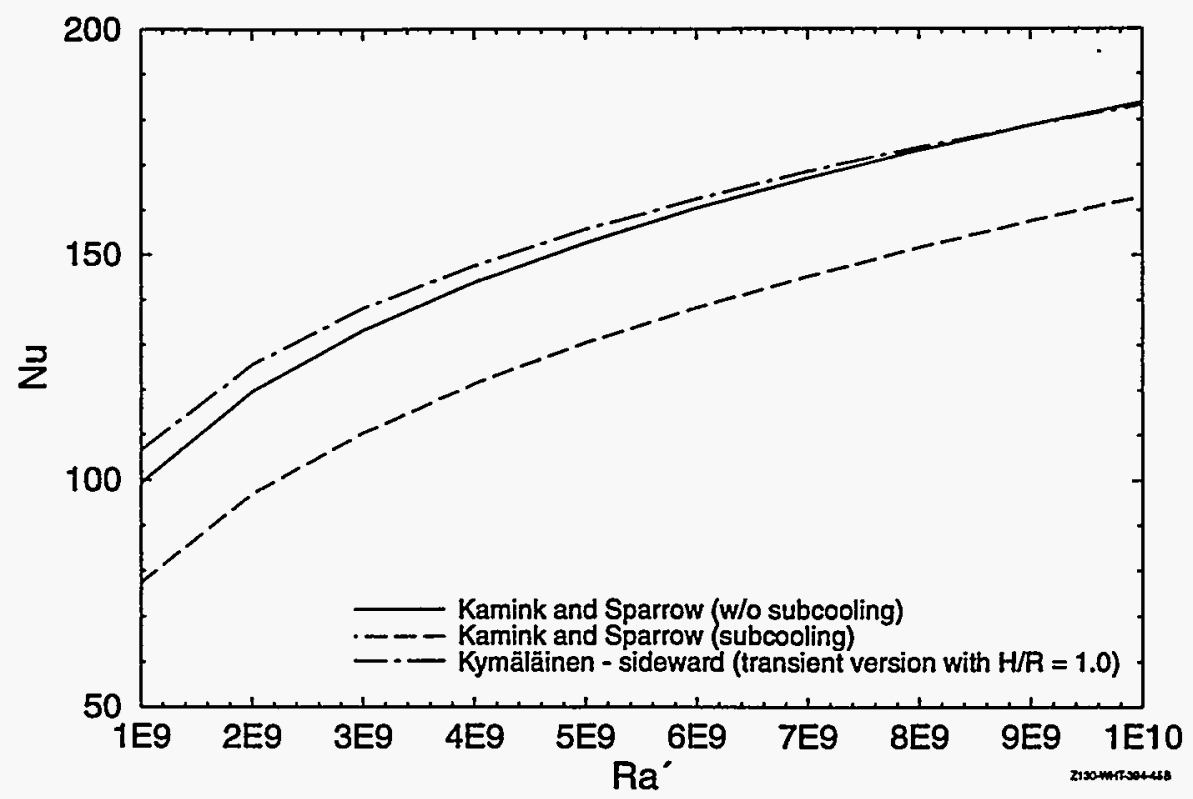

Figure A-31. Comparison of Kemink and Sparrow correlations with Section A2.3 recommended correlation for sideward heat transfer.

\section{A6.2 Summary and Recommendations}

As illustrated by the studies reviewed in this section, natural convection will significantly impact the manner in which melting and solidification occurs in a molten pool during a severe accident. The motion in the fluid will enhance heat fluxes (and accelerate melting or decrease solidification) along upper portions of the side walls and the upper walls. Furthermore, data indicate that several phenomena associated with solidification and melting will increase fluid motion within the liquid, such as the increase in fluid volume associated with melting. In this review, limited amounts of quantitative results were found for predicting heat transfer along the melting or solidifying surfaces. As indicated within this section, the data were obtained for a limited range of conditions that were significantly different than those expected for a molten pool during a severe accident. Therefore, it is not recommended that these correlations be incorporated into SCDAP/RELAP5 molten pool models.

An important finding from solidification experiments discussed in this area was the observation that voids between the upper surface of the melt and the upper crust may form due to the decrease in volume associated with melt solidification. This finding was consistent with observations from molten $\mathrm{UO}_{2}$ experiments discussed in Section A4.1. It may be important in predicting heat transfer from a molten pool because the presence of these voids will suppress upward heat losses from the pool. However, it is felt that additional experiments are needed to investigate crust stability and scale effects before this phenomenon is included in SCDAP/RELAP5 molten pool models. 
In summary, there are several phenomena associated with melting and solidification that are currently included in SCDAP/RELAP5 models. For example, the ability of crusts to form and melt due to heat transfer from the pool is included in SCDAP/RELAP5 models. Furthermore, the change in volume associated with melting and solidification is also considered, although the model assumes that the pool collapses rather than solidifies with voids in it. SCDAP/RELAP5 models do not include any enhanced fluid motion due to melting and solidification phenomena. The models also do not consider the potential for solids to become entrained in the melt and the effects of these entrained solids on heat transfer from the pool. At this time, it is recommended that additional numerical and experimental studies be performed to investigate the above phenomena before modifications are proposed for SCDAP/RELAP5 molten pool models.

\section{A7. MULTIPLE COMPONENT/LAYER POOLS}

It has been postulated that multiple component pools may form during a severe accident. For example, there is the potential that control and structural material may relocate earlier than ceramic material or that radiation heat transfer from a large, ceramic pool may cause lower plenum structural material to relocate on top of the ceramic material. When modeling these multiple component pools, it is necessary to determine if there will be natural convection in one or both of the layers. Furthermore, it is necessary to understand what, if any, material interactions are possible that could result in a single homogeneous layer. If it is determined that the layers are immiscible, it is necessary to determine if a crust will form between the layers and estimate the effect of this crust on heat transfer between the layers.

A limited amount of experimental and analytical work has been performed to investigate multiple convecting layers with internal heat sources. As discussed within this section, experimental studies only considered internal heating in the lower layer. Previous experimental and analytical studies investigating convection in multiple layer pools are summarized in Section A7.1. As discussed within Section A7.2, it is felt that additional numerical and experimental studies are needed before any model modifications to SCDAP/RELAP5 can be recommended. However, it is important that the user understand uncertainties associated with the manner in which the current model treats multilayer pools.

\section{A7.1 Experimental and Analytical Investigations}

In this section, previous work investigating natural convection in multiple layer pools is reviewed. As discussed below, experimental investigations have considered two immiscible fluids of different density with internal heat generation in only the lower layer. Some analytical studies were performed for internal heating in both layers. Figure A-32 illustrates a typical configuration for a two-layer molten pool. Variables illustrated in Figure A-32 will be used to discuss results from multiple layer investigations. As noted in the figure, variables are consistent with variables used throughout this report except for the additional subscript, $\mathrm{T}$ or $\mathrm{B}$, to denote, top or lower layer, respectively. 


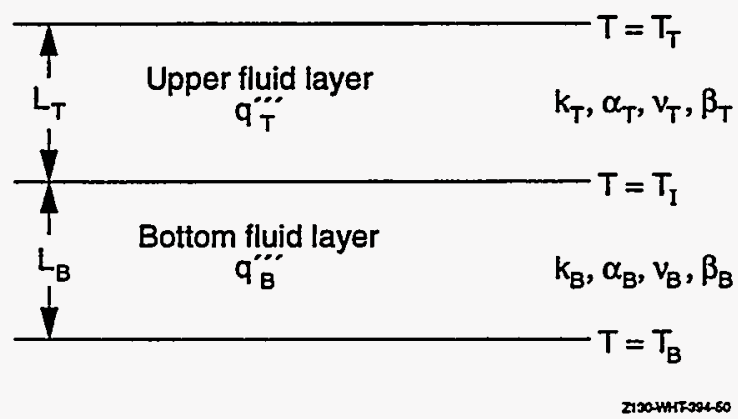

Figure A-32. Typical configuration for a two-layer molten pool.

\section{A7.1.1 Fieg}

Results from experiments investigating convection in an experiment with an internally heated fluid layer below a second unheated, immiscible fluid layer are reported in Reference A-52. In these experiments, water was used for the lower layer and heptane, which has a lower density than water, was used for the upper layer. A typical temperature profile from these experiments is shown in Figure A-33. As indicated in this figure, the data indicates that a hydrodynamically rigid interface exists between the liquids with high heat conduction. The temperature profile in the lower layer was similar to that observed in a fluid without the upper, unheated layer. The temperature profile in the upper layer was found to correspond to that of the following Benard convection correlation ${ }^{\mathrm{A}-53}$

$N u_{u}=A\left(R a^{\prime}\right)^{P}$

where the transient Rayleigh number is defined in terms of the nonheated fluid's height and thermal properties, the temperature difference between the top and bottom of the nonheated fluid, and the constants, $\mathrm{A}$ and $\mathrm{P}$, are given by

$$
\begin{aligned}
& \mathrm{A}=0.104 \mathrm{Pr}^{0.84} \\
& \mathrm{P}=0.305
\end{aligned}
$$

for $10^{5} \leq \mathrm{Ra}^{\prime} \leq 10^{9}$. 


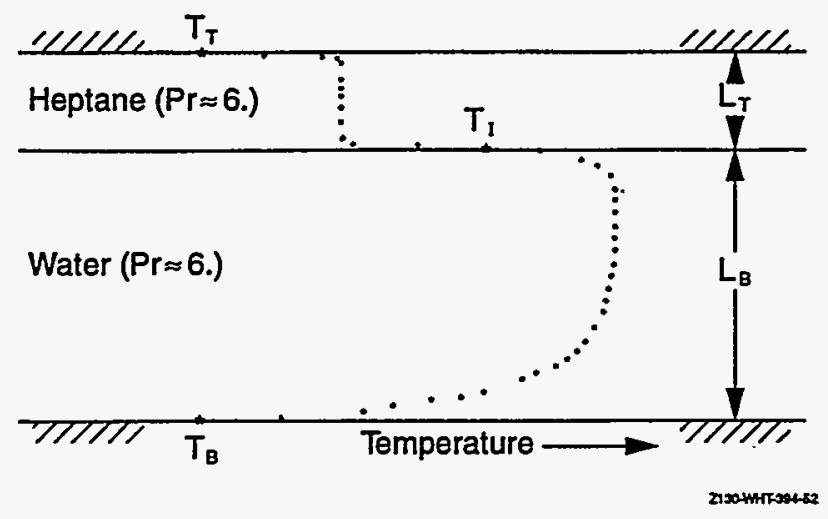

Figure A-33. Typical temperature profile observed in two stratified liquid layers (only the lower one heated).

\section{A7.1.2 Schramm and Reineke}

Results from a numerical and experimental investigation of natural convection in a rectangular channel filled with two immiscible fluids of different densities are reported in Reference A-54. The experimental investigation, which was performed to validate the computer code used in the numerical investigations, consisted of small scale tests to study cases with a higher density fluid (molten paraffin) beneath a lighter density fluid (water) and cases with a lighter density fluid (silicon oil) on top of a higher density fluid (water). In both types of cases studied, the bottom fluid was internally heated by passing electrical current through the fluid. For cases with the lighter fluid initially at the bottom, results indicated that the process was unstable. However, results for cases with the lighter fluid initially on top indicate that the two fluids remain in stratified layers. In these latter cases, conditions were varied so that the ratio of the height of the lighter (top) fluid to the total fluid height was varied between 0.11 to 0.75 . Results were similar to results obtained by Fieg (see Section A7.1.1) in that the temperatures observed in the lighter layer were similar to those expected from Bernard convection and that the temperatures observed in the heavier layer were similar to those obtained in an internally heated fluid bounded by rigid walls at the top and the bottom.

Calculations were performed to study the effect of the upper fluid's height assuming a constant height and heat generation rate in the lower fluid. Results indicate that for very small upper layers, heat will be transported through the fluid via conduction. As the upper fluid's height increases $\left(0.1<L_{T} \prime\right.$ $\left.\left(\mathrm{L}_{\mathrm{T}}+\mathrm{L}_{\mathrm{B}}\right)<0.25\right)$, convective heat transfer within the upper fluid becomes important and increases the upward heat flux from the fluid. Then, as the upper fluid's height increases further $\left(\mathrm{L}_{\mathrm{T}} /\left(\mathrm{L}_{\mathrm{T}}+\mathrm{L}_{\mathrm{B}}\right)>0.25\right)$, the upward heat transfer decreases.

\section{A7.1.3 Kulacki, Min, Nguyen, and Keyhani}

Reference A-55 and Reference A-56 report results from experiments investigating convection in two layer experiments with internal heating only present in the lower layer. The layers were bounded from 
below by an insulated surface and from above by an isothermal surface. Upper layers in these experiments were composed of silicon oil or heptane, and the lower layer was a copper sulphate solution. Various ranges of sublayer thickness were considered.

Upward heat transfer data from these experiments were correlated using an effective Nusselt number, $\mathrm{Nu}_{\mathrm{u}}^{\text {eff }}$, defined by

$\mathrm{Nu}_{u}^{\text {eff }}=\frac{h\left(\mathrm{~L}_{\mathrm{T}}+\mathrm{L}_{\mathrm{B}}\right)}{\mathrm{k}_{\mathrm{e}}}$

with

$\mathrm{k}_{\mathrm{e}}=\frac{\mathrm{L}_{\mathrm{T}}+\mathrm{L}_{\mathrm{B}}}{\mathrm{L}_{\mathrm{T}} / \mathrm{k}_{\mathrm{T}}+\mathrm{L}_{\mathrm{B}} / \mathrm{k}_{\mathrm{B}}}$

and

$h=\frac{q^{\prime \prime \prime}}{T_{T}-T_{B}}$

The above equations were used to effectively combine the two sublayers into a single layer with an averaged thermal conductivity and uniform internal heating rate. The experimentally obtained heat transfer data were correlated using a relationship of the following form

$\mathrm{Nu}_{\mathrm{u}}^{\text {eff }}=\mathrm{CRa}_{\mathrm{B}}^{\mathrm{m}}$

where values for the constants, $\mathrm{C}$ and $\mathrm{m}$, along with the range of conditions examined are summarized in Table A-5. These correlations are compared in Figure A-34 to a correlation obtained by Emara and Kulacki for predicting upward heat transfer from a single layer [Equation (A-22) of Section A2.1.4]. As illustrated below, the top layer reduces upward heat losses. Results in Figure A-34 also indicate that heat transfer upward approaches values for a single layer as the layer length ratio $\left(\mathrm{L}_{\mathrm{T}} / \mathrm{L}_{\mathrm{B}}\right)$ increases. Analyses in Reference A-56 indicate that for small layer ratios, the upper layer acts as a large thermal resistance to upwards heat transfer, which becomes conduction dominated. In fact, it is predicted that the minimum upward heat flux occurs for cases with $\mathrm{L}_{\mathrm{T}} \mathrm{L}_{\mathrm{B}}<0.03$. For higher layer ratios, convection occurs in the upper layer. Hence, upward heat transfer approaches that of a single layer.

Experimental uncertainties in the Nusselt and Rayleigh numbers were estimated to be $5.3 \%$ for both the silicone oil-water and heptane-water systems. This compound uncertainty considers uncertainties associated with quantities used to calculate physical data and dimensionless groups. 


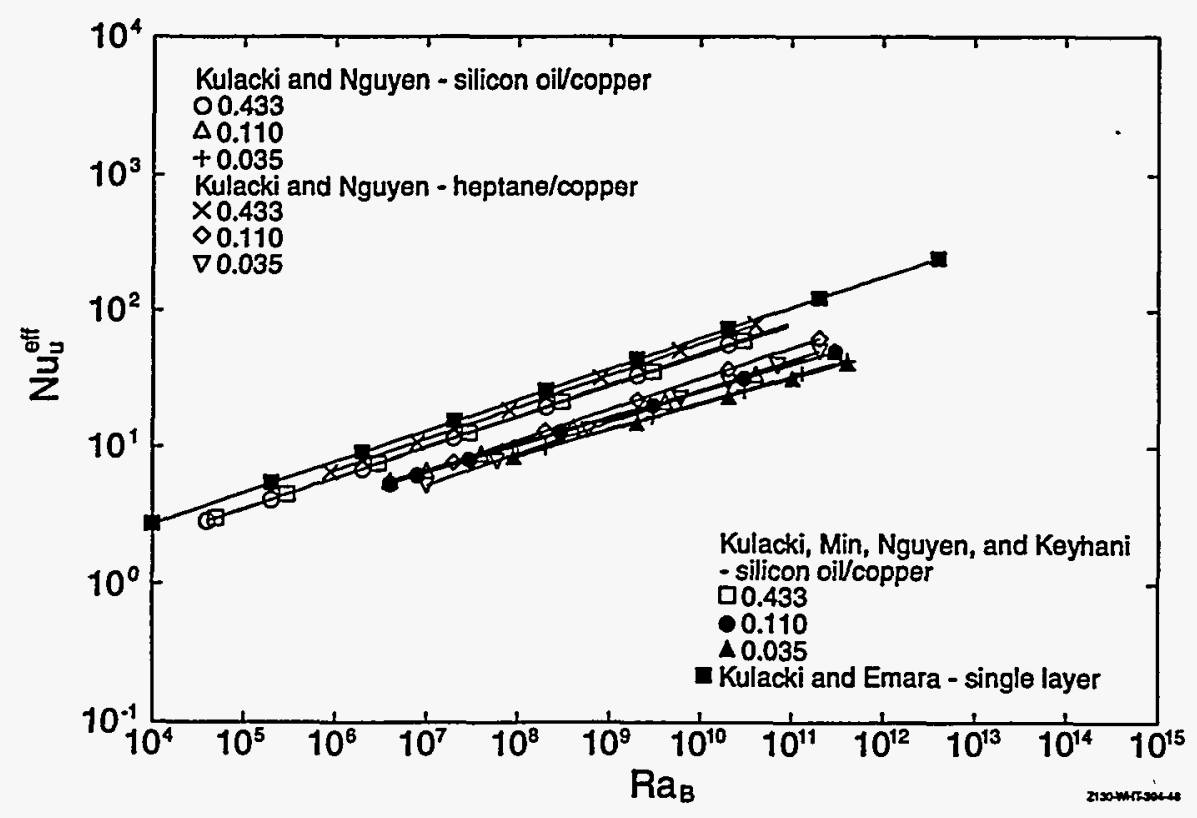

Figure A-34. Comparison of multiple-layer and single-layer correlations

\section{A7.2 Summary and Recommendations}

As discussed within Section A7.1, there has been limited research investigating convection within multiple component or multiple layer fluids. However, several investigations indicate that the presence of a nonheated layer above a heated layer reduces heat losses from the convecting layer. No experimental data were found investigating convective heat transfer in multiple layer fluids with only the top layer heated or with both layers heated. Furthermore, no data were found for modeling material interactions or the impact of crust formation. Therefore, it is recommended that additional numerical and experimental work be performed before any modifications to SCDAP/RELAP5 models are implemented. ${ }^{a}$

Nevertheless, it is important to understand how heat transfer will be predicted by SCDAP/RELAP5 when a molten pool forms containing two density-stratified layers. Currently, SCDAP/RELAP5 assumes that the volume of the molten pool corresponds to the entire volume of molten material. Using this volume, an effective pool radius is estimated that is then used to calculate the pool Rayleigh number used in steady-state heat transfer correlations. However, the heat transfer correlations are evaluated using the material properties corresponding to the material within each layer. For example, consider a molten pool with an internal heat generation rate of $1.98 \mathrm{MW} / \mathrm{m}^{3}$ consisting of a $2 \mathrm{~m}^{3}$ volume of metallic melt beneath a $4 \mathrm{~m}^{3}$ volume of ceramic melt. The effective radius of the pool calculated by SCDAP/RELAP5 would be approximately $0.95 \mathrm{~m}$. Rayleigh numbers estimated for material in the metallic and ceramic region would be $1.3 \times 10^{15}$ and $4.7 \times 10^{13}$, respectively. Heat transfer coefficients along the upper side of the pool would

a. Discussions indicate that two component, two fluid layer tests will be included in the natural convection heat transfer test program being performed at the University of California - Santa Barbara. ${ }^{\mathrm{A}-20}$ These tests will use uniform cooling to simulate internal heat generation in each layer. 
be estimated by SCDAP/RELAP5 using the correlations recommended by Mayinger, Jahn, Reineke, and Steinbrenner (see Equation (A-30) and Figure A-10 in Section A2.2.1). For the upper side of the pool, current SCDAP/RELAP5 models would estimate that the heat transfer coefficient from the metal and ceramic materials, would be 12,400 and $2,300 \mathrm{~W} / \mathrm{m}^{2} \mathrm{~K}$, respectively. There are no data with which to refute or substantiate these predictions. Hence, it is strongly recommended that additional studies be performed to investigate how this phenomena is predicted with more detailed numerical models.

Table A-5. Correlation constants and test conditions for multilayer experiments.

\begin{tabular}{|c|c|c|c|c|c|c|}
\hline \multicolumn{2}{|c|}{ Correlation Constants } & \multirow[t]{2}{*}{$\mathbf{R a}_{B}$ range } & \multirow[t]{2}{*}{$\mathbf{P r}_{\mathbf{B}} / \mathbf{P r}_{\mathbf{T}}$} & \multirow[t]{2}{*}{$\mathbf{L}_{\mathbf{T}} / \mathbf{L}_{\mathbf{B}}$} & \multirow{2}{*}{$\begin{array}{c}\text { Fluid } \\
\text { Top/ } \\
\text { bottom }\end{array}$} & \multirow[t]{2}{*}{ Reference } \\
\hline $\mathbf{C}$ & $\mathbf{m}$ & & & & & \\
\hline 0.254 & 0.228 & $\begin{array}{c}3.4 \times 10^{4} \text { to } \\
9.5 \times 10^{10}\end{array}$ & \multirow[t]{3}{*}{$7 / 84$} & 0.433 & \multirow{3}{*}{$\begin{array}{l}\text { Silicon oil/ } \\
\text { copper } \\
\text { Sulphate } \\
\text { solution }\end{array}$} & \multirow[t]{6}{*}{$\begin{array}{l}\text { Kulacki and } \\
\text { Nguyen }^{\mathrm{A}-56}\end{array}$} \\
\hline 0.286 & 0.196 & $\begin{array}{c}3.8 \times 10^{6} \text { to } \\
3.0 \times 10^{11}\end{array}$ & & 0.111 & & \\
\hline 0.244 & 0.194 & $\begin{array}{c}9.3 \times 10^{7} \text { to } \\
4.2 \times 10^{11}\end{array}$ & & 0.035 & & \\
\hline 0.269 & 0.233 & $\begin{array}{c}8.7 \times 10^{5} \text { to } \\
4.1 \times 10^{10}\end{array}$ & \multirow[t]{3}{*}{$6.5 / 6.5$} & 0.433 & \multirow{3}{*}{$\begin{array}{l}\text { Heptane/ } \\
\text { copper } \\
\text { Sulphate } \\
\text { solution }\end{array}$} & \\
\hline 0.171 & 0.227 & $\begin{array}{c}1.6 \times 10^{7} \text { to } \\
1.6 \times 10^{11}\end{array}$ & & 0.111 & & \\
\hline 0.129 & 0.230 & $\begin{array}{l}1.1 \times 10^{7} \text { to } \\
2.1 \times 10^{11}\end{array}$ & & 0.035 & & \\
\hline 0.264 & 0.225 & $\begin{array}{c}3.4 \times 10^{4} \text { to } \\
9.5 \times 10^{10}\end{array}$ & \multirow[t]{3}{*}{$6.3 / 74$} & 0.433 & \multirow{3}{*}{$\begin{array}{l}\text { Silicon oil/ } \\
\text { copper } \\
\text { Sulphate } \\
\text { solution }\end{array}$} & \multirow{3}{*}{$\begin{array}{c}\text { Kulacki, } \\
\text { Min, } \\
\text { Nguyen, and } \\
\text { Keyhani }^{\mathrm{A}-55}\end{array}$} \\
\hline 0.262 & 0.199 & $\begin{array}{c}3.8 \times 10^{6} \text { to } \\
3.1 \times 10^{11}\end{array}$ & & 0.110 & & \\
\hline 0.250 & 0.192 & $\begin{array}{c}9.3 \times 10^{7} \text { to } \\
4.3 \times 10^{11}\end{array}$ & & 0.035 & & \\
\hline
\end{tabular}

\section{A8. SCDAP/RELAP5 MODELING RECOMMENDATIONS}

Within this report several phenomena impacting convective heat transfer from a molten pool have been investigated with the objective of determining what, if any, modifications are needed to improve the SCDAP/RELAP5/MOD3.1 molten pool heat transfer models. In this section, recommendations for SCDAP/RELAP5 molten pool heat transfer models are summarized and areas where additional data are needed before revisions to SCDAP/RELAP5 heat transfer model can be recommended are identified. 
Based on this review, it is felt that there are sufficient data to warrant recommending the following modifications to the SCDAP/RELAP5 molten pool heat transfer models.

- Use steady-state heat transfer correlations obtained from the Kymäläinen, et al. COPO tests. The correlations recommended by Kymäläinen, et al. for predicting upward and downward heat transfer are similar to those used in the current SCDAP/RELAP5 model. However, it is recommended that these correlations be used because they were obtained from higher Rayleigh number tests and they are consistent with data obtained from several other two- and three-dimensional investigations. Switching to these correlations would include the use of a separate, horizontal, heat transfer coefficient and allow non-hemispherical geometries to be modeled. It is recommended that the Mayinger data for predicting local heat transfer coefficients along curved lower surface of a pool continue to be used in the SCDAP/RELAP5 model.

- Consider cylindrical and hemispherical pool shapes and aspect ratios. By mapping the volume of a molten pool onto a hemispherical shape, SCDAP/RELAP5 models may be erroneously predicting sideward heat losses. For example, scoping calculations in Section A2.3 indicate that heat transfer coefficients may vary by as much as $20 \%$ when the proper geometry of the pool is considered. Therefore, it is suggested that shape-factor selection criteria be included in SCDAP/RELAP5 to determine if the melt region is torispherical or hemispheric and the aspect ratio associated with the selected geometry. These geometrical parameters could then be used to appropriately apply the Kymäläinen, et al. COPO correlations.

- Apply steady-state correlations in their transient form. As demonstrated within Section A3.2.2, it is incorrect to apply steady-state correlations before the pool has reached a steady-state because heat transfer coefficients will be overpredicted by over a factor of 2 (and possibly orders of magnitude at early stages of the transient). Furthermore, representative results from a SCDAP/RELAP5/MOD3.1 run indicates that molten pools will be in a transient stage for most time periods of interest in a severe accident analysis. Hence, it is recommended that the steady-state Kymäläinen, et al. COPO correlations be applied using their transient forms in SCDAP/RELAP5 calculations.

- Consider the effects of vapor transport on convection heat transfer. Although additional data are needed to validate the proposed modifications, it is suggested that horizontal heat transfer be modeled using correlations proposed by Greene, et al. and that upward and downward heat transfer be modeled using the Ginsberg and Greene correlation. Note that use of this method requires selecting a boiling regime based on the average void fraction of the melt and calculating a bubble terminal vapor velocity. Furthermore, it should be noted that the volume of voids due to boiling and fission gas production must be included in estimating the melt void fraction. Scoping calculations in Section A5.2.2 indicate that neglecting vapor transport within the melt may result in underpredicting heat transfer from the pool by orders of magnitude. 
The following areas have been identified as having the potential to significantly impact heat transfer from a molten pool although additional analyses and/or data are required before any model modifications could be recommended.

- Prototypic material property effects. The limited number of experiments performed using prototypic material properties suggest that heat transfer from a molten ceramic pool may behave differently than indicated from experiments using aqueous solutions. First, experiments using $\mathrm{UO}_{2}$ (and experiments using paraffin) have observed that the upward heat flux will be suppressed because of void formation that occurs as the material undergoes a volume reduction as it solidifies. Secondly, an enhanced downward heat transfer was observed in the molten $\mathrm{UO}_{2}$ tests that cannot currently be explained. Although it has been suggested that this enhanced downward heat transfer may be due to an errors in estimating the heatup of the debris or neglecting radiation heat transfer within the debris, additional data are needed to quantitatively estimate what, if any, material property effects may impact convective heat transfer from a molten pool. Note that some of the RASPLAV tests currently planned by the Russian Research Institute will use prototypic materials and may provide insight into this area.

Solidification and melting effects on fluid motion. Although no data were found that considered the effects of solidification and melting in a volumetrically heated liquid, the data for fluids without heating indicate several effects of phase change that are not considered in SCDAP/RELAP5 molten pool heat transfer models. For example the increase in volume associated with melting has been observed to induce motion in the fluid. Furthermore, SCDAP/RELAP5 models do not consider the potential for solids to become entrained in the melt and the effect of these entrained solids on heat transfer from the pool. Finally, SCDAP/RELAP5 models do not consider the potential for voids to form within the crust as the melt solidifies. It is suggested that insights could be gained in many of these phenomena by performing additional numerical studies.

The effects of multiple-component or layer pools. The limited data available for convection in multiple component fluids suggest that heat transfer at the boundary of the heated layer is independent of the presence of an overlying non-heated layer. Furthermore, the data available for a non-heated layer above a heated layer indicate that upward heat transfer from the two layers is reduced because of the presence of the non-heated layer. No data were available for studying heat transfer from multiple layers with the heating in both layers or the top layer. Data were also not available to study the effects of crust formation or material interactions at the interface between the layers. Although the data for predicting heat transfer from multiple component pools are insufficient to recommend any SCDAP/ RELAP5 model modifications, scoping calculations discussed in Section A7.2 indicate that the current manner in which SCDAP/RELAP5 molten pool heat transfer models treat multiple component pools is unsubstantiated and it is recommended that calculations be performed to substantiate these predictions using more detailed codes. Furthermore, data from two component, two fluid layer tests that are planned in a natural convection heat transfer tests program being performed at the University of California - Santa Barbara ${ }^{\mathrm{A}-20}$ may provide some insight into these issues. 
It should be emphasized that there is still considerable uncertainty associated with many of the proposed model modifications, such as those for predicting vapor transport. Hence, it is suggested that additional analyses and/or experimental data be obtained to support these recommendations and to resolve questions identified in areas where there were insufficient data to recommend model modifications.

\section{A9. REFERENCES}

A-1. J. L. Rempe, et al., Light Water Reactor Lower Head Failure Analysis, NUREG/CR-5642, EGG-2618, October 1993.

A-2. C. M. Allison, et al., SCDAP/RELAP5/MOD3.1 Code Manual, Volume IV: MATPRO - A Library of Materials Properties for Light-Water-Reactor Accident Analysis, NUREG/CR-6150, EGG-2720, November 1993.

A-3. C. M. Allison, et al., SCDAP/RELAP5/MOD3.1 Code Manual, Volume V: Developmental Assessment, NUREG/CR-6150, EGG-2720, Volume 5, December 1993.

A-4. K. M. Kelkar, R. C. Schmidt, and S. V. Patankar, "Numerical Analysis of Laminar Natural Convection for an Internally Heated Fluid in a Hemispherical Cavity," ANS Proceedings of the 1992 National Heat Transfer Conference, Volume 6, pp. 355-364, San Diego, CA, August 1992.

A-5. F. A. Kulacki, Review and Evaluation of 'Examination of Thermohydraulic Processes and Heat Transfer in a Core Melt,' by Mayinger, Jahn, Reineke, and Steinbrenner, Letter report prepared for the U.S. NRC under contract AT(49-24)-0149, March 31, 1976.

A-6. "Compendium of Post Accident Heat Removal Models for Liquid Metal Cooled Fast Breeder Reactors," Edited by B. D. Turland and J. Morgan, European Applied Research Reports, Vol. 6, No. 5, April 1985.

A-7. F. A. Kulacki and D. E. Richards, "Natural Convection in Plane Layers and Cavities with Volumetric Energy Sources," Natural Convection, Fundamentals and Applications, Edited by S. Kakaç, W. Aung, and R. Viskanta, Hemisphere Publishing corporation, Washington, D. C., 1985.

A-8. U. Müller and T. Schulenberg, Post-Accident Heat Removal Research: A State of the Art Review, KfK 3601, November, 1983.

A-9. F. Mayinger, M. Jahn, H. H. Reineke, and V. Steinbrenner, Examination of Thermohydraulic Processes and Heat Transfer in a Core Melt, BMFT RS 48/1, Institut für Verfahrenstecknik der T. U., Hanover FRG (in German), 1976.

A-10. M. Jahn and H. H. Reineke, "Free Convection Heat Transfer with Internal Heat Sources," 5th Int. Heat Transfer Conference, Paper NC2.8, Tokyo, Japan, 1974.

A-11. U. Steinberner and H. H. Reineke, "Turbulent Buoyancy Convection Heat Transfer with Internal Heat Sources," Proc. 6th Int. Heat Transfer Conf., Vol. 2, pp. 305-310, Paper NC-21, Toronto, Canada, August 1978.

A-12. F. A. Kulacki and R. J. Goldstein, "Thermal Convection in a Horizontal Fluid Layer with Uniform Volumetric Energy Source," Journal of Fluid Mechanics, Vol. 55, Part 2, pp. 271-287, 1972.

A-13. F. A. Kulacki and A. A. Emara, "Steady and Transient Thermal Convection in a Fluid Layer with Uniform Volumetric Energy Sources," Journal of Fluid Mechanics, Vol. 83, 1977, pp. 375-395.

A-14. A. A. Emara and F. A. Kulacki, "A Numerical Investigation of Thermal Convection in a Heat -Generating Fluid Layer," Journal of Heat Transfer, Vol. 102, August 1980. 
A-15. J.H. Min and F. A. Kulacki, Steady and Transient Natural Convection with Volumetric Energy Sources in a Fluid Layer Bounded from Below by a Segment of a Sphere - Annual Report 6, July 1976- September 1977, NUREG/CR-0006, February, 1978.

A-16. O. Kymäläinen, H. Tuomisto, O. Hongisto, and T. G. Theofanous, "Heat Flux Distribution from a Volumetrically Heated Pool with High Rayleigh Number," NURETH-6 Meeting, Grenoble, France, October 1993.

A-17. J. D. Gabor, J. C. Cassulo, and P. C. Ellison, "Heat Transfer from Internally Heated Hemispherical Pools," ASME Paper 80-HAT-16, Presented at the 19th National Heat Transfer Conference, Orlando, FL, July 1980.

A-18. B. Frantz and V. Dhir, "Experimental Investigation of Natural Convection in Spherical Segments of Volumetrically Heated Pools" ASME Proceedings of the 1992 National Heat Transfer Conference, HTD-Vol. 192, Thermal Hydraulics of Severe Accidents, San Diego, CA, August 1992.

A-19. F. J. Asfia and V. K. Dhir, "Natural Convection Heat Transfer in Volumetrically Heated Spherical Pools," CSNI Workshop on Large Molten Pool Heat Transfer, Grenoble, France, March 1994.

A-20. T. Theofanous, University of California - Santa Barbara, Santa Barbara, CA, personal conversation with J. L. Rempe, Idaho National Engineering Laboratory, Idaho Falls, ID, October 5, 1994.

A-21. L. Baker Jr., M. G. Chasanov, J. D. Gabor, W. H. Gunther, J. C. Cassulo, J. D. Bingle, and G. A. Mansoori, "Heat Removal from Molten Pools," Proc. of the ANS-ENS International Meeting on Fast Reactor Safety and Related Physics, Chicago, IL, USERDA Report CONF-761001 (1976).

A-22. M. G. Chasanov, W. H. Gunther, and L. Baker, Jr., "The Production of Molten $\mathrm{UO}_{2} \mathrm{Pools}$ by Internal Heating: Apparatus and Preliminary Experimental Heat Transfer Results," Nuclear Technology, Vol. 36, pp. 120-128 (1977).

A-23. R. P. Stein, L. Baker, Jr., W. H. Gunther, and C. Cook, "Heat Transfer from Internally-Heated Molten $\mathrm{UO}_{2}$ Pools, Proc. Fourth Post Accident Heat Removal Information Exchange, pp. 1468-1472 (1979).

A-24. E. E. Anderson, "Radiative Heat Transfer in Molten $\mathrm{UO}_{2}$ based on the Rosseland Diffusion Method," Nuclear Technology, Vol. 30, p 65 (1976).

A-25. H. Vossebrecker, Interpretation of Experiments with Joule Heated Molten $\mathrm{UO}_{2}$ Pools, Interatom report, INTAT 69.00120.7 (1980).

A-26. J. Royen, "The RASPLAV Programme," Second CORVIS Task Force Meeting, Paul Scherrer Institut (PSI), Villigen, Switzerland, July 8-9, 1993.

A-27. R. K. Cole, Jr. D. P. Kelly, and M. A. Ellis, CORCON-MOD2: A Computer Program for Analysis of Molten-Core Concrete Interactions, NUREG/CR-3920, SAND84-1246, August 1984.

A-28. J. D. Gabor, L. Baker, Jr., J. C. Cassulo, and G. A. Mansoori, "Heat Transfer from Heat-Generating Boiling Pools," AIChE Symposium Series, No., 164, Vol. 73, p. 73-85, 1977.

A-29. W. R. Gustavson, J. C. Chen, and M. S. Kazimi, Heat transfer and fluid dynamic Characteristics of Internally Heated Boiling Pools, BNL-NUREG-50722, September 1977.

A-30. W. R. Gustavson, M. S. Kazimi, and J. C. Chen, "Heat Transfer and Fluid Dynamics in a Volume-Heated Boiling Pool," Proceedings of the ANS-ENS International Meeting on Fast Reactor Safety and Related Physics, Chicago, USERDA report CONF-761001, pp. 2066-2076. 
A-31. M. S. Kazimi, "The Potential for Fuel Boiling under Decay Heat Conditions," Proceedings of the Third Post Accident Heat Removal Information Exchange Meeting, Argonne, IL, ANL-78-10, pp. 377-386, 1977.

A-32. H. Gertsman and P. Griffith, "Laminar Film Condensation on the Underside of Horizontal and Inclined Surfaces," International Journal of Heat and Mass Transfer, 10, 567-580, 1967.

A-33. D. Cho and M. Epstein, Development of Governing Phenomena for Hypothetical Whole-Core Accidents, ANL-RDP-30, 7.2, 1974.

A-34. G. A. Greene, O. C. Jones Jr., C. E. Schwartz, and N. Abuaf, Heat Removal Characteristics of Volume Heated Boiling Pools with Inclined Boundaries, NUREG/CR-1357, BNL-NUREG-51157, 1980.

A-35. G. A. Greene and C. E. Schwartz, Boiling Pools with Internal Heat Generation, NUREG-CR-1249, pp. 90-96, January 1980.

A-36. T. Ginsberg and G. A. Greene, "BNL Program in Support of LWR Degraded Core Accident Analysis," Proceedings of the U. S. Nuclear Regulatory Commission Tenth Water Reactor Safety Research Information Meeting, NUREG/CP-0041, Vol. 2, pp 364-395, 1983.

A-37. Werle, H. Modellexperimente zum Kernschmelaen, Halbjahresbericht 1987/1 PNS 4332, Kerforschungszentrum, Karlsruhe, FRG, 1978.

A-38. Werle, H. Enhancement of Heat Transfer between two Horizontal Liquid Layers by Gas Injection at the Bottom, KFK 3223, Karlsruhe, FRG, 1981.

A-39. B. D. Turland and J. Morgan, "Thermal Attack of Core Debris on a PWR Reactor Vessel," Proceedings of the International Meeting on Light Water Reactor Severe Accident Evaluation, August 28-September 1, 1983, Cambridge, MA.

A-40. B. D. Turland and J. Morgan, Progression of a PWR Severe Accident from Core Melt to Cavity Interactions, EPRI-NP-4455.

A-41. American Society of Mechanical Engineers, ASME Steam Tables, 1967.

A-42. R. S. Peckover, B. D. Turland, and R. T. P. Whipple, "On the growth of melting pools in Sacrificial Materials," Proceedings of the 3rd Post Accident Heat Removal Information Exchange, Argonne, IL, 1977 (ANL-78-10).

A-43. J. R. Wolf and J. L. Rempe, TMI-2 Vessel Investigation Project Integration Report, OECD-NEA-TMI-2 Vessel Investigation Project Report TMI V(93)EG10, October 1993.

A-44. R. Viskanta, "Natural Convection in Melting and Solidification, Natural Convection, Fundamentals and Applications, Edited by S. Kakaç, W. Aung, and R. Viskanta, Hemisphere Publishing corporation, Washington, D. C., 1985.

A-45. R. D. White, A. G. Bahelt, W. Leidenfrost, and R. Viskanta, "Study of Heat Transfer and Melting from a Cylinder Imbedded in a Phase Change Material," ASME Paper No. 77-HT-42, 1977.

A-46. A. G. Bathelt and R. Viskanta, "Heat Transfer at the Solid-Liquid Interface during Melting from a Horizontal Cylinder," International Journal of Heat and Mass Transfer, Vol. 23, pp. 493-1503, 1980.

A-47. A. G. Bathelt, R. Viskanta, and W. Leidenfrost, "Latent Heat-of-Fusion Energy Storage: Experiments on Heat Transfer from Cylinders during Melting," Journal of Heat Transfer, Vol. 101, pp. 453-458, August 1979. 
A-48. C.-J. Ho and R. Viskanta, "Heat Transfer during Melting from an Isothermal Vertical Wall," Transactions of the ASME, Vol. 106, February 1984.

A-49. E. M. Sparrow and J. A. Broadbent, "Freezing in a Vertical Tube," Journal of Heat Transfer, Vol. 105, pp. 217-225, 1983.

A-50. E. M. Sparrow and P. S. Mendes, "Natural Convection Heat Transfer Coefficients Measured in Experiments on Freezing," International Journal of Heat and Mass Transfer, Vol. 25, pp. 293-297, 1982.

A-51. R. G. Kemink and E. M. Sparrow, "Heat Transfer Coefficients for Melting about a Vertical Cylinder with or without Subcooling and for Open or Closed Containment," International Journal of Heat and Mass Transfer, Vol. 24, pp. 1699-1710, 1981.

A-52. G. Fieg, "Experimental Investigations of Heat Transfer Characteristics in Liquid Layers with Internal Heat Sources," Proceedings of the ANS-ENS International Meeting on Fast Reactor Safety and Related Physics," Chicago, USERDA Report CONF-761001-P4, pp. 2047-2055, 1976.

A-53. H. Bernard, "Les tourbilons cellulaires dans une nappe liquide," Rev. Gen. Sci. Pur. Appli., Vol. 11, p. 1261, 1900.

A-54. R. Schramm and H. H. Reineke, "Natural Convection in a Horizontal Layer of two Different Fluids with Internal Heat Sources," Proceedings of the Sixth International Heat Transfer Conference, Toronto, Canada, Vol. 2, Paper NC-20, pp. 299-304, 1976.

A-55. F. A. Kulacki, A. A. Emara, J. H. Min, and A-T. Nguyen, "Experimental Studies of Steady and Transient Natural Convection with Internal Heat Sources in Enclosed Cavities," Proceedings of the Fourth Water Reactor Safety Research Meeting, Gaithersburg, MD, 1976.

A-56. F. A. Kulacki and A-T Nguyen, Hydrodynamic Instability and Thermal Convection in a Horizontal Layer of Two Immiscible Fluids with Internal Heat Generation, NUREG/CR-2619R3, 1980. 


\section{APPENDIX B}

B-1 
B-2 


\section{COMPARISON OF STRESSES IN SIDE-WALL AND BOTTOM CRUSTS}

This simplified analysis is performed to show that crust side-wall stresses are much larger than bottom stresses, so the side-wall will always fail before the bottom crust. The bottom crust is modeled as a flat plate supported by columns equidistant in the $\mathrm{x}$ and $\mathrm{y}$ direction. The side-wall is modeled as a long cylinder (i.e., end effects are neglected). A pressure differential of $\mathrm{P}$ is applied to the bottom and side-wall crusts. Stress concentration factor is assumed equal for both, so it is not used in calculating the side-wall stress-to-bottom stress ratio.

\section{B1. SIMPLIFIED ANALYSIS}

The maximum bending moment $(M)$ at the center of the plate is calculated as: ${ }^{\mathrm{B}-1}$

$\mathrm{M}=0.331 \mathrm{FPb}^{2}$

where

$\begin{array}{ll}\mathrm{M} & =\text { the moment at the center of the plate } \\ 0.331= & \text { tabulated factor which applies to a Poisson's ratio of } 0.2 \\ \mathrm{~F} & =\text { the correction factor }(=0.9377) \text { to account for Poisson's ratio of } 0.316 \\ \mathrm{P} & =\quad \text { the pressure differential } \\ \mathrm{b} & =\quad \text { the distance between column supports. }\end{array}$

The maximum bottom bending stress is:

$\sigma_{\operatorname{maxbottom}}=0.1862 \frac{\mathrm{Pb}^{2}}{\mathrm{t}^{2}}$

where
$\sigma_{\text {maxbottom }}=$
the maximum bottom bending stress
$\mathrm{t} \quad=$
the crust thickness for side-wall or bottom.

The maximum stress in the side-wall is approximated by:

$$
\sigma_{\text {maxside }- \text { wall }}=\frac{P R}{t}
$$


From Equations (B-2) and (B-3), the ratio of side-wall stress-to-bottom stress is:

$\frac{\sigma_{\text {maxside-wall }}}{\sigma_{\text {maxbottom }}}=\frac{R t}{b^{2}}$

Taking some typical dimensions of thickness $(t=20 \mathrm{~cm})$, cylinder radius $(R=146 \mathrm{~cm})$, and fuel pin spacing $(b=2.86 \mathrm{~cm})$, the ratio of side-wall stress-to-bottom stress is approximately 360 . If we assume that three out of four fuel pins do not support the crust, the support spacing doubles $(b=5.72 \mathrm{~cm})$ and the ratio is approximately 90 . For $\mathrm{R}=146 \mathrm{~cm}, \mathrm{~b}=5.72 \mathrm{~cm}$ and a thickness of $0.1 \mathrm{~cm}$, the ratio is approximately 20 .

If the ratio in Equation (B-4) is less than 1.0, then bottom crust stresses exceed side-wall stresses. For a typical crucible radius of $146 \mathrm{~cm}$, this does not occur until thickness is unreasonably small or the support spacing is large. Generally, the bottom crust is thicker and cooler than the side-wall. So long as the bottom crust is reasonably well-supported by the fuel pins, we would not expect it to fail before the side-wall.

\section{B2. REFERENCES}

B-1. S. Timoshenko and S. Woinowsky-Krieger, Theory of Plates and Shells, (McGraw-Hill, New York, 1959) pp. 245-249. 